$$
\text { Ф. Ф. Менде, А. С. Дубровин }
$$

\title{
АЛЬТЕРНАТИВНАЯ ИДЕОЛОГИЯ ЭЛЕКТРОДИНАМИКИ
}

Москва - 2016 
Менде Ф. Ф., Дубровин А. С.

Альтернативная идеология электродинамики. Монография. М.: Перо, 2016. - 198 с., ил. 62, библ. 78.

Уточняется роль векторного потенциала магнитного поля в уравнениях индукции. Вводится понятие векторного потенциала электрического поля. Показано, что кинетическая индуктивность зарядов играет в электродинамике не менее важную роль, чем диэлектрическая и магнитная проницаемость. Разработана математическая модель дисперсии электромагнитных волн в проводниках и диэлектриках с использованием физической величины диэлектрической проницаемости, которая не зависит от частоты. Показано, что в ограниченной плазме может существовать поперечный плазменный резонанс. Вводится понятие кинетической ёмкости. Приведен новый способ вывода волнового уравнения. Путём записи уравнений индукции с использованием субстанциональной производной получена их симметричная форма. Вводится скалярно-векторный потенциал, в котором скалярный потенциал заряда и его поля зависят от скорости. Из симметричных законов индукции в рамках преобразований Галилея получены преобразования полей при переходе из одной инерциальной системы отсчёта в другую, названные преобразованиями Менде. Эти преобразования позволили объяснить фазовую аберрацию и поперечный эффект Доплера, а также силовое взаимодействие токонесущих систем без использования постулата о силе Лоренца. Рассмотрены основы транскоординатной электродинамики в пространственно-временном гиперконтинууме, в которой совершенствуется аппарат дифференциального исчисления полевых функций и вводится новый оператор Дубровина. Приведены результаты экспериментальных исследований, показавших, что заряд не является инвариантом скорости. Получен взамен закона сохранения 4-импульса новый закон сохранения кинетического баланса.

Для специалистов в области электродинамики, радиофизики, электроники, радиотехники, технической защиты информации, теоретической и математической физики, а также студентов и аспирантов соответствующих специальностей.

Рецензенты

А. А. Рухадзе - доктор физико-математических наук, профессор, лауреат государственных премий и премии им. М. В. Ломоносова.

Н. Н. Горобец - доктор физико-математических наук, профессор, заведующий кафедрой прикладной электродинамики Харьковского национального университета им. В. Н. Каразина.

\section{ISBN}




\section{ВВЕДЕНИЕ}

Прошлое столетие ознаменовано величайшим кризисом в физике, когда на смену глубокому пониманию физического смысла природных явлений и технических процессов пришли новые научные ориентиры. Физик П. Дирак провозгласил математическую красоту единственным критерием для выбора пути развития в теоретической физике. Но математик М. Атья, осознавая риск быть убаюканным элегантностью, базирующейся на зыбкой почве, предупреждал, что подчинение физики математике таит в себе опасность, поскольку может завести в область измышлений, воплощающих математическое совершенство, но слишком далеких от физической реальности или даже не имеющих с ней ничего общего.

Особенностью современной физики является её сравнительно высокое финансирование при том, что осуществление прозрачного и эффективного государственного и общественного контроля за соответствующими финансовыми потоками наталкивается на значительные трудности. Ситуация, когда учёные-физики контролируют сами себя, создаёт благоприятную почву для всевозможных злоупотреблений гипертрофированными полномочиями. Особенно сложное положение дел имеет место в сфере фундаментальных физических исследований, к которой относятся, в частности, теоретические основы электродинамики. Чрезвычайно высокий уровень математизации научных работ в этой сфере приводит к тому, что даже высококвалифицированные специалисты смежных областей или всего лишь принадлежащие разным научным школам начинают говорить «на разных языках» и перестают понимать друг друга.

Научные результаты отдельных учёных (таких, как Эйнштейн и Хокинг) провозглашаются непреложной истиной подобно религиозным догматам. Но гласные и негласные запреты на критический анализ трудов признанных корифеев всегда губительны для научного прогресса и неизбежно приводят к застою. Однако любая критика должна быть объективной и конструктивной. В основе физики всегда был и должен оставаться физический эксперимент, и соответствие физическому эксперименту должно всегда быть главным критерием истинности физических теорий. Математическая строгость физических теорий также важна, но не менее важно и то, чтобы физический смысл явлений и процессов не был скрыт, завуалирован математическими формализациями. В этой связи, представляется плодотворным заимствование в теоретической электродинамике методологических подходов из электротехники (в частности, метод эквивалентных схем) ввиду того, что они позволяют разрабатывать математические модели со сравнительно простым, прозрачным физическим смыслом. Такой «электротехнический» подход к электродинамике успешно реализован в ряде работ Менде Ф.Ф. Именно опора на физический эксперимент и всестороннее раскрытие физического смысла моделей положены в основу излагаемой в данной работе альтернативной идеологии электродинамики. 
Наконец, еще один тормоз развития науки - её чрезмерная популяризация в коммерческих интересах. Наука, закованная в кандалы желтой прессы, когда на обложках популярных журналов для большего психологического эффекта изображаются якобы гениальные лица с ограниченными физическими возможностями, тенденциозно восхваляемые журналистами, абсолютно не разбирающимися в самой по себе науке, вызывает недоумение. Высокая математизация физических теорий лишь помогает желтой прессе придать физике ореол мистичности, уводя читателя от истины. Предпочтительна квалифицированная популяризация физики самими учеными, но опять-таки, она должна опираться на объективное информирование о результатах физических экспериментов и всестороннее раскрытие физического смысла теоретических моделей.

Все это и породило жесточайший кризис в современной физике. Но такое положение дел не может продолжаться вечно. Сейчас ситуация в физике очень напоминает период, предшествовавший падению системы Птолемея. На смену обветшалым догмам готовятся прийти новые прогрессивные идеи и взгляды. Чтобы лучше понять, в каком обновлении нуждается современная физика, нужно критически проанализировать, как и почему возник такой глубокий и продолжительный кризис.

Переломным моментом явился переход от сравнительно простых и интуитивно понятных классических представлений о пространстве и времени к релятивистским. А после соединения релятивизма с квантованием действия, полной геометризации гравитации и распространения принципа геометризации на другие физические взаимодействия несовершенство сложившихся идей и взглядов стало очевидным. Корень этого несовершенства заключается в принципиальном рассогласовании между физикой и математикой, когда математический аппарат физики все больше вырождается в полумистическую схоластику, в рамках которой объективный физический смысл явлений все больше ускользает, а роль субъективного сознания наблюдателя и неведомой «магической» силы абстрактных математических формул становится все более фундаментальной. Следует признать, что отмеченное рассогласование физики и математики стало нарастать задолго до победного шествия теории относительности. Наверное, исторически первым особенно «твердым орешком» для математической физики оказалась электродинамика. Переход от механики материальных точек и их конечных совокупностей к формальному описанию непрерывного в пространстве и времени электромагнитного поля требовал привлечения более мощного математического аппарата, но развитие математики, идущее, во-многом, по своим собственным внутренним законам, хронически не отвечало запросам бурно развивающейся физики.

В первоначальном виде система уравнений классической электродинамики была записана Максвеллом в его знаменитом трактате [1] с использованием исчисления кватернионов в рамках классических представлений о пространстве и времени, допускающих преобразования Галилея при переходе от рассмотрения электромагнитного поля в одной инерциальной системе отсчета (ИСО) к рассмотрению этого же поля в другой ИСО. Однако 
сразу выяснилось, что исчисление кватернионов в математике развито не настолько хорошо, чтобы физики могли его успешно применять к широкому кругу задач электродинамики. Для того чтобы привлечь в электродинамику более простые и эффективные средства математической физики, Герц и Хевисайд переформулировали уравнения Максвелла с языка исчисления кватернионов на язык векторного анализа.

В то время казалось, что формулировка Герца-Хевисайда эквивалентна исходной формулировке Максвелла, но теперь уже можно констатировать, что уравнения, полученные Герцем и Хевисайдом, являются существенным упрощением уравнений Максвелла в кватернионах, причем это упрощение относится не только к их математической форме, но и (что самое главное!) к их физическому содержанию, так как при этом уравнения лишились естественно присущей им Галилей-инвариантности. Все же для конкретно взятой ИСО (а не их совокупности) эквивалентность формулировок имела место, в силу чего формулировка Герца-Хевисайда получила заслуженное признание научного сообщества и вытеснила в теоретических и прикладных исследованиях формулировку самого Максвелла.

Анализ уравнений Герца-Хевисайда многими учеными привел к разработке Лоренцем и Пуанкаре основ математического аппарата специальной теории относительности (СТО). Это был важный шаг вперед по сравнению с нерелятивистской теорией электромагнитного поля, так как удалось выявить зависимость электромагнитного поля от относительной скорости наблюдателя. Но четкой физической интерпретации своих формул ведущие физики и математики своего времени предложить не смогли. Вот что пишет по этому поводу известный специалист в области тензорного анализа Рашевский [2]: «Теория относительности возникла в результате длительного накопления опытного материала, приведшего к глубокому преобразованию наших физических представлений о формах материи и движения. После целого ряда попыток приспособить прежние представления о пространстве, времени и других физических величинах к вновь открытым опытным фактам обнаружилось, что для этих целей требуется перестроить все эти понятия коренным образом. Эта задача была выполнена в основном А. Эйнштейном в 1905 г. (специальная теория относительности) и в 1915 г. (общая теория относительности). Впрочем, задача была выполнена лишь в том смысле, что было дано стройное формально-математическое описание нового положения вещей. Задача глубокого, подлинно физического обоснования этой математической схемы всё ещё стоит перед физикой».

В этот судьбоносный момент физика оказалась на распутьи. Один из путей лежал в направлении дальнейших поисков подходящего математического аппарата электродинамики (к чему, судя по всему, склонялись сами Лоренц и Пуанкаре), но физика вслед за Эйнштейном пошла по другому пути - решительного и бескомпромиссного отказа от классических представлений о пространстве и времени с переходом к релятивистским представлениям. Впоследствии Логунов А.А. убедительно показал, что суть СТО состоит в отождествлении естественной геометрии электромагнитно- 
го поля, описываемого уравнениями Максвелла в формулировке ГерцаХевисайда, с геометрией мирового физического пространства-времени.

Путём внедрения в физику известных постулатов теория относительности в версии Эйнштейна объяснила несколько важных экспериментальных результатов и в связи с этим получила признание широких кругов ученыхфизиков. Релятивистскую идеологию поддержали такие ведущие математики того времени, как Минковский, Гильберт и Борн. Особенно привлекателен для математиков в этой идеологии принцип геометризации, отражающий сокровенные мечты и чаяния многих мыслителей, начиная с идеалистических воззрений великого древнегреческого философа Платона, свести все фундаментальные законы Мироздания к геометрическим свойствам неких идеализированных математических объектов.

Таким образом, математика, поддавшись искушению подчинить себе физику посредством столь вожделенного принципа геометризации, оказалась не в состоянии подняться выше геометрического образа мышления и достойно удовлетворять возрастающие потребности физики. Физика использовала аппарат, который была готова предложить ей математика, что неизбежно вело к созданию специальной, а потом общей теории относительности и, далее, к все возрастающему торжеству принципа геометризации.

В соответствии с релятивистскими представлениями, зависимость электромагнитного поля от скорости движения наблюдателя не обусловлена фундаментальными факторами физической природы самого поля, а является опосредованной через зависимость от нее интервалов времени и пространственных расстояний (преобразования Лоренца) в предположении релятивистской инвариантности электрического заряда. Однако специалистами (прежде всего, экспериментаторами) было обнаружено, что классическая электродинамика и СТО, вопреки уже более столетнему мифу, находятся в контрадикции друг к другу. Современные же опыты по измерению скорости света в одном направлении (а не усредненной скорости «туда и обратно», как, например, в экспериментах Физо и им аналогичных) [3] противоречат постулату СТО о постоянстве скорости света и ставят под сомнение физическую обоснованность преобразований Лоренца.

Если все же придерживаться точки зрения фундаментальной непосредственной зависимости поля от скорости, то такая зависимость должна распространяться даже на абсолютную величину электрического заряда. До последнего времени такая не инвариантность заряда подтверждалась лишь косвенными эмпирическими данными, заключавшимися в появлении электрического потенциала на сверхпроводящих обмотках и торах при введении в них постоянного тока, а также в наблюдении электрического импульса ядерных взрывов. Однако 2015 год ознаменовался уже прямым экспериментальным подтверждением этого феномена в результате обнаружения и исследования импульса электрического поля, возникающего при разогреве плазмы в результате разряда через разрядники конденсаторов большой ёмкости. Оказалось, что в процессе разогрева плазмы при равном количестве в ней электронов и положительных ионов в ней образуется унитарный отрицательный заряд свободных электронов, не скомпенсиро- 
ванный более медленными положительными ионами. Этот факт противоречит не только классическим, но и релятивистским преобразованиям электромагнитного поля при переходе от одной ИСО к другой, свидетельствуя о несовершенстве не только классических, но и релятивистских представлений о пространстве и времени.

Идея о том, что перспективная электродинамика должна предполагать существование зависимости электромагнитного поля от скорости движения наблюдателя, обусловленной не геометрией пространства-времени, а физической природой поля, не предполагающей инвариантность электрического заряда, развивалась в ряде работ Менде Ф.Ф. В этих работах приведено обоснование введения в электродинамику вместо классических и релятивистских новых преобразований электромагнитного поля, получивших название преобразований Менде.

Однако последовательное развитие такой радикальной идеи, как не инвариантность заряда, требует глубокого пересмотра математического аппарата электродинамики, призванного к созданию математических средств более адекватного описания перехода от одной ИСО к другой. Такой подход к развитию математического аппарата электродинамики был предложен Дубровиным А.С. Этот подход лежит в рамках очередного пересмотра представлений о пространстве и времени с отказом от релятивистских и переходом к новым представлениям, которые мы называем гиперконтинуальными и считаем своего рода неоклассическими в том смысле, что они возвращают фундаментальную роль в физике преобразованиям Галилея. В то же время они допускают широкие возможности инвариантности тех или иных физических процессов относительно тех или иных групп псевдоортогональных преобразований координат, реализующих гиперболические повороты с инвариантностью псевдоевклидовой метрики эффективного (подходящим образом воображаемого) пространства-времени. Но естественная геометрия физического поля не отождествляется при этом с геометрией мирового физического пространства-времени.

Понятие пространственно-временного гиперконтинуума введено в результате совместного изучения алгебраической и геометрической структур коммутативных алгебр с единицей, элементами которых являются функции синусоидальных волн. Гипотеза гиперконтинуума (об иерархической гиперконтинуальной структуре мирового физического пространствавремени) служит отправной точкой научных исследований в направлении обобщения представлений о структуре пространства и времени в русле перехода от современной квантовой научной парадигмы к новой системной, одновременно конструктивно соединяющей в своих рамках непрерывность и дискретность, динамичность и статичность, а также глобальность и локальность. Иерархичность гиперконтинуума ограничивает применимость общепринятого принципа геометризации в физике и связанных с ним идей симметрии в геометрии за счет введения в физику идей иерархичности, эффективность которых апробирована нами при создании эталонной модели защищенной автоматизированной системы (ЭМЗАС) и математического аппарата ЭМЗАС-сетей. 
Так чего же не хватает в аппарате современной математической физики? Мы считаем, что современный математический анализ полевых функций оперирует дифференциальным исчислением с недопустимо ограниченными возможностями. Производные полевых функций и дифференциальные уравнения поля всегда относятся к конкретной системе отсчета. При переходе от одной системы отсчета к другой производятся соответствующие преобразования дифференциальных уравнений. Если они сохраняют свою форму, то говорят об их инвариантности. Но сложившийся к настоящему времени аппарат дифференциального исчисления полевых функций не предполагает рассмотрения дифференциальных уравнений поля, связывающих между собой две различные системы отсчета. Именно это не позволяет адекватно описывать переход от одной системы отсчета к другой при рассмотрении электромагнитного поля. И именно это делает актуальным разработку нового дифференциального исчисления, которое мы называем транскоординатным. Такой подход требует переформулировки уравнений электродинамики на новое представление по сравнению с представлением Герца-Хевисайда, которое мы тоже называем транскоординатным (подробнее см. в § 17). Транскоординатные дифференциальное исчисление и формулировка электродинамики разрабатывались Дубровиным А.С.

Традиционно развитие математического анализа и математической физики находилось под влиянием геометрии и связанного с ней особого геометрического образа мыслей, что и привело, в конечном итоге, к победному шествию принципа геометризации в физике. Сейчас же ситуация меняется кардинально. На развитие математики все больше влияет информатика, развивающаяся самым бурным, стремительным образом на волне революционной компьютеризации и информатизации общества. Современная информатика использует весьма высокий уровень абстракции в представлении информации, по сравнению с которым геометрический образ мыслей оказывается примитивным. Развитие гиперконтинуальных представлений о пространстве и времени вместе с идеями транскоординатности лежат в русле именно такого направления развития физико-математических наук под влиянием идей, заимствованных из информатики.

В данной монографии показано, что вслед за отказом от формулировки Герца-Хевисайда уравнений Максвелла нужно вообще отказаться от уравнений Максвелла, как таковых, вместе с лежащей в их основе концепцией электромагнитного поля. Показательна цитата из [3]: «в чем же заключается основная исходная причина противоречивости построенной Максвеллом электродинамики? Для однозначного ответа на этот вопрос... следует отметить, что еще в свое время Ампер, Гроссман, Гаусс, Ленц, Нейман, Вебер, Риман и др. стояли на точке зрения, что, не обращаясь к понятию «магнитного поля», любые магнитные взаимодействия можно свести к обычным взаимодействиям токовых элементов или движущихся зарядов... в электродинамике возобладала тогда точка зрения Фарадея и Максвелла, что электрические и «магнитные» поля являются самостоятельными физическими сущностями, хотя и связанными между собой. В сложившейся тогда исторической обстановке данные, ошибочные с физической точки зре- 
ния, допущения предопределили собой весь дальнейший ход развития электродинамики с заведомо заложенными в нее неразрешимыми противоречиями и парадоксами». И далее там же: «для непротиворечивого отражения физической сущности законов электромагнетизма необходимо полностью отказаться от любых понятий «магнитного поля» как некой самостоятельной физической сущности... для определения сил взаимодействия движущихся в физическом вакууме реального пространства электрических зарядов вполне достаточно учесть деформацию электрических полей этих зарядов, обусловленную тривиальными эффектами запаздывающих потенциалов... Остается только удивляться прозорливости Ампера, который предупреждал, что если в электродинамике не отказаться от понятия «магнит», то в дальнейшем это грозит неимоверной путаницей в теории».

В монографии приведены результаты экспериментов, подтверждающих концепцию зависимости электрического заряда от скорости, и представлен обширный материал, касающийся важнейших вопросов классической электродинамики, в частности: преобразования электромагнитного поля при переходе от одной ИСО к другой, явление фазовой аберрации, поперечный эффект Доплера, силовое взаимодействие токонесущих систем, дипольное излучение. При этом не использовались не только постулаты теории относительности, но и постулат о силе Лоренца.

Зависимость электрического заряда от скорости не нужно вводить в качестве очередного физического постулата, так как она оказывается прямым следствием предложенного Дубровиным А.С. нового динамического закона сохранения - закона сохранения кинетического баланса. Подобно законам сохранения энергии, импульса и момента импульса, он следует из вариационного принципа наименьшего действия в предположении общих свойств пространства и времени (данный закон отвечает свойству изотропии гиперконтинуума), заменяя закон сохранения 4-импульса теории относительности, отражающий свойство Лоренц-инвариантности метрики пространственно-временного континуума. Но роль данного закона далеко не ограничивается только этим. Замена закона сохранения 4-импульса на закон сохранения кинетического баланса требует радикальной перестройки механики. В частности, оказывается возможным механическое движение со сверхсветовой скоростью, раскрывается существенная специфика вращательного движения. Подобно тому, как релятивистская идеология электродинамики привела к построению релятивистской физики, предлагаемая в монографии альтернативная идеология электродинамики претендует на роль инициатора процесса создания новой, гиперконтинуальной физики. 


\section{ЧАСТЬ І. УТОЧНЕНИЕ ПОНЯТИЙ КЛАССИЧЕСКОЙ ЭЛЕКТРОДИНАМИКИ}

\section{ГЛАВА 1}

\section{ЗАКОНЫ ИНДУКЦИИ И ИХ РОЛЬ В КЛАССИЧЕСКОЙ ЭЛЕКТРОДИНАМИКЕ}

\section{§ 1. Уравнения Максвелла и сила Лоренца}

Законы классической электродинамики являются феноменологическими и отражают набор экспериментальных фактов, но их физическая природа не известна. В связи с этим современная классическая электродинамика не лишена противоречий, остающихся без своего объяснения. Для того чтобы понять эти противоречия, а также понять те цели и задачи, которые ставятся в монографии, рассмотрим существующее положение дел.

Основными уравнениями современной классической электродинамики являются уравнения Максвелла [4]. Для вакуума они имеют вид:

$$
\begin{gathered}
\operatorname{rot} \mathbf{E}=-\partial \mathbf{B} / \partial t, \\
\operatorname{rot} \mathbf{H}=\partial \mathbf{D} / \partial t, \\
\operatorname{div} \mathbf{D}=0, \\
\operatorname{div} \mathbf{B}=0,
\end{gathered}
$$

где $\mathbf{E}$ и $\mathbf{H}$ - напряженность электрического и магнитного поля, $\mathbf{D}=\varepsilon_{0} \mathbf{E}$ и $\mathbf{B}=\mu_{0} \mathbf{H}$ - электрическая и магнитная индукция, $\mu_{0}$ и $\varepsilon_{0}$ - магнитная и диэлектрическая проницаемость вакуума (магнитная и электрическая постоянная). Из (1.1)-(1.4) следуют волновые уравнения

$$
\begin{aligned}
& \nabla^{2} \mathbf{E}=\mu_{0} \varepsilon_{0} \partial^{2} \mathbf{E} / \partial t^{2}, \\
& \nabla^{2} \mathbf{H}=\mu_{0} \varepsilon_{0} \partial^{2} \mathbf{H} / \partial t^{2},
\end{aligned}
$$

которые указывают на то, что в вакууме могут распространяться плоские электромагнитные волны со скоростью света

$$
c=1 / \sqrt{\mu_{0} \varepsilon_{0}} .
$$

Для материальных сред уравнения Максвелла имеют следующий вид:

$$
\begin{gathered}
\operatorname{rot} \mathbf{E}=-\tilde{\mu} \mu_{0} \partial \mathbf{H} / \partial t=-\partial \mathbf{B} / \partial t, \\
\operatorname{rot} \mathbf{H}=n e \mathbf{v}+\tilde{\varepsilon} \varepsilon_{0} \partial \mathbf{E} / \partial t=n e \mathbf{v}+\partial \mathbf{D} / \partial t, \\
\operatorname{div} \mathbf{D}=n e, \\
\operatorname{div} \mathbf{B}=0,
\end{gathered}
$$

где $\tilde{\mu}$ и $\tilde{\varepsilon}$ - относительные магнитная и диэлектрическая проницаемость среды, а $n, e$ и $\mathbf{v}$ - плотность, величина и скорость зарядов.

Уравнения (1.1) - (1.11) записываются в заданной инерциальной системе отсчёта (ИСО), и в них отсутствуют правила перехода из одной ИСО в другую. Приведенные уравнения также предполагают, что свойства заряда не зависят от его скорости, поскольку в первом слагаемом правой части уравнения (1.9) в качестве заряда берётся его статическое значение. При- 
веденные уравнения также предполагают, что ток может течь, как в электрически нейтральной среде, где имеется равное количество зарядов обоих знаков, так и представлять обособленный поток заряженных частиц, причем обе ситуации считаются равнозначными.

В уравнениях Максвелла не содержатся указания на то, что является причиной силового взаимодействия токонесущих систем, поэтому вводится закон Ампера, из которого следует экспериментальный постулат о силе, действующей на движущийся заряд в магнитном поле. Это так называемая магнитная часть силы Лоренца [5\}

$$
\mathbf{F}_{L}=e\left[\mathbf{v} \times \mu_{0} \mathbf{H}\right] .
$$

Однако у такой аксиоматики есть существенный недостаток. Если на движущийся заряд действует сила, то в соответствии с третьим законом Ньютона должна иметь место сила реакции, уравновешивающая силу, действующую на заряд, и должно быть известно место приложения этой силы. В данном же случае магнитное поле выступает в качестве самостоятельной субстанции, являясь посредником между движущимися зарядами, и если есть необходимость найти силу их взаимодействия, то следует прибегать к услугам этого посредника. Другими словами, у нас нет закона прямого действия, который бы давал ответ на поставленный вопрос, минуя рассмотренную процедуру, т.е. мы не можем дать прямой ответ на вопрос, где находятся силы, уравновешивающие действие на заряд магнитного поля.

Соотношение (1.12) с физической точки зрение вызывает недоумение. Силы, действующие на тело в отсутствии потерь, должны быть связаны или с его ускорением, если оно осуществляет поступательное движение, или с центробежными силами, если тело осуществляет вращательное движение. Наконец, статические силы возникают в том случае, когда имеется градиент скалярного потенциала потенциального поля, в котором находится тело. Но в соотношении (1.12) ничего этого нет. Обычное прямолинейное движение вызывает силу, которая нормальна к направлению движения тела и зависит от его скорости. Это какой-то новый закон природы? На этот вопрос в классической электродинамике ответа тоже нет.

Магнитное поле является одним из важных понятий современной электродинамики. Его концепция заключается в том, что вокруг любого движущегося заряда возникает магнитное поле (закон Ампера), циркуляция которого определяется соотношением

$$
\int \mathbf{H} d \mathbf{l}=I,
$$

где $I$ - ток проводимости.

Следствием (1.13) является уравнение (1.9), если к току проводимости добавить ток смещения. Впервые ток смещения ввёл Максвелл [1].

Следует особо отметить, что введение понятия магнитного поля не имеет под собой какой-либо физической основы, а является констатацией набора экспериментальных фактов. Используя это понятие, можно при помощи определенных математических процедур получить правильный ответ при решении практических задач. Но, к сожалению, имеется ряд физических 
вопросов, при решении которых в рамках концепции магнитного поля получаются парадоксальные результаты. Вот один из них.

Из (1.12) и (1.13) следует, что при однонаправленном параллельном движении двух одноименных зарядов или потоков зарядов между ними должно возникать дополнительное притяжение. Но в ИСО, движущейся вместе с зарядами, магнитное поле отсутствует, и дополнительного притяжения нет. Этот парадокс объяснения не имеет.

Силы взаимодействия токонесущих систем приложены не только к движущимся зарядам, но и к решетке. Однако в концепции магнитного поля на этот вопрос ответа тоже нет, т.к. в (1.1)-(1.13) решетка не учитывается. В то же время, при течении тока через плазму происходит ее сжатие (так называемый пинч-эффект), при этом силы сжатия действуют не только на движущиеся электроны, но и на положительно заряженные ионы. И, опять, концепция магнитного поля не может объяснить этот факт, так как в такой концепции отсутствуют силы, которые могут действовать на ионы плазмы.

Основным законом индукции в электродинамике считается закон Фарадея, следствием которого и является первое уравнение Максвелла. Однако и здесь имеются проблемы. До сих пор считается, что униполярный генератор является исключением из правила потока.

Приведём ещё одно исключение, на которое пока никто не обратил внимание. Закон Фарадея гласит, что если через какое-то сечение изменяется магнитный поток, то в окружающем его контуре возникает вихревое электрическое поле. И если этим контуром является проводник, то в нём индуцируются токи. Таким образом, в соответствии с законом индукции Фарадея обязательным условием возникновения токов в таком контуре является изменение магнитного потока через площадку, охватываемую контуром. Если мы вносим проводящий контур в магнитное поле, то для возникновения тока, в соответствии с законом Фарадея, силовые линии магнитного поля должны пересекать сам контур. Но известно, что магнитные силовые линии не проникают в сверхпроводник и поэтому пересекать его не могут. Поэтому, если взять сверхпроводящее кольцо, то магнитный поток через его сечение всегда будет равен нулю и, пока сверхпроводник является сверхпроводником, ни при каких обстоятельствах измениться не может.

Внесём сверхпроводящее кольцо в магнитное поле. Чтобы магнитный поток через сечение кольца остался нулевым, нужно скомпенсировать внешнее магнитное поле так, чтобы магнитный поток через сечение кольца не изменился. Единственный способ - возбудить в кольце незатухающие токи, магнитные поля которых скомпенсируют внешние магнитные поля. Для возбуждения таких токов нужны электрические поля, приложенные к проводу сверхпроводящего кольца. Но как возникнут эти поля, если суммарный магнитный поток через сечение кольца не изменился, и в соответствии с законом Фарадея вихревых электрических полей быть не должно?

Все эти примеры говорят о том, что закон индукции Фарадея неточен или неполон и не отражает все возможные варианты возникновения электрических полей при изменении магнитного поля или движения в нём. 
Приведем еще одно высказывание из [4]: «Наблюдения Фарадея привели к открытию нового закона о связи электрического и магнитного полей: в области, где магнитное поле меняется со временем, генерируется электрическое поле». Но из этого закона тоже есть исключение. Вне длинного соленоида магнитные поля отсутствуют, однако при изменении тока в нём вокруг него генерируются электрические поля.

Объяснение этого факта принято относить к тому, что вокруг длинного соленоида существует циркуляция векторного потенциала [4]. Когда поток магнитной индукции внутри соленоида изменяется, возникает изменение циркуляции векторного потенциала. Эти изменения при такой интерпретации данного явления и приводят к появлению электрических полей вне соленоида. В [4] даже указывается, что в 1956 г. Бом и Аронов экспериментально обнаружили такой потенциал. Но точка зрения о существовании векторного потенциала вне длинного соленоида, где магнитные поля отсутствуют, тоже наталкивается на ряд принципиальных трудностей, которые мы обсудим при рассмотрении закона индукции Фарадея.

В классической электродинамике не находит объяснения и известное физическое явление фазовой аберрации света, когда при наблюдении звезд из движущейся ИСО, например, с Земли, телескоп необходимо наклонять на некоторый угол по направлению движения.

Из всего сказанного можно заключить, что в классической электродинамике существует ряд проблем, которые ещё ждут своего решения. Но прежде, чем перейти к решению этих проблем и наметить пути их решения, проследим тот путь, который прошла классическая электродинамика со дня её основания до наших дней.

\section{§ 2. Закон магнитоэлектрической индукции}

Главной задачей индукции является нахождение закономерностей возникновения электрических полей, а, следовательно, и сил, действующих на заряд, в данной точке пространства, т.к. только электрические поля, генерируемые тем или иным способом, оказывают силовые воздействия на заряд. Такие поля можно получить, изменяя расположение других зарядов вокруг данной точки пространства или ускоряя их. Если вокруг рассматриваемой точки имеется какая-то статическая конфигурация зарядов, то напряженность электрического поля в данной точке определяется соотношением $\mathbf{E}=-\operatorname{grad} \varphi$, где $\varphi$ скалярный потенциал в заданной точке, определяемый данной конфигурацией. Другой конфигурации зарядов соответствуют другие значения скалярного потенциала, а, следовательно, и другие значения напряженности электрического поля в заданной точке. Но перемещение зарядов в обязательном порядке сопряжено с их ускорением и последующим замедлением. Ускорение или замедление зарядов также может приводить к возникновению в окружающем пространстве индукционных электрических полей. Может возникнуть и другая стационарная ситуация, когда, например, после ускорения заряды движутся с постоянной 
скоростью в окрестностях рассматриваемой точки, например, по круговым или другим замкнутым траекториям. В этом случае также могут возникать конфигурационные электрические поля за счет наличия пространственных градиентов скоростей в потоках движущихся зарядов.

Основным законом индукции в электродинамике является закон Фарадея $[6,7]$. В современной электродинамике он записывается в виде

$$
\text { ff } \mathbf{E} d \vec{l}=-\frac{\partial \Phi_{B}}{\partial t}=-\mu \int \frac{\partial \mathbf{H}}{\partial t} d \mathbf{s}=-\int \frac{\partial \mathbf{B}}{\partial t} d \mathbf{s},
$$

где $\mathbf{B}=\mu \mathbf{H}$ - вектор магнитной индукции, $\Phi_{B}=\mu \int \mathbf{H} d \mathbf{s}-$ поток магнитной индукции, а $\mu=\tilde{\mu} \mu_{0}$ - магнитная проницаемость среды. Из этого закона следует, что циркуляция вектора электрического поля равна изменению потока магнитной индукции через площадку, которую охватывает данный контур. Сразу необходимо отметить, что рассматриваемый закон представляет процессы взаимной индукции, т.к. для получения циркуляции вектора $\mathbf{E}$ мы берем стороннее магнитное поле, сформированное сторонними источниками. Из (2.1) получают первое уравнение Максвелла

$$
\operatorname{rot} \mathbf{E}=-\partial \mathbf{B} / \partial t
$$

Сразу укажем на терминологическую ошибку. Закон Фарадея следует называть законом не электромагнитной, как это принято в существующей литературе, а магнитоэлектрической индукции, т.к. изменение магнитных полей приводит к возникновению электрических полей, а не наоборот.

Введём векторный магнитный потенциал магнитного поля $\mathbf{A}_{H}$ :

$$
\mu \llbracket \mathbf{A}_{H} d \mathbf{l}=\Phi_{B}
$$

где контур интегрирования совпадает с контуром интегрирования в соотношении (2.1), а вектор $\mathbf{A}_{H}$ определен на всех его участках, тогда

$$
\mathbf{E}=-\mu \partial \mathbf{A}_{H} / \partial t
$$

Введенный вектор $\mathbf{A}_{H}$ предполагает локальную связь между ним и электрическим полем, а также между пространственными производными этого вектора и магнитным полем. Он связан с магнитным полем:

$$
\operatorname{rot} \mathbf{A}_{H}=\mathbf{H} \text {. }
$$

Таким образом, если определён вектор $\mathbf{A}_{H}$, его локальная производная по времени в любой точке пространства, а также его пространственные производные, то можно определять сразу и вектор $\mathbf{E}$, и вектор $\mathbf{H}$.

Векторный потенциал магнитного поля можно непосредственно получить и из закона Ампера, который был известен задолго до уравнений Максвелла. Этот закон, выраженный в векторной форме, определяет магнитное поле в точке $x, y, z$ в следующем виде:

$$
\mathbf{H}=\frac{1}{4 \pi} \int \frac{I d \mathbf{l} \times \mathbf{r}}{r^{3}},
$$

где $I$ - ток в элементе $d \mathbf{l}, \mathbf{r}$ - вектор, направленный из $d \mathbf{l}$ в точку $x, y, z$.

Можно показать, что

$$
\frac{[\mathbf{r} d \mathbf{l}]}{r^{3}}=\operatorname{grad}\left(\frac{1}{r}\right) \times d \mathbf{l} ; \operatorname{grad}\left(\frac{1}{r}\right) \times d \mathbf{l}=\operatorname{rot}\left(\frac{d \mathbf{l}}{r}\right)-\frac{1}{r} \operatorname{rot} d \mathbf{l} .
$$


Но ротор $d \mathbf{l}$ равен нулю и поэтому окончательно

$$
\mathbf{H}=\operatorname{rot} \int\left(\frac{d \mathbf{l}}{4 \pi r}\right)=\operatorname{rot} \mathbf{A}_{H},
$$

где

$$
\mathbf{A}_{H}=\int I\left(\frac{d \mathbf{l}}{4 \pi r}\right)
$$

В данном случае векторный потенциал определён уже не через магнитный поток, а через ток, текущий через определённый участок проводника. Замечательным свойством этого выражения является то, что векторный потенциал зависит от расстояния до точки наблюдения, как $1 / r$. Именно это его свойство и позволяет получить законы излучения.

Поскольку $I=g v$, где $g$ - погонный заряд (количество зарядов, приходящееся на единицу длины проводника), далее получаем:

$$
\mathbf{A}_{H}=\int \frac{g v d \mathbf{l}}{4 \pi r} .
$$

Для одиночного заряда $е$ это соотношение принимает вид:

$$
\mathbf{A}_{H}=\frac{e \mathbf{v}}{4 \pi r}
$$

а поскольку выполняется соотношение (2.3), то

$$
\mathbf{E}=-\mu \int \frac{g \frac{\partial v}{\partial t} d \mathbf{l}}{4 \pi r}=-\mu \int \frac{g a d \mathbf{l}}{4 \pi r}
$$

где $a$ - ускорение зарядов.

Для одиночного заряда это соотношение выглядит следующим образом:

$$
\mathbf{E}=-\frac{\mu e \mathbf{a}}{4 \pi r} \text {. }
$$

Это и есть закон индукции, который связывает возникновение электрических полей непосредственно с ускорением заряда.

Если нужно найти индуцируемые электрические поля при движении в поле пространственно-меняющегося векторного потенциала, следует использовать полную производную:

$$
\mathbf{E}^{\prime}=-\mu d \mathbf{A}_{H} / d t
$$

Штрих около вектора $\mathbf{E}$ означает, что это поле определяется в движущейся системе координат. Это означает, что электрическое поле может иметь не только локальную, но и конвекционную составляющую, т.е. может изменяться, как за счет локального изменения времени, так и за счет движения в пространственно-меняющемся поле векторного потенциала. При этом соотношение (2.5) можно переписать следующим образом:

$$
\mathbf{E}^{\prime}=-\mu \frac{\partial \mathbf{A}_{H}}{\partial t}-\mu(\mathbf{v} \nabla) \mathbf{A}_{H},
$$

где $\mathbf{v}$ - скорость штрихованной системы.

Конвективная часть силы, действующая на заряд в движущейся системе,

$$
\mathbf{F}_{v, 1}^{\prime}=-\mu e(\mathbf{v} \nabla) \mathbf{A}_{H}
$$


зависит только от пространственных производных векторного потенциала и скорости заряда.

Заряд, движущийся в поле векторного потенциала $\mathbf{A}_{H}$ со скоростью $\mathbf{v}$ обладает потенциальной энергией [4]

$$
W=-e \mu\left(\mathbf{v} \mathbf{A}_{H}\right) \text {. }
$$

Поэтому должна существовать еще одна сила, действующая на заряд в движущейся системе координат, а именно:

$$
\mathbf{F}_{v, 2}^{\prime}=-\operatorname{grad} W=e \mu \operatorname{grad}\left(\mathbf{v} \mathbf{A}_{H}\right) .
$$

Таким образом, величина $\left(\mathbf{v} \mathbf{A}_{H}\right)$ играет такую же роль, как и скалярный потенциал $\varphi$, градиент которого также дает силу. Следовательно, суммарная сила, которая действует на заряд, движущийся в поле векторного потенциала, может иметь три составляющие и запишется как

$$
\mathbf{F}^{\prime}=-e \mu \frac{\partial \mathbf{A}_{H}}{\partial t}-e \mu(\mathbf{v} \nabla) \mathbf{A}_{H}+e \mu \operatorname{grad}\left(\mathbf{v} \mathbf{A}_{H}\right) .
$$

Первая из составляющих этой силы действует на неподвижный заряд, когда векторный потенциал имеет локальную производную по времени. Вторая составляющая связана с движением заряда в пространственноменяющемся поле этого потенциала. Совсем иная природа у силы, которая определяется последним слагаемым соотношения (2.6). Она связана с тем, что заряд, движущийся в поле векторного потенциала, обладает потенциальной энергией, градиент которой и дает силу. Из (2.6) следует

$$
\mathbf{E}^{\prime}=-\mu \frac{\partial \mathbf{A}_{H}}{\partial t}-\mu(\mathbf{v} \nabla) \mathbf{A}_{H}+\mu \operatorname{grad}\left(\mathbf{v} \mathbf{A}_{H}\right)
$$

Это и есть полный закон взаимной индукции. Он определяет все электрические поля в заданной точке пространства, причем эта точка может быть как неподвижной, так и движущейся. Этот единый закон включает в себя и закон Фарадея и ту часть силы Лоренца, которая связана с движением заряда в магнитном поле, и без всяких исключений дает ответ на все вопросы, касающиеся взаимной магнитоэлектрической индукции. Показательно, что, если взять ротор от обеих частей равенства (2.7), пытаясь получить первое уравнение Максвелла, то сразу будет потеряна существенную часть информации, т.к. ротор от градиента тождественно равен нулю.

Если выделить те силы, которые связаны с движением заряда в пространственно-меняющемся поле векторного потенциала, и учесть, что

$$
\mu \operatorname{grad}\left(\mathbf{v} \mathbf{A}_{H}\right)-\mu(\mathbf{v} \nabla) \mathbf{A}_{H}=\mu\left[\mathbf{v} \times \operatorname{rot} \mathbf{A}_{H}\right]
$$

то из (2.6) получим

$$
\mathbf{F}_{v}^{\prime}=e \mu\left[\mathbf{v} \times \operatorname{rot} \mathbf{A}_{H}\right]
$$

и, учитывая (2.4), запишем

$$
\mathbf{F}_{v}^{\prime}=e \mu[\mathbf{v} \times \mathbf{H}]
$$

Это и есть магнитная часть силы Лоренца.

Электрическое поле, действующее на заряд в этом случае, запишется как

$$
\mathbf{E}_{v}^{\prime}=\mu[\mathbf{v} \times \mathbf{H}]
$$


и окончательно:

$$
\mathbf{F}^{\prime}=e \mathbf{E}+e \mathbf{E}_{v}^{\prime}=-e \frac{\partial \mathbf{A}_{H}}{\partial t}+e \mu[\mathbf{v} \times \mathbf{H}] .
$$

Может показаться, что соотношение (2.11) представляет полную силу Лоренца, однако это не так. В этом соотношении и поле $\mathbf{E}$, и поле $\mathbf{E}_{v}^{\prime}$ являются индуцированными: первое из них связано с локальной производной векторного потенциала по времени, второе обязано движению заряда в пространственно-меняющемся поле этого потенциала. Чтобы получить полную силу, действующую на заряд, необходимо к правой части соотношения (2.11) добавить слагаемое $-e \operatorname{grad} \varphi$ :

$$
\mathbf{F}_{\Sigma}^{\prime}=-e \operatorname{grad} \varphi-e \frac{\partial \mathbf{A}_{H}}{\partial t}+e \mu[\mathbf{v} \times \mathbf{H}],
$$

где $\varphi$ - скалярный потенциал в точке наблюдения.

Теперь соотношение (2.7) можно переписать следующим образом:

$$
\mathbf{E}^{\prime}=-\mu \frac{\partial \mathbf{A}_{H}}{\partial t}-\mu(\mathbf{v} \nabla) \mathbf{A}_{H}+\mu \operatorname{grad}\left(\mathbf{v} \mathbf{A}_{H}\right)-\operatorname{grad} \varphi,
$$

или, собрав первые два члена в полную производную векторного потенциала по времени, а также, внеся под знак градиента два последних члена правой части соотношения (2.12), получим:

$$
\mathbf{E}^{\prime}=-\mu \frac{d \mathbf{A}_{H}}{d t}+\operatorname{grad}(\mu(\mathbf{v A})-\varphi) .
$$

Если обе части (2.12) умножить на величину заряда, то получится полная сила, действующая на заряд. От силы Лоренца она будет отличаться слагаемым $-e \mu \partial \mathbf{A}_{H} / \partial t$. Из (2.13) видно, что величина $\mu(\mathbf{v A})-\varphi$ играет роль обобщенного скалярного потенциала. Взяв ротор от обеих частей (2.13) и учитывая, что $\operatorname{rot} \operatorname{grad}=0$, получим:

$$
\operatorname{rot} \mathbf{E}^{\prime}=-\mu d \mathbf{H} / d t .
$$

Если в данном соотношении заменить полную производную на частную, то получим первое уравнение Максвелла. Такой подход максимально прояснил физическую картину взаимной индукции. Мы специально посмотрели на этот закон под другим углом зрения для разрешения тех противоречивых суждений, которые имеют место в классической электродинамике.

Ранее сила Лоренца рассматривалась, как фундаментальный экспериментальный постулат, не связанный с законом индукции. Расчетным путем получить последнее слагаемое правой части соотношения (2.11) можно было только в рамках СТО, введя два постулата этой теории. В данном случае все слагаемые соотношения (2.11) получены из закона индукции в рамках преобразований Галилея. Причем соотношение $(2.11)$ - это и есть полный закон взаимной индукции, если его записать в терминах векторного потенциала. И это есть как раз то правило, которое дает возможность, зная поля в одной ИСО, вычислять поля в другой. 


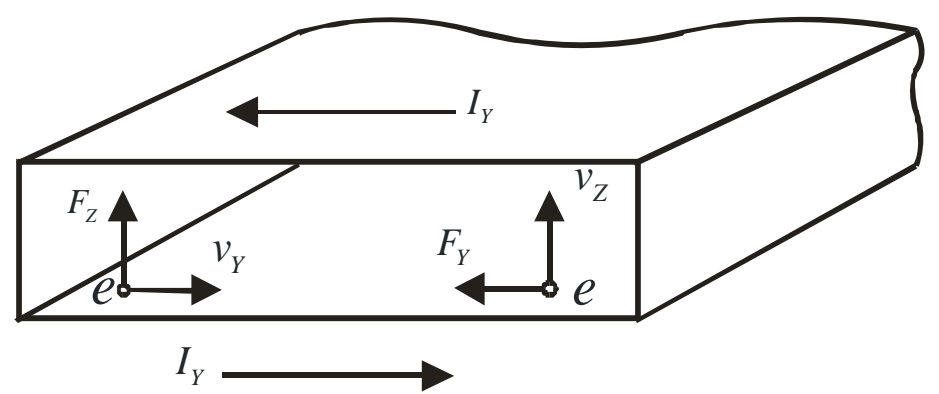

Рис. 1. Силы, действующие на заряд, движущийся в поле векторного потенциала

Структуру сил, действующих на движущийся заряд, легко понять, рассматривая движение заряда между двумя параллельными плоскостями, по которым течёт ток (рис. 1). Выберем оси координат таким образом, чтобы ось $z$ была направлена нормально к плоскостям, а ось $y$ параллельна им.

Для случая, когда расстояние между пластинами значительно меньше их размеров (в данном случае на картинке это соотношение не соблюдено), магнитное поле $H_{x}$ между ними будет равно удельному току $I_{y}$, текущему по пластинам. Если положить, что векторный потенциал на нижней пластине равен нулю, то его $y$-компонента, отсчитываемая от нижней пластины, будет возрастать по закону

$$
A_{y}=I_{y} z \text {. }
$$

Если заряд двигается в направлении оси $y$ вблизи нижней пластины со скоростью $v_{y}$, то сила $F_{z}$, действующая на заряд, определяется последним слагаемым соотношения (2.6) и равна

$$
F_{z}=e \mu v_{y} I_{y} .
$$

Эта сила направлена от нижней пластины к верхней.

Если заряд движется вдоль оси $z$ от нижней пластины к верхней со скоростью $v_{z}=v_{y}$, то для нахождения силы следует использовать уже второе слагаемое правой части (2.6). Эта сила по абсолютной величине опять равна силе (2.14), и направлена в сторону, противоположную оси $y$. При любых других направлениях движения суммарная сила будет векторной суммой двух сил, представляемых последними слагаемыми (2.6). Суммарная же величина этой силы будет определяться (2.11), а сама сила всегда будет нормальной к направлению движения заряда. Раньше рассматривалось наличие такой силы как действие силы Лоренца, природу которой была неясна, и вводилась она как экспериментальный постулат. Теперь понятно, что она является следствием совместного действия двух сил, различных по своей природе, физический смысл которых теперь ясен.

Понимание структуры сил дает возможность посмотреть на уже известные явления с другой точки зрения. Например, с чем связано существование сил, которые растягивают петлю с током? В данном случае это обстоятельство может интерпретироваться не как действие силы Лоренца, а с энергетической точки зрения. Ток, текущий по элементу кольцевого витка, 
находится в поле векторного потенциала, создаваемого остальными элементами этого витка, а, следовательно, имеет запас потенциальной энергии. Сила, действующая на такой элемент, обусловлена наличием градиента потенциальной энергии этого элемента и пропорциональна градиенту скалярного произведения величины тока на векторный потенциал в данной точке. Таким образом, можно объяснить и происхождение пондеромоторных (механических) сил. Если ток разбить на отдельные токовые нити, то все они по отдельности создают поле векторного потенциала. Суммарное поле действует на каждую нить в отдельности, и в соответствии с последним слагаемым правой части (2.6) это будет приводить к взаимному притяжению. И в первом, и во втором случае в соответствии с общими принципами система стремится к минимуму потенциальной энергии.

Следует подчеркнуть, что в (2.8) и (2.9) все поля имеют индукционное происхождение, и они связаны толи с локальной производной векторного потенциала, толи с движением заряда в пространственно-меняющемся поле этого потенциала. Если поля во времени не изменяются, то в правой части (2.8) и (2.9) остаются только последние слагаемые, и они объясняют работу всех существующих электрогенераторов с движущимися механическими частями, в том числе и работу униполярного генератора. Соотношение (2.7) дает возможность физически объяснить все составляющие напряженности электрического поля, возникающего в неподвижной и движущейся систем координат. В случае униполярного генератора в формировании силы, действующей на заряд, принимают участие два последних слагаемых правой части равенства (2.7), внося одинаковые вклады.

Сам Фарадей опытно установил, что в контуре индуцируется ток, когда в соседнем контуре включается или выключается постоянный ток или соседний контур с постоянным током движется относительно первого контура. Поэтому общий вид закона Фарадея следующий:

$$
f \mathbf{E}^{\prime} d \mathbf{l}^{\prime}=-\frac{d \Phi_{B}}{d t} .
$$

Данная запись закона указывает на то, что при определении циркуляции $\mathbf{E}$ в движущейся (штрихованной) системе координат, около $\mathbf{E}$ и $d \mathbf{l}$ должны стоять штрихи и следует брать полную производную. Если же циркуляция определяется в неподвижной системе координат, то штрихи около $\mathbf{E}$ и $d \mathbf{l}$ отсутствуют, но справа в выражении (2.15) стоит частная производная по времени. Обычно при записи закона магнитоэлектрической индукции на этом внимание в существующей литературе почему-то не акцентируется.

Полная производная по времени в (2.15) означает независимость конечного результата появления электродвижущей силы (ЭДС) в контуре от способа изменения потока: либо за счет локальной производной по времени магнитной индукции В , либо за счет того, что система, в которой измеряется циркуляция ff: $\mathbf{E}^{\prime} d \mathbf{l}^{\prime}$, двигается в пространственно-меняющемся поле В . Величина магнитного потока в (2.15) дается выражением

$$
\Phi_{B}=\int \mathbf{B} d \mathbf{s}^{\prime},
$$


где магнитная индукция $\mathbf{B}=\mu \mathbf{H}$ определена в неподвижной системе координат, а элемент $d \mathbf{s}^{\prime}$ определен в движущейся системе.

Учитывая (2.15), из (2.16) получаем

$$
\int \mathbf{E}^{\prime} d \mathbf{l}^{\prime}=-\frac{d}{d t} \int \mathbf{B} d \mathbf{s}^{\prime}
$$

и далее, поскольку $\frac{d}{d t}=\frac{\partial}{\partial t}+\mathbf{v g r a d}$, запишем:

$$
\int \mathbf{E} d \mathbf{l}^{\prime}=-\int \frac{\partial \mathbf{B}}{\partial t} d \mathbf{s}^{\prime}-\int[\mathbf{B} \times \mathbf{v}] d \mathbf{l}^{\prime}-\int \mathbf{v} \operatorname{div} \mathbf{B} d \mathbf{s}^{\prime} .
$$

В данном случае контурный интеграл берется по контуру $d l^{\prime}$, охватывающему площадку $d \mathbf{s}^{\prime}$. Сразу отметим, что все дальнейшее изложение будет вестись в предположении справедливости преобразований Галилея, т.е. $d \mathbf{l}^{\prime}=d \mathbf{l}$ и $d \mathbf{s}^{\prime}=d \mathbf{s}$. Из (2.17) следует хорошо известный результат

$$
\mathbf{E}^{\prime}=\mathbf{E}+[\mathbf{v} \times \mathbf{B}],
$$

из которого следует, что при движении в магнитном поле возникает дополнительное электрическое поле, определяемое последним слагаемым соотношения (2.18). Заметим, что это соотношение получено не путем введения постулата о силе Лоренца или с использованием преобразований Лоренца, а непосредственно из закона Фарадея, причем в рамках преобразований Галилея. Таким образом, сила Лоренца является прямым следствием закона магнитоэлектрической индукции.

Из закона Ампера следует соотношение

$$
\mathbf{H}=\operatorname{rot} \mathbf{A}_{H} .
$$

Тогда (2.17) для индуцированных полей можно переписать в виде

$$
\mathbf{E}^{\prime}=-\mu \frac{\partial \mathbf{A}_{H}}{\partial t}+\mu[\mathbf{v} \times \operatorname{rot} \mathbf{A}]
$$

и далее:

$$
\mathbf{E}^{\prime}=-\mu \frac{\partial \mathbf{A}_{H}}{\partial t}-\mu(\mathbf{v} \nabla) \mathbf{A}_{H}+\mu \operatorname{grad}\left(\mathbf{v} \mathbf{A}_{H}\right)
$$

Опять получилось соотношение (2.7), но получено оно непосредственно из закона Фарадея. Правда, и этот путь пока не проливает свет на физическую природу происхождения силы Лоренца, так как истинные физические причины возникновения векторного потенциала нам пока не ясны.

При рассмотрении сил, действующих на заряд, мы ограничились случаем, когда время запаздывания, необходимое для прохождения сигнала от источника, генерирующего векторный потенциал, до самого заряда было значительно меньше, чем период колебаний тока в проводниках. Теперь снимем такое ограничение.

Второе уравнение Максвелла в терминах векторного потенциала можно записать следующим образом:

$$
\operatorname{rot} \operatorname{rot} \mathbf{A}_{H}=\mathbf{j}\left(\mathbf{A}_{H}\right) \text {, }
$$

где $\mathbf{j}\left(\mathbf{A}_{H}\right)$ - некоторый функционал от $\mathbf{A}_{H}$, зависящий от свойств рассматриваемой среды. Если, например, $\mathbf{j}=\sigma \mathbf{E}$, т.е. выполняется закон Ома, то 


$$
\left(\mathbf{j} \mathbf{A}_{H}\right)=-\sigma \mu \frac{\partial \mathbf{A}_{H}}{\partial t} .
$$

Для свободного пространства имеет место соотношение

$$
\mathbf{j}\left(\mathbf{A}_{H}\right)=-\mu \varepsilon \frac{\partial^{2} \mathbf{A}_{H}}{\partial t^{2}} .
$$

Для свободных зарядов, движущихся без потерь, функционал имеет вид:

$$
\mathbf{j}\left(\mathbf{A}_{H}\right)=-\frac{\mu}{L_{k}} \mathbf{A}_{H}
$$

где $L_{k}=\frac{m}{n e^{2}}$ - кинетическая индуктивность зарядов [8]. В этом соотношении $m, e$ и $n$ - масса заряда, его величина и плотность соответственно.

Соотношения (2.21)-(2.23) отражают известный факт о существовании трёх видов электрического тока: активного и двух реактивных. Каждый из них имеет характерную зависимость от векторного потенциала. Эта зависимость и определяет правила распространения векторного потенциала в различных средах. Здесь следует подчеркнуть, что написание соотношений (2.21)-(2.23) предполагает не только наличие тока, но и наличие тех материальных сред, которые могут такие токи пропускать. Ток проводимости, определяемый соотношениями (2.21) и (2.23), могут пропускать через себя проводники, в которых имеются свободные носители тока. Ёмкостной ток, или ток смещения, может проходить через свободное пространство или диэлектрики. Для свободного пространства соотношение (2.20) имеет вид:

$$
\operatorname{rot} \operatorname{rot} \mathbf{A}_{H}=-\mu \varepsilon \frac{\partial^{2} \mathbf{A}_{H}}{\partial t^{2}} .
$$

Это волновое уравнение свидетельствует о том, что векторный потенциал может распространяться в свободном пространстве в виде плоских волн, и оно по своей информативности не уступает волновым уравнениям для электрических и магнитных полей, полученным из уравнений Максвелла.

Итак, запись закона индукции в терминах векторного потенциала имеет более глубокий физический смысл, чем запись этого закона в формулировке Фарадея. Такой подход проливает свет на многие физические явления, которые ранее понятны не были. Проблема заключается лишь в том, что нам пока не ясна физическая природа векторного потенциала магнитного поля. И, если удастся выяснить его физическую природу, то будет решена очень важная задача, как теоретического, так и прикладного плана.

\section{§ 3. Закон электромагнитной индукции}

Закон Фарадея показывает, каким образом изменение магнитных полей приводит к появлению электрических полей. Однако возникает вопрос о том, приводит ли изменение электрических полей к возникновению какихлибо других полей и, в частности, магнитных? Ответ на этот вопрос дал Максвелл, введя ток смещения в свое второе уравнение. В случае отсутствия токов проводимости второе уравнение Максвелла имеет вид: 


$$
\operatorname{rot} \mathbf{H}=\varepsilon \frac{\partial \mathbf{E}}{\partial t}=\frac{\partial \mathbf{D}}{\partial t},
$$

где $\mathbf{D}=\varepsilon \mathbf{E}$ - электрическая индукция.

От этого соотношения нетрудно перейти к выражению

$$
\text { f] } \mathbf{H} d \mathbf{l}=\partial \Phi_{E} / \partial t
$$

где $\Phi_{E}=\int \mathbf{D} d \mathbf{s}$ - поток электрической индукции.

Однако для полного описания процессов взаимной электрической индукции соотношения (3.1) недостаточно. Как и в случае закона Фарадея, следует учесть то обстоятельство, что поток электрической индукции может меняться не только за счет локальной производной электрического поля по времени, но и за счет того, что контур, вдоль которого производится интегрирование, может двигаться в пространственно-меняющемся электрическом поле. Это означает, что в соотношении (3.1), как и в случае закона Фарадея, следует заменить частную производную на полную. Обозначая штрихами поля и элементы контура в движущейся ИСО, получим:

$$
\text { ff } \mathbf{H}^{\prime} d \mathbf{l}^{\prime}=\frac{d \Phi_{E}}{d t}
$$

и далее

$$
\int \mathbf{H}^{\prime} d \mathbf{l}^{\prime}=\int \frac{\partial \mathbf{D}}{\partial t} d \mathbf{s}^{\prime}+f[\mathbf{D} \times \mathbf{v}] d \mathbf{l}^{\prime}+\int \mathbf{v} \operatorname{div} \mathbf{D} d \mathbf{s}^{\prime}
$$

Для электронейтральной среды $\operatorname{div} \mathbf{E}=0$, поэтому последний член правой части в (3.2) отсутствует. Для этого случая (3.2) имеет вид:

$$
\text { ff } \mathbf{H}^{\prime} d \mathbf{l}^{\prime}=\int \frac{\partial \mathbf{D}}{\partial t} d \mathbf{s}^{\prime}+f[\mathbf{D} \times \mathbf{v}] d \mathbf{l}^{\prime} .
$$

Если в этом соотношении перейти от интегрирования по контуру к интегрированию по поверхности, то получим:

$$
\operatorname{rot} \mathbf{H}^{\prime}=\frac{\partial \mathbf{D}}{\partial t}+\operatorname{rot}[\mathbf{D} \times \mathbf{v}]
$$

Если, учитывая это соотношение, записать поля в данной инерциальной системе, то штрих около Н и второй член правой части исчезнут, и получим ток смещения, введенный Максвеллом. Но Максвелл ввел этот параметр, не прибегая к закону электромагнитной индукции (3.2). Если свой закон магнитоэлектрической индукции Фарадей вывел на основании экспериментов с магнитными полями, то эксперименты по установлению справедливости соотношения (3.2) в то время провести было невозможно, т.к. для проведения такого эксперимента не хватало чувствительности существующих измерительных приборов.

Для случая постоянных электрических полей из (3.3) получаем:

$$
\mathbf{H}_{v}=-\varepsilon[\mathbf{v} \times \mathbf{E}] \text {. }
$$

Теперь электрические поля можно выразить через ротор электрического векторного потенциала, положив [8]

$$
\mathbf{E}=\operatorname{rot} \mathbf{A}_{E} \text {. }
$$

Соотношение (3.4) с учетом (3.6) запишется в виде: 


$$
\mathbf{H}^{\prime}=\varepsilon \frac{\partial \mathbf{A}_{E}}{\partial t}-\varepsilon\left[\mathbf{v} \times \operatorname{rot} \mathbf{A}_{E}\right]
$$

Далее можно повторить все те процедуры, которые уже проводились с магнитным векторным потенциалом, и записать следующие соотношения:

$$
\begin{gathered}
\mathbf{H}^{\prime}=\varepsilon \frac{\partial \mathbf{A}_{E}}{\partial t}+\varepsilon(\mathbf{v} \nabla) \mathbf{A}_{E}-\varepsilon \operatorname{grad}\left(\mathbf{v} \mathbf{A}_{E}\right), \\
\mathbf{H}^{\prime}=\varepsilon \frac{\partial \mathbf{A}_{E}}{\partial t}-\varepsilon\left[\mathbf{v} \times \operatorname{rot} \mathbf{A}_{E}\right] \\
\mathbf{H}^{\prime}=\varepsilon \frac{d A_{E}}{d t}-\varepsilon \operatorname{grad}\left(\mathbf{v} \mathbf{A}_{E}\right) .
\end{gathered}
$$

Данное рассмотрение можно было бы, как в случае закона магнитоэлектрической индукции, начать с введения вектора $\mathbf{A}_{E}$, но этот путь пройден традиционным способом, начиная с интегрального закона, чтобы показать идентичность процессов для двух различных законов и логическую последовательность введения электрического векторного потенциала.

Использование субстанциональной производной в законах индукции существенно проясняет физику этих процессов и даёт возможность выделить составляющие силы, действующие на заряд. Это даёт также возможность получить законы преобразования полей при переходе из одной ИСО в другую. В этом и состоит модернизация старой электродинамики, хотя физическая сущность введённых потенциалов остаётся, как и ранее, не ясна [9, 10].

\section{§ 4. Множественность форм записи законов электродинамики}

В предыдущем параграфе показано, что магнитные и электрические поля могут быть выражены через их векторные потенциалы

$$
\begin{aligned}
& \mathbf{H}=\operatorname{rot} \mathbf{A}_{H}, \\
& \mathbf{E}=\operatorname{rot} \mathbf{A}_{E} .
\end{aligned}
$$

В терминах этих потенциалов можно записать уравнения Максвелла:

$$
\begin{aligned}
& \operatorname{rot} \mathbf{A}_{E}=-\mu \frac{\partial \mathbf{A}_{H}}{\partial t}, \\
& \operatorname{rot} \mathbf{A}_{H}=\varepsilon \frac{\partial \mathbf{A}_{E}}{\partial t} .
\end{aligned}
$$

Для каждого из введённых потенциалов можно получить волновое уравнение, в частности

$$
\operatorname{rot} \operatorname{rot} \mathbf{A}_{E}=-\varepsilon \mu \frac{\partial^{2} \mathbf{A}_{E}}{\partial t^{2}}
$$

и считать, что в пространстве распространяются не магнитные и электрические поля, а поле электрического векторного потенциала.

При этом, как легко видеть из соотношений (4.1)-(4.4), магнитное и электрическое поле определятся через этот потенциал соотношениями:

$$
\mathbf{H}=\varepsilon \frac{\partial \mathbf{A}_{E}}{\partial t} ; \quad \mathbf{E}=\operatorname{rot} \mathbf{A}_{E}
$$


Пространственная производная $\operatorname{rot} \mathbf{A}_{E}$ и локальная производная по времени $\frac{\partial \mathbf{A}_{E}}{\partial t}$ связаны волновым уравнением (4.5).

Таким образом, использование только одного электрического векторного потенциала позволяет полностью решить задачу о распространении электрического и магнитного полей. Учитывая (4.6), теперь вектор Пойнтинга можно записать только через вектор $\mathbf{A}_{E}$ :

$$
\mathbf{P}=\varepsilon\left[\frac{\partial \mathbf{A}_{E}}{\partial t} \times \operatorname{rot} \mathbf{A}_{E}\right] .
$$

Характерно то, что при таком подходе обязательным условием распространения является наличие в данной точке пространства, как временных, так и пространственных производных одного и того же потенциала.

Данную задачу можно решить и другим способом, записав волновое уравнение для магнитного векторного потенциала:

$$
\operatorname{rot} \operatorname{rot} \mathbf{A}_{H}=-\varepsilon \mu \frac{\partial^{2} \mathbf{A}_{H}}{\partial t^{2}} .
$$

При этом магнитное и электрическое поля определятся соотношениями

$$
\mathbf{H}=\operatorname{rot} \mathbf{A}_{H} ; \quad \mathbf{E}=-\mu \frac{\partial \mathbf{A}_{H}}{\partial t} .
$$

Вектор Пойнтинга в данном случае имеет вид:

$$
\mathbf{P}=-\mu\left[\frac{\partial \mathbf{A}_{H}}{\partial t} \times \operatorname{rot} \mathbf{A}_{H}\right] .
$$

Пространственная производная $\operatorname{rot} \mathbf{A}_{H}$ и производная по времени сязаны волновым уравненрием (4.7).

Можно поступить иначе, введя электрические и магнитные токи

$$
\mathbf{j}_{E}=\operatorname{rot} \mathbf{H}, \mathbf{j}_{H}=\operatorname{rot} \mathbf{E} .
$$

Для этих токов тоже могут быть записаны уравнения:

$$
\operatorname{rot} \mathbf{j}_{H}=-\mu \frac{\partial \mathbf{j}_{E}}{\partial t}, \operatorname{rot} \mathbf{j}_{E}=\varepsilon \frac{\partial \mathbf{j}_{H}}{\partial t} .
$$

Эта система по своему виду и заключенной в ней информации ничем не отличается от уравнений Максвелла, и можно считать, что в пространстве распространяются магнитные или электрические токи. И решение задачи распространения при помощи данного метода опять будет содержать в себе полную информацию о процессах распространения.

Рассмотренный процесс введения новых векторных полей можно распространять в обе стороны до бесконечности, вводя все новые векторные поля. Естественно, при этом следует вводить и дополнительные калибровки. Таким образом, существует бесконечное множество возможных записей электродинамических законов, но все они равноценны по заключенной в них информации. Такой подход был впервые продемонстрирован в $[9,10]$. 


\section{ГЛАВА 2}

\section{РОЛЬ И МЕСТО КИНЕТИЧЕКОЙ ИНДУКТИВНОСТИ ЗАРЯДОВ В СОВРЕМЕННОЙ ЭЛЕКТРОДИНАМИКЕ}

Классическая электродинамика материальных сред представляет собой один из важнейших разделов физики не только по своей теоретической, но и, в не меньшей мере, практической значимости. Тем не менее, традиционное рассмотрение даже такого основополагающего для нее вопроса, как частотная дисперсия электромагнитных волн [11-14], не обходится без существенных недоработок и слабых мест.

\section{§ 5. Кто и как ввёл частотную дисперсию диэлектрической проницаемости материальных сред}

Общеизвестно, что физика является количественной наукой, основанной на физическом эксперименте, опирающемся на измерения, то есть сравнение характеристик исследуемых явлений с определенными эталонами. Для этого в физике вводятся физические величины, физические единицы их измерения и измерительные приборы. Экспериментально полученные количественные зависимости позволяют использовать для своей обработки математические методы и строить теоретические, то есть математические модели изучаемых явлений. Основными составляющими математической модели являются функциональные зависимости, связывающие между собой различные переменные принятой модели.

Такими переменными могут быть не только физические величины, но и параметры математической модели (кратко - математические параметры), играющие в модели вспомогательную роль. Математические модели позволяют, среди всего прочего, количественно формулировать (то есть формулировать на языке математики) физические законы, но при этом важно, что при записи физического закона можно использовать в качестве переменных только физические величины. Это позволяет рассматривать физический смысл законов, так как математические параметры, в отличие от физических величин, физическим смыслом не наделены. В частности, математический параметр может выражаться комплексным числом (например, комплексная диэлектрическая проницаемость, используемая в методе комплексных амплитуд), в то время как физическая величина не может быть комплекснозначной (например, относительная диэлектрическая проницаемость среды). Приведенные примеры тривиальны, но в случаях, когда последовательный анализ физического смысла зависимостей затруднителен, может возникать путаница в разграничении физических величин и математических параметров.

Всем хорошо известно такое явление, как радуга. Специалисту по электродинамике ясно, что ее возникновение связано с зависимостью от частоты фазовой скорости электромагнитных волн, проходящих через капли 
дождя (частотная дисперсия волны). Поскольку вода является диэлектриком, то при объяснении этого явления Дж. Хевисайд и Р. Вуд предположили, что такая дисперсия связана с частотной дисперсией (зависимостью от частоты) диэлектрической проницаемости воды. С тех пор эта точка зрения является господствующей [11-16].

Напомним, что относительная диэлектрическая проницаемость среды это физическая величина, характеризующая диэлектрические свойства среды и показывающая, во сколько раз сила взаимодействия двух электрических зарядов в этой среде меньше, чем в вакууме. Частота же характеризует отдельную монохроматическую составляющую электромагнитной волны и прямого отношения к электрическому полю заряда не имеет. Следовательно, говоря о частотной дисперсии диэлектрической проницаемости, Дж. Хевисайд и Р. Вуд имели в виду зависимость от частоты не физической величины относительной диэлектрической проницаемости среды, а какого-то нового математического параметра.

Конечно, во избежание путаницы, лучше было бы такую диэлектрическую проницаемость назвать как-то по-другому (например, эффективной диэлектрической проницаемостью), подобно тому, как комплексную диэлектрическую проницаемость не называют относительной диэлектрической проницаемостью. Но почему-то эти знаменитые ученые этого не сделали, видимо, просто надеясь на то, что недоразумений не будет. Тем более, что еще Максвелл отмечал [1], что относительная диэлектрическая проницаемость является константой.

Как родилась идея дисперсии диэлектрической и магнитной проницаемости, и какой путь она прошла, красочно характеризует цитата из монографии известных специалистов в области физики плазмы [11]: «Сам Дж. Максвелл при формулировке уравнений электродинамики материальных сред считал, что диэлектрическая и магнитная проницаемости являются постоянными величинами (по этой причине они длительное время считались постоянными величинами). Значительно позже, уже в начале этого столетия при объяснении оптических дисперсионных явлений (в частности явления радуги) Дж. Хевисайд и Р. Вул показали, что диэлектрическая и магнитная проницаемости являются функциями частоты. А совсем недавно, в середине 50-х годов, физики пришли к выводу, что эти величины зависят не только от частоты, но и от волнового вектора. По сути, это была радикальная ломка существующих представлений. Насколько серьезной она была, характеризует случай, который произошел на семинаре Л.Д. Ландау в 1954 г. Во время доклада А.И. Ахиезера на эту тему Ландау вдруг воскликнул, перебив докладчика: «Это бред, поскольку показатель преломления не может быть функцией показателя преломления». Заметьте, что это сказал Л.Д. Ландау - один из выдающихся физиков нашего времени» (конец цитаты). Из приведенной цитаты непонятно, что именно имел в виду Ландау. Однако последующие его публикации говорят о том, что он эту концепцию принял [12]. И опять почему-то, вслед за Хевисайдом и Вудом, Ландау не ввел новое название для нового математического параметра. Вряд ли такой выдающийся физик XX века мог не понимать такой 
очевидной вещи, что речь идет именно о новом математическом параметре. Скорее, так же считал, что недоразумений не будет.

Подобное же рассмотрение имело место в ряде фундаментальных работ по электродинамике [11-16], в результате чего в физике прочно закрепилось такое понятие, как частотная дисперсия диэлектрической проницаемости материальных сред и, в частности, плазмы. Распространение этой концепции на диэлектрики привело к представлениям о том, что их диэлектрическая проницаемость тоже зависит от частоты. Имеется множество публикаций, начиная с таких известных учёных, как Друде, Вуд, Хевисайд, Ландау, Гинзбург [11-16], и заканчивая Большой Советской Энциклопедией, где говорится, что диэлектрическая проницаемость плазмы и диэлектриков зависит от частоты.

К сожалению, это вызвало много недоразумений. Так, многие специалисты не могут поверить в то, что физическая величина относительной диэлектрической проницаемости плазмы равна относительной диэлектрической проницаемости вакуума, а дисперсия физической величины диэлектрической проницаемости диэлектриков отсутствует. Главный же негативный момент здесь состоит в том, что не акцентируется внимание исследователей на актуальности совершенствования математических моделей дисперсии электромагнитных волн в направлении перехода от рассмотрения математического параметра под названием диэлектрической проницаемости к рассмотрению физической величины относительной диэлектрической проницаемости.

Построение таких моделей дисперсии возможно только на основе глубокого понимания физического смысла происходящих процессов. Но именно такие модели могут описывать те стороны явления, которые ранее оказывались недоступными для теоретических исследований. Далее мы покажем, как надлежащее определение роли и места кинетической индуктивности зарядов в электродинамике позволяет при рассмотрении явления дисперсии электромагнитных волн ограничиться использованием только физической величины относительной диэлектрической проницаемости среды без привлечения соответствующих математических параметров.

\section{§ 6. Плазмоподобные среды}

Под бездиссипативными плазмоподобными средами будем понимать такие, в которых заряды движутся без потерь. В первом приближении к ним могут быть отнесены сверхпроводники, свободные электроны или ионы в вакууме (в дальнейшем проводники). Для электронов в указанных средах в отсутствии магнитного поля уравнение движения заряда имеет вид:

$$
m \frac{d \mathbf{v}}{d t}=e \mathbf{E},
$$

где $m$ и $e$ - масса и заряд электрона, $\mathbf{E}$ - напряженность электрического поля, $\mathbf{v}-$ скорость движения заряда. 
В данном уравнении считается, что заряд электрона отрицательный. В [16] показано, что это уравнение может быть распространено на случай движения электронов в горячей плазме.

Используя взаимосвязь плотностей тока и электронов

$$
\mathbf{j}=n e \mathbf{v}
$$

из (6.1) получим плотность тока проводимости

$$
\mathbf{j}_{L}=\frac{n e^{2}}{m} \int \mathbf{E} d t .
$$

Введя согласно [8,9,17-19] удельную кинетическую индуктивность носителей заряда, существование которой связано с инерционными свойствами носителей заряда, имеющих массу

$$
L_{k}=\frac{m}{n e^{2}},
$$

запишем равенство (6.3) в виде

$$
\mathbf{j}_{L}=\frac{1}{L_{k}} \int \mathbf{E} d t .
$$

Для случая гармонических полей $\mathbf{E}=\mathbf{E}_{0} \sin \omega t$ (6.5) запишется в виде:

$$
\mathbf{j}_{L}=-\frac{1}{\omega L_{k}} \mathbf{E}_{0} \cos \omega t \text {. }
$$

Здесь и далее, как правило, используется не комплексная, а вещественная форма записи электродинамических формул по причине ее наглядности в отражении фазовых соотношений между векторами, представляющими электрические поля и плотности токов. Из (6.5)-(6.6) видно, что плотность тока $\mathbf{j}_{L}$ описывает индуктивный ток, т.к. его фаза запаздывает по отношению к напряжённости электрического поля на угол $\pi / 2$.

Если заряды находятся в вакууме, то при нахождении суммарного тока нужно дополнительно учитывать ток смещения с плотностью

$$
\mathbf{j}_{\varepsilon}=\varepsilon_{0} \frac{\partial \mathbf{E}}{\partial t}=\omega \varepsilon_{0} \mathbf{E}_{0} \cos \omega t
$$

Видно, что этот ток носит ёмкостной характер, т.к. его фаза на $\pi / 2$ опережает фазу напряжённости электрического поля. Таким образом, суммарная плотность тока составит [17-22]:

$$
\mathbf{j}_{\Sigma}=\varepsilon_{0} \frac{\partial \mathbf{E}}{\partial t}+\frac{1}{L_{k}} \int \mathbf{E} d t
$$

или для случая гармонических полей

$$
\mathbf{j}_{\Sigma}=\left(\omega \varepsilon_{0}-1 /\left(\omega L_{k}\right)\right) \mathbf{E}_{0} \cos \omega t .
$$

Если электроны находятся в материальной среде, то в общем случае следует ещё учитывать наличие положительно заряженных ионов, но в частном случае быстропеременных полей их наличие можно не учитывать в связи со значительным превышением массы ионов над массой электронов.

В (6.7) величина в скобках - суммарная реактивная проводимость среды $\sigma_{\Sigma}$, складывающаяся из емкостной $\sigma_{C}$ и индуктивной $\sigma_{L}$ проводимости 


$$
\sigma_{\Sigma}=\sigma_{C}+\sigma_{L}=\omega \varepsilon_{0}-1 /\left(\omega L_{k}\right) .
$$

Соотношение (6.7) можно переписать и по-другому:

$$
\mathbf{j}_{\Sigma}=\omega \varepsilon_{0}\left(1-\omega_{0}^{2} / \omega^{2}\right) \mathbf{E}_{0} \cos \omega t,
$$

где $\omega_{0}=1 / \sqrt{L_{k} \varepsilon_{0}}$ - плазменная частота.

Так получилась скалярная величина

$$
\varepsilon^{*}(\omega)=\sigma_{\Sigma} / \omega=\varepsilon_{0}\left(1-\omega_{0}^{2} / \omega^{2}\right)=\varepsilon_{0}-1 /\left(\omega^{2} L_{k}\right),
$$

которая в научной литературе, в частности, в работах по физике плазмы [11-16], названа диэлектрической проницаемостью плазмы. Если трактовать эту величину, как абсолютную диэлектрическую проницаемость плазмы в том смысле, что ее отношение к электрической постоянной дает физическую величину относительной диэлектрической проницаемости плазмы, то получится, что физическая величина относительной диэлектрическая проницаемости плазмы зависит от частоты. В предыдущем параграфе было отмечено, что это неверно, а полученная величина является неким математическим параметром, который необходимо отличать от абсолютной и относительной диэлектрической проницаемости. В отличие от абсолютной диэлектрической проницаемости, которую удобно называть также в более развернутом варианте наименования физической абсолютной диэлектрической проницаемостью, введенную величину назовем эффективной абсолютной диэлектрической проницаемостью. Аналогично, в отличие от относительной диэлектрической проницаемости, которую удобно называть также в более развернутом варианте наименования физической относительной диэлектрической проницаемостью, отношение введенной величины к электрической постоянной назовем эффективной относительной диэлектрической проницаемостью. Если физические абсолютная и относительная диэлектрические проницаемости среды не зависят от частоты, то эффективные абсолютная и относительная диэлектрические проницаемости среды от частоты зависят.

Важно отметить, что эффективная абсолютная диэлектрическая проницаемость плазмы оказалась сборным математическим параметром, в который одновременно входит электрическая постоянная и удельная кинетическая индуктивность зарядов [22-24].

Для дальнейшей конкретизации рассмотрения дисперсии электромагнитных волн определим понятия физических диэлектрических проницаемостей среды (абсолютной и относительной) для случая переменных полей. Входящая во второе уравнение Максвелла суммарная плотность токов (в дальнейшем для краткости будем употреблять слово «ток» вместо «плотность тока») в любой среде складывается только из следующих трех составляющих, зависящих от электрического поля:

1) ток резистивных потерь (синфазен электрическому полю);

2) емкостной ток, называемый током смещения (определяется первой производной электрического поля по времени и опережает напряженность электрического поля по фазе на $\pi / 2$ ); 
3) ток проводимости (определяется интегралом от электрического поля по времени и отстает от электрического поля по фазе на $\pi / 2$ ).

Все эти составляющие должны присутствовать в любых немагнитных средах с тепловыми потерями. Поэтому естественно определить физическую абсолютную диэлектрическую проницаемость любой среды как коэффициент перед членом, определяемым производной электрического поля по времени во втором уравнении Максвелла. Следует учесть, что эта диэлектрическая проницаемость не может быть отрицательной, так как определяемая через неё энергия электрических полей всегда неотрицательна. Соответственно, физическая относительная диэлектрическая проницаемость равна отношению физической абсолютной диэлектрической проницаемости к электрической постоянной. Отметим тривиальное общее правило для эффективной и для физической диэлектрической проницаемости - относительная проницаемость всегда равна отношению абсолютной проницаемости к электрической постоянной, так что слово «абсолютная» или «относительная» будем для краткости по возможности опускать.

Предлагаемая математическая модель дисперсии электромагнитных волн в плазме отличается от ранее известных тем, что использует не эффективную, а физическую диэлектрическую проницаемость плазмы. Это становится возможным за счет учета кинетической индуктивности зарядов на основе углубленного понимания физического смысла дисперсии. В результате, предлагаемая модель позволяет учитывать начальные условия при решении интегро-дифференциального уравнения для тока посредством введения соответствующей постоянной интегрирования.

При традиционном же рассмотрении физическая диэлектрическая проницаемость плазмы в переменных электрических полях не определяется и даже ток не раскладывается на ток смещения и ток проводимости, один из которых определяется электрической постоянной и производной функции электрического поля, а другой определяется удельной кинетической индуктивностью и интегралом от электрического поля. До некоторой степени «сваливание токов в общую кучу» оправдано, так как производная и интеграл функции гармонического колебания различаются лишь знаком. Однако при этом теряется постоянная интегрирования, которая необходима для учёта начальных условий при решении интегро-дифференциального уравнения, определяющего плотность тока в материальной среде.

Разделение токов в предлагаемой модели проясняет физику явления. Один из этих двух противофазных конкурирующих токов зависит от частоты линейно, другой - обратно пропорционально частоте. При низких частотах преобладает ток проводимости, при высоких - ток смещения. На плазменной частоте токи равны и вступают в резонанс друг с другом.

Аналогично введению эффективной диэлектрической проницаемости можно ввести зависящую от частоты эффективную (отличную от физической) кинетическую индуктивность

$$
L_{k}^{*}(\omega)=\frac{L_{k}}{\left(1-\omega^{2} / \omega_{0}^{2}\right)}=\frac{L_{k}}{1-\varepsilon_{0} \omega^{2} L_{k}},
$$


записав соотношение (6.7) в виде:

$$
\mathbf{j}_{\Sigma}=-\frac{\left(1-\omega^{2} / \omega_{0}^{2}\right)}{\omega L_{k}} \mathbf{E}_{0} \cos \omega t .
$$

Параметры $\varepsilon^{*}(\omega), L_{k}^{*}(\omega)$ дают две эквивалентные формы (6.7):

$$
\mathbf{j}_{\Sigma}=\omega \varepsilon^{*}(\omega) \mathbf{E}_{0} \cos \omega t, \mathbf{j}_{\Sigma}=-\frac{1}{\omega L_{k}^{*}(\omega)} \mathbf{E}_{0} \cos \omega t .
$$

Первый из этих параметров равен отношению суммарной реактивной проводимости среды к частоте, а второй равен взятой со знаком «минус» обратной величине произведения частоты и реактивной проводимости среды:

$$
\varepsilon^{*}(\omega)=\sigma_{\Sigma} / \omega, L_{k}^{*}(\omega)=-1 /\left(\omega \sigma_{\Sigma}\right) .
$$

Их нельзя подставлять в формулы для энергии электрических полей

$$
W_{E}=\varepsilon_{0} E_{0}^{2} / 2
$$

и кинетической энергии носителей зарядов

$$
W_{j}=L_{k} j_{0}^{2} / 2
$$

просто потому, что в этих формулах фигурируют не эффективные, а соответствующие физические величины. Нетрудно показать, что полная удельная энергия может быть получена из соотношения

$$
W_{\Sigma}=\frac{1}{2} \cdot \frac{d\left(\omega \varepsilon^{*}(\omega)\right)}{d \omega} E_{0}^{2}
$$

из которого получаем

$$
W_{\Sigma}=\frac{1}{2} \varepsilon_{0} E_{0}^{2}+\frac{1}{2} \frac{1}{\omega^{2} L_{k}} E_{0}^{2}=\frac{1}{2} \varepsilon_{0} E_{0}^{2}+\frac{1}{2} L_{k} j_{0}^{2} .
$$

Тот же результат получим, воспользовавшись формулой

$$
W=\frac{1}{2} \frac{d\left[\frac{1}{\omega L_{k}^{*}(\omega)}\right]}{d \omega} E_{0}^{2} .
$$

Видно, что удельная энергия состоит из потенциальной энергии электрических полей и кинетической энергии носителей зарядов.

Волновое уравнение следует из системы уравнений Максвелла, полностью описывающей электродинамику бездиссипативных проводников:

$$
\operatorname{rot} \mathbf{E}=-\mu_{0} \frac{\partial \mathbf{H}}{\partial t}, \quad \operatorname{rot} \mathbf{H}=\varepsilon_{0} \frac{\partial \mathbf{E}}{\partial t}+\frac{1}{L_{k}} \int \mathbf{E} d t,
$$

где $\varepsilon_{0}$ и $\mu_{0}$ - электрическая и магнитная постоянные.

Из (6.10) получаем

$$
\operatorname{rot} \operatorname{rot} \mathbf{H}+\mu_{0} \varepsilon_{0} \frac{\partial^{2} \mathbf{H}}{\partial t^{2}}+\frac{\mu_{0}}{L_{k}} \mathbf{H}=0 .
$$

Если поля не зависят от времени, (6.11) переходит в уравнение Лондонов

$$
\operatorname{rot} \operatorname{rot} \mathbf{H}+\frac{\mu_{0}}{L_{k}} \mathbf{H}=0,
$$

где $\lambda_{L}^{2}=L_{k} / \mu_{0}-$ лондоновская глубина проникновения. 
Таким образом, уравнения Лондонов [25] являются частным случаем уравнения (6.11) и не учитывают токов смещения в среде. Поэтому из этих уравнений нельзя получить волновые уравнения, описывающие процессы распространения электромагнитных волн в сверхпроводниках.

Для электрических полей волновое уравнение примет вид:

$$
\operatorname{rot} \operatorname{rot} \mathbf{E}+\mu_{0} \varepsilon_{0} \frac{\partial^{2} \mathbf{E}}{\partial t^{2}}+\frac{\mu_{0}}{L_{k}} \mathbf{E}=0 \text {. }
$$

Для постоянных электрических полей имеем:

$$
\operatorname{rot} \operatorname{rot} \mathbf{E}+\frac{\mu_{0}}{L_{k}} \mathbf{E}=0 .
$$

Следовательно, постоянные электрические поля проникают в сверхпроводник таким же образом, как и магнитные, убывая по экспоненциальному закону. Плотность же тока при этом растёт по линейному закону

$$
\mathbf{j}_{L}=\frac{1}{L_{k}} \int \mathbf{E} d t .
$$

Видно, что физическая абсолютная диэлектрическая проницаемость данной среды связана с накоплением потенциальной энергии, не зависит от частоты и равна физической абсолютной диэлектрической проницаемости вакуума, то есть электрической постоянной. Кроме того, такую среду характеризует ещё и (физическая) кинетическая индуктивность носителей зарядов, связанная с накоплением кинетической энергии.

В отличие от общепринятой методики [12-13] рассмотрения распространения электромагнитных волн в проводящих средах, не имеющих потерь, предлагаемая методика не требует введения вектора поляризации, а основывается на уравнении движения, и при этом во втором уравнении Максвелла выписываются все составляющие плотностей токов в явном виде.

Для дальнейшего понимания физической природы дисперсии привлечём радиотехнический метод эквивалентных схем, наглядно представляющий не только радиотехнические элементы с сосредоточенными и распределёнными параметрами, но и материальные среды. Как будет показано ниже, согласно этому методу, единичный объём проводника или плазмы по своим электродинамическим характеристикам эквивалентен параллельному резонансному контуру с сосредоточенными параметрами. Рассмотрим параллельный резонансный контур с параллельным включением емкости $C$ и индуктивности $L$. Связь между напряжением $U$, приложенным к контуру, и суммарным током $I_{\Sigma}$, текущем через такую цепь, имеет вид:

$$
I_{\Sigma}=I_{C}+I_{L}=C \frac{d U}{d t}+\frac{1}{L} \int U d t,
$$

где $I_{C}=C \frac{d U}{d t}, I_{L}=\frac{1}{L} \int U d t-$ токи, текущие через емкость и индуктивность.

Для переменного напряжения по гармоническому закону $U=U_{0} \sin \omega t$ получаем:

$$
I_{\Sigma}=(\omega C-1 /(\omega L)) U_{0} \cos \omega t
$$


В (6.12) величина в скобках есть суммарная реактивная проводимость $\sigma_{\Sigma}$ цепи, состоящая из емкостной $\sigma_{C}$ и индуктивной $\sigma_{L}$ проводимости

$$
\sigma_{\Sigma}=\sigma_{C}+\sigma_{L}=\omega C-1 /(\omega L) .
$$

Соотношение (6.12) можно переписать следующим образом:

$$
I_{\Sigma}=\omega C\left(1-\omega_{0}^{2} / \omega^{2}\right) U_{0} \cos \omega t,
$$

где $\omega_{0}^{2}=1 /(L C)$ - резонансная частота параллельного контура.

Как и в случае проводников, можно ввести новый математический параметр эффективной емкости

$$
C^{*}(\omega)=C\left(1-\omega_{0}^{2} / \omega^{2}\right)=C-1 /\left(\omega^{2} L\right),
$$

зависящей от частоты, емкости и даже индуктивности и равной отношению реактивной проводимости цепи к частоте. И опять нужно этот математический параметр отличать от физической емкости, которую принято называть просто емкостью, и которая является не математическим параметром, а физической величиной.

Соотношение (6.12) можно переписать и по-другому:

$$
I_{\Sigma}=-\frac{\left(1-\omega^{2} / \omega_{0}^{2}\right)}{\omega L} U_{0} \cos \omega t
$$

введя новый математический параметр эффективной индуктивности

$$
L^{*}(\omega)=\frac{L}{\left(1-\omega^{2} / \omega_{0}^{2}\right)}=\frac{L}{1-\omega^{2} L C},
$$

равный взятой со знаком «минус» обратной величине произведения суммарной реактивной проводимости и частоты.

Используя выражения (6.13)-(6.14), запишем:

$$
\begin{gathered}
I_{\Sigma}=\omega C^{*}(\omega) U_{0} \cos \omega t, \\
I_{\Sigma}=-\left(1 /\left(\omega L^{*}(\omega)\right)\right) U_{0} \cos \omega t .
\end{gathered}
$$

Соотношения (6.15) и (6.16), используя разные параметры $C *(\omega)$ и $L *(\omega)$, эквивалентны, и каждое из них полностью характеризует цепь.

Накапливаемая в физической ёмкости и физической индуктивности энергия определяется из соответствующих соотношений

$$
\begin{aligned}
& W_{C}=C U_{0}^{2} / 2, \\
& W_{L}=L I_{0}^{2} / 2 .
\end{aligned}
$$

Если в (6.17)-(6.18) вместо физических емкости и индуктивности подставить эффективные величины (6.13)-(6.14), то энергия может стать отрицательной. Возникает так называемая проблема отрицательной энергии, присущая целому ряду математических моделей частотной дисперсии, в том числе уравнениям Клейна-Гордона для скалярных массивных частиц и Дирака для фермионов в квантовой физике. В случае же параллельного резонансного контура очевидно, что указанная проблема обязана своему возникновению некорректной замене физических величин на соответствующие эффективные математические параметры. Это дает определенные 
ориентиры для более глубокого исследования проблемы отрицательной энергии в разных моделях частотной дисперсии, в том числе квантовых, но эти вопросы уже выходят за рамки тематики данной монографии.

Легко видеть, что суммарная энергия, накопленная в контуре, может быть выражена взаимно эквивалентными равенствами:

$$
\begin{aligned}
& W_{\Sigma}=\frac{1}{2} \frac{d \sigma_{X}}{d \omega} U_{0}^{2}, \\
& W_{\Sigma}=\frac{1}{2} \frac{d\left[\omega C^{*}(\omega)\right]}{d \omega} U_{0}{ }^{2}, \\
& W_{\Sigma}=\frac{1}{2} \frac{d\left(\frac{1}{\omega L^{*}(\omega)}\right)}{d \omega} U_{0}{ }^{2} .
\end{aligned}
$$

Любое из равенств (6.19)-(6.21) дает одинаковый результат:

$$
W_{\Sigma}=C U_{0}^{2} / 2+L I_{0}^{2} / 2,
$$

где $U_{0}-$ есть амплитуда напряжения на ёмкости, а $I_{0}-$ амплитуда тока, текущего через индуктивность.

Следовательно, параллельный резонансный контур можно математически моделировать с трех взаимно эквивалентных точек зрения:

1) контур образуют физическая емкость и физическая индуктивность;

2) контур описывается частотозависимой эффективной емкостью;

3) контур описывается частотозависимой эффективной индуктивностью.

В квазистатическом режиме электродинамические процессы в проводниках подобны процессам в параллельном резонансном контуре с сосредоточенными параметрами. Соотношения для параллельного резонансного контура идентичны соотношениям для проводников при замене: $E_{0} \rightarrow U_{0}$ $, j_{0} \rightarrow I_{0}, \varepsilon_{0} \rightarrow C, L_{k} \rightarrow L$. Таким образом, единичный объём проводника, при однородном распределении электрических полей и плотностей токов в нём, эквивалентен параллельному резонансному контуру с указанными сосредоточенными параметрами. При этом физическая ёмкость такого контура численно равна физической абсолютной диэлектрической проницаемости вакуума (электрической постоянной), а физическая индуктивность равна удельной кинетической индуктивности зарядов.

Данный подход не требует введения в рассмотрение вектора поляризации в проводниках в отличие от общепринятой методики [12-15]. В частности, параграф 59 работы [12] начинается словами: «Мы переходим теперь к изучению важнейшего вопроса о быстропеременных электрических полях, частоты которых не ограничены условием малости по сравнению с частотами, характерными для установления электрической и магнитной поляризации вещества» (конец цитаты). Эти слова означают, что рассматривается та область частот, где в связи с наличием инерционных свойств носителей зарядов поляризация вещества не будет достигать её статических значений. При дальнейшем рассмотрении вопроса делается заключение, что «в любом переменном поле, в том числе при наличии дисперсии вектор поляризации $\mathbf{P}=\mathbf{D}-\varepsilon_{0} \mathbf{E}$ (здесь и далее все цитируемые формулы 
записываются в системе СИ) сохраняет свой физический смысл электрического момента единицы объёма вещества» (конец цитаты). Приведём ещё одну цитату из [12]: «Оказывается возможным установить справедливый для любых тел (безразлично - металлов или диэлектриков) предельный вид функции $\varepsilon(\omega)$ при больших частотах. Именно частота поля должна быть велика по сравнению с «частотами» движения всех (или, по крайней мере, большинства) электронов в атомах данного вещества. При соблюдении этого условия можно при вычислении поляризации вещества рассматривать электроны как свободные, пренебрегая их взаимодействием друг с другом и с ядрами атомов» (конец цитаты).

Далее, как и в данной работе, в [12] записывается уравнение движения свободного электрона в переменном электрическом поле

$$
m \frac{d \mathbf{v}}{d t}=e \mathbf{E},
$$

откуда находится его смещение

$$
\mathbf{r}=-\frac{e \mathbf{E}}{m \omega^{2}} .
$$

Затем утверждается, что поляризация $\mathbf{P}$ есть дипольный момент единицы объёма и полученное смещение вставляется в поляризацию

$$
\mathbf{P}=n e \mathbf{r}=-\frac{n e^{2} \mathbf{E}}{m \omega^{2}} .
$$

В данном случае рассматривается точечный заряд, и эта операция означает введение электрического дипольного момента для двух точечных зарядов с противоположными знаками, расположенными на расстоянии $\mathbf{r}$,

$$
\mathbf{p}_{e}=-e \mathbf{r},
$$

где вектор $\mathbf{r}$ направлен от положительного заряда к отрицательному. Этот шаг не представляется достаточно обоснованным, поскольку рассматривается точечный электрон, и чтобы говорить об электрическом дипольном моменте, нужно иметь в этой среде для каждого электрона парный заряд противоположного знака, отнесённый от него на расстояние $\mathbf{r}$. В данном же случае рассматривается газ свободных электронов, в котором отсутствуют заряды противоположных знаков. Далее следует стандартная процедура, когда введённый таким необоснованным способом вектор поляризации вводится в диэлектрическую проницаемость

$$
\mathbf{D}=\varepsilon_{0} \mathbf{E}+\mathbf{P}=\varepsilon_{0} \vec{E}-\frac{n e^{2} \mathbf{E}}{m \omega^{2}}=\varepsilon_{0}\left(1-\frac{1}{\varepsilon_{0} L_{k} \omega^{2}}\right) \mathbf{E} .
$$

А поскольку плазменная частота определяется соотношением

$$
\omega_{p}{ }^{2}=1 /\left(\varepsilon_{0} L_{k}\right),
$$

сразу записывается вектор индукции

$$
\mathbf{D}=\varepsilon_{0}\left(1-\omega_{p}^{2} / \omega^{2}\right) \mathbf{E} .
$$

При этом получается, что коэффициент пропорциональности

$$
\varepsilon(\omega)=\varepsilon_{0}\left(1-\omega_{p}^{2} / \omega^{2}\right)
$$


между электрическим полем и электрической индукцией, названный диэлектрической проницаемостью, зависит от частоты, а вслед за ним и электрическая индукция была объявлена зависящей от частоты [12-16]. Но, как было показано выше, этот математический параметр является не физической абсолютной диэлектрической проницаемостью, а отношением суммарной реактивной проводимости среды к частоте.

Далее в § 61 работы [12] рассматривается вопрос об энергии электрического и магнитного поля в средах, обладающих дисперсией. При этом делается вывод о том, что соотношение для энергии таких полей

$$
W=\left(\varepsilon E_{0}^{2}+\mu H_{0}^{2}\right) / 2,
$$

имеющего точный термодинамический смысл в обычных средах, при наличии дисперсии так истолковано быть не может. Эти слова означают, что знания реальных электрических и магнитных полей в диспергирующей среде недостаточно для определения разности внутренней энергии в единице объёма вещества при наличии полей и в их отсутствии. После такого утверждения приводится формула, дающая тот же результат для вычисления удельной энергии электрических и магнитных полей при наличии дисперсии, что и предлагаемый в данной монографии подход:

$$
W=\frac{1}{2} \frac{d(\omega \varepsilon(\omega))}{d \omega} E_{0}^{2}+\frac{1}{2} \frac{d(\omega \mu(\omega))}{d \omega} H_{0}^{2} .
$$

Первое слагаемое в правой части (6.23) соответствует (6.9), а значит представляет собой полную энергию, включающую не только потенциальную энергию электрических полей, но и кинетическую энергию движущихся зарядов. Это подтверждает вывод о невозможности толкования именно формулы (6.22), как внутренней энергии электрических и магнитных полей в диспергирующих средах, хотя такая интерпретация в рассмотренных средах в принципе возможна и состоит в том, что при определении удельной энергии, как термодинамического параметра, нужно учитывать не только электрическое поле, которое накапливает потенциальную энергию, но и ток электронов проводимости, которые в связи с наличием массы, накапливают кинетическую энергию движения зарядов (6.8).

\section{§ 7. Поперечный плазменный резонанс}

Разработка математической модели дисперсии электромагнитных волн в проводящих средах, использующей физическую диэлектрическую проницаемость плазмы, позволяет выдвинуть теоретически обоснованную гипотезу о существовании нового физического явления. Оно может быть названо поперечным плазменным резонансом в незамагниченной плазме. Это явление не только представляет большой теоретический интерес, но и может иметь важные технические приложения [26, 27].

Ленгмюровский резонанс является продольным и потому не может излучать поперечные радиоволны. Однако при взрывах ядерных зарядов, в результате которых образуется очень горячая плазма, имеет место электро- 
магнитное излучение в очень широком диапазоне частот, вплоть до длинноволнового радиодиапазона. Науке неизвестны физические механизмы, объясняющие возникновение такого излучения. О существовании в незамагниченной плазме каких-либо других резонансов, кроме ленгмюровского, ранее известно не было, но оказывается, что в ограниченной плазме может существовать поперечный резонанс, и частота такого резонанса совпадает с частотой ленгмюровского резонанса, т.е. эти резонансы являются вырожденными. Именно поперечный резонанс может быть причиной излучения радиоволн при взрывах ядерных зарядов. Для выяснения условий возбуждения такого резонанса рассмотрим длинную линию, состоящую из двух идеально проводящих плоскостей, как показано на рис. 2.

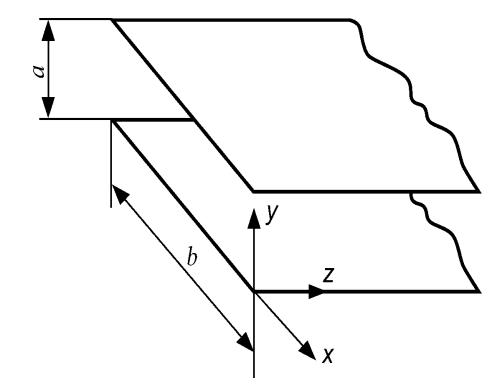

Рис. 2. Двухпроводная линия из двух идеально проводящих плоскостей.

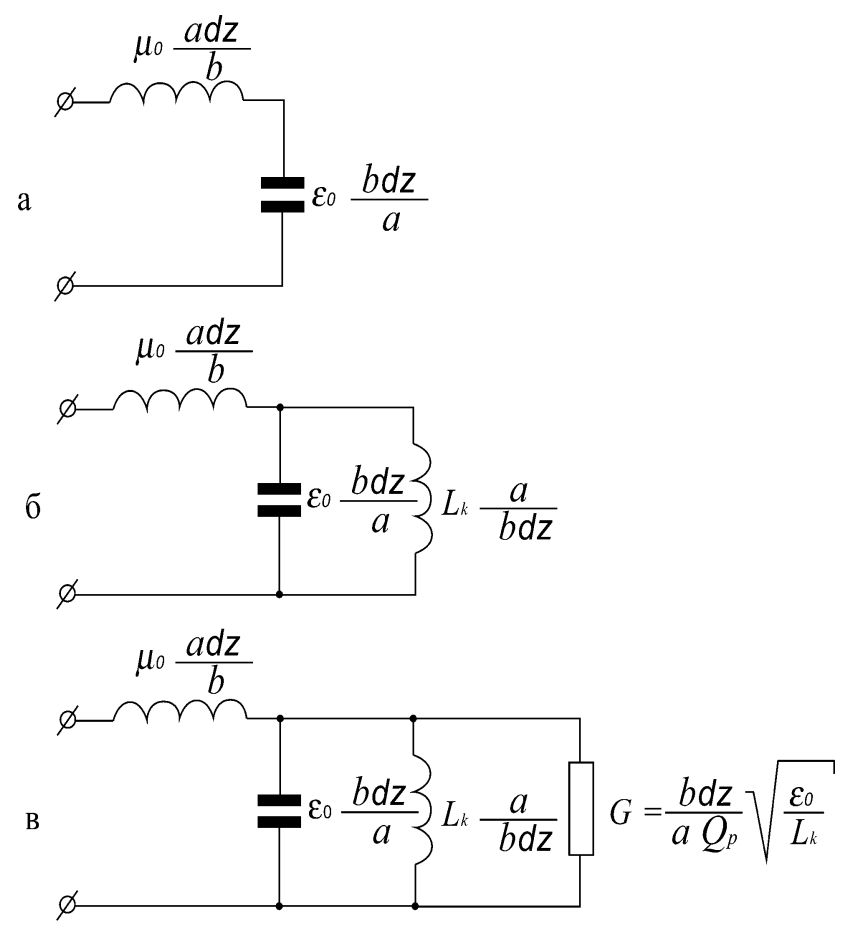

Рис. 3. а - эквивалентная схема отрезка двухпроводной линии;

б - эквивалентная схема отрезка двухпроводной линии, заполненной бездиссипативной плазмой;

в - эквивалентная схема отрезка двухпроводной линии, заполненной диссипативной плазмой

Погонная (приходящаяся на единицу длины) емкость и индуктивность такой линии без учёта краевых эффектов определяются соотношениями: 


$$
C_{0}=\varepsilon_{0} b / a \quad \text { и } \quad L_{0}=\mu_{0} a / b .
$$

Поэтому с ростом длины линии ее суммарные емкость $C_{\Sigma}=\varepsilon_{0}(b / a) z$ и индуктивность $L_{\Sigma}=\mu_{0}(a / b) z$ увеличиваются пропорционально длине.

Если в разомкнутую линию поместить плазму, носители заряда в которой могут двигаться без потерь, и в поперечном направлении пропустить через плазму ток I, то заряды в связи с наличием у них массы, двигаясь с определенной скоростью, будут накапливать кинетическую энергию. Заметим, что здесь не рассматриваются технические вопросы, как и каким образом можно удержать плазму между плоскостями линии. В данном случае рассматриваются только принципиальные вопросы, касающиеся рассматриваемого поперечного плазменного резонанса в незамагниченной плазме.

Поскольку поперечная плотность тока в такой линии равна

$$
j=I /(b z)=n e v,
$$

то суммарная кинетическая энергия движущихся зарядов равна

$$
W_{k \Sigma}=\frac{1}{2} \frac{m}{n e^{2}} a b z j^{2}=\frac{1}{2} \frac{m}{n e^{2}} \frac{a}{b z} I^{2} .
$$

Соотношение (7.1) связывает кинетическую энергию, накопленную в линии, с квадратом тока, поэтому коэффициент в правой части перед квадратом тока является суммарной кинетической индуктивностью линии:

$$
L_{k \Sigma}=\frac{m}{n e^{2}} \cdot \frac{a}{b z} \text {. }
$$

Таким образом, величина

$$
L_{k}=m /\left(n e^{2}\right)
$$

есть удельная кинетическая индуктивность зарядов. Она ранее вводилась другим способом (6.4). Соотношение (7.3) получено для случая постоянного тока, когда токовое распределение является однородным.

Для наглядности будем использовать метод эквивалентных схем. Так, схема отрезка линии длиной $d z$ показана на рис. 3 (a).

Из (7.2) видно, что в отличие от $C_{\Sigma}$ и $L_{\Sigma}$ величина $L_{k \Sigma}$ с ростом $z$ не увеличивается, а уменьшается. Связано это с тем, что с ростом $z$ количество параллельно включенных индуктивных элементов растет.

Эквивалентная схема участка линии, заполненной плазмой, в которой отсутствуют потери, показана на рис. 3 (б). Сама линия при этом будет эквивалентна параллельному контуру с сосредоточенными параметрами:

$$
C=\varepsilon_{0} b z / a, \quad L=L_{k} a /(b z),
$$

последовательно с которым включена индуктивность

$$
\mu_{0} a d z / b \text {. }
$$

Резонансная частота такого контура имеет вид:

$$
\omega_{\rho}^{2}=1 /(C L)=1 /\left(\varepsilon_{0} L_{k}\right)=n e^{2} /\left(\varepsilon_{0} m\right) .
$$

Получен очень интересный результат - резонансная частота рассмотренного макроскопического резонатора не зависит от его размеров. Может создаться впечатление, что это плазменный резонанс, т.к. полученное значе- 
ние резонансной частоты в точности соответствует значению частоты плазменного резонанса. Но известно, что такой резонанс характеризует продольные волны, в то время как в длинной линии имеют место только поперечные волны. В рассмотренном случае величина фазовой скорости в направлении $z$ равна бесконечности и волновой вектор $\vec{k}=0$.

Данный результат соответствует решению системы уравнений (6.10) для линии с заданной конфигурацией. При этом квадраты волнового числа, групповой и фазовой скорости определяются соотношениями:

$$
\begin{gathered}
k_{z}^{2}=\frac{\omega^{2}}{c^{2}}\left(1-\omega_{\rho}^{2} / \omega^{2}\right), \\
v_{g}^{2}=c^{2}\left(1-\omega_{\rho}^{2} / \omega^{2}\right), \\
v_{F}^{2}=c^{2} /\left(1-\omega_{\rho}^{2} / \omega^{2}\right),
\end{gathered}
$$

где $c=1 /\left(\mu_{0} \varepsilon_{0}\right)^{1 / 2}$ - скорость света в вакууме.

Для данного случая фазовая скорость электромагнитной волны бесконечна, что соответствует поперечному резонансу на плазменной частоте. Следовательно, в каждый момент времени распределение полей и токов в такой линии однородно и не зависит от координаты $z$, а ток в плоскостях линии в направлении $z$ отсутствует. Это означает, что индуктивность $L_{\Sigma}$ не оказывает влияния на электродинамические процессы в такой линии, а вместо проводящих плоскостей могут быть использованы любые плоскости или устройства, ограничивающие плазму сверху и снизу.

Из (7.4)-(7.6) легко видеть, что в точке $\omega=\omega_{p}$ имеет место поперечный резонанс с бесконечной добротностью. При наличии потерь в резонаторе будет иметь место затухание, а в длинной линии в этом случае $k_{z} \neq 0$, и в линии будет распространяться затухающая поперечная волна, направление распространения которой будет нормально направлению движения зарядов. Существование такого резонанса ранее другими авторами не описано.

Перед тем, как перейти к более подробному рассмотрению данного вопроса, остановимся на энергетических процессах, имеющих место в рассмотренной линии в случае отсутствия потерь.

Характеристическое сопротивление плазмы, дающее отношение поперечных компонент электрического и магнитного полей, имеет вид:

$$
Z=E_{y} / H_{x}=\mu_{0} \omega / k_{z}=Z_{0}\left(1-\omega_{\rho}^{2} / \omega^{2}\right)^{-1 / 2},
$$

где $Z_{0}=\sqrt{\mu_{0} / \varepsilon_{0}}$ - характеристическое (волновое) сопротивление вакуума.

Полученное значение $Z$ характерно для поперечных электрических волн в волноводах. При $\omega \rightarrow \omega_{p}$ имеем: $Z \rightarrow \infty, H_{x} \rightarrow 0$. В случае $\omega>\omega_{p}$ в плазме существует и электрическая, и магнитная составляющая поля.

Удельная энергия этих полей имеет вид:

$$
W_{E, H}=\frac{1}{2} \varepsilon_{0} E_{0 y}^{2}+\frac{1}{2} \mu_{0} H_{0 x}^{2} .
$$


Следовательно, энергия, заключенная в магнитном поле, в $\left(1-\omega_{\rho}^{2} / \omega^{2}\right)$ раз меньше заключенной в электрическом. Данное традиционное в электродинамике рассмотрение неполно, т.к. не учитывает еще один вид энергии кинетической энергии носителей заряда. Кроме волн электрического и магнитного полей, несущих электрическую и магнитную энергии, в плазме существует еще и третья - кинетическая волна, несущая кинетическую энергию носителей тока. Удельная энергия этой волны имеет вид:

$$
W_{k}=\frac{1}{2} L_{k} j_{0}^{2}=\frac{1}{2} \cdot \frac{1}{\omega^{2} L_{k}} E_{0}^{2}=\frac{1}{2} \varepsilon_{0} \frac{\omega_{\rho}^{2}}{\omega^{2}} E_{0}^{2} .
$$

Следовательно, полная удельная энергия записывается как

$$
W_{E, H, j}=\frac{1}{2} \varepsilon_{0} E_{0 y}^{2}+\frac{1}{2} \mu_{0} H_{0 x}^{2}+\frac{1}{2} L_{k} j_{0}^{2} .
$$

Итак, для нахождения полной энергии, заключённой в единице объема плазмы, учет только полей $E$ и $H$ недостаточен. В точке $\omega=\omega_{p}$ имеем:

$$
W_{H}=0 ; \quad W_{E}=W_{k},
$$

т.е. магнитное поле в плазме отсутствует, и плазма представляет макроскопический электромеханический резонатор с бесконечной добротностью, резонирующий на частоте $\omega_{p}$.

Поскольку при частотах $\omega>\omega_{p}$ волна, распространяющаяся в плазме, несет на себе три вида энергии (электрическую, магнитную и кинетическую), то такую волну можно назвать электромагнитокинетической. Кинетическая волна является волной плотности тока $\mathbf{j}=\left(1 / L_{k}\right) \int \mathbf{E} d t$. Эта волна сдвинута по отношению к электрической волне на фазовый угол $\pi / 2$.

До сих пор рассматривался физически нереализуемый случай, когда потери в плазме отсутствуют, что соответствует бесконечной добротности плазменного резонатора. Если потери имеются, причем совершенно не важно какими физическими процессами такие потери обусловлены, то добротность плазменного резонатора будет конечной величиной. Для такого случая уравнения Максвелла будут иметь вид:

$$
\operatorname{rot} \mathbf{E}=-\mu_{0} \frac{\partial \mathbf{H}}{\partial t}, \quad \operatorname{rot} \vec{H}=\sigma_{p . e f} \mathbf{E}+\varepsilon_{0} \frac{\partial \mathbf{E}}{\partial t}+\frac{1}{L_{k}} \int \mathbf{E} d t .
$$

Наличие потерь учитывается членом $\sigma_{p . e f} \mathbf{E}$. При этом обозначение ef подчеркивает важность самого факта существования потерь, а не их конкретного механизма. Величину $\sigma_{e f}$ определяет добротность плазменного резонатора. Для измерения $\sigma_{e f}$ следует выбрать отрезок линии длиной $z_{0}$, много меньшей длины волны в диссипативной плазме. Такой отрезок эквивалентен контуру с сосредоточенными параметрами:

$$
C=\varepsilon_{0} \frac{b z_{0}}{a}
$$




$$
\begin{aligned}
L & =L_{k} \frac{a}{b z_{0}} \\
G & =\sigma_{\rho . e f} \frac{b z_{0}}{a}
\end{aligned}
$$

где $G$ - проводимость, подключенная параллельно $C$ и $L$.

Проводимость и добротность в таком контуре связаны соотношением

$$
G=\sqrt{C / L} / Q_{\rho}
$$

откуда, учитывая (7.8) - (7.10), получаем:

$$
\sigma_{\rho . e f}=\sqrt{\varepsilon_{0} / L_{k}} / Q_{\rho} .
$$

Таким образом, измеряя собственную добротность такого плазменного резонатора, можно определить $\sigma_{p . e f}$. Используя (7.2) и (7.11), получим:

$$
\operatorname{rot} \mathbf{E}=-\mu_{0} \frac{\partial \mathbf{H}}{\partial t}, \quad \operatorname{rot} \mathbf{H}=\frac{1}{Q_{\rho}} \sqrt{\frac{\varepsilon_{0}}{L_{k}}} \mathbf{E}+\varepsilon_{0} \frac{\partial \mathbf{E}}{\partial t}+\frac{1}{L_{k}} \int \mathbf{E} d t .
$$

Эквивалентная схема такой линии, заполненной диссипативной плазмой, представлена на рис. 3 (в).

Рассмотрим решение системы уравнений (7.12) в точке $\omega=\omega_{p}$. При этом

$$
\varepsilon_{0} \frac{\partial \mathbf{E}}{\partial t}+\frac{1}{L_{k}} \int \mathbf{E} d t=0
$$

поэтому получаем

$$
\operatorname{rot} \mathbf{E}=-\mu_{0} \frac{\partial \mathbf{H}}{\partial t}, \quad \operatorname{rot} \mathbf{H}=\frac{1}{Q_{P}} \sqrt{\frac{\varepsilon_{0}}{L_{k}}} \mathbf{E} .
$$

Эти соотношения и определяют волновые процессы в точке резонанса.

Если потери в плазме, заполняющей линию, малы, а к линии подключен сторонний источник тока, то можно положить:

$$
\operatorname{rot} \mathbf{E} \cong 0, \frac{1}{Q_{p}} \sqrt{\frac{\varepsilon_{0}}{L_{k}}} \mathbf{E}+\varepsilon_{0} \frac{\partial \mathbf{E}}{\partial t}+\frac{1}{L_{k}} \int \mathbf{E} d t=\mathbf{j}_{C T},
$$

где $\mathbf{j}_{\text {ст }}$ - плотность сторонних токов.

Интегрируя (7.13) по времени и разделив обе части на $\varepsilon_{0}$, получим:

$$
\omega_{p}^{2} \mathbf{E}+\frac{\omega_{p}}{Q_{p}} \cdot \frac{\partial \mathbf{E}}{\partial t}+\frac{\partial^{2} \mathbf{E}}{\partial t^{2}}=\frac{1}{\varepsilon_{0}} \cdot \frac{\partial \mathbf{j}_{C T}}{\partial t} .
$$

Интегрированием (7.14) по поверхности, нормальной к вектору Е и введением электрического потока $\Phi_{E}=\int \mathbf{E} d \mathbf{s}$ получим:

$$
\omega_{p}^{2} \Phi_{E}+\frac{\omega_{p}}{Q_{p}} \cdot \frac{\partial \Phi_{E}}{\partial t}+\frac{\partial^{2} \Phi_{E}}{\partial t^{2}}=\frac{1}{\varepsilon_{0}} \cdot \frac{\partial I_{C T}}{\partial t}
$$

где $I_{C T}-$ сторонний ток.

Уравнение (7.15) является уравнением гармонического осциллятора с правой частью, характерное для двухуровневых лазеров [28]. Если источ- 
ник возбуждения отключить, то соотношение (7.14) представляет «холодный» лазерный резонатор с экспоненциальным затуханием колебаний вида

$$
\Phi_{E}(t)=\Phi_{E}(0) e^{i \omega_{P} t} \cdot e^{-\frac{\omega_{P}}{2 Q_{P}} t}
$$

т.е. макроскопический электрический поток $\Phi_{E}(t)$ будет осциллировать с частотой $\omega_{p}$. Время релаксации при этом определяется соотношением:

$$
\tau=2 Q_{P} / \omega_{P}
$$

Задача создания лазера состоит лишь в умении возбудить такой резонатор.

Если резонатор возбуждается сторонними токами, то такой резонатор для этих токов представляет полосовой фильтр с резонансной частотой, равной плазменной частоте с полосой пропускания $\Delta \omega=\omega_{p} /\left(2 Q_{p}\right)$.

Другим важным практическим применением поперечного плазменного резонанса является возможность его использования для разогрева и диагностики плазмы. Если добротность плазменного резонатора велика, то могут быть получены высокие уровни электрических полей, а значит и высокие энергии носителей зарядов.

\section{§ 8. Кинетическая ёмкость}

Если учесть все составляющие плотности токов в проводнике, то второе уравнение Максвелла имеет вид:

$$
\operatorname{rot} \mathbf{H}=\sigma_{E} \mathbf{E}+\varepsilon \frac{\partial \mathbf{E}}{\partial t}+\frac{1}{L_{k}} \int \mathbf{E} d t
$$

где $\sigma_{E}$ - проводимость металла.

В то же время, первое уравнение Максвелла можно записать так:

$$
\operatorname{rot} \mathbf{E}=-\mu \frac{\partial \mathbf{H}}{\partial t},
$$

где $\mu$ - магнитная проницаемость среды. Видно, что уравнения (8.1) и (8.2) несимметричны.

Несколько улучшить симметрию этих уравнений можно, вводя в уравнение (8.2) член, линейный по магнитному полю, учитывающий тепловые потери в магнетиках в переменных полях:

$$
\operatorname{rot} \mathbf{E}=-\sigma_{H} \mathbf{H}-\mu \frac{\partial \mathbf{H}}{\partial t},
$$

где $\sigma_{H}$ - проводимость магнитных токов. Но вот интеграла такого типа, который имеется в правой части уравнения (8.1), в данном уравнении нет. В то же время нам известно, что атом, обладающий магнитным моментом $\mathbf{m}$, помещённый в магнитное поле, и осуществляющий в нём прецессионное движение, имеет потенциальную энергию $U_{m}=-\boldsymbol{\mu} \mathbf{m H}$. Поэтому потенциальная энергия может накапливаться не только в электрических полях, но и в прецессионном движении магнитных моментов, которое не обладает инерцией. Аналогичный случай имеется и в механике, когда гиро- 
скоп, прецессирующий в поле внешних сил, накапливает потенциальную энергию. По определению механическое прецессионное движение также является безынерционным и сразу же прекращается после снятия внешних сил. Например, если из-под прецессирующего волчка, вращающегося в поле земного тяготения, быстро убрать опору, то он начнёт падать, сохраняя в пространстве то направление своей оси, которое было в момент, когда была убрана опора. Такая же ситуация имеет место и для случая прецессирующего магнитного момента. Его прецессия является безынерционной и прекращается в момент снятия магнитного поля.

С учётом сказанного можно ожидать, что при описании прецессионного движения магнитного момента во внешнем магнитном поле в правой части соотношения (8.3) может появиться слагаемое того же типа, что и в соотношении (8.1). Только вместо $L_{k}$ будет стоять $C_{k}$, т.е. кинетическая ёмкость, характеризующая ту потенциальную энергию, которую имеет прецессирующий магнитный момент в магнитном поле $[9,29,30]$ :

$$
\operatorname{rot} \mathbf{E}=-\sigma_{H} \mathbf{H}-\mu \frac{\partial \mathbf{H}}{\partial t}-\left(1 / C_{k}\right) \int \mathbf{H} d t .
$$

Впервые такое представление первого уравнения Максвелла с учётом кинетической ёмкости было дано в работе [10].

Может ли реализоваться такой случай на практике, и что такое кинетическая ёмкость? Резонансные процессы в плазме и диэлектриках характеризуются попеременным преобразованием в процессе колебаний электростатической энергии в кинетическую энергию движения зарядов и наоборот. Такой процесс может быть назван электрокинетическим, и все устройства (лазеры, мазеры, фильтры и т.д.), использующие этот процесс, могут быть названы электрокинетическими. Наряду с этим существует другой тип резонанса - магнитный. Пользуясь существующими представлениями о зависимости магнитной проницаемости от частоты, нетрудно показать, что такая зависимость связана с наличием магнитного резонанса. Для этого рассмотрим конкретный пример ферромагнитного резонанса. Если намагнитить феррит, приложив постоянное поле $H_{0}$ параллельно оси $z$, то по отношению к внешнему переменному полю среда служит анизотропным магнетиком с комплексной проницаемостью в виде тензора [31]

$$
\mu=\left(\begin{array}{lll}
\mu_{T} *(\omega) & -i \alpha & 0 \\
i \alpha & \mu_{T}^{*}(\omega) & 0 \\
0 & 0 & \mu_{L}
\end{array}\right),
$$

где

$$
\mu_{T} *(\omega)=1-\frac{\Omega|\gamma| M_{0}}{\mu_{0}\left(\omega^{2}-\Omega^{2}\right)}, \quad \alpha=\frac{\omega|\gamma| M_{0}}{\mu_{0}\left(\omega^{2}-\Omega^{2}\right)}, \quad \mu_{L}=1,
$$

причем

$$
\Omega=\gamma H_{0}
$$

есть собственная частота прецессии, а 


$$
M_{0}=\mu_{0}(\mu-1) H_{0}
$$

есть намагниченность среды. Учитывая (8.4) и (8.5) для $\mu_{T}^{*}(\omega)$, имеем:

$$
\mu_{T}^{*}(\omega)=1-\frac{\Omega^{2}(\mu-1)}{\omega^{2}-\Omega^{2}} .
$$

Получилось, что магнитная проницаемость магнетика зависит от частоты, и легко предположить, что данный случай аналогичен случаю плазмы.

Считая, что электромагнитная волна распространяется по оси $x$ и имеются компоненты полей $H_{y}$ и $H_{z}$, запишем первое уравнение Максвелла:

$$
\operatorname{rot} \mathbf{E}=\frac{\partial \mathbf{E}_{Z}}{\partial x}=\mu_{0} \mu_{T} \frac{\partial \mathbf{H}_{y}}{\partial t} .
$$

Учитывая (8.6), получим:

$$
\operatorname{rot} \mathbf{E}=\mu_{0}\left[1-\frac{\Omega^{2}(\mu-1)}{\omega^{2}-\Omega^{2}}\right] \frac{\partial \mathbf{H}_{y}}{\partial t} .
$$

Для случая $\omega>>\Omega$ имеем:

$$
\operatorname{rot} \mathbf{E}=\mu_{0}\left[1-\frac{\Omega^{2}(\mu-1)}{\omega^{2}}\right] \frac{\partial \mathbf{H}_{y}}{\partial t} .
$$

Полагая $H_{y}=H_{y 0} \sin \omega t$ и учитывая, что в этом случае

из (8.7) получим

$$
\frac{\partial \mathbf{H}_{y}}{\partial t}=-\omega^{2} \int \mathbf{H}_{y} d t
$$

$$
\operatorname{rot} \mathbf{E}=\mu_{0} \frac{\partial \mathbf{H}_{y}}{\partial t}+\mu_{0} \Omega^{2}(\mu-1) \int \mathbf{H}_{y} d t
$$

или

$$
\operatorname{rot} \mathbf{E}=\mu_{0} \frac{\partial \mathbf{H}_{y}}{\partial t}+\frac{1}{C_{k}} \int \mathbf{H}_{y} d t .
$$

Для случая $\omega<<\Omega$ находим

Величину

$$
\operatorname{rot} \mathbf{E}=\mu_{0} \mu \frac{\partial \mathbf{H}_{y}}{\partial t} .
$$

$$
C_{k}=\frac{1}{\mu_{0} \Omega^{2}(\mu-1)},
$$

которая введена в соотношении (8.8), назовем кинетической емкостью.

Каков ее физический смысл? Если направления магнитного момента и внешнего магнитного поля не совпадают, то вектор момента прецессирует вокруг вектора магнитного поля с частотой $\Omega$. Магнитный момент $\mathbf{m}$ обладает при этом энергией $U_{m}=-\mathbf{m} \cdot \mathbf{B}$, потенциальной подобно энергии заряженного конденсатора, потому что прецессионное движение не инерционно и мгновенно прекращается при снятии магнитного поля. При наличии же магнитного поля прецессионное движение продолжается до тех пор, 
пока не будет израсходована накопленная потенциальная энергия, и вектор магнитного момента не станет параллельным вектору магнитного поля.

Эквивалентная схема этого случая приведена на рис. 4. В точке $\omega=\Omega$ есть магнитный резонанс, при этом $\mu_{\mathrm{T}}^{*}(\omega) \rightarrow-\infty$. Резонансная частота макроскопического магнитного резонатора, как видно из схемы, также не зависит от размеров линии и равна $\Omega$. Таким образом, параметр

$$
\mu_{H} *(\omega)=\mu_{0}\left[1-\Omega^{2}(\mu-1) /\left(\omega^{2}-\Omega^{2}\right)\right]
$$

не является частотно зависимой магнитной проницаемостью, а включает в себя $\mu_{0}, \mu$ и $C_{k}$, которые включены в соответствии со схемой на рис. 4.

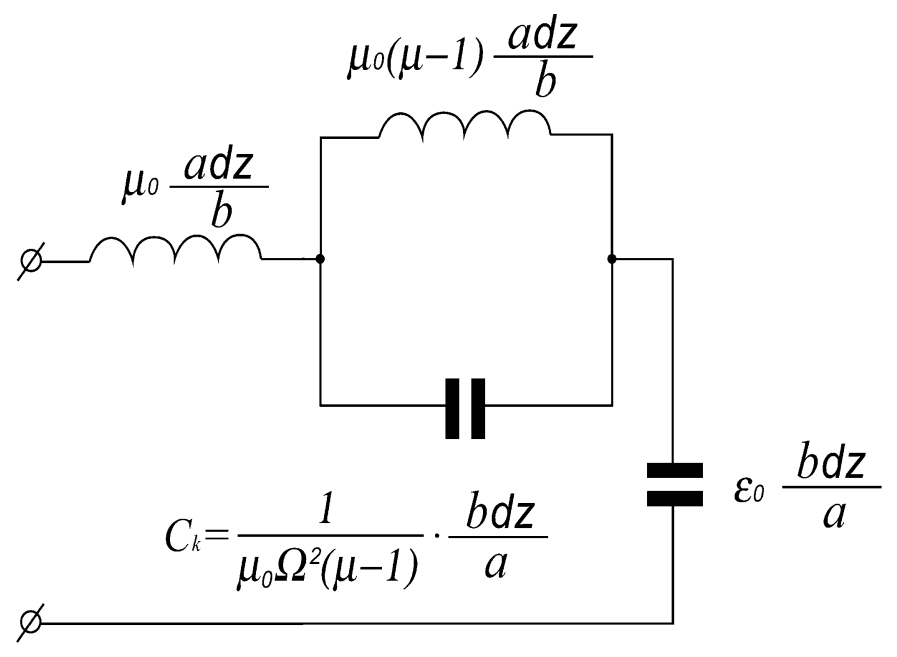

Рис. 4. Эквивалентная схема двухпроводной линии, заполненной магнетиком

Нетрудно показать, что в данном случае имеет место распространение трех волн: электрической, магнитной и волны, несущей потенциальную энергию, которая связана с прецессией магнитных моментов вокруг вектоpa $H_{0}$. По этой причине такие волны могут быть названы электромагнитнопотенциальными. До появления работы [9] в электродинамике такое понятие, как кинетическая ёмкость не использовалось, хотя это реальный параметр имеет очень понятную физическую интерпретацию.

\section{§ 9. Диэлектрики}

В научной литературе не раскрыта роль кинетической индуктивности носителей зарядов в электродинамических процессах в диэлектриках. Но этот параметр в электродинамике диэлектриков играет не менее важную роль, чем в электродинамике проводников [32,33]. В простейшем случае, когда колебательные процессы в атомах или молекулах диэлектрика подчиняются законам механического осциллятора, запишем уравнение движения: 


$$
\left(\frac{\beta}{m}-\omega^{2}\right) \mathbf{r}_{m}=\frac{e}{m} \mathbf{E},
$$

где $\mathbf{r}_{m}$ - отклонение зарядов от положения равновесия, а $\beta$ - коэффициент упругости, характеризующий упругость электрических сил связи зарядов в атомах и молекулах. Вводя резонансную частоту связанных зарядов

$$
\omega_{0}=\beta / m,
$$

из (9.1) получаем

$$
\mathbf{r}_{m}=-\frac{e \mathbf{E}}{m\left(\omega^{2}-\omega_{o}^{2}\right)} .
$$

Видно, что в соотношении (9.2) в качестве параметра присутствует частота собственных колебаний, в которую входит масса заряда. Это говорит о том, что инерционные свойства колеблющихся зарядов будут влиять на колебательные процессы в атомах и молекулах.

Поскольку общая плотность тока в среде состоит из тока смещения и тока проводимости

$$
\operatorname{rot} \mathbf{H}=\mathbf{j}_{\Sigma}=\varepsilon_{0} \frac{\partial \mathbf{E}}{\partial t}+n e \mathbf{v},
$$

то, находя скорость носителей зарядов в диэлектрике как производную их смещения по координате

$$
\mathbf{v}=\frac{\partial \mathbf{r}_{m}}{\partial t}=-\frac{e}{m\left(\omega^{2}-\omega_{o}^{2}\right)} \frac{\partial \mathbf{E}}{\partial t}
$$

из соотношения (9.2) находим

$$
\operatorname{rot} \mathbf{H}=\mathbf{j}_{\Sigma}=\varepsilon_{0} \frac{\partial \mathbf{E}}{\partial t}-\frac{1}{L_{k d}\left(\omega^{2}-\omega_{0}^{2}\right)} \frac{\partial \mathbf{E}}{\partial t} .
$$

Заметим, что величина

$$
L_{k d}=m /\left(n e^{2}\right)
$$

есть кинетическая индуктивность зарядов в составе атомов или молекул диэлектриков, считая их свободными. Поэтому (9.3) перепишем в виде:

$$
\operatorname{rot} \mathbf{H}=\mathbf{j}_{\Sigma}=\varepsilon_{0}\left(1-\frac{1}{\varepsilon_{0} L_{k d}\left(\omega^{2}-\omega_{0}{ }^{2}\right)}\right) \frac{\partial \mathbf{E}}{\partial t} .
$$

Так как величина

$$
1 /\left(\varepsilon_{0} L_{k d}\right)=\omega_{p d}^{2}
$$

представляет плазменную частоту зарядов в атомах и молекулах диэлектрика, если считать эти заряды свободными, то (9.4) принимает вид:

$$
\operatorname{rot} \mathbf{H}=\mathbf{j}_{\Sigma}=\varepsilon_{0}\left(1-\frac{\omega_{p d}^{2}}{\left(\omega^{2}-\omega_{0}^{2}\right)}\right) \frac{\partial \mathbf{E}}{\partial t} .
$$

И опять можно назвать величину

$$
\varepsilon^{*}(\omega)=\varepsilon_{0}\left(1-\frac{\omega_{p d}^{2}}{\left(\omega^{2}-\omega_{0}^{2}\right)}\right)
$$


эффективной диэлектрической проницаемостью диэлектрика. Она опятьтаки зависит от частоты. Но данный математический параметр не является физической диэлектрической проницаемостью диэлектрика, а имеет сборный характер. Он включает в себя теперь уже три не зависящих от частоты величины: электрическую постоянную, собственную частоту атомов или молекул и плазменную частоту для носителей зарядов, входящих в их состав, если считать заряды свободными.

Рассмотрим два предельных случая:

1. Если $\omega<<\omega_{0}$, то из (9.5) получаем

$$
\operatorname{rot} \mathbf{H}=\mathbf{j}_{\Sigma}=\varepsilon_{0}\left(1+\frac{\omega_{p d}{ }^{2}}{{\omega_{0}}^{2}}\right) \frac{\partial \mathbf{E}}{\partial t} .
$$

В этом случае коэффициент перед производной не зависит от частоты, и представляет собой статическую абсолютную диэлектрическую проницаемость диэлектрика. Она зависит от собственной частоты колебаний атомов или молекул и от плазменной частоты. Этот результат понятен. Частота в данном случае оказывается настолько низкой, что заряды успевают следовать за полем и их инерционные свойства на электродинамические процессы не влияют. В этом случае выражение в скобках в правой части соотношения (9.7) представляет собой статическую относительную диэлектрическую проницаемость диэлектрика. Она зависит от собственной частоты колебаний самих атомов или молекул диэлектрика и от плазменной частоты. Отсюда сразу имеем рецепт для создания диэлектриков с высокой диэлектрической проницаемостью. Чтобы достичь этого, следует в заданном объёме пространства упаковать максимальное количество молекул с максимально мягкими связями между зарядами внутри самой молекулы.

2. Показательным является случай $\omega>>\omega_{0}$. При этом

$$
\operatorname{rot} \mathbf{H}=\mathbf{j}_{\Sigma}=\varepsilon_{0}\left(1-\frac{\omega_{p d}^{2}}{\omega^{2}}\right) \frac{\partial \mathbf{E}}{\partial t}
$$

и диэлектрик превратился в проводник (плазму), т.к. полученное соотношение совпадает с уравнением, описывающим плазму.

Нельзя не заметить то обстоятельство, что в данном случае опять нигде не использовалось такое понятие как вектор поляризации, а рассмотрение проведено путём нахождения реальных токов в диэлектриках на основе уравнения движения зарядов в этих средах. При этом в данной математической модели в качестве исходных электрических характеристик среды использованы величины, которые от частоты не зависят.

Из соотношения (9.5) видно, что в случае выполнения равенства $\omega=\omega_{0}$ амплитуда колебаний равна бесконечности. Это означает наличие резонанса в этой точке. Бесконечная амплитуда колебаний имеет место по причине того, что не учитывались потери в резонансной системе, при этом её добротность равна бесконечности. В каком-то приближении можно считать, что ниже указанной точки мы имеем дело с диэлектриком, у которого диэлектрическая проницаемость равна её статическому значению. Выше этой 
точки мы имеем дело уже фактически с металлом, у которого плотность носителей тока равна плотности атомов или молекул в диэлектрике.

Рассмотрим с электродинамической точки зрения разложение диэлектрической призмой полихроматического света на монохроматические составляющие или почему образуется радуга. Для этого фазовая скорость электромагнитных волн в среде должна зависеть от частоты (частотная дисперсия волн). Добавим к (9.5) первое уравнение Максвелла:

$$
\operatorname{rot} \mathbf{E}=-\mu_{0} \frac{\partial \mathbf{H}}{\partial t} ; \quad \operatorname{rot} \mathbf{H}=\varepsilon_{0}\left(1-\frac{\omega_{p d}^{2}}{\left(\omega^{2}-\omega_{0}^{2}\right)}\right) \frac{\partial \mathbf{E}}{\partial t},
$$

откуда сразу находим волновое уравнение:

$$
\nabla^{2} \mathbf{E}=\mu_{0} \varepsilon_{0}\left(1-\frac{\omega_{p d}{ }^{2}}{\omega^{2}-\omega_{0}^{2}}\right) \frac{\partial^{2} \mathbf{E}}{\partial t^{2}}
$$

Если учесть, что

$$
\mu_{0} \varepsilon_{0}=1 / c^{2},
$$

где $c$ - скорость света, то легко видеть наличие в диэлектриках частотной дисперсии. Но зависимость фазовой скорости от частоты связана не с зависимостью от нее физической диэлектрической проницаемости. В формировании дисперсии участвуют три не зависящие от частоты физические величины: собственная резонансная частота атомов или молекул, плазменная частота зарядов, считая их свободными, и электрическая постоянная.

Теперь покажем слабые места традиционного подхода, основанного на использовании понятия вектора поляризации

$$
\mathbf{P}=-\frac{n e^{2}}{m} \cdot \frac{1}{\left(\omega^{2}-\omega_{0}^{2}\right)} \mathbf{E} .
$$

Его зависимость от частоты связана с наличием массы у зарядов, входящих в состав атомов и молекул диэлектриков. Инерционность зарядов не позволяет этому вектору, следуя за электрическим полем, достигать того значения, которое он имел бы в статических полях. Поскольку электрическая индукция определяется соотношением

$$
\mathbf{D}=\varepsilon_{0} \mathbf{E}+\mathbf{P E}=\varepsilon_{0} \mathbf{E}-\frac{n e^{2}}{m} \cdot \frac{1}{\left(\omega^{2}-\omega_{0}^{2}\right)} \mathbf{E},
$$

то, введённая таким способом, она зависит от частоты.

Введя такую индукцию во второе уравнение Максвелла, получим:

$$
\operatorname{rot} \mathbf{H}=\mathbf{j}_{\Sigma}=\varepsilon_{0} \frac{\partial \mathbf{E}}{\partial t}+\frac{\partial \mathbf{P}}{\partial t}
$$

или

$$
\operatorname{rot} \mathbf{H}=\mathbf{j}_{\Sigma}=\varepsilon_{0} \frac{\partial \mathbf{E}}{\partial t}-\frac{n e^{2}}{m} \frac{1}{\left(\omega^{2}-\omega_{0}^{2}\right)} \frac{\partial \mathbf{E}}{\partial t},
$$

где $\mathbf{j}_{\Sigma}$ - суммарный ток через образец. В (9.9) первый член правой части ток смещения в вакууме, а второй - ток, связанный с наличием связанных 
зарядов в атомах или молекулах. Опять появилась удельная кинетическая индуктивность участвующих в колебательном процессе зарядов

$$
L_{k d}=m / n e^{2},
$$

определяющая индуктивность связанных зарядов, и (9.9) принимает вид:

$$
\operatorname{rot} \mathbf{H}=\mathbf{j}_{\Sigma}=\varepsilon_{0} \frac{\partial \mathbf{E}}{\partial t}-\frac{1}{L_{k d}} \frac{1}{\left(\omega^{2}-\omega_{0}^{2}\right)} \frac{\partial \mathbf{E}}{\partial t} .
$$

Полученное выражение в точности совпадает с соотношением (9.3). Так как конечный результат рассмотрения обоими способами совпадает, то с математической точки зрения претензий к методу нет. Но с физической точки зрения, и особенно в части присвоения параметру, введённому в соответствии с соотношением (9.8), наименования электрической индукции, имеются большие претензии, которые мы уже обсудили. Это не физическая величина электрической индукции, а некий сборный математический параметр. По сути, введение электрической индукции в диэлектриках физически обоснованно только для статических электрических полей.

Покажем, что эквивалентная схема диэлектрика в данном случае представляет последовательный резонансный контур с индуктивностью, равной кинетическая индуктивность $L_{k d}$, и ёмкостью, равной статической диэлектрической проницаемости диэлектрика за вычетом ёмкости, равной диэлектрической проницаемости вакуума. Контур зашунтирован ёмкостью, равной удельной диэлектрической проницаемости вакуума. Для доказательства этого рассмотрим последовательный колебательный контур, когда индуктивность $L$ и ёмкость $C$ включены последовательно.

Связь между током $I_{C}$, текущим через ёмкость $C$, и напряжением $U_{C}$, приложенным к ней, определяется соотношениями:

$$
\begin{gathered}
U_{C}=\frac{1}{C} \int I_{C} d t ; \\
I_{C}=C \frac{d U_{C}}{d t} .
\end{gathered}
$$

Для индуктивности эта связь запишется:

$$
I_{L}=\frac{1}{L} \int U_{L} d t ; U_{L}=L \frac{d I_{L}}{d t} .
$$

Если ток через последовательный контур меняется по закону $I=I_{0} \sin \omega t$, то падение напряжения на индуктивности и ёмкости соответственно равно

$$
U_{L}=\omega L I_{0} \cos \omega t ; U_{C}=-\frac{1}{\omega C} I_{0} \cos \omega t,
$$

а суммарное приложенное напряжение будет равно

$$
U_{\Sigma}=(\omega L-1 /(\omega C)) I_{0} \cos \omega t .
$$

В этом соотношении величина, стоящая в скобках, представляет реактивное сопротивление последовательного резонансного контура, которое зависит от частоты. Напряжения, генерируемые на ёмкости и индуктивности, находятся в противофазе, и, в зависимости от частоты, контур может иметь 
то ли индуктивное, то ли ёмкостное реактивное сопротивление. В точке резонанса суммарное реактивное сопротивление контура равно нулю.

Очевидно, что связь между суммарным приложенным напряжением и током, текущим через контур, будет определяться соотношением

$$
I=-\frac{1}{\omega(\omega L-1 /(\omega C))} \frac{\partial U_{\Sigma}}{\partial t} .
$$

Резонансная частота контура определяется соотношением

$$
\omega_{0}=1 / \sqrt{L C},
$$

поэтому запишем

$$
I=\frac{C}{\left(1-\omega^{2} / \omega_{0}^{2}\right)} \frac{\partial U_{\Sigma}}{\partial t} .
$$

Сравнивая (9.12) с (9.10), нетрудно видеть, что последовательный резонансный контур, состоящий из индуктивности $L$ и ёмкости $C$, можно представить в виде эффективной ёмкости, зависящей от частоты:

$$
C(\omega)=C /\left(1-\omega^{2} / \omega_{0}^{2}\right) .
$$

При таком представлении индуктивность не теряется, так как входит в резонансную частоту контура $\omega_{0}$. Соотношения (9.12) и (9.11) эквивалентны. Следовательно, величина $C(\omega)$ не является физической величиной ёмкости контура, представляя собой некий сборный математический параметр.

Соотношение (9.11) можно переписать и по-другому:

$$
I=-\frac{1}{L\left(\omega^{2}-\omega_{0}^{2}\right)} \frac{\partial U_{\Sigma}}{\partial t}
$$

и считать, что

$$
C(\omega)=-\frac{1}{L\left(\omega^{2}-\omega_{0}^{2}\right)} .
$$

Конечно, параметр $C(\omega)$, введённый в соответствии с соотношениями (9.13) и (9.14), никакого отношения к ёмкости не имеет.

Рассмотрим соотношение (9.12) для двух предельных случаев:

1. Когда $\omega<<\omega_{0}$, имеем

$$
I=C \frac{\partial U_{\Sigma}}{\partial t} .
$$

Этот результат понятен, т.к. на низких частотах реактивное сопротивление индуктивности, включённой последовательно с ёмкостью, значительно меньше ёмкостного, и его можно не учитывать.

Эквивалентная схема диэлектрика, расположенного между плоскостями длинной линии, показана на рис. 5.

2. Когда $\omega>>\omega_{0}$, имеем

$$
I=-\frac{1}{\omega^{2} L} \frac{\partial U_{\Sigma}}{\partial t} .
$$

Учитывая, что для гармонического сигнала 
из (9.15) получаем

$$
\frac{\partial U_{\Sigma}}{\partial t}=-\omega^{2} \int U_{\Sigma} d t,
$$

$$
I_{L}=\frac{1}{L} \int U_{\Sigma} d t
$$

В данном случае реактивное сопротивление ёмкости значительно меньше, чем у индуктивности, и цепь имеет индуктивное сопротивление.

На практике трудно отличить поведение резонансных контуров от чистой индуктивности или ёмкости, особенно вдали от резонанса, где отличия практически отсутствуют. Для понимания истинного состава цепи нужно снять ее амплитудную и фазовую характеристику в диапазоне частот. В случае резонансного контура такая зависимость будет иметь типичный резонансный характер, когда по обе стороны резонанса характер реактивного сопротивления будет разным. Однако это не означает, что реальные элементы контура (ёмкость или индуктивность) зависят от частоты.

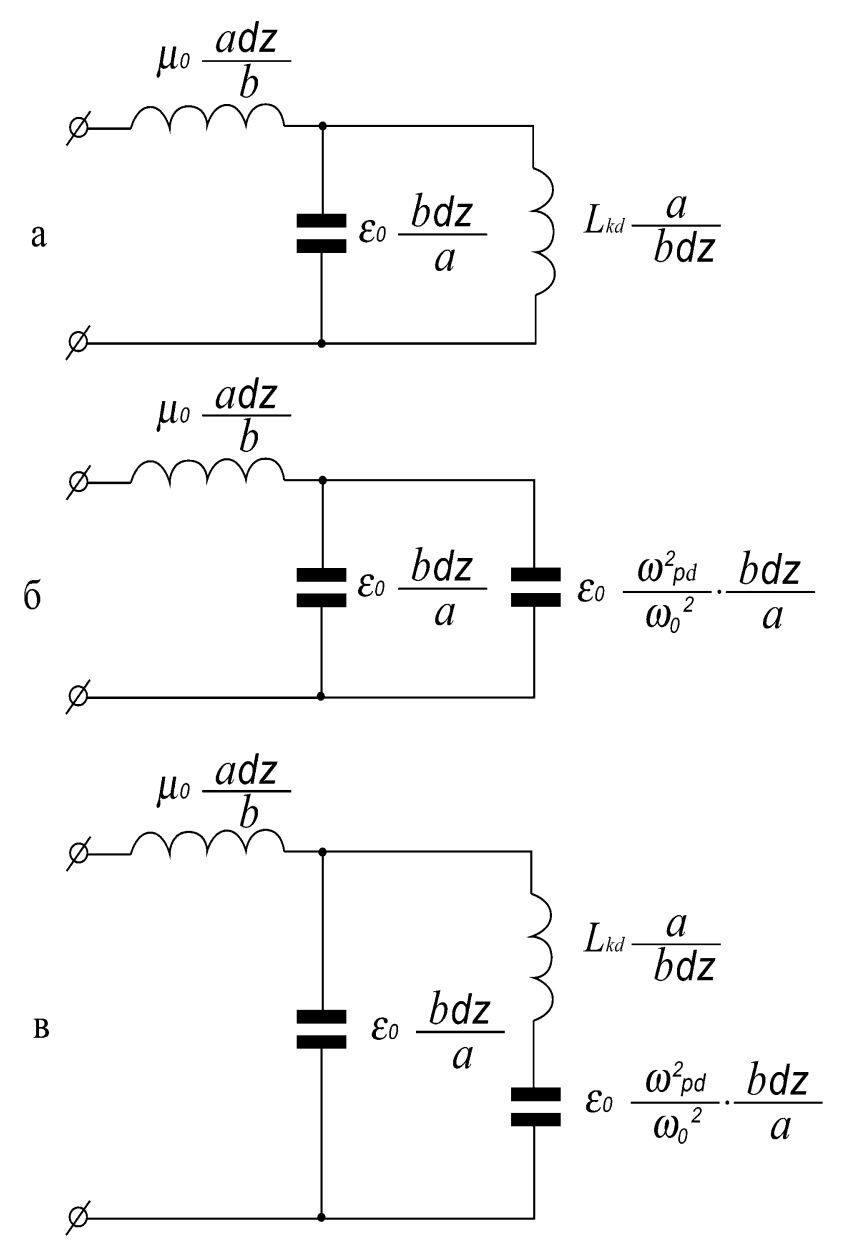

Рис. 5. а - эквивалентная схема отрезка линии, заполненной диэлектриком, для случая $\omega>>\omega_{0}$;

б - эквивалентная схема отрезка линии для случая $\omega<<\omega_{0}$;

в - эквивалентная схема отрезка линии для всего диапазона частот

На рис. 5а и $5 б$ показаны два предельных случая: $\omega>>\omega_{0}$, когда свойства 
диэлектрика соответствуют проводнику; $\omega<<\omega_{0}$, когда - диэлектрику со статической диэлектрической проницаемостью $\varepsilon=\varepsilon_{0}\left(1+\omega_{p d}{ }^{2} / \omega_{0}{ }^{2}\right)$.

Итак, использование термина «диэлектрическая проницаемость диэлектриков» в контексте ее зависимости от частоты не вполне корректно. Если речь идёт о диэлектрической проницаемости диэлектриков, с которой связано накопление потенциальной энергии, то корректно рассматривать только статическую проницаемость, являющуюся постоянной величиной, не зависящей от частоты. Именно она входит во все соотношения, характеризующие электродинамические характеристики диэлектриков.

Применение таких новых подходов наиболее интересно именно для диэлектриков. Тогда каждая связанная пара зарядов есть отдельная унитарная единица со своими индивидуальными характеристиками, и её взаимодействие с электромагнитным полем (без учета связей между парами) строго индивидуально. Конечно, не все диполи имеют разные характеристики, а имеются различные группы с подобными характеристиками, и каждая группа связанных зарядов с одинаковыми характеристиками резонирует на своей частоте. Интенсивность поглощения, а в возбужденном состоянии и излучения, на этой частоте зависит от относительного количества пар данного сорта. Поэтому можно ввести соответствующие парциальные коэффициенты. На эти процессы влияет анизотропия диэлектрических свойств молекул, имеющих определенную электрическую ориентацию в кристаллической решетке. Этими обстоятельствами определяется многообразие резонансов и их интенсивностей в диэлектрических средах. При электрической связи между отдельными группами излучателей линии поглощения или излучения могут превращаться в полосы. Такой индивидуальный подход к сортам связанных пар зарядов невозможен в имевшихся теориях.

Подчеркнем важное обстоятельство, не получившее пока должной оценки. Во всех соотношениях для любых материальных сред (проводники и диэлектрики) наряду с диэлектрической и магнитной проницаемостью фигурирует кинетическая индуктивность зарядов, что говорит о не менее важной роли этого параметра. Это впервые отмечено в ряде упомянутых источников, в том числе в недавно опубликованной статье [23]. 


\section{ГЛАВА З. НОВЫЕ МЕТОДИКИ И ПОНЯТИЯ}

\section{§ 10. Поверхностная кинетическая индуктивность}

До сих пор считалось, что кинетическая индуктивность эффективно проявляется только в сверхпроводниках, и вводилась она феноменологическим способом. Но для проводящих сред кроме объёмной кинетической индуктивности можно ввести поверхностную кинетическую индуктивность, расширив таким образом границы применимости данного термина.

Часть энергии падающей на границу материальной среды плоской электромагнитной волны поглощается, а часть - отражается. Рассмотрим случай, когда частота падающей волны значительно ниже плазменной [8].

Уравнения Максвелла для комплексных амплитуд полей примут вид:

$$
\operatorname{rot} \mathbf{E}=-i \omega \mu_{0} \mathbf{H} ; \quad \operatorname{rot} \mathbf{H}=\mathbf{j} ; \quad \operatorname{div} \mathbf{E}=0 ; \quad \operatorname{div} \mathbf{H}=0 .
$$

Здесь и далее закон изменения электромагнитного поля взят в виде $e^{i \omega t}$.

Поверхностное сопротивление $R$ и поверхностный реактанс $X$ связывают тангенциальные составляющие электрического и магнитного поля на поверхности и определяют энергетические характеристики взаимодействия поверхности с электромагнитным полем. Комплексные амплитуды тангенциальных составляющих полей на поверхности связаны соотношением

$$
E_{T}=Z H_{T},
$$

из которого получим связь между реальными полями на поверхности

$$
\left|\tilde{E}_{T}\right|=\left|Z \| \tilde{H}_{T}\right| \cos (\omega t+\varphi),
$$

где поверхностный импеданс поверхности равен

$$
Z=\frac{E_{x}(0)}{H_{y}(0)}=i \omega \mu_{0} \frac{1}{H_{y}(0)} \int_{0}^{\infty} H_{y}(z) d z .
$$

Его модуль - отношение амплитуд тангенциальных составляющих электрического и магнитного полей на поверхности, а фаза - сдвиг фаз между ними. Для установления связи $R$ и $X$ с энергетическими характеристиками поверхностного слоя возьмём единичный участок поверхности, для которого справедливы граничные условия Леонтовича. Умножим первое уравнение (10.1) на $\mathbf{H}^{*}$, второе - на $\mathbf{E}$ и почленно вычтем одно из другого:

$$
\operatorname{div} \mathbf{P}=-\frac{1}{2} \mathbf{j}^{*} \mathbf{E}-i \omega \frac{\mu_{0}|\mathbf{H}|^{2}}{2},
$$

где $\mathbf{P}=\frac{1}{2}\left[\mathbf{E} \times \mathbf{H}^{*}\right]$ - комплексный вектор Пойнтинга. Интегрируя (10.2) по объёму под единичной площадкой, применим формулу Гаусса:

$$
\int_{S} \mathbf{P} d \mathbf{S}=-\frac{1}{2} \int_{V} \mathbf{j}^{*} \mathbf{E} d S d z-i 2 \omega \int_{V} \frac{\mu_{0}|\mathbf{H}|^{2}}{4} d S d z
$$

где интегрирование ведется по поверхности выделенной площадки, а элемент объёма записан в виде $d S d z$. 
Положим, что поля мало меняются в пределах выделенной площадки в тангенциальном направлении и обращаются в нуль при $z \rightarrow \infty$.

В поверхностном интеграле в (10.3) принято $\mathbf{P} d \mathbf{S}=-\mathbf{P} \mathbf{n} d S=-P_{n} d S$, где вектор $\mathbf{n}$ направлен вглубь рассматриваемой среды. В соотношении (10.3) существенны только тангенциальные компоненты $\mathbf{E}$ и $\mathbf{H}$. Учитывая, что

$$
\left[\mathbf{E}_{T} \times \mathbf{H}_{T}{ }^{*}\right]=Z\left|\mathbf{H}_{T}\right|^{2} \vec{n},
$$

это уравнение приводится к виду:

$$
\frac{1}{2} Z\left|\mathbf{H}_{T}(0)\right|^{2}=\frac{1}{2} \int_{0}^{\infty} \mathbf{j}^{*} \mathbf{E} d z+i 2 \omega \int_{0}^{\infty} \frac{\mu_{0}|\mathbf{H}|^{2}}{4} d z .
$$

Выделив действительную часть этого равенства, получим:

$$
P_{R}=\frac{1}{2} R\left|H_{T}(0)\right|^{2}=\operatorname{Re} \frac{1}{2} \int_{0}^{\infty} \mathbf{j}^{*} \mathbf{E} d z,
$$

где $P_{R}$ - средняя мощность потерь на единичный квадрат поверхности.

Выделяя мнимую часть уравнения (10.4), находим:

$$
P_{X}=\frac{1}{2} X\left|\mathbf{H}_{T}(0)\right|^{2}=\operatorname{Im} \frac{1}{2} \int_{0}^{\infty} \mathbf{j}^{*} \mathbf{E} d z+2 \omega \int_{0}^{\infty} \frac{\mu_{0}|\mathbf{H}|^{2}}{4} d z,
$$

где $P_{X}$ - средняя реактивная мощность на единичный квадрат поверхности.

Она состоит из двух членов: реактивной мощности, связанной с кинетической энергией носителей тока, и связанной с магнитным полем.

Граничные условия

$$
\mathbf{E}_{T}(0)=Z\left[\mathbf{H}_{T}(0) \times \mathbf{n}\right], Z=R+i X
$$

для действительных величин полей $\tilde{E}_{T}$ и $\tilde{H}_{T}$ можно записать в виде

$$
\tilde{E}_{T}=R \tilde{H}_{T}+L \frac{d \tilde{H}}{d t},
$$

где $L=X / \omega$ - поверхностная индуктивность поверхности.

Теперь можно ввести ещё такие новые понятия, как кинетическую и полевую поверхностную индуктивности для случая произвольной связи между током и полем как в нормальных металлах, так и в сверхпроводниках:

$$
L_{K}=\frac{1}{\omega\left|\mathbf{H}_{T}(0)\right|^{2}} \operatorname{Im} \int_{0}^{\infty} \mathbf{j}^{*} \mathbf{E} d z, L_{\Pi}=\frac{1}{\left|\mathbf{H}_{T}(0)\right|^{2}} \int_{0}^{\infty}\left|\mathbf{H}_{T}\right|^{2} d z .
$$

Такой анализ выявил интересный факт. В среде со свободными зарядами могут быть лишь коллективные колебания равноправных зарядов, находящихся в одном и том же энергетическом состоянии с одинаковой суммой кинетической и потенциальной энергии в любой момент времени (без учета потерь), что справедливо для сверхпроводников и холодной плазмы.

\section{§ 11. Электроёмкостная самоиндукция}

К законам самоиндукции следует отнести те законы, которые описывают реакцию таких элементов радиотехнических цепей, как ёмкость, индуктивность и сопротивление при гальваническом подключении к ним источ- 
ников тока или напряжения. Эти законы являются основой теории электрических цепей. Результаты этой теории могут быть перенесены и на электродинамику материальных сред, т.к. такие среды могут быть представлены в виде эквивалентных схем с использованием таких элементов.

Движение зарядов в какой-либо цепи, которые заставляют их менять своё местоположение или двигаться, связано с потреблением энергии от источников питания. Процессы взаимодействия источников питания с такими структурами регулируются законами самоиндукции.

Самоиндукция есть реакция материальных структур с неизменными параметрами на подключение источников напряжения или тока. Самоиндукцию назовем параметрической, если при подключении источника питания или накоплении в системе энергии могут меняться ее параметры. Пусть на любой нагрузке идеальный генератор напряжения обеспечивает заданное напряжение (его внутреннее сопротивление равно нулю), а идеальный генератор тока - заданный ток (его внутреннее сопротивление равно бесконечности). Но реальный генератор имеет внутреннее сопротивление.

Если к элементу цепи подключить генератор тока или напряжения, то ответной реакцией такого элемента является противодействие изменению своего начального состояния, и это противодействие всегда равно приложенному действию, что соответствует третьему закону Ньютона.

Накопленный в емкости $C$ при разности потенциалов $U$ заряд

$$
Q_{C, U}=C U,
$$

зависящий от величины ёмкости и разности потенциалов на ней, назовем потоком электроёмкостной самоиндукции. Он изменяется путем изменения разности потенциалов при постоянной емкости, или изменения емкости при постоянной разности потенциалов, или того и другого.

Если величина емкости или разности потенциалов на ёмкости зависят от времени, то величина тока определяется соотношением:

$$
I=\frac{d Q_{C, U}}{d t}=C \frac{\partial U}{\partial t}+U \frac{\partial C}{\partial t} .
$$

Это выражение определяет закон электрической самоиндукции. Таким образом, ток в цепи, содержащей конденсатор, можно получить двумя способами, изменяя напряжение на конденсаторе при постоянной его ёмкости или изменяя саму ёмкость при неизменном напряжении на конденсаторе, или производить изменение обоих параметров одновременно.

При постоянной емкости $C_{1}$ известно выражение для тока через емкость:

$$
I=C_{1} \frac{\partial U}{\partial t} .
$$

Если изменяется емкость, и напряжение $U_{1}$ на ней неизменно, то имеем:

$$
I=U_{1} \frac{\partial C}{\partial t} .
$$

Это параметрическая самоиндукция - ток связан с изменением ёмкости.

Рассмотрим следствия из (11.2). Если к емкости подключить генератор постоянного тока $I_{0}$, то напряжение на ней будет изменяться по закону: 


$$
U=I_{0} t / C_{1},
$$

то есть емкость, подключенная к источнику постоянного тока, представляет для него линейно зависящее от времени активное сопротивление [34,35]

$$
R=t / C_{1} .
$$

Физический смысл этого - расход энергии на зарядку емкости.

Мощность, отдаваемая источником тока, в данном случае равна

$$
P(t)=I_{0}{ }^{2} t / C_{1} \text {. }
$$

Накопленную емкостью за время $t$ энергию получим, интегрируя (11.6):

$$
W_{C}=I_{0}^{2} t^{2} /\left(2 C_{1}\right) \text {. }
$$

Подставляя сюда значение тока из (11.4), получаем зависимость величины накопленной в емкости энергии от текущего значения напряжения на ней:

$$
W_{C}=C_{1} U^{2} / 2 \text {. }
$$

Используя для данного случая понятие потока электрической индукции

$$
\Phi_{U}=C_{1} U=Q(U)
$$

и используя соотношение (11.2), получаем:

$$
I_{0}=\frac{d \Phi_{U}}{d t}=\frac{\partial Q(U)}{\partial t}
$$

т.е., если к постоянной емкости подключить источник постоянного тока, то ток будет равен производной потока ёмкостной индукции по времени.

Теперь пусть напряжение $U_{1}$ постоянно на переменной емкости, тогда

$$
I=U_{1} \frac{\partial C}{\partial t}
$$

Видно, что величина

$$
R_{C}=\left(\frac{\partial C}{\partial t}\right)^{-1}
$$

играет роль активного сопротивления [34, 35], что физически понятно, т.к. при увеличении емкости увеличивается накопленная в ней энергия, ёмкость отбирает у источника напряжения энергию, представляя для него активную нагрузку. Мощность, расходуемая при этом источником, равна

$$
P(t)=\frac{\partial C}{\partial t} U_{1}^{2}
$$

Из (11.11) видно, что в зависимости от знака производной расходуемая мощность имеет разные знаки. Когда производная положительная, расходуемая мощность идёт на совершение внешней работы. Если производная отрицательная, то работу совершает внешний источник, заряжая ёмкость.

Опять, вводя понятие потока электрической индукции

$$
\Phi_{C}=C U_{1}=Q(C),
$$

получаем

$$
I=\frac{\partial \Phi_{C}}{\partial t}
$$

Соотношения (11.8) и (11.12) указывают на то, что независимо от способа изменения потока, его производная по времени всегда равна току. 
Рассмотрим еще один процесс, который ранее к законам индукции не относили, однако, он подпадает под наше расширенное определение этого понятия. Из (11.7) видно, что если поток, т.е. заряд, оставить неизменным (назовем это режимом замороженного электрического потока), то напряжение на емкости можно изменять путем ее изменения. Тогда имеем:

$$
C U=C_{0} U_{0}=\text { const }
$$

где $C$ и $U$ - текущие значения, а $C_{0}$ и $U_{0}$ - начальные значения этих параметров, имеющие место при отключении от емкости источника питания.

Напряжение на емкости и накопленная в ней энергия равны

$$
U=C_{0} U_{0} / C, W_{C}=\left(C_{0} U_{0}\right)^{2} /(2 C) .
$$

Данная самоиндукция связана только с изменением самой емкости, поэтому подпадает под определение параметрической самоиндукции.

Итак, (11.8), (11.12) и (11.13), определяют процессы электрической самоиндукции. Назовем их правилами электрического потока. Соотношение (11.8) определяет электрическую самоиндукцию без изменения емкости, и она может быть названа просто электрической самоиндукцией. Соотношения (11.3) и (11.9)-(11.11) предполагают изменение емкости, поэтому такие процессы назовем электрической параметрической самоиндукцией.

\section{§ 12. Индуктивнотоковая самоиндукция}

Перейдем теперь к рассмотрению процессов, происходящих в индуктивности. Введем понятие потока токовой самоиндукции

$$
\Phi_{L, I}=L I .
$$

Если индуктивность закорочена и выполнена из материала, не имеющего активного сопротивления, например, из сверхпроводника, то

$$
\Phi_{L, I}=L_{1} I_{1}=\text { const }
$$

где $L_{1}$ и $I_{1}$ - какие-то начальные значения этих параметров, которые имеются в момент короткого замыкания индуктивности при наличии в ней тока. Этот режим назовем законом замороженного потока для короткозамкнутых сверхпроводящих контуров $[34,35]$. При этом имеем:

$$
I=I_{1} L_{1} / L
$$

где $I$ и $L$ - текущие значения соответствующих параметров.

В данном режиме поток индуктивнотоковой индукции неизменен, однако в связи с тем, что ток в индуктивности может изменяться при ее изменении, такой процесс подпадает под определение параметрической самоиндукции. Энергия, накопленная в индуктивности, при этом равна

$$
W_{L}=\frac{1}{2} \frac{\left(L_{1} I_{1}\right)^{2}}{L}=\frac{1}{2} \frac{(\text { const })^{2}}{L} .
$$

Напряжение на индуктивности равно производной потока токовой индукции по времени:

$$
U=\frac{d \Phi_{L, I}}{d t}=L \frac{\partial I}{\partial t}+I \frac{\partial L}{\partial t}
$$


Рассмотрим случай, когда индуктивность $L_{1}$ постоянна, тогда

$$
U=L_{1} \frac{\partial I}{\partial t} \text {. }
$$

Обозначая $\Phi_{I}=L_{1} I$, получим $U=\frac{d \Phi_{I}}{d t}$. Проинтегрируем (12.2) по времени:

$$
I=U t / L_{1} .
$$

Таким образом, индуктивность, подключенная к источнику постоянного напряжения, представляет для него активное сопротивление [34, 35]

$$
R=L_{1} / t
$$

которое уменьшается обратно пропорционально времени.

Расходуемая источником мощность линейно зависит от времени:

$$
P(t)=U^{2} t / L_{1} \text {. }
$$

Интеграл от (12.5) по времени есть накопленная в индуктивности энергия:

$$
W_{L}=U^{2} t^{2} /\left(2 L_{1}\right)
$$

Подставив в (12.6) значение напряжения из соотношения (12.3), получаем:

$$
W_{L}=L_{1} I^{2} / 2 \text {. }
$$

Эта энергия может быть возвращена из индуктивности во внешнюю цепь, если индуктивность отключить от источника питания и подключить к ней активное сопротивление.

Теперь рассмотрим случай, когда ток $I_{1}$, протекающий через индуктивность, постоянен, а сама индуктивность может изменяться. Тогда имеем:

$$
U=I_{1} \frac{\partial L}{\partial t}
$$

Следовательно, величина

$$
R(t)=\partial L / \partial t
$$

играет роль активного сопротивления [34,35]. Как в случае электрического потока, активное сопротивление может быть (в зависимости от знака производной), и положительным, и отрицательным, то есть индуктивность может, как получать энергию извне, так и отдавать её во внешние цепи.

Вводя обозначение $\Phi_{L}=L I_{1}$ и, учитывая (12.7), получаем:

$$
U=d \Phi_{L} / d t .
$$

Соотношения (12.1), (12.6) и (12.9) назовем правилами токовой самоиндукции, или правилами потока токовой самоиндукции. Из (12.6) и (12.9) видно, что, как в случае с электрическим потоком, способ изменения токового потока не влияет на конечный результат, и его производная по времени всегда равна приложенной разности потенциалов. Соотношение (12.6) определяет токовую самоиндукцию, при которой отсутствуют изменения индуктивности, поэтому назовем ее просто токовой самоиндукцией. Соотношения (12.7), (12.8) предполагают наличие изменений индуктивности, такие процессы назовем токовой параметрической самоиндукцией.

Закон замороженного тока (12.1) приводит к неизвестному ранее явлению магнитной потенциальной ямы на сверхпроводящих кольцах. 
Пусть в двух соосно расположенных сверхпроводящих кольцах заморожены токи, причем ток в нижнем кольце значительно больше, чем в верхнем. В соответствии с законом Био-Савара магнитная индукция нижнего кольца на оси в плоскости верхнего кольца имеет вид:

$$
B=\frac{\mu_{0} I_{1} R_{1}^{2}}{2\left(R_{1}^{2}+z_{0}^{2}\right)^{3 / 2}},
$$

где $\mu_{0}$ - магнитная постоянная, $z_{0}$ - расстояние между кольцами, $R_{1}$ - диаметр нижнего кольца, $I_{1}$ - ток, замороженный в нижнем кольце.

Если радиус верхнего кольца составляет $R_{2}$, и его диаметр значительно меньший, чем радиус нижнего кольца, то магнитный поток, создаваемый нижним кольцом и пронизывающий верхнее кольцо, составит

$$
\Phi_{2} \cong \frac{\mu_{0} I_{1} R_{1}^{2} R_{2}^{2}}{2\left(R_{1}^{2}+z_{0}^{2}\right)^{3 / 2}} .
$$

Будем считать, что расстояние между кольцами значительно больше, чем диаметр нижнего кольца, тогда

$$
\Phi_{2} \cong \mu_{0} I_{1} R_{1}^{2} R_{2}^{2} /\left(2 z_{0}^{3}\right) .
$$

Если в верхнем кольце заморожен ток $I_{2}$, то с ним связан поток

$$
\Phi_{2}=L_{2} I_{2}
$$

где $L_{2}$ - индуктивность верхнего кольца.

Допустим, что в начальном положении колец направление токов в них совпадает, и кольца притягиваются. При опускании нижнего кольца токи индукции будут компенсировать токи в верхнем кольце, и ток в нём достигнет нулевого значения, когда будет выполняться равенство

$$
\Phi_{2} \cong \frac{\mu_{0} I_{1} R_{1}^{2} R_{2}^{2}}{2 z_{0}^{3}} .
$$

При этом расстояние между кольцами составит

$$
z_{0}=\left(\frac{\mu_{0} I_{1} R_{1}^{2} R_{2}^{2}}{2 \Phi_{2}}\right)^{1 / 3}=\left(\frac{\mu_{0} I_{1} R_{1}^{2} R_{2}^{2}}{2 L_{2} I_{2}}\right)^{1 / 3} .
$$

При получении этого соотношения не учтена взаимная индуктивность колец, т.к. расстояние между кольцами велико, а также считалось, что ток $I_{1}$ практически не меняется при приближении верхнего кольца к нижнему.

$\mathrm{B}$ точке $z_{0}$ токи индукции полностью компенсируют ток, замороженный в верхнем кольце, следовательно, это кольцо уже не будет взаимодействовать с нижним кольцом. При дальнейшем сближении колец ток индукции в верхнем кольце поменяет своё первоначальное направление и будет противоположен по направлению току в нижнем кольце, а, следовательно, начнёт от него отталкиваться. Если же кольца будут удаляться друг от друга, то в верхнем кольце возникнут ток, совпадающие по направлению с токами в нижнем кольце, и кольца будут притягиваться. Таким образом, по- 
ложение верхнего кольца в точке $z_{0}$ есть потенциальная яма. Такие свойства сверхпроводящих подвесов в литературных источниках не описаны.

Этот анализ принципиальной возможности получения потенциальной ямы на сверхпроводящих кольцах с замороженными токами не учитывает взаимной индуктивности $M(z)$ колец, которая для двух круговых контуров радиуса $R_{1}$ и $R_{2}$ с общей осью и расстоянием $z$ между ними выражается через полные эллиптические интегралы 1-го $K(k)$ и 2-го $E(k)$ рода:

$$
\begin{gathered}
M(z)=\mu_{0} f(k(z)) \sqrt{R_{1} R_{2}}, \\
f(k)=\left(\frac{2}{k}-k\right) K(k)-\frac{2}{k} E(k)=\frac{2}{k}\left[\left(1-\frac{k^{2}}{2}\right) K(k)-E(k)\right], k^{2}=\frac{4 R_{1} R_{2}}{z^{2}+\left(R_{1}+R_{2}\right)^{2}}, \\
K(k)=\int_{0}^{\frac{\pi}{2}} \frac{d \theta}{\sqrt{1-k^{2} \sin ^{2} \theta}}, E(k)=\int_{0}^{\frac{\pi}{2}} \sqrt{1-k^{2} \sin ^{2} \theta} d \theta .
\end{gathered}
$$

В дальнейшем для расчётов будут использованы также собственные индуктивности колец с радиусами сечения проводов $r_{1}$ и $r_{2}$ :

$$
L_{10}=\mu_{0} R_{1}\left(\ln \frac{8 R_{1}}{r_{1}}-1.75\right), L_{20}=\mu_{0} R_{2}\left(\ln \frac{8 R_{2}}{r_{2}}-1.75\right) .
$$

Заморозим токи $I_{10}, I_{20}$ в двух уединённых сверхпроводящих кольцах с собственными индуктивностями $L_{10}, L_{20}$. Расположим эти кольца соосно на большом расстоянии так, чтобы направления токов в них опять совпадали и начнём их сближать. Тогда имеем соотношения, выражающие закон сохранения замороженных потоков в сверхпроводящих кольцах:

$$
L_{10} I_{1}(z)+M(z) I_{2}(z)=L_{10} I_{10} ; \quad L_{20} I_{2}(z)+M(z) I_{1}(z)=L_{20} I_{20} .
$$

Из них получим соотношения для токов:

$$
I_{1}(z)=\frac{I_{10} L_{10} L_{20}-I_{20} L_{20} M(z)}{L_{10} L_{20}-M^{2}(z)} ; \quad I_{2}(z)=\frac{I_{20} L_{10} L_{20}-I_{10} L_{10} M(z)}{L_{10} L_{20}-M^{2}(z)} .
$$

Из этих соотношений получим значение взаимной индуктивности, при которой ток в нижнем и верхнем кольцах равен нулю:

$$
M_{1}\left(z_{1}\right)=L_{1} \frac{I_{10}}{I_{20}} ; \quad M_{2}\left(z_{2}\right)=L_{2} \frac{I_{20}}{I_{10}} .
$$

Таким образом, задавая различные начальные значения индуктивностей и замороженные в них токи, можно задавать нулевые значения токов в том или другом кольце при различных расстояниях между ними.

На рис. 6 и рис. 7 изображены рассчитанные по формулам (12.11) графики $I_{1}(z)$ и $I_{2}(z)$ при различных начальных значениях токов в кольцах радиусов $R_{1}=10 \mathrm{~cm}, R_{2}=8 \mathrm{~cm}$ (радиусы сечения проводов $a_{1}=a_{2}=0,1 \mathrm{~cm}$ ). Видно, что на определённом расстоянии от нижнего кольца ток в верхнем меняет знак. На большем расстоянии направления токов в кольцах совпадают, и они притягиваются. На меньшем - направления противоположны, и они отталкиваются. Следовательно, точка, в которой ток в верхнем кольце меняет знак, есть координата потенциальной ямы. 


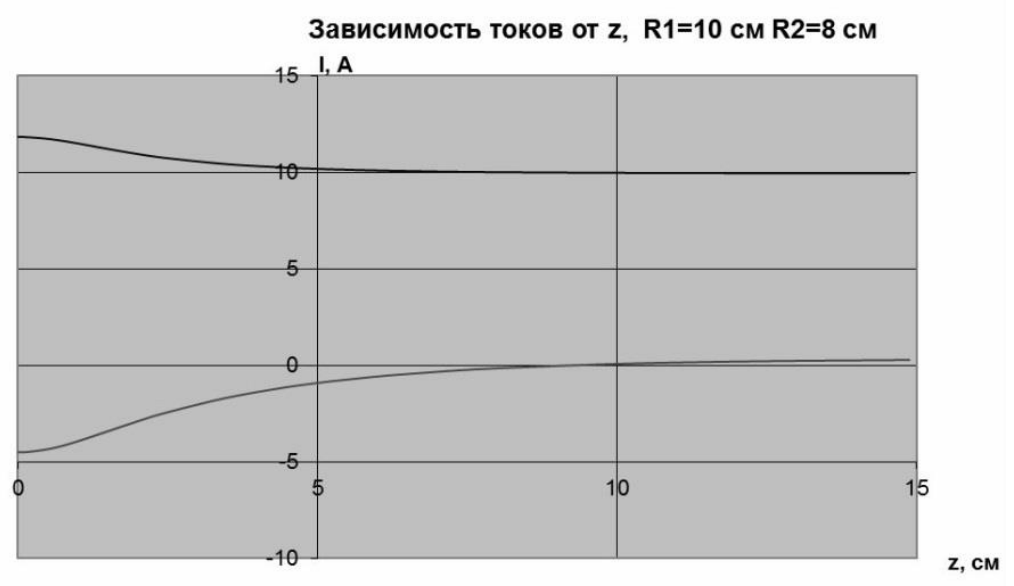

Рис. 6. Графики токов в нижнем кольце (верхняя кривая) и в верхнем кольце (нижняя кривая) при начальных значениях силы тока в нижнем кольце 10 А и значении силы тока в верхнем кольце - 2 А.

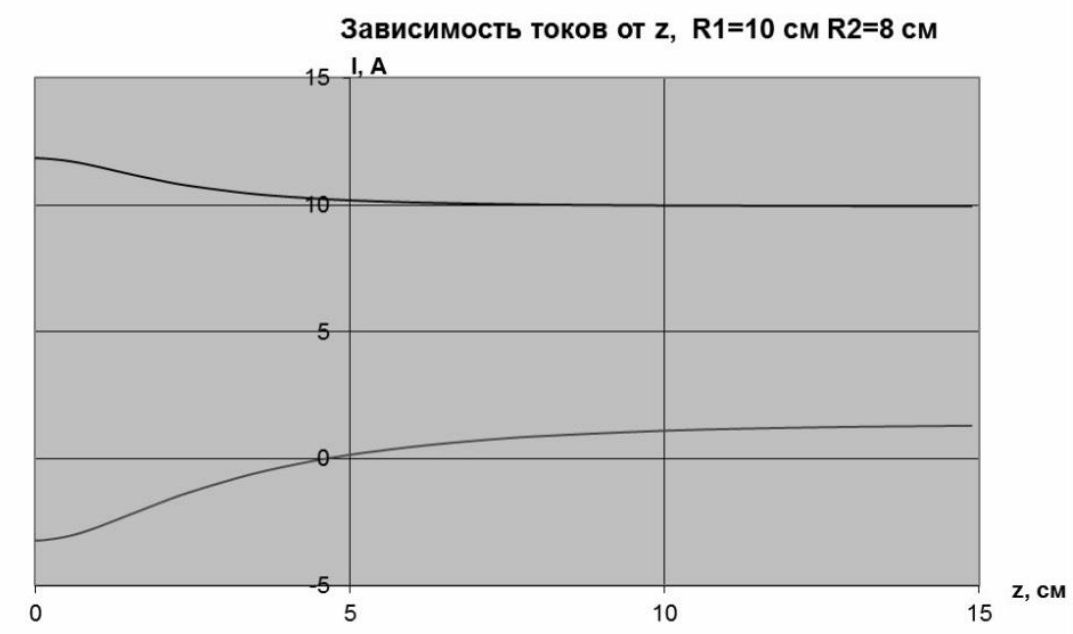

Рис. 7. Графики токов в нижнем кольце (верхняя кривая) и в верхнем кольце (нижняя кривая) при начальных значениях силы тока в нижнем кольце 10 А и значении силы тока в верхнем кольце $-5 \mathrm{~A}$.

Идея создания потенциальной ямы на сверхпроводящих кольцах принадлежит авторам монографии, а (12.11) получены Иваном Фёдоровичем Гориным - участником научного форума Движения за возрождение отечественной науки (http://www.forum.za-nauku.ru/index.php/board,6.0.html)

Участник этого же форума Николай Александрович Дробышев представил решение конкретной задачи, которое приводим ниже без купюр.

Задача: Оси двух колец из сверхпроводящих проводов круглого сечения совпадают и направлены вдоль $O z$, при этом начало отсчета $O$ совмещено с центром первого кольца. Найти токи в кольцах и силу взаимодействия колец в зависимости от координаты центра второго кольца $z$, если при $z=z_{0}$ ток во втором кольце отсутствует, а в первом равен $I$. Радиусы колец $r_{1}$ и $r_{2}$ намного больше радиусов $a_{1}$ и $a_{2}$ сечения проводов.

Ответ: Ток в первом кольце 


$$
I_{1}(z)=I \frac{b_{1} b_{2}-4 f\left(\kappa_{0}\right) f(\kappa)}{b_{1} b_{2}-4[f(\kappa)]^{2}}
$$

а во втором -

$$
I_{2}(z)=2 I b_{1} \sqrt{\frac{r_{1}}{r_{2}}} \frac{f\left(\kappa_{0}\right)-f(\kappa)}{b_{1} b_{2}-4[f(\kappa)]^{2}} ;
$$

сила взаимодействия колец

$$
F(z)=\mu_{0} I^{2} g(\kappa) \frac{\left[b_{1} b_{2}-4 f\left(\kappa_{0}\right) f(\kappa)\right]\left[f\left(\kappa_{0}\right)-f(\kappa)\right]}{\left[b_{1} b_{2}-4[f(\kappa)]^{2}\right]^{2}} \frac{b_{1}|z|}{r_{2}} .
$$

Здесь $\mu_{0}$ - магнитная постоянная, $b_{1}=\ln \left(8 r_{1} / a_{1}\right)-2$ и $b_{2}=\ln \left(8 r_{2} / a_{2}\right)-2$, а

$$
\kappa=\kappa(z)=2 \sqrt{\frac{r_{1} r_{2}}{\left(r_{1}+r_{2}\right)^{2}+z^{2}}}, \quad \kappa_{0}=\kappa\left(z_{0}\right)=2 \sqrt{\frac{r_{1} r_{2}}{\left(r_{1}+r_{2}\right)^{2}+z_{0}^{2}}} .
$$

В выражениях (1)-(3) фигурируют функции

$$
f(\kappa)=\frac{1}{\kappa}\left[\left(1-\kappa^{2} / 2\right) K(\kappa)-E(\kappa)\right], \quad g(\kappa)=\kappa\left[K(\kappa)-\frac{1-\kappa^{2} / 2}{1-\kappa^{2}} E(\kappa)\right],
$$

где $K(\kappa)$ и $E(\kappa)$ - полные эллиптические интегралы 1-го и 2-го рода:

$$
K(\kappa)=\int_{0}^{\pi / 2} \frac{d \theta}{\sqrt{1-\kappa^{2} \sin ^{2} \theta}}, \quad E(\kappa)=\int_{0}^{\pi / 2} \sqrt{1-\kappa^{2} \sin ^{2} \theta} d \theta .
$$

В качестве примера возьмем следующие радиусы колец: $r_{1}=10$ см и $r_{2}=8 \mathrm{~cm}$, а радиусы сечения проводов $-a_{1}=a_{2}=0,1 \mathrm{~cm}$. Пусть при расстоянии $z_{0}=5$ см между центрами колец сила тока в первом кольце составляет $I=10 \mathrm{~A}$, а во втором ток отсутствует. Зависимости $I_{1}(z), I_{2}(z)$ и $F(z)$, рассчитанные по формулам (1)-(3), изображены на графиках. Ток в первом кольце $I_{1}$ равен $I=10$ А при $z= \pm z_{0}$ и $z \rightarrow \pm \infty$. Ток во втором кольце $I_{2}$ обращается в нуль при $z= \pm z_{0}$.

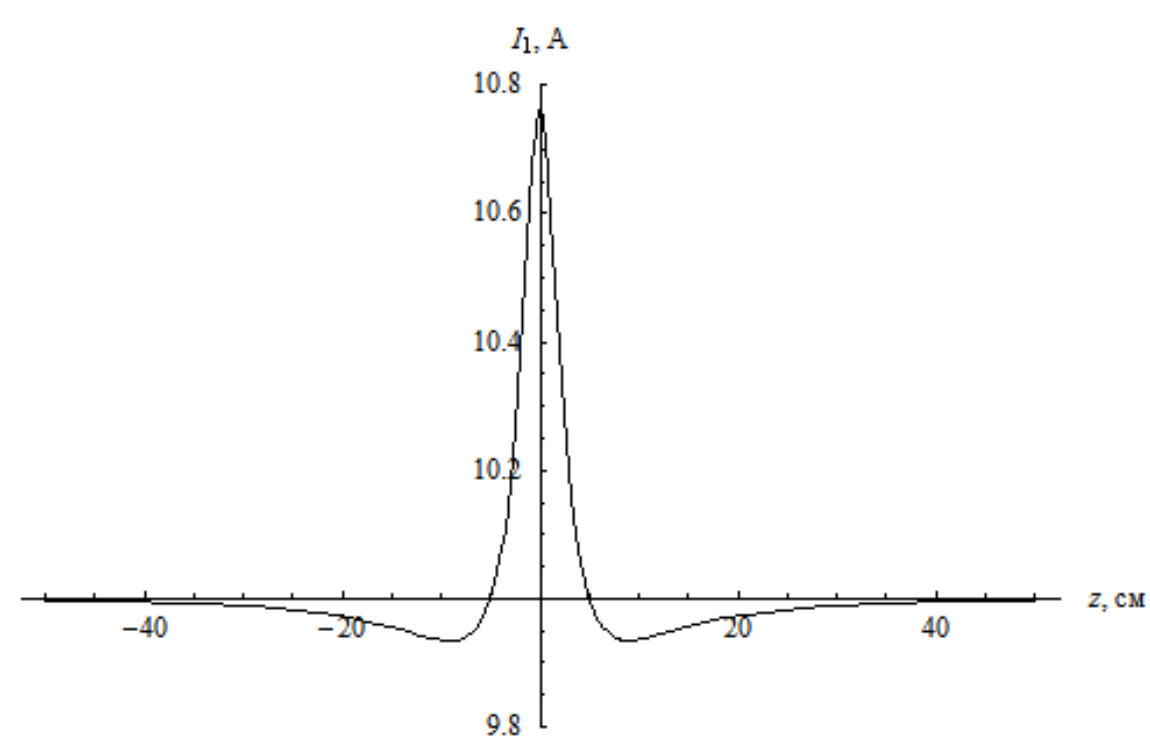



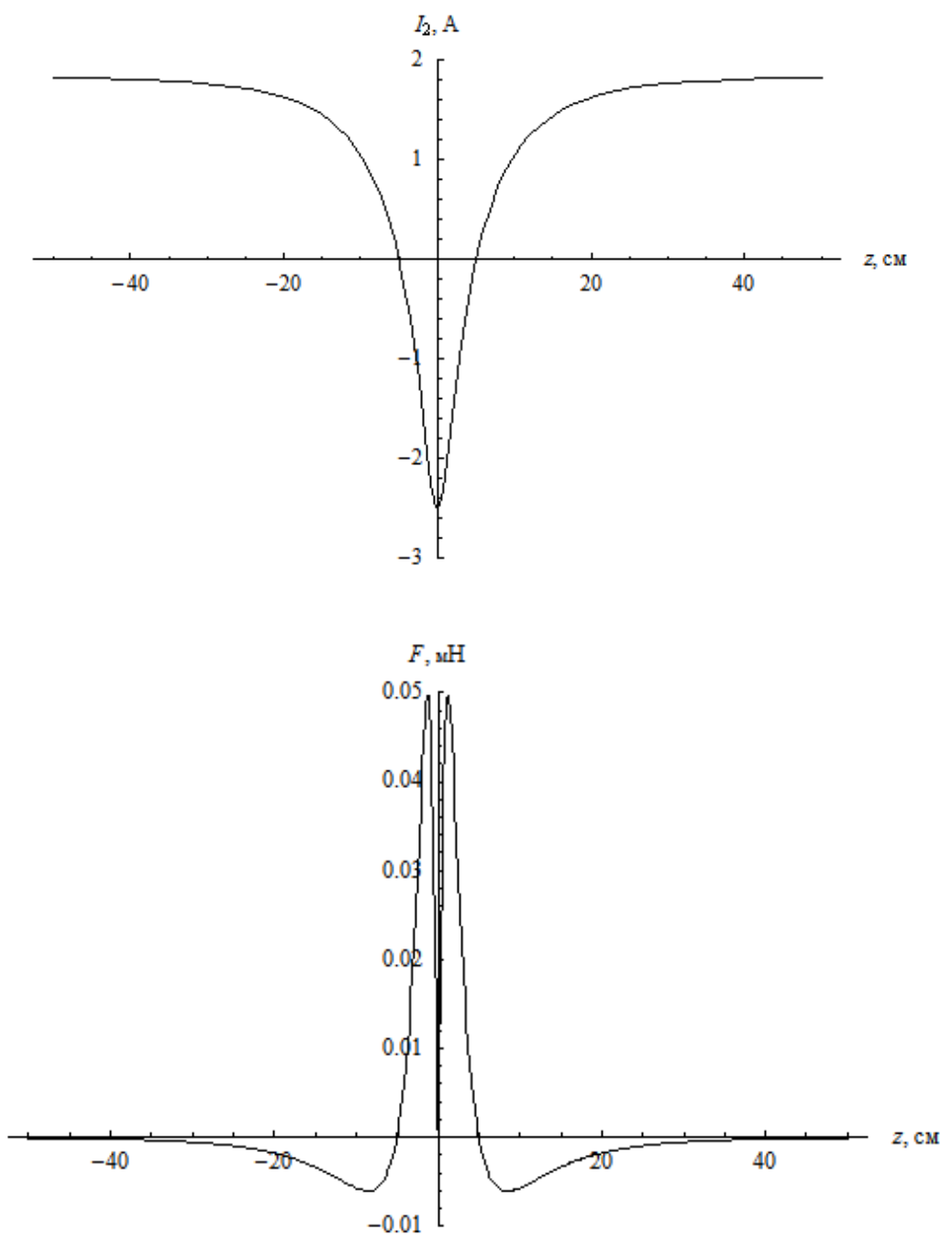

Положительные значения $I_{2}$ соответствуют одинаковым направлениям токов в кольцах, а отрицательные - противоположным. Наконец, положительные значения силы $F$ соответствуют отталкиванию колец, а отрицательные - притяжению. Очевидно, что при $z= \pm z_{0}$ она обращается в ноль, при этом кольца находятся в положении устойчивого равновесия. Сила $F$ отсутствует также при $z=0$ (когда плоскости колец совпадают), но в этом случае равновесие - неустойчивое.

Данный факт свидетельствует о большой полезности сотрудничества учёных на указанном научном форуме, когда одни предлагают идею, а другие находят её математическое воплощение.

\section{§ 13. Новый способ получения волнового уравнения, потенциальные и кинетические потоки зарядов}

В двух предыдущих параграфах рассматривались процессы в цепях с сосредоточенными параметрами (распределение разностей потенциалов и токов в элементах принималось однородным). Процессы же в цепях с пространственно-неоднородными разностями потенциалов и токами (например, длинные линии) описываются волновыми уравнениями, которые мо- 
гут быть получены из уравнений Максвелла или при помощи телеграфных уравнений, но физика самого явления остаётся не ясной [36].

Обозначим погонную (приходящуюся на единицу длины) емкость и индуктивность такой линии через $C_{0}$ и $L_{0}$. При подключении к ней источника постоянного напряжения $U_{1}$ координата $z$ фронта распространяется в линии с какой-то скоростью $v$ по закону $z=v t$. При этом суммарная величина заряженной ёмкости и величина суммарной индуктивности, по которой протекает ток, отсчитываемые от начала линии до места нахождения фронта напряжения, изменяются по закону [36]:

$$
C(t)=z C_{0}=v t C_{0}, L(t)=z L_{0}=v t L_{0} .
$$

Источник напряжения $U_{1}$ заряжает увеличивающуюся емкость линии, для чего от источника к заряжаемой линии в соответствии с (11.9) течёт ток:

$$
I_{1}=U_{1} \frac{\partial C(t)}{\partial t}=v U_{1} C_{0} .
$$

Он течёт через проводники линии, обладающие индуктивностью. Но, поскольку индуктивность линии в связи с движением фронта напряжения тоже увеличивается, то в силу (12.20) на ней будет падение напряжения

$$
U=I_{1} \frac{\partial L(t)}{\partial t}=v I_{1} L_{0}=v^{2} U_{1} C_{0} L_{0} .
$$

Абсолютная величина падения напряжения на проводниках линии равна напряжению на её входе: $U=U_{1}$. Поэтому скорость распространения электротоковых колебаний в линии (скорость фронта напряжения) равна

$$
v=1 / \sqrt{L_{0} C_{0}} .
$$

Следовательно, при подключении источника напряжения к бесконечно длинной линии в ней имеет место саморасширение электрических полей и токов, заполняющих линию энергией, и скорость фронта постоянного напряжения и тока равна скорости распространения электромагнитных колебаний в такой линии. Такую волну назовём электротоковой. Интересно, что результат не зависит от вида функции $U$, т.е. при подключении к линии источника напряжения, меняющегося по любому закону или постоянного, локальное значение напряжения на входе линии распространяется вдоль неё со скоростью (13.2). Этот результат мог быть до сих пор получен только решением волнового уравнения. В данном же случае раскрыта физическая причина такого распространения, дана физическая картина процесса - заполнение линии электрической и токовой энергией, происходящее так, что фронт волны, распространяясь со скоростью $v$, оставляет за собой линию, заряженную до разности потенциалов $U_{1}$, что соответствует заполнению линии электростатической энергией электрического поля. На участке же линии от источника напряжения до фронта волны течет ток $I_{1}$, что соответствует заполнению этого участка линии энергией, связанной с движением зарядов по проводникам линии, обладающих индуктивностью.

Величину тока в линии можно получить подстановкой (13.2) в (13.1):

$$
I_{1}=U_{1} \sqrt{C_{0} / L_{0}}=U_{1} / Z
$$


где $Z=\sqrt{L_{0} / C_{0}}$ - волновое сопротивление линии.

В данном случае

$$
U_{1}=I \frac{\partial L}{\partial t}=\frac{d \Phi_{L}}{d t}, I_{1}=U_{1} \frac{\partial C}{\partial t}=\frac{d \Phi_{C}}{d t} .
$$

Видно, что правила потока и для электрической, и для токовой самоиндукции соблюдаются и в этом случае.

Таким образом, процессы распространения разности потенциалов вдоль проводников длинной линии и постоянного тока в ней связаны, взаимно дополняют друг друга и не могут существовать друг без друга. Назовём это электротоковой самопроизвольной параметрической самоиндукцией. Такое название связано с тем, что расширение потоков самопроизвольно и характеризует скорость процесса заполнения линии энергией. Прояснилась связь между энергетическими процессами и скоростью распространения фронтов волны в длинных линиях. Поскольку при излучении электромагнитных волн свободное пространство тоже является передающей линией, подобные законы должны характеризовать распространение и в нём.

Если одним из проводников длинной линии взять спираль (длинный соленоид), скорость распространения фронта напряжения в линии уменьшится, поскольку её погонная индуктивность увеличится. Такому распространению будет сопутствовать распространение не только внешних по отношению к соленоиду полей и токов, но и распространение магнитного потока внутри соленоида, и скорость распространения такого потока будет равна скорости распространения электромагнитной волны в самой линии.

Зная ток и напряжение в линии, вычислим удельные энергии, заключенную в погонной ёмкости и индуктивности линии:

$$
\begin{gathered}
W_{C}=C_{0} U_{1}^{2} / 2, \\
W_{L}=L_{0} I_{1}^{2} / 2 .
\end{gathered}
$$

Нетрудно видеть, что $W_{C}=W_{L}$.

Длительность фронта электротоковой волны определяется свойствами источника напряжения, т.к. локальная производная $\partial U / \partial t$ на входе линии зависит от переходных процессов в источнике и в устройстве, при помощи которого источник подключается к линии. Если установление напряжения на входе линии длится время $\Delta t$, то в линии оно займет участок длиной $v \Delta t$. Приложенное к линии напряжение $U(t)$ будет наблюдаться в любой её точке на расстоянии $z$ от ее начала с запаздыванием $t=z / v$. Функция

$$
U(t, z)=U(t-z / v)
$$

может быть названа функцией распространения, т.к. она связывает локальные временные и пространственные значения. Длинная линия есть устройство преобразования локальных производных напряжения по времени на входе линии в пространственные производные в самой линии:

$$
\frac{\partial U(z)}{\partial z}=\frac{1}{v} \frac{\partial U(t)}{\partial t}
$$


Отметим, что распространение обязано естественному саморасширению электрического поля и тока в линии и подчиняется правилам параметрической самоиндукции. Для решения волновых уравнений длинных линий

$$
\frac{\partial^{2} U}{\partial z^{2}}=\frac{1}{v^{2}} \frac{\partial^{2} U}{\partial t^{2}} ; \quad \frac{\partial^{2} I}{\partial z^{2}}=\frac{1}{v^{2}} \frac{\partial^{2} I}{\partial t^{2}},
$$

полученных из телеграфных уравнений

$$
\frac{\partial U}{\partial z}=-L \frac{\partial I}{\partial t} ; \quad \frac{\partial I}{\partial z}=-C \frac{\partial U}{\partial t},
$$

требуется знание вторых производных напряжений и токов.

Уравнения (13.6) не отвечают на вопрос, как быть при подаче на вход линии напряжения с нулевой второй производной (напряжение источника меняется по линейному закону)? Используемый метод даёт такой ответ.

При рассмотрении процессов в длинной линии фигурировали понятия погонной емкости и индуктивности, а также тока и напряжения в линии, но отсутствовали понятия электрического и магнитного поля. Однако уравнения Максвелла используют понятия электрической и магнитной проницаемости среды, а не емкости и индуктивности. Покажем, как перейти к понятийному аппарату уравнений Максвелла. Для этого возьмем простейшую конструкцию линии, расположенную в вакууме, как показано на pис. 2. Будем считать, что $b>>a$ и краевые эффекты можно не учитывать. Тогда между погонными параметрами линии и магнитной и диэлектрической проницаемостями будет существовать следующая связь:

$$
\begin{aligned}
& L_{0}=\mu_{0} a / b, \\
& C_{0}=\varepsilon_{0} b / a,
\end{aligned}
$$

где $\mu_{0}$ и $\varepsilon_{0}$ - магнитная и диэлектрическая проницаемость вакуума.

Фазовая скорость в такой линии будет определяться соотношением:

$$
v=1 / \sqrt{L_{0} C_{0}}=1 / \sqrt{\mu_{0} \varepsilon_{0}}=c,
$$

где $c$ - скорость света в вакууме.

Волновое сопротивление рассмотренной линии равно

$$
Z=\frac{a}{b} \sqrt{\mu_{0} / \varepsilon_{0}}=\frac{a}{b} Z_{0},
$$

где $Z_{0}=\sqrt{\mu_{0} / \varepsilon_{0}}$ - волновое сопротивление свободного пространства.

При $a=b$ получаем $L_{0}=\mu_{0}$, то есть $\mu_{0}$ играет роль продольной удельной индуктивности вакуума. Тогда $C_{0}=\varepsilon_{0}$, то есть $\varepsilon_{0}$ играет роль поперечной удельной ёмкости вакуума. Такая интерпретация проясняет физический смысл $\mu_{0}$, и $\varepsilon_{0}$. Так же, как в длинной линии, они обеспечивают процесс распространения электромагнитной волны в свободном пространстве.

Рассмотрение электромагнитной волны в длинной линии можно рассматривать как заполнение пространства, находящегося между её проводниками, особым видом материи, которую представляют электрические и магнитные поля. Математически можно считать, что эти поля сами обладают удельной энергией, и при их помощи можно передавать энергию по линиям передач. Если же рассматривать процессы, протекающие при из- 
лучении электромагнитных волн с помощью антенны, то его можно рассматривать также как заполнение свободного пространства этим видом материи. Однако геометрия полей и токов в этом случае сложнее, поскольку всегда присутствуют как поперечные, так и продольные составляющие полей. Такой подход исключает необходимость применения для описания распространения электромагнитных волн такой субстанции, как эфир.

Если к рассмотренной линии бесконечной длины или линии, нагруженной волновым сопротивлением, подключить источник постоянного напряжения $U$, то напряженность поля в линии составит

$$
E_{y}=U / a,
$$

а ток, текущий в линию от источника питания, равен

$$
I=U / Z=a E_{y} / Z \text {. }
$$

Магнитное поле в линии равно удельному току, протекающему в линии

$$
H_{x}=I / b=a E_{y} /(b Z) .
$$

Подставляя сюда значение $Z$, получаем

$$
H_{x}=E_{y} / Z_{0} \text {. }
$$

Так же связаны электрическое и магнитное поля в поперечных электромагнитных волнах, распространяющихся в свободном пространстве.

Сравнивая выражения для энергий, нетрудно видеть, что удельные энергии могут быть выражены через электрические и магнитные поля

$$
\mu_{0} H_{x}^{2} / 2=\varepsilon_{0} E_{y}^{2} / 2 \text {. }
$$

Это означает, что удельная энергия, накопленная в магнитном и электрическом поле в такой линии, одинакова. Если эти энергии умножить на объемы, занимаемые полями, то получим совпадение с (13.3-13.4).

Таким образом, в рассмотренной линии распространяются такие же поперечные плоские волны, как в свободном пространстве. Этот вывод получен не решением уравнений Максвелла, а рассмотрением динамических процессов, относящихся к параметрической самоиндукции. Особенность такой линии - в ней, в отличие от свободного пространства, могут распространяться постоянные магнитные и электрические поля, а этот случай не может быть рассмотрен путем решения уравнений Максвелла.

При подключении к длинной линии источника постоянного напряжения она заполняется двумя видами энергии: электрической и магнитной. Их удельные плотности равны, а поскольку они заполняют одинаковые объемы, то и накопленная в этих полях общая энергия одинакова. Особенностью данной линии является то, что при протекании в линии постоянного тока распределение электрического и магнитного полей в ней является однородным. Из (13.11), где правая и левая части представляют приложенные к плоскостям линии удельные силы, следует, что действующая на проводники такой линии сила равна нулю. Электрическая и магнитная силы компенсируют друг друга, имея разные знаки. Этот вывод касается и передающих линий любой другой конфигурации.

Если к линии приложить напряжение, меняющееся со временем по любому закону $U(t)=a E_{y}(t)$, то, по аналогии с (13.5), можно записать: 


$$
E_{y}(z)=E_{y}(t-z / c)
$$

Аналогичное соотношение будет и для магнитных полей.

Мощность $P$, передаваемая через поперечное сечение линии в направлении $z$, равна $I(t) U(t)$. Если ток и напряжение выразить через напряженности магнитного и электрического полей, то получим $P=a b E_{y} H_{x}$. Произведение $E_{y} H_{x}$ есть абсолютная величина вектора Пойнтинга, представляющего удельную мощность, передаваемую через поперечное сечение линии единичной площади. Всё это можно записать и в векторной форме.

Таким образом, при рассмотрении процессов в длинной линии двумя методами все выводы совпадают. Поэтому и в дальнейшем, не рискуя принципиально ошибиться, можно для описания процессов в длинных линиях с успехом пользоваться такими параметрами, как распределенная индуктивность и ёмкость. При этом следует понимать, что $C_{0}$ и $L_{0}$ - это некоторые интегральные характеристики, не учитывающие структуру полей. Следует отметить, что, с практической точки зрения, применение параметров $C_{0}$ и $L_{0}$ имеет важное значение, т.к. могут быть приближенно решены задачи, которые при помощи уравнений Максвелла решить нельзя. Это, например, случай, когда проводниками передающей линии являются спирали.

Важность полученных результатов заключается в том, что можно, не прибегая к уравнениям Максвелла, решать задачи распространения. Также показано, что в длинных линиях и в свободном пространстве электромагнитные процессы распространяются с конечной скоростью.

Указанные закономерности распространяются на все виды передающих линий. Для различного типа линий погонные параметры зависят от их размеров. Для примера рассмотрим коаксиальную линию, у которой погонная ёмкость и индуктивность даётся следующими соотношениями:

$$
C_{0}=2 \pi \varepsilon_{0} / \ln (D / d) ; \quad L_{0}=\left(\mu_{0} / 2 \pi\right) \ln (D / d),
$$

где $D$ и $d$ - внутренний диаметр цилиндрической части коаксиала и наружный диаметр центрального провода соответственно. Вводимые погонные параметры можно назвать полевыми, поскольку речь идёт о той энергии, которая запасена в электрических и магнитных полях. При этом не учитывается наличие кроме полевой ещё и кинетической индуктивности, обусловленной кинетической энергией движущихся зарядов. В реальных линиях передачи это оправдано, так как ввиду очень большой плотности носителей тока в проводниках их скорость мала, и поэтому полевая индуктивность всегда значительно больше кинетической.

При токе $I$, текущем по центральному проводнику, энергия, накопленная в удельной индуктивности, связана с погонной индуктивностью:

$$
W_{L}=L_{0} I^{2} / 2=\left(\mu_{0} /(4 \pi)\right) \ln (D / d) I^{2} .
$$

Пусть ток равномерно распределён по сечению центрального проводника. Тогда кинетическая энергия зарядов в проводнике единичной длины равна

$$
W_{k}=\pi d^{2} n m v^{2} / 8,
$$

где $n, m, v$ - плотность электронов, их масса и скорость соответственно. 
Если учесть, что $I=n e v \pi d^{2} / 4$, то можно записать

$$
W_{L}=\frac{1}{2} L_{0} I^{2}=\frac{\mu_{0}}{4 \pi} \ln \left(\frac{D}{d}\right) \frac{n^{2} e^{2} v^{2} \pi^{2} d^{4}}{16} .
$$

Получается, что случаю $W_{k} \geq W_{L}$ соответствует условие

$$
\frac{m}{n e^{2}} \geq \frac{\mu_{0}}{8} \ln \left(\frac{D}{d}\right) d^{2}
$$

где $L_{k}=m /\left(n e^{2}\right)$ - удельная кинетическая индуктивность зарядов.

Отсюда находим, что для кинетических пучков необходимо условие

$$
n \leq 8 m /\left(d^{2} e^{2} \mu_{0}\right) \text {. }
$$

Таким образом, чтобы поток был кинетическим, необходимо, чтобы удельная кинетическая индуктивность превышала погонную индуктивность, что выполняется при соблюдении приведенного условия. Из него можно оценить соответствующую плотность электронов в потоке.

Рассмотрим конкретный пример: $d=1$ мм, $\ln (D / d)=2$. Тогда имеем:

$$
n \leq \frac{8 m}{e^{2} \mu_{0} \ln (D / d) d^{2}} \approx 10^{20} \frac{1}{\mathcal{M}^{3}} .
$$

Такие плотности характерны электронным пучкам. Они значительно ниже плотности электронов в проводниках. Поэтому электронные пучки следует отнести к кинетическим потокам, в то время как электронные токи в проводниках относятся к потенциальным потокам. Поэтому для расчёта энергии, переносимой электромагнитными полями, пользуются вектором Пойнтинга, а для расчёта энергии, переносимой электронными пучками, используют кинетическую энергию отдельных зарядов. Это тем более правильно, когда речь идёт о расчёте энергии, переносимой ионными пучками, т.к. масса ионов во много раз превышает массу электронов.

Таким образом, причисление потоков зарядов к тому или другому виду зависит не только от плотности и диаметра пучка, но и от диаметра проводящей трубки, в которой он распространяется. Скорость распространения фронта потенциального пучка не превышает скорости света. Есть ли такие ограничения для чисто кинетических пучков? Чёткого ответа на этот вопрос пока нет. Массу электрона обычно связывают с его электрическими полями, и если при помощи внешней проводящей трубки ограничить эти поля, то масса электрона уменьшится, но это приведёт к уменьшению кинетической индуктивности, и пучок будет терять свои кинетические свойства. И только в том случае, если часть массы электрона не имеет электрического происхождения, есть надежда организовать чисто кинетический пучок электронов, скорость которого может превышать скорость света. Случай пучка протонов аналогичен. Но при помощи ядер дейтерия, содержащих нейтрон, у которого масса имеется, а электрических полей нет, можно получить чисто кинетические пучки и рассчитывать на возможность их разгона до сверхсветовых скоростей. Если выпустить такой пучок из ограничивающей трубки в свободное пространство, т.е. попытаться превратить его из кинетического в потенциальный, то можно получить че- 
ренковское излучение типа того, когда электронный поток попадает в среду, где фазовая скорость электромагнитной волны меньше его скорости.

\section{§ 14. Переходные процессы в отрезках длинных линий}

Фарадей экспериментально установил закон индукции, включая и выключая ток в соленоидах, или поддерживая в них постоянный ток и двигая по отношению к ним витки проволоки с подключённым гальванометром. Сегодня считается верным его представление о том, что если к соленоиду с индуктивностью $L$ подключён источник постоянного напряжения $U$, то ток во всех его витках нарастает по линейному закону

$$
I=U t / L,
$$

При такой интерпретации магнитное поле на всём протяжении соленоида нарастает синхронно. Но так ли это на самом деле?

Если линию на рис. 2 закоротить на расстоянии $z_{1}$ от ее начала, то её суммарная индуктивность составит $L_{\Sigma}=z_{1} L_{0}=z_{1}(a / b) \mu_{0}$. Если подключить к линии источник постоянного напряжения, в ней начнет распространяться волна напряжения $U$ и тока $I=U / Z$ (рис. 8). Напряжение на входе линии вырастает от нуля до своего номинального значения за время переходного процесса $\tau=z_{2} / c$, где $z_{2}$ - длина фронта волны, то есть переходного участка, к которому приложено напряжение источника питания и на котором заряды ускоряются от нулевой скорости до значений, необходимых для создания номинального тока в линии.

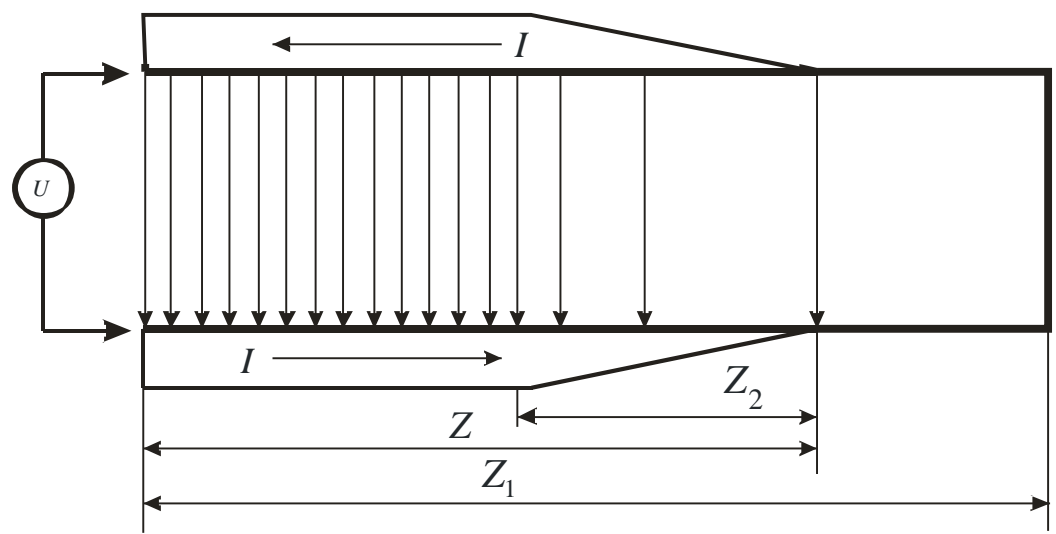

Рис. 8. Распространение волны тока и напряжения в длинной линии

Это время зависит от закона роста напряжения на входе линии после подключения к ней источника напряжения. Реально он может быть любым, но для простоты примем его линейным. Пусть время этого переходного процесса значительно меньше времени пробега фронта напряжения по ли- 
нии в одну сторону. Переходный процесс связан с инерционными свойствами устройства, подключающего источник напряжения к линии, или создаётся искусственно путём повышения напряжения по определённому закону. Предполагается, что $z_{1}$ значительно больше, чем $z_{2}$. Когда фронт напряжения $U$ появляется на перемычке в конце линии, на которой выполняется граничное условие $U=0$, возникает отраженная волна с напряжением $-U$, бегущая в обратном направлении. Так как ток в отраженной волне равен напряжению с отрицательным знаком, и двигается она в обратном направлении, то суммарный ток, создаваемый этой волной, равен $-(-U / Z)=U / Z$, т.е. он течёт в направлении падающей волны. Таким образом, отраженная волна, двигаясь в обратном направлении, оставляет после себя ток $2 U / Z$ и нулевое напряжение. Когда фронт напряжения возвратится к началу линии, он принесет с собой состояние удвоенного начального тока и нулевое напряжение. Источник снова пошлет в линию фронт напряжения $U$ и ток $U / Z$. Этот ток сложится с током $2 U / Z$, и суммарный ток в линии составит $3 U / Z$. Ток и далее будет нарастать ступеньками, добавляя каждый очередной раз к своему прежнему значению величину $2 U / Z$.

На рис. 9. этот процесс отображён во времени. За время

$$
T=z_{1} / c=z_{1} \sqrt{L_{0} C_{0}}=z_{1} \sqrt{\mu_{0} \varepsilon_{0}}
$$

фронт напряжения пробегает по линии в одну сторону от её начала до закороченного участка. Особенность процесса - скачкообразность отбора энергии от источника напряжения. На интервале времени от нуля до $2 T$ отбирается мощность $U^{2} / Z$, а на каждом последующем интервале времени длины $2 T-$ уже $2 U^{2} / Z$. При любой длине линии ток нарастает скачкообразно, а не линейно. При малой длине скачки следуют через малые промежутки времени, и зависимость тока от времени близка к линейной, что и характерно для элементов с сосредоточенными параметрами.

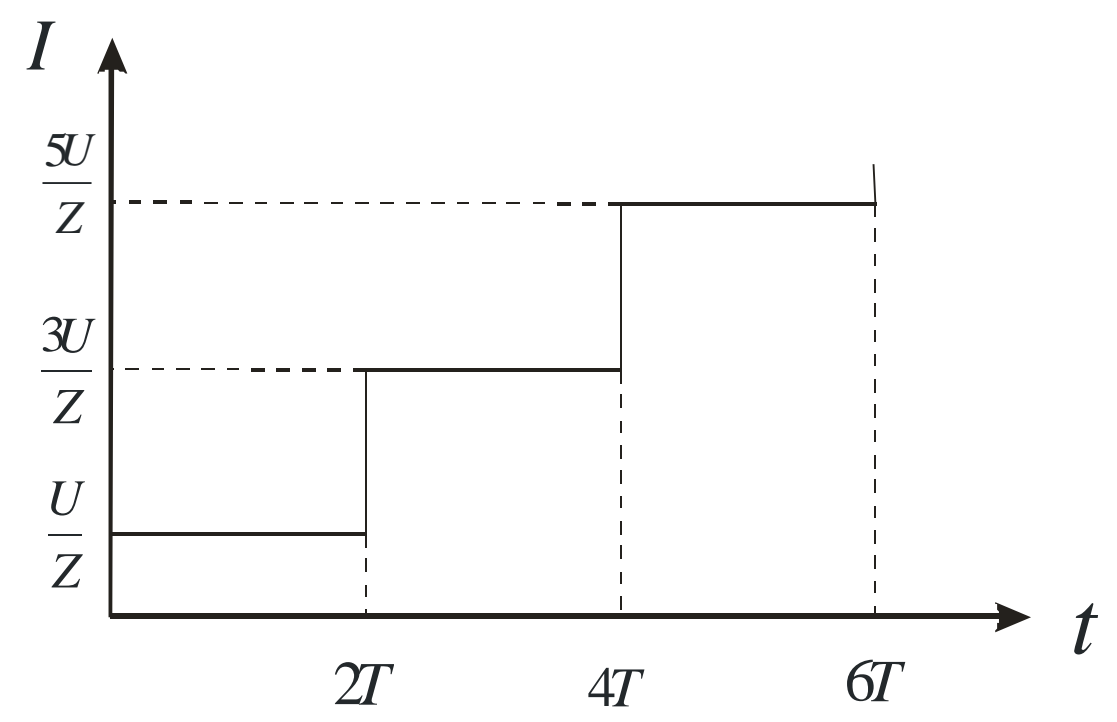

Рис. 9. Зависимости входного тока от времени для закороченной линии 
Магнитный поток изменяется линейно (рис. 10). Во время прямого хода длительностью $T$ до момента достижения волной закороченного участка поток увеличивается линейно до $\Phi_{B}=z_{1} U / c$. При обратном ходе фронта напряжения после отражения от закороченного участка до прихода обратно к источнику напряжения поток опять линейно возрастает уже до $\Phi_{B}=2 z_{1} U / c$. Таким образом, выполняется закон индукции $U=d \Phi_{B} / d t$.

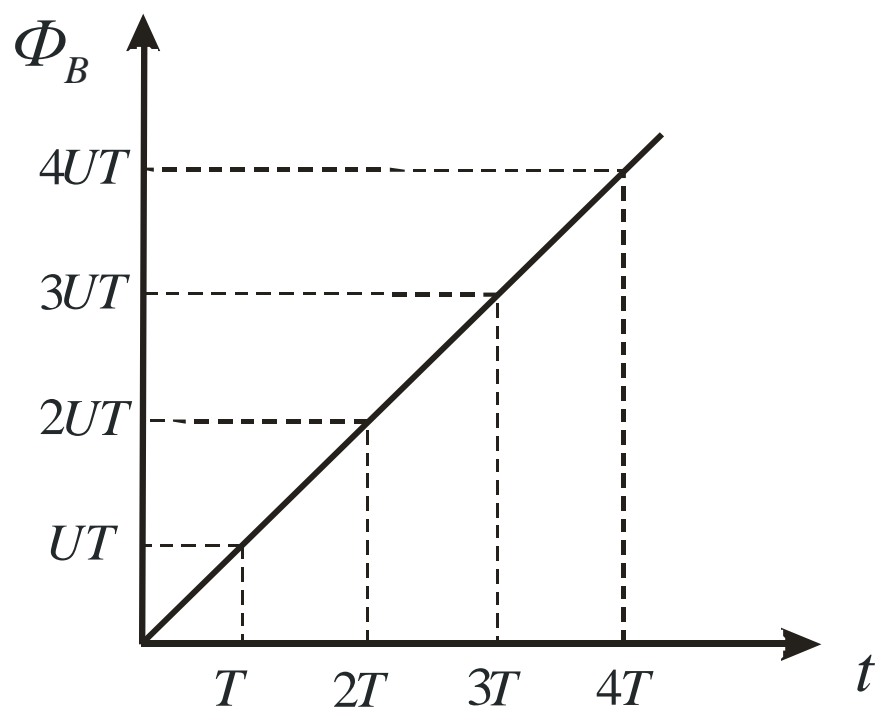

Рис. 10. Линейный рост магнитного потока для закороченной линии

Электрический поток в линии изменяется периодически, то возрастая, то убывая линейно (рис. 11). При движении волны в положительном направлении одновременно возрастает и магнитный, и электрический поток.

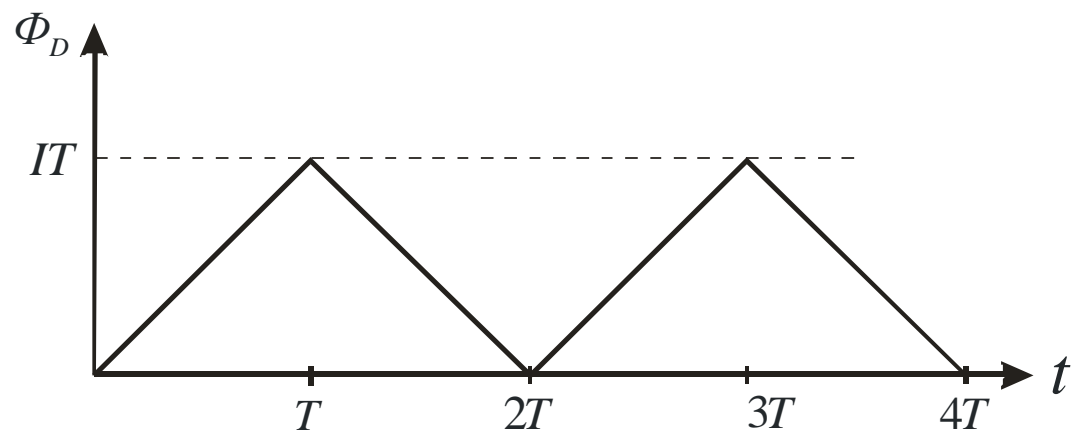

Рис. 11. Колебание электрического потока для закороченной линии

При этом, как в магнитном, так и в электрическом поле накопленная энергия возрастает. При обратном ходе волны электрическое поле слабеет, а его энергия переходит в магнитную энергию обратной волны тока. После достижения фронтом волны напряжения входа линии магнитное поле и ток в ней удваиваются, а электрическое поле исчезает. Далее цикл повторяется. Следовательно, росту магнитного потока в индуктивности закороченной длинной линии обязательно сопутствует периодическое изменение потока электрической индукции, в результате чего между плоскостями линии периодически возникает и исчезает электрическое поле. 
Если линия выполнена из сверхпроводника и не имеет потерь, то, заменив в определённый момент генератор напряжения сверхпроводящей перемычкой, можно заморозить ток в линии. Самый благоприятный момент для этого - отсутствие электрического поля в линии. Тогда в линии заморозится поток $\Phi_{B}=2 N z_{1} U / c$, соответствующий току $I=2 N U / Z$. Если произвести замену в момент, когда в линии находится фронт напряжения и какой-то её участок заполнен электрическими полями, то этот участок будет двигаться в линии, попеременно отражаясь то от одного, то от другого конца закороченной линии, пока не истратит свою энергию на излучение.

По этой причине в закороченной с обеих сторон линии может быть заморожено только целочисленное (квантованное) значение потока и тока в соответствии с приведенными соотношениями. Это явление является примером макроскопического квантования потока в макроскопических структуpax, имеющих определённые размеры. Такое же квантование потока происходит и в микроскопических структурах, которыми являются атомы.

Для выяснения того, действительно ли ток в соленоиде при подключении источника напряжения растёт линейно одновременно во всех витках, заменим верхнюю плоскость рассмотренной двухпроводной линии (рис. 12) длинным соленоидом. При подключении к линии источника ток в ней растёт так же. Погонная индуктивность линии теперь в основном определяется погонной индуктивностью соленоида, и скорость распространения волны тока и волны напряжения (напряжение теперь приложено между соленоидом и нижним проводником линии) меньше, чем в предыдущем случае.

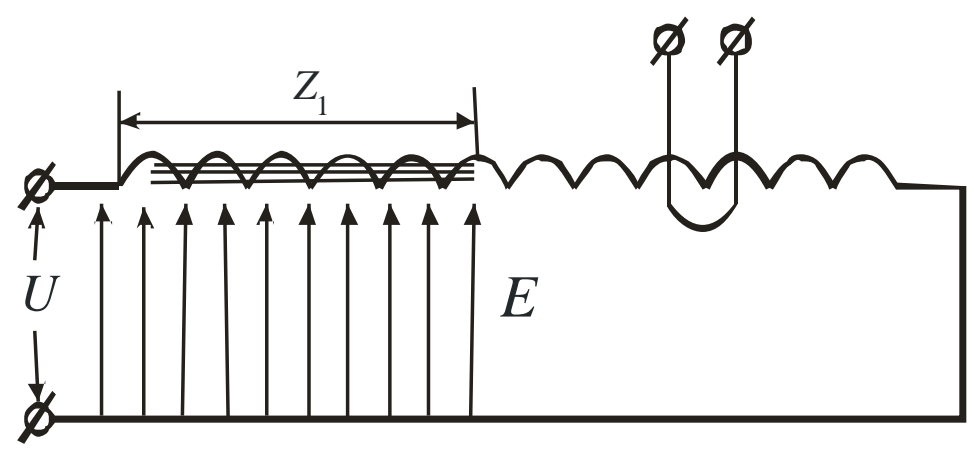

Рис. 12. Схема распространения полей в длинном соленоиде

Когда волна дойдет до точки $z_{1}$, магнитным полем заполнится часть соленоида между источником питания и точкой $z_{1}$. Когда волна дойдет до его конца, магнитным полем заполнится весь соленоид. При обратном ходе волны магнитное поле в соленоиде удвоится, и процесс начнется сначала.

Положим теперь, что соленоид в определенном месте охвачен витком. Этот процесс подобен механическому одеванию охватывающего витка на конец соленоида с той лишь разницей, что в данном случае магнитный поток, перемещаясь внутри соленоида, сам пронизывает охватывающий его виток. Причём скорость движения фронта магнитного потока при этом несоизмеримо больше, чем при механическом одевании. Но оба процесса 
имеют одинаковую природу. Этими процессами и объясняется явление взаимной индукции между соленоидом и охватывающим витком. При небольшой длине соленоида расстояние между импульсами невелико, поэтому они, сливаясь, образуют почти постоянное напряжение. Напряжение в витке будет индуцироваться только в момент пересечения магнитным потоком соленоида окрестностей поперечного сечения, охваченного витком. В этот момент в окрестностях охватывающего витка будет возникать, как векторный потенциал, так и магнитное поле. И, именно, пересечение охватывающего витка магнитными полями рассеяния (такого же, как и на конце соленоида с постоянным током) приводит к индуцированию в нём ЭДС. Этот момент будет наступать как при прямом, так и при обратном ходе волны, причем полярность импульсов напряжения, индуцируемых в витке, в обоих случаях будет одна и та же. Частота этих импульсов будет зависеть от длины соленоида, и будет тем больше, чем короче соленоид. Следовательно, среднее значение индуцированного напряжения будет расти с уменьшением длины соленоида, т.е. его количества витков, что и определяет коэффициент трансформации такого трансформатора, который равен отношению количества витков соленоида и охватывающей обмотки.

Рассмотрев процесс нарастания токов и полей в длинном соленоиде, вернёмся к вопросу о наличии циркуляции векторного потенциала вокруг него. Приведём существующую точку зрения, представленную в [4]. Векторный потенциал в пространстве вокруг соленоида равен

$$
A(r)=n I r_{0}^{2} /\left(2 \varepsilon_{0} c^{2} r\right),
$$

где $n$ - число витков, приходящееся на единицу длины соленоида, $I$ - ток, текущий через соленоид, $r_{0}$ - диаметр соленоида, $r$ - расстояние от оси соленоида до точки наблюдения. При этом полагается, что $r \geq r_{0}$.

Индуктивность соленоида зависит ещё от его длины $z_{0}$ :

$$
L=n^{2} \pi r_{0}^{2} z_{0} /\left(\varepsilon_{0} c^{2}\right) \text {. }
$$

Если к соленоиду с общим числом витков $N$ подключить источник постоянного напряжения $U$, то с учётом (14.1)-(14.3) получаем

$$
A(r, t)=U t /(2 \pi N r) \text {, }
$$

а поскольку

$$
E=-\mu_{0} \frac{\partial A}{\partial t},
$$

то напряжённость электрического поля в окрестности соленоида в момент подключения к нему источника постоянного напряжения составит

$$
E(r)=\mu_{0} U /(2 \pi N r) \text {. }
$$

Указанная напряжённость электрического поля в соответствии с рассматриваемой версией возникает в момент подключения к соленоиду источника питания мгновенно на всём его протяжении. Если у соленоида отсутствует сопротивление, то напряжённость электрического поля будет неизменной за весь период времени подключения к соленоиду источника постоянного напряжения. Какие здесь возникают противоречия? Во- 
первых, электрические поля обладают энергией, и возникать мгновенно не могут. Второе противоречие вытекает из первого и заключается в том, что, поскольку электрические поля обладают энергией, то эта энергия должна включаться в общую энергию, накопленную в соленоиде. Но при расчёте такой энергии учитываются только магнитные поля внутри соленоида.

Таким образом, сам процесс индукции электрических полей вокруг длинного соленоида происходит совсем не так, как это представлено в существующей литературе [4], когда считается, что циркуляция магнитного векторного потенциала на всём его протяжении возрастает одновременно, что и приводит к индукции ЭДС в охватывающем витке. Точка зрения о возникновении электрических полей индукции вокруг соленоида в том месте, где ротор векторного потенциала равен нулю, не соответствует действительности, а сам процесс формирования векторного потенциала снаружи соленоида и магнитных полей внутри него не соответствует существующим представлениям. Ротор векторного потенциала снаружи соленоида равен нулю, такое поле не обладает энергией, поэтому обнаружить его в статическом режиме невозможно. По этой причине эксперименты Аронова и Бома по обнаружению векторного потенциала снаружи намагниченного цилиндра следует считать ошибочными. 


\section{ЧАСТЬ II. НОВЫЕ ИДЕИ В КЛАССИЧЕСКОЙ ЭЛЕКТРОДИНАМИКЕ}

\section{ГЛАВА 4. НОВЫЕ ПОДХОДЫ И ОПРЕДЕЛЕНИЯ}

Новые идеи не возникают на пустом месте, их появлению предшествует накопление и осмысление ранее полученных результатов. Тот факт, что классическая электродинамика состоит по сути дела из двух, не связанных между собой частей, не может не вызывать беспокойства. То, что некоторые электродинамические эффекты не могут быть объяснены в рамках единой теории, тоже говорит о том, что классическую электродинамику пока нельзя считать завершенной физической теорией. В первой части книги показаны большая роль магнитного векторного потенциала в электродинамике и грубые нестыковки при его использовании для объяснения возникновения электрических полей вокруг длинного соленоида. Это ставит под сомнение и сам закон индукции Фарадея, поскольку векторный потенциал, магнитное поле и магнитный поток в соленоиде тесно связаны. Во времена Фарадея не были известны уравнения Максвелла и то, что электромагнитные поля распространяются с конечной скоростью в длинных линиях, а, следовательно, эти тесно связанные с процессами индукции эффекты тоже не могли быть учтены. Даже Максвелл, записав свои знаменитые уравнения, не мог экспериментально доказать существование электромагнитных волн. Сделал это впервые Герц, создав очень простым способом первый в мире СВЧ генератор [37], и при помощи этой методики получил выдающиеся результаты, показав, что электромагнитные волны могут распространяться не только в свободном пространстве, но и могут с конечной скоростью распространяться вдоль металлических проводников. Как было показано в предыдущих разделах, использование субстанциональной производной при записи законов индукции позволяет получить силу Лоренца из этих законов, а не постулировать её. В этом разделе мы попытаемся построить такую электродинамику, которая объединит её разрозненные части, укажем ту единую концептуальную основу и предложим тот математический аппарат, которые являются её фундаментом.

\section{§ 15. Динамические потенциалы и поля движущихся зарядов}

При своём распространении волна в длинной линии заполняется двумя видами энергии, которые могут быть определены через токи и напряжения или через электрические и магнитные поля в линии. Только после того, как волна заполнит электромагнитной энергией всё пространство между генератором и нагрузкой, в нагрузке начнёт выделяться энергия. За время задержки этого процесса генератор расходовал свою мощность на заполнение энергией участка линии между генератором и нагрузкой. Если начать удалять нагрузку от входа линии, то выделяемая на ней энергия уменьшится, т.к. часть энергии, расходуемой источником, уйдёт на заполнение энер- 
гией дополнительной длины линии, связанной с движением нагрузки [38]. Если нагрузка будет приближаться к источнику, то она будет получать дополнительную энергию за счёт сокращения её длины. Но если нагрузкой линии является активное сопротивление, то увеличение или уменьшение расходуемой в нём мощности может быть связано только с изменением напряжения на таком сопротивлении. Следовательно, движение наблюдателя относительно уже существующих в линии полей должно приводить к их изменению. Продуктивность такого подхода с применением преобразований Галилея и будет продемонстрирована в данной главе.

Поля, которые создаются в данной ИСО движущимися зарядами (например, магнитное поле вокруг движущихся зарядов) и движущимися источниками электромагнитных волн, назовём динамическими. В классической нерелятивистской электродинамике нет преобразований электрических и магнитных полей при переходе из одной ИСО в другую. Этот недостаток устраняет СТО, используя вместо преобразований Галилея преобразования Лоренца. При всей математической обоснованности такого подхода физическая сущность таких преобразований остаётся невыясненной [39].

В данном разделе будет сделана попытка найти именно физически обоснованные пути получения преобразований полей при переходе из одной ИСО в другую и выяснить, какие динамические потенциалы и поля генерируют движущиеся заряды. Первый шаг в этом направлении, продемонстрированный в $[33,40]$, - введение симметричных законов магнитоэлектрической и электромагнитной индукции, записываемых в виде [22,40-43]:

$$
\int\left[\mathbf{E}^{\prime} d \mathbf{l}^{\prime}=-\int \frac{\partial \mathbf{B}}{\partial t} d \mathbf{s}+\int[\mathbf{v} \times \mathbf{B}] d \mathbf{l}^{\prime} ; \quad \int \mathbf{H}^{\prime} d \mathbf{l}^{\prime}=\int \frac{\partial \mathbf{D}}{\partial t} d \mathbf{s}-\int\left[[\mathbf{v} \times \mathbf{D}] d \mathbf{l}^{\prime}\right.\right.
$$

или

$$
\operatorname{rot} \mathbf{E}^{\prime}=-\frac{\partial \mathbf{B}}{\partial t}+\operatorname{rot}[\mathbf{v} \times \mathbf{B}] ; \quad \operatorname{rot} \mathbf{H}^{\prime}=\frac{\partial \mathbf{D}}{d t}-\operatorname{rot}[\mathbf{v} \times \mathbf{D}] .
$$

Для постоянных полей эти соотношения имеют вид:

$$
\mathbf{E}^{\prime}=[\mathbf{v} \times \mathbf{B}] ; \quad \mathbf{H}^{\prime}=-[\mathbf{v} \times \mathbf{D}] .
$$

В соотношениях (15.1)-(15.3), основанных на преобразованиях Галилея, штрихованные и не штрихованные величины представляют поля и элементы в движущейся и неподвижной ИСО соответственно. Заметим, что преобразования (15.3) ранее получали только из преобразований Лоренца.

Соотношения (15.1)-(15.3), представляющие законы индукции, не дают информации о возникновении полей в исходной неподвижной ИСО. Они описывают только закономерности распространения и преобразования полей в случае движения по отношению к уже существующим полям.

Соотношения (15.3) свидетельствуют о том, что в случае относительного движения систем отсчета, между полями $\mathbf{E}$ и $\mathbf{H}$ существует перекрестная связь, т.е. движение в полях $\mathbf{H}$ приводит к появлению полей $\mathbf{E}$ и наоборот. Из этих соотношений вытекают дополнительные следствия, впервые рассмотренные в работе [9]. Если заряженный стержень имеет погонный заряд $g$, то его электрическое поле $E=g /(2 \pi \varepsilon r)$ убывает по закону $1 / r$, где $r$ - расстояние от центральной оси стержня до точки наблюдения. 
Если параллельно оси стержня в поле $E$ двигать со скоростью $\Delta v$ другую ИСО, то в ней появится дополнительное магнитное поле $\Delta H=\varepsilon E \Delta v$. Если теперь по отношению к уже движущейся ИСО двигать третью со скоростью $\Delta v$, то уже за счет движения в поле $\Delta H$ появится добавка к электрическому полю $\Delta E=\mu \varepsilon E(\Delta v)^{2}$ и т.д. Получается ряд, дающий величину электрического поля $E_{v}^{\prime}(r)$ в движущейся ИСО при достижении скорости $v=n \Delta v$, когда $\Delta v \rightarrow 0$, а $n \rightarrow \infty$. В конечном итоге, в движущейся ИСО величина динамического электрического поля окажется больше, чем в исходной, и зависящей от нормальной составляющей $v_{\perp}$ скорости заряда к вектору, соединяющему движущийся заряд и точку наблюдения:

$$
E^{\prime}\left(r, v_{\perp}\right)=\frac{g \operatorname{ch}\left(v_{\perp} / c\right)}{2 \pi \varepsilon r}=E \operatorname{ch}\left(v_{\perp} / c\right) .
$$

Электрическое поле одиночного заряда $e$ определяется соотношением

$$
E^{\prime}\left(r, v_{\perp}\right)=\frac{e \operatorname{ch}\left(v_{\perp} / c\right)}{4 \pi \varepsilon r^{2}} .
$$

Скалярный потенциал $\varphi^{\prime}\left(r, v_{\perp}\right)$ движущегося заряда назовём скалярновекторным (зависит не только от величины заряда, но и от скорости и направления его движения по отношению к точке наблюдения). Он выражается через скалярный потенциал $\varphi(r)$ неподвижного заряда равенством

$$
\varphi^{\prime}\left(r, v_{\perp}\right)=\frac{e \operatorname{ch}\left(v_{\perp} / c\right)}{4 \pi \varepsilon r}=\varphi(r) \operatorname{ch}\left(v_{\perp} / c\right) .
$$

Потенциал максимален в нормальном к движению заряда направлении. Он определяет даже электрические поля, индуцируемые ускоряемым зарядом.

Аналогично, для случая движения заряда в магнитном поле имеем:

$$
H^{\prime}\left(v_{\perp}\right)=H \operatorname{ch}\left(v_{\perp} / c\right) .
$$

где $v_{\perp}$ - скорость, нормальная к направлению магнитного поля.

Получим этот результат другим способом. Обозначим полевые переменные в неподвижной системе отсчёта без штриха, а в подвижной - со штрихом. В дифференциальной форме запишем формулы взаимной индукции электрического и магнитного полей в подвижной системе отсчёта:

$$
\begin{aligned}
& d H^{\prime}=\varepsilon E^{\prime} d v_{\perp} ; \\
& d E^{\prime}=\mu H^{\prime} d v_{\perp}
\end{aligned}
$$

или, иначе,

$$
\begin{aligned}
& d H^{\prime} / d v_{\perp}=\varepsilon E^{\prime} ; \\
& d E^{\prime} / d v_{\perp}=\mu H^{\prime},
\end{aligned}
$$

где (15.7) соответствует (15.5), а (15.8) соответствует (15.6).

Разделив уравнения (15.7) и (15.8) на $E$ и $H$, получим соответственно:

$$
\begin{aligned}
& \frac{d\left(H^{\prime} / E\right)}{d v_{\perp}}=\varepsilon \frac{E^{\prime}}{E} ; \\
& \frac{d\left(E^{\prime} / E\right)}{d v_{\perp}}=\mu \frac{H^{\prime}}{H} .
\end{aligned}
$$


Продифференцировав обе части (15.10), имеем:

$$
\frac{d^{2}\left(E^{\prime} / E\right)}{d^{2} v_{\perp}}=\mu \frac{d\left(H^{\prime} / E\right)}{d v_{\perp}} .
$$

Подставив (15.9) в (15.11), получим:

$$
\frac{d^{2}\left(E^{\prime} / E\right)}{d^{2} v_{\perp}}=\mu \varepsilon \frac{E^{\prime}}{E}
$$

Общим решением дифференциального уравнения (15.12) является функция

$$
E^{\prime} / E=C_{2} \operatorname{ch}\left(v_{\perp} / c\right)+C_{1} \operatorname{sh}\left(v_{\perp} / c\right)
$$

где $c$ - скорость света в среде, $C_{1}, C_{2}$ - произвольные постоянные.

Так как при $v_{\perp}=0$ должно быть выполнено $E^{\prime}=E$, то из (15.13) получим:

$$
C_{2}=1 \text {. }
$$

Подставив (15.14) в (15.13), окончательно имеем общее решение, в которое входит одна произвольная постоянная $C_{1}$ :

$$
E^{\prime} / E=\operatorname{ch}\left(v_{\perp} / c\right)+C_{1} \operatorname{sh}\left(v_{\perp} / c\right) .
$$

Выбирая $C_{1}=0$, получаем

$$
E^{\prime}=E \operatorname{ch}\left(v_{\perp} / c\right) .
$$

Применительно к электромагнитной волне, вводя параллельные $E_{\uparrow}, H_{\uparrow}$ и нормальные $E_{\perp}, H_{\perp}$ скорости ИСО компоненты полей, имеем [9]:

$$
\begin{aligned}
& \mathbf{E}_{\uparrow}^{\prime}=\mathbf{E}_{\uparrow} ; \quad \mathbf{E}_{\perp}^{\prime}=\mathbf{E}_{\perp} \operatorname{ch} \frac{v}{c}+\frac{Z_{0}}{v}\left[\mathbf{v} \times \mathbf{H}_{\perp}\right] \operatorname{sh} \frac{v}{c} ; \\
& \mathbf{H}_{\uparrow}^{\prime}=\mathbf{H}_{\uparrow} ; \quad \mathbf{H}_{\perp}^{\prime}=\mathbf{H}_{\perp} \operatorname{ch} \frac{v}{c}-\frac{1}{v Z_{0}}\left[\mathbf{v} \times \mathbf{E}_{\perp}\right] \operatorname{sh} \frac{v}{c},
\end{aligned}
$$

где $Z_{0}=\sqrt{\mu_{0} / \varepsilon_{0}}-$ импеданс свободного пространства, $c=1 / \sqrt{\mu_{0} \varepsilon_{0}}-$ скорость света.

Назовём преобразования полей (15.15) преобразованиями Менде.

Участник научного форума Движения за возрождение отечественной науки (http://www.forum.za-nauku.ru/index.php/board,6.0.html) Николай Александрович Дробышев привёл вывод (15.15) в матричной форме [44, 45] и показал, что вид преобразований определяется законом сложения скоростей (классический или релятивистский). Приведём его выкладки.

Рассмотрим совокупность ИСО таких, что ИСО К 1 движется со скоростью $\Delta v$ относительно ИСО К, ИСО К 2 движется с такой же скоростью $\Delta v$ относительно К 1 и т.д. Если модуль скорости $\Delta v$ мал (по сравнению со скоростью света с), то для поперечных составляющих полей в ИСО $\mathrm{K}_{1} \mathrm{~K}_{2}$, имеем:

$$
\begin{array}{ll}
\mathbf{E}_{1 \perp}=\mathbf{E}_{\perp}+\Delta \mathbf{v} \times \mathbf{B}_{\perp} & \mathbf{B}_{1 \perp}=\mathbf{B}_{\perp}-\Delta \mathbf{v} \times \mathbf{E}_{\perp} / c^{2} \\
\mathbf{E}_{2 \perp}=\mathbf{E}_{1 \perp}+\Delta \mathbf{v} \times \mathbf{B}_{1 \perp} & \mathbf{B}_{2 \perp}=\mathbf{B}_{1 \perp}-\Delta \mathbf{v} \times \mathbf{E}_{1 \perp} / c^{2}
\end{array}
$$

и т. д. При переходе к каждой следующей ИСО поля получают приращения $\Delta \mathbf{E}$ и $\Delta \mathbf{B}$ :

$$
\Delta \mathbf{E}=\Delta \mathbf{v} \times \mathbf{B}_{\perp}, \quad \Delta \mathbf{B}=-\Delta \mathbf{v} \times \mathbf{E}_{\perp} / c^{2},
$$


где поля $\mathbf{E}_{\perp}$ и $\mathbf{B}_{\perp}$ относятся к текущей ИСО. Направляя декартову ось $x$ вдоль $\Delta \mathbf{v}$, перепишем (15.17) в компонентах вектора

$$
\Delta E_{y}=-B_{z} \Delta v, \quad \Delta E=B_{y} \Delta v, \quad \Delta B_{y}=E_{z} \Delta v / c^{2} .
$$

Соотношение (15.18) можно представить в матричной форме

$$
\Delta U=A U \Delta v \quad\left(\begin{array}{lccc}
0 & 0 & 0 & -1 \\
0 & 0 & 1 & 0 \\
0 & 1 / c^{2} & 0 & 1 \\
-1 / c^{2} & 0 & 0 & 0
\end{array}\right) \quad U=\left(\begin{array}{l}
E_{y} \\
E_{z} \\
B_{y} \\
B_{z}
\end{array}\right) .
$$

Если предположить, что скорость системы суммируется по классическому закону сложения скоростей, т.е. скорость конечной ИСО $K^{\prime}=K_{N}$ относительно исходной $K$ есть $v=N \Delta v$, то получим матричную систему дифференциальных уравнений

$$
d U(v) / d v=A U(v)
$$

с независящей от скорости $v$ матрицей системы $A$. Решение системы выражается через матричную экспоненту $\exp (v A)$ :

$$
U^{\prime} \equiv U(v)=\exp (v A) U, \quad U=U(0) .
$$

Здесь $U$ - матрица-столбец полей в системе $K$, а $U^{\prime}$ - матрица-столбец полей в системе $K^{\prime}$. Подставляя (15.20) в систему (15.19), убеждаемся, что $U^{\prime}$ действительно является решением системы (15.19):

$$
\frac{d U(v)}{d v}=\frac{d[\exp (v A)]}{d v} U=A \exp (v A) U=A U(v) .
$$

Остаётся найти эту экспоненту разложением её в ряд:

$$
\exp (v a)=E+v A+\frac{1}{2 !} v^{2} A^{2}+\frac{1}{3 !} v^{3} A^{3}+\frac{1}{4 !} v^{4} A^{4}+\ldots,
$$

где $E$ - единичная матрица размером $4 \times 4$. Для этого удобно записать матрицу $A$ в блочной форме

$$
A=\left(\begin{array}{lr}
0 & -\alpha \\
\alpha / c^{2} & 0
\end{array}\right), \quad \alpha=\left(\begin{array}{ll}
0 & 1 \\
-1 & 0
\end{array}\right), \quad 0=\left(\begin{array}{ll}
0 & 0 \\
0 & 0
\end{array}\right) .
$$

Тогда

$$
\begin{array}{rlrl}
A^{2} & =\left(\begin{array}{cc}
-\alpha^{2} / c^{2} & 0 \\
0 & -\alpha / c^{2}
\end{array}\right), & A^{3}=\left(\begin{array}{cc}
0 & \alpha^{3} / c^{2} \\
-\alpha^{3} / c^{4} & 0
\end{array}\right), \\
A^{4}=\left(\begin{array}{cr}
\alpha^{4} / c^{4} & 0 \\
0 & \alpha^{4} / c^{4}
\end{array}\right), & A^{5}=\left(\begin{array}{ccc}
0 & -\alpha^{5} / c^{4} \\
\alpha^{5} / c^{6} & 0
\end{array}\right)
\end{array}
$$

и элементы матричной экспоненты имеют вид

$$
[\exp (v A)]_{11}=[\exp (v A)]_{22}=I-\frac{v^{2}}{2 ! c^{2}}+\frac{v^{4}}{4 ! c^{4}}-\ldots .
$$




$$
[\exp (v A)]_{21}=-c^{2}[\exp (v A)]_{12}=\frac{\alpha}{c}\left(\frac{v}{c} I-\frac{v^{3}}{3 ! c^{3}}+\frac{v^{5}}{5 ! c^{5}}-\ldots . .\right),
$$

где $I$ - единичная матрица $2 \times 2$. Нетрудно видеть, что $-\alpha^{2}=\alpha^{4}=-\alpha^{6}=\alpha^{8}=\ldots=I$, поэтому окончательно получаем

$$
\exp (v A)=\left(\begin{array}{ll}
I \operatorname{ch}(v / c) & -c \alpha \operatorname{sh}(v / c) \\
(\alpha \operatorname{sh}(v / c)) / c & I \operatorname{ch}(v / c)
\end{array}\right)=\left(\begin{array}{cccc}
\operatorname{ch}(v / c) & 0 & 0 & -c \operatorname{sh}(v / c) \\
0 & \operatorname{ch}(v / c) & c \operatorname{sh}(v / c) & 0 \\
0 & (\operatorname{ch}(v / c)) / c & \operatorname{ch}(v / c) & 0 \\
-(\operatorname{sh}(v / c)) / c & 0 & 0 & \operatorname{ch}(v / c)
\end{array}\right)
$$

Теперь возвращаемся к (15.20) и подставляя туда $\exp (v A)$, находим

$$
\begin{gathered}
E_{y}^{\prime}=E_{y} \operatorname{ch}(v / c)-c B_{z} \operatorname{sh}(v / c), \quad E_{z}^{\prime}=E_{z} \operatorname{ch}(v / c)+c B_{y} \operatorname{sh}(v / c), \\
B_{y}^{\prime}=B_{y} \operatorname{ch}(v / c)+\left(E_{z} / c\right) \operatorname{sh}(v / c), \quad B_{z}^{\prime}=B_{z} \operatorname{ch}(v / c)-\left(E_{y} / c\right) \operatorname{sh}(v / c)
\end{gathered}
$$

или в векторной записи

$$
\mathbf{E}_{\perp}^{\prime}=\mathbf{E}_{\perp} \operatorname{ch} \frac{v}{c}+\frac{v}{c} \mathbf{v} \times \mathbf{B}_{\perp} \operatorname{sh} \frac{v}{c}, \quad \mathbf{B}_{\perp}^{\prime}=\mathbf{B}_{\perp} \operatorname{ch} \frac{v}{c}-\frac{1}{v c} \mathbf{v} \times \mathbf{E}_{\perp} \operatorname{sh} \frac{v}{c} .
$$

Это и есть преобразования (15.15).

Возникает закономерный вопрос, почему они отличаются от соответствующих преобразований полей в СТО, ведь в ней при малых скоростях $\Delta \mathbf{v}$ имеют место исходные соотношения (15.16) и (15.17). Дело в том, что согласно релятивистскому закону сложения скоростей складываются не скорости ИСО, а их быстроты (https://ru.wikipedia.org/wiki/ Быстрота). Coгласно определению, быстрота вводится как

$$
\theta=c \operatorname{arth}(v / c) \text {. }
$$

Именно, если быстроты систем $K_{1}$ и $K, K_{2}$ и $K_{1}, K_{3}$ и $K_{2}$ и т.д. отличаются на $\Delta \theta$, то быстрота ИСО $K^{\prime}=K_{N}$ относительно $K$ есть $\theta=N \Delta \theta$. При малых скоростях $\Delta \theta \cong \Delta v$. Поэтому (15.17) можно записать так:

$$
\Delta \mathbf{E}=\Delta \theta \times \mathbf{B}_{\perp}, \quad \Delta \mathbf{B}=-\Delta \boldsymbol{\theta} \times \mathbf{E}_{\perp} / c^{2},
$$

где $\boldsymbol{\theta}=\theta \mathbf{v} / v$. Система (15.19) с учётом аддитивности быстроты, а не скорости, заменится системой уравнений

$$
d U(\theta) / d \theta=A U(\theta) .
$$

Таким образом, все выкладки будут аналогичны приведенным выше с той разницей, что вместо скоростей в выражениях будет фигурировать быстрота. В частности, формулы (15.21) принимают вид

$$
\mathbf{E}_{\perp}^{\prime}=\mathbf{E}_{\perp} \operatorname{ch} \frac{\theta}{c}+\frac{\theta}{c} \boldsymbol{\theta} \times \mathbf{B}_{\perp} \operatorname{sh} \frac{\theta}{c}, \quad \mathbf{B}_{\perp}^{\prime}=\mathbf{B}_{\perp} \operatorname{ch} \frac{\theta}{c}-\frac{1}{\theta c} \boldsymbol{\theta} \times \mathbf{E}_{\perp} \operatorname{sh} \frac{\theta}{c}
$$

или

$$
\mathbf{E}_{\perp}^{\prime}=\mathbf{E}_{\perp} \operatorname{ch} \frac{\theta}{c}+\frac{v}{c} \mathbf{v} \times \mathbf{B}_{\perp} \operatorname{sh} \frac{\theta}{c}, \quad \mathbf{B}_{\perp}^{\prime}=\mathbf{B}_{\perp} \operatorname{ch} \frac{\theta}{c}-\frac{1}{v c} \mathbf{v} \times \mathbf{E}_{\perp} \operatorname{sh} \frac{\theta}{c} .
$$

Так как 


$$
\operatorname{ch}(\theta / c)=1 / \sqrt{1-\operatorname{th}^{2}(\theta / c)}, \quad \operatorname{sh}(\theta / c)=\operatorname{th}(\theta / c) / \sqrt{1-\operatorname{th}^{2}(\theta / c)},
$$

то подстановка (15.22) в (15.23) приводит к хорошо известным релятивистским преобразованиям полей

$$
\mathbf{E}_{\perp}^{\prime}=\frac{1}{\sqrt{1-v^{2} / c^{2}}}\left(\mathbf{E}_{\perp}+\mathbf{v} \times \mathbf{B}_{\perp}\right), \quad \mathbf{B}_{\perp}^{\prime}=\frac{1}{\sqrt{1-v^{2} / c^{2}}}\left(\mathbf{B}_{\perp}-\frac{1}{c^{2}} \mathbf{v} \times \mathbf{E}_{\perp}\right) .
$$

При малых относительных скоростях преобразования (15.21) и (15.24) различаются, начиная с членов разложения порядка $v^{2} / c^{2}$.

Покажем, как при помощи соотношений (15.15) объясняется явление фазовой аберрации, не имеющее объяснений в рамках классической нерелятивистской электродинамики. Пусть имеются компоненты плоской волны $H_{z}$ и $E_{x}$, распространяющейся в направлении $y$, а штрихованная система движется в направлении $x$ со скоростью $v_{x}$. Тогда получим компоненты полей в штрихованной системе координат в соответствии с (15.15):

$$
E_{x}^{\prime}=E_{x}, \quad E_{y}^{\prime}=H_{z} \operatorname{sh} \frac{v_{x}}{c}, \quad H_{z}^{\prime}=H_{z} \operatorname{ch} \frac{v_{x}}{c} .
$$

Таким образом, имеется неоднородная волна, имеющая в направлении распространения компоненту $E_{v}^{\prime}$.

Запишем суммарное поле $E^{\prime}$ в движущейся ИСО:

$$
E^{\prime}=\sqrt{\left(E_{x}^{\prime}\right)^{2}+\left(E_{y}^{\prime}\right)^{2}}=E_{x} \operatorname{ch}\left(v_{x} / c\right) \text {. }
$$

Если вектор $\mathbf{H}^{\prime}$ по-прежнему ортогонален оси $y$, то вектор $\mathbf{E}^{\prime}$ теперь наклонен к ней на угол $\alpha$, определяемый соотношением:

$$
\alpha \cong \operatorname{sh}(v / c) \cong v / c .
$$

Это и есть фазовая аберрация. Именно на такой угол приходится наклонять телескоп по ходу движения Земли вокруг Солнца, чтобы наблюдать звезды, находящиеся в действительности в зените.

Вектор Пойнтинга теперь также направлен уже не по оси $y$, а, находясь в плоскости $x y$, наклонен к оси $y$ на угол, определяемый (15.26). Отношение абсолютных величин векторов $\mathbf{E}^{\prime}$ и $\mathbf{H}^{\prime}$ в обеих системах остались одинаковыми. Но абсолютная величина вектора Пойнтинга увеличилась. Даже поперечное к направлению распространения волны движение ИСО увеличивает ее энергию в этой ИСО. Физический смысл явления поясним следующей аналогией. Когда дождевые капли падают вертикально, то энергия у них одна. Но в инерциальной системе, двигающейся нормально к вектору их скорости, к этой скорости добавляется вектор скорости инерциальной системы. При этом абсолютная величина скорости капель в инерциальной системе будет равна корню квадратному из суммы квадратов указанных скоростей. Такой же результат дает нам и соотношение (15.25).

Если поляризация волны изменится, то результат останется прежним, так как преобразования по отношению к векторам $\mathbf{E}$ и $\mathbf{H}$ полностью симметричны. Единственным отличием будет то, что теперь получится волна, у которой появится в направлении распространения компонента $H_{v}^{\prime}$. 
Полученные волны имеют в направлении распространения дополнительные вектора электрического или магнитного поля, и в этом они похожи на $E$ и $H$ волны, распространяющиеся в волноводах. В данном случае возникает необычная волна, у которой фазовый фронт наклонен к вектору Пойнтинга на угол, определяемый соотношением (15.26). По сути дела, полученная волна является суперпозицией плоской волны с фазовой скоростью $c=1 / \sqrt{\mu \varepsilon}$ и дополнительной волны, ортогональной к направлению распространения плоской волны и имеющей бесконечную фазовую скорость.

Рассмотрим еще один случай, когда направление скорости движущейся системы совпадает с направлением распространения электромагнитной волны. Будем считать, что имеются компоненты плоской волны $E_{x}$ и $H_{z}$, а также компоненты скорости $\pm v_{y}$. Учитывая, что в этом случае выполняется соотношение $E_{x}= \pm Z_{0} H_{z}$, получаем, что амплитуды полей экспоненциально убывают или возрастают в зависимости от направления движения:

$$
\begin{aligned}
& E_{x}^{\prime}=E_{x}\left(\operatorname{ch} \frac{v_{y}}{c}-\operatorname{sh} \frac{v_{y}}{c}\right)=E_{x} \exp \left(\mp \frac{v_{y}}{c}\right), \\
& H_{z}^{\prime}=H_{z}\left(\operatorname{ch} \frac{v_{y}}{c}-\operatorname{sh} \frac{v_{y}}{c}\right)=H_{z} \exp \left(\mp \frac{v_{y}}{c}\right) .
\end{aligned}
$$

Волновому уравнению удовлетворяет волна напряжённости электрического (или магнитного) поля типа

$$
E(t, y)=E_{0} \sin (\omega t-k y),
$$

где $k=2 \pi / \lambda-$ волновое число.

При переходе в инерциальную систему, движущуюся со скоростью $\pm v_{y}$, наблюдается доплеровский сдвиг частоты.

Поперечный эффект Доплера, который обсуждается достаточно давно, до сих пор не нашел своего уверенного экспериментального подтверждения. Для наблюдения звезды из движущейся ИСО необходимо наклонять телескоп по ходу движения на угол (15.26). Звезда, наблюдаемая в зените, в действительности находится несколько позади по направлению движения. Ее угловое смещение от видимого положения при этом будет определяться тоже (15.26). Но это будет означать, что такая звезда по отношению к нам имеет радиальную составляющую скорости, определяемую соотношением

$$
v_{r}=v \sin \alpha .
$$

Для малых углов $\sin \alpha \cong \alpha, \alpha=v / c$, и доплеровский сдвиг частоты равен

$$
\omega_{d \perp}=\omega_{0} v^{2} / c^{2} .
$$

Данный результат численно совпадает с результатами СТО, но принципиально отличается тем, что в СТО поперечный эффект Доплера (15.27) считается реальным, а в данном случае это только кажущийся эффект. 


\section{§ 16. Уравнения Власова и лагранжиан движущегося заряда в концепции скалярно-векторного потенциала}

Идея Власова заключалась в самосогласованности электромагнитных полей в плазме и полей заряженных частиц. Этот принцип легко понять на примере объёмного резонатора. Его резонансную частоту определяют переменные электромагнитные поля, подчиняющиеся уравнениям Максвелла с граничными условиями. Если внутри резонатора появляется любой объект, в том числе свободные заряды, то резонансная частота меняется так, чтобы его электромагнитные поля были согласованы с полями, индуцированными посторонним объектом. Руководствуясь этим принципом, Власов изначально рассматривал систему общих уравнений плазмы, включающих три компоненты (электроны, ионы и нейтральные атомы), и записывал уравнение Больцмана для $s$-ой компоненты плазмы в виде [11]

$$
\frac{\partial f_{s}}{\partial t}+\operatorname{div}_{r} \vec{v} f_{s}+\frac{e_{s}}{m_{s}}(\vec{E}+[\vec{v} \times \vec{B}]) \operatorname{grad}_{v} f_{s}=\left[\frac{\partial f_{s}}{\partial t}\right]_{s 1}^{s t}+\left[\frac{\partial f_{s}}{\partial t}\right]_{s 2}^{s t}+\left[\frac{\partial f_{s}}{\partial t}\right]_{s 3}^{s t}
$$

где $f_{s}(\mathbf{r}, \mathbf{p}, t)$ - функция распределения.

Правая часть (16.1) представляет интегралы столкновений. Эта система уравнений включала также уравнения Максвелла и уравнения для заряда и тока, выраженные через функции распределения. Так как Власов интересовался только волновыми решениями, то он пренебрег вкладами интегралов столкновений, поскольку по его оценкам выходило, что частоты плазменных волн много больше частот парных столкновений частиц в плазме. То есть описание взаимодействия заряженных частиц в плазме посредством столкновений он заменил их самосогласованным полем для описания дальнодействующего потенциала. Вместо уравнения Больцмана Власов предложил использовать следующую систему уравнений для описания заряженных компонент плазмы (электронов с функцией распределения $f_{e}(\mathbf{r}, \mathbf{p}, t)$ и положительных ионов с функцией распределения $\left.f_{i}(\mathbf{r}, \mathbf{p}, t)\right)$ :

$$
\begin{aligned}
& \frac{\partial f_{e}}{\partial t}+\mathbf{v} \frac{\partial f_{e}}{\partial \vec{x}}-(e \mathbf{E}+e[\mathbf{v} \times \mathbf{B}]) \frac{\partial f_{e}}{\partial \vec{p}}=0, \quad \frac{\partial f_{i}}{\partial t}+\mathbf{v} \frac{\partial f_{i}}{\partial \mathbf{x}}+(e \mathbf{E}+e[\mathbf{v} \times \mathbf{B}]) \frac{\partial f_{i}}{\partial \mathbf{p}}=0, \\
& \operatorname{rot} \mathbf{H}=\mathbf{j}+\varepsilon \frac{\partial \mathbf{E}}{\partial t}, \quad \operatorname{rot} \mathbf{E}=-\frac{\partial \mathbf{B}}{d t}, \quad \operatorname{div} \mathbf{E}=\rho, \quad \operatorname{div} \mathbf{B}=0, \\
& \rho=e \int\left(f_{i}-f_{e}\right) d^{3} \mathbf{p}, \quad \vec{j}=e \int\left(f_{i}-f_{e}\right) \mathbf{v} d^{3} \mathbf{p} .
\end{aligned}
$$

В (16.2) члены в скобках в первых двух уравнениях представляют силу, действующую на движущийся заряд. Концепция скалярно-векторного по- 
тенциала позволяет найти её в системе координат движущегося заряда с учётом дальнодействующих сил, исключив магнитное поле $[10,18,22]$ :

$$
\mathbf{F}=-e \sum_{j} \frac{1}{4 \pi \varepsilon} \frac{g_{j}}{r_{j}^{2}} \operatorname{ch} \frac{v_{j \perp}}{c} \frac{\mathbf{r}_{j}}{r}
$$

где внешний заряд $g_{j}$ находится на расстоянии $r_{j}$ от заряда $e, v_{j \perp}-$ нормальная составляющая скорости заряда $g_{j}$ по отношению к заряду $e$.

Подставляя выражение для силы (16.3) в соотношения (16.2), получаем запись уравнений Власова в концепции скалярно-векторного потенциала:

$$
\begin{aligned}
& \frac{\partial f_{e}}{\partial t}+\mathbf{v} \frac{\partial f_{e}}{\partial \mathbf{x}}-\left(e \sum_{j} \frac{1}{4 \pi \varepsilon} \frac{g_{j}}{r_{j}^{2}} \operatorname{ch} \frac{v_{j \perp}}{c} \frac{\mathbf{r}_{j}}{r}\right) \frac{\partial f_{e}}{\partial \mathbf{p}}=0, \\
& \frac{\partial f_{i}}{\partial t}+\mathbf{v} \frac{\partial f_{i}}{\partial \mathbf{x}}+\left(e \sum_{j} \frac{1}{4 \pi \varepsilon} \frac{g_{j}}{r_{j}^{2}} \operatorname{ch} \frac{v_{j \perp}}{c} \frac{\mathbf{r}_{j}}{r}\right) \frac{\partial f_{i}}{\partial \mathbf{p}}=0, \\
& \operatorname{rot} \mathbf{H}=\mathbf{j}+\varepsilon \frac{\partial \mathbf{E}}{\partial t}, \quad \operatorname{rot} \mathbf{E}=-\frac{\partial \mathbf{B}}{d t}, \quad \operatorname{div} \mathbf{E}=\rho, \quad \operatorname{div} \mathbf{B}=0, \\
& \rho=e \int\left(f_{i}-f_{e}\right) d^{3} \mathbf{p}, \quad \mathbf{j}=e \int\left(f_{i}-f_{e}\right) \mathbf{v} d^{3} \mathbf{p} .
\end{aligned}
$$

В настоящее время уравнения Власова являются основными уравнениями электродинамики плазмы, в которых электромагнитные поля самосогласованы с полями зарядов, представляющих плазму. В эти уравнения входит сила Лоренца, которая в концепции скалярно-векторного потенциала может быть выражена через свойства заряженных частиц, окружающих точку наблюдения. Такой подход в полной мере реализует идею дальнодействия кулоновских полей - основу уравнений Власова.

В механике функция Лагранжа (лагранжиан) - разность зависящей от скорости кинетической и зависящей от координат потенциальной энергии:

$$
L=W_{k}(t)-W_{p}(t) .
$$

Его интеграл по времени называется действием:

$$
S=\int_{t_{1}}^{t_{2}} L\left(x_{i}, v_{i}\right) d t .
$$

Принцип наименьшего действия лагранжева формализма определяет движение консервативной системы по пути с минимальным действием.

В электродинамике лагранжиан релятивистского заряда равен (в СИ) [4]:

$$
L=-m c^{2} \sqrt{1-v^{2} / c^{2}}-q\left(\varphi+\mu_{0}\left(\mathbf{v} \mathbf{A}_{H}\right)\right),
$$

где $q, m$ и $\mathbf{v}$ - заряд, его масса и скорость, $\varphi, \mathbf{A}_{H}$ - скалярный и векторный потенциалы внешних электрического и магнитного полей.

Для нерелятивистских скоростей (16.4) примет вид:

$$
L=m v^{2} / 2-q\left(\varphi+\mu_{0}\left(\mathbf{v A}_{H}\right)\right) .
$$

Здесь заряд взаимодействует не с движущимися зарядами, влияя на их движение, а с векторным потенциалом, как самостоятельной субстанцией, 
нарушая консерватизм системы. В [15] на стр. 279 делается вывод: «Поэтому даже в релятивистском приближении функция Лагранжа в электромагнитном поле не может быть представлена в виде разности кинетической и потенциальной энергии» (конец цитаты), т.е. в (16.4) член, содержащий скалярное произведение скорости заряда и векторного потенциала, нельзя однозначно отнести ни к кинетической, ни к потенциальной энергии. Ландау в [14] фактически тоже разделяет эту точку зрения, делая следующее замечание при введении понятий действия и функции Лагранжа в параграфе 16: «Следующие ниже утверждения нужно рассматривать в значительной степени как результат опытных данных. Вид действия для частицы в электромагнитном поле не может быть установлен на основе только общих соображений, таких как требование релятивистской инвариантности. (последнее допускало бы в действии также и член вида интеграл от Ads, где A - скалярная функция)» (конец цитаты). Однако далее не приводятся ни опытные данные, ни теоретическое обоснование введения лагранжиана в виде (16.4). Зато возникают теоретические проблемы. Так, автор включает потенциальную часть (скалярное произведение скорости и векторного потенциала) в обобщённый импульс, а затем для нахождения силы дифференцирует лагранжиан по координате, вычисляя градиент (см. соотношение после (18.1) в [14]). Но нахождение градиента от указанного произведения означает признание его потенциальности.

В механике, умножая импульс на скорость, получают энергию. В электродинамике кроме соответствующего кинетического импульса, обусловленного массивностью носителей зарядов, вводится обобщенный импульс

$$
\mathbf{P}=m \mathbf{v}+q \mathbf{A}
$$

в виде суммы кинетического импульса и произведения заряда на векторный потенциал внешнего магнитного поля. Даже при незначительных магнитных полях эта добавка значительно превосходит сам кинетический импульс. Если обобщённый импульс скалярно умножить на скорость

$$
\mathbf{v P}=m(\mathbf{v})^{2}+q \mathbf{v A},
$$

то кинетический импульс даст кинетическую энергию. Скалярное произведение скорости и векторного потенциала также даст энергию, но не кинетическую, а потенциальную. Получается противоречивое смешение кинетической и потенциальной энергий в составе энергии движущегося заряда, что и характеризует возникающую теоретическую проблему.

Входящий в (16.4) векторный потенциал связан с движением сторонних зарядов. Их скорости подвержены влиянию изменения скорости данного заряда, на что затрачивается его энергия. Для обеспечения консерватизма системы необходим учет энергии взаимодействия данного движущегося заряда со всеми сторонними зарядами, в том числе с теми, от которых зависит векторный потенциал. Это становится возможным при использовании концепции скалярно-векторного потенциала [39-40, 42,43].

Скалярный потенциал совокупности сторонних зарядов в точке нахождения данного заряда равен сумме их скалярных потенциалов: 


$$
\varphi^{\prime}(r)=\sum_{j} \varphi\left(r_{j}\right) \operatorname{ch} \frac{v_{j \perp}}{c}=\sum_{j} \frac{1}{4 \pi \varepsilon} \frac{g_{j}}{r_{j}} \operatorname{ch} \frac{v_{j \perp}}{c}
$$

Потенциальная часть лагранжиана заряда $e$ равна:

$$
L=-e \sum_{j} \frac{1}{4 \pi \varepsilon} \frac{g_{j}}{r_{j}} \operatorname{ch} \frac{v_{j \perp}}{c} .
$$

К ней добавляется кинетическая часть, совпадающая в случае малых скоростей с чисто кинетической частью лагранжиана (16.5):

$$
L=\frac{m v^{2}}{2}-e \sum_{j} \frac{1}{4 \pi \varepsilon} \frac{g_{j}}{r_{j}} \operatorname{ch} \frac{v_{j \perp}}{c} .
$$

При этом величины $v_{j \perp}$ имеют смысл относительных скоростей сторонних зарядов по отношению к данному заряду.

Предложенный подход удовлетворяет полному консерватизму системы, поскольку учитывает все взаимодействия данного заряда с окружающими его сторонними зарядами. Соотношения (16.4) и (16.5) верны только для случая, когда заряд настолько мал, что не влияет на поля, в которых движется [13]. Запись лагранжиана в виде (16.6) исключает такое ограничение.

\section{$\S 17$. Транскоординатная электродинамика в пространственно-временном гиперконтинууме и преобразования Менде}

В классической механике динамика материальной точки описывается дифференциальными уравнениями для ее радиус-вектора, использующими обычную производную второго порядка по времени. Именно ее использование обеспечивает Галилей-инвариантность уравнений. Если соединить множество массивных материальных точек невесомыми упругими нитями в единую струну, то ее колебание будет описываться Галилейинвариантной системой дифференциальных уравнений. Но предельный переход (число материальных точек стремится к бесконечности, а их массы и длины отдельных нитей - к нулю) приводит к одномерному волновому уравнению (уравнение колебаний струны), не инвариантному относительно преобразований Галилея, зато инвариантному относительно группы псевдоортогональных преобразований (сохраняющих псевдоевклидову метрику гиперболических поворотов). Виновник странной и неожиданной метаморфозы при переходе от «материально-точечной механики к сплошной среде - этот предельный переход с подменой обычной производной на частную, который, вообще говоря, аналитически законен [46], но сужает область физической применимости уравнения. Реальный волновой процесс механических колебаний струны остается Галилей-инвариантным, но его уравнение уже лишено математических средств описания перехода от одной инерциальной системы отсчета к другой, а всецело привязывает процесс к одной конкретной системе отсчета, закрепляя в ней концы струны. 
Так классико-полевая естественнонаучная парадигма вскрыла фундаментальное противоречие между непрерывностью и дискретностью [47, 48], не преодоленное до сих пор, но приведшее к торжеству в теоретической физике сомнительного принципа геометризации [49].

Открытие волнового уравнения в механике не привело к пересмотру представлений о пространстве и времени, но к этому привело открытие того же самого уравнения в электродинамике. В теории относительности соответствующая группа псевдоортогональных преобразований для электромагнитных волн в вакууме (преобразования Лоренца) получила статус подгруппы движения метрики единого мирового физического пространства-времени. Но возникает сомнение в оправданности использования традиционных уравнений электродинамики, в частности, волнового, для адекватного извлечения из них представлений о пространстве и времени. Легко предположить, что эти уравнения, используя частные производные полевых функций по времени, подобно уравнению механических колебаний, попросту лишены математических средств адекватного описания перехода от одной инерциальной системы отсчета к другой и так же всецело привязывают процесс к одной конкретной системе отсчета. Возникает вопрос возможности подходящего уточнения или обобщения уравнений электродинамики, начиная с уравнений индукции электрического поля магнитным и магнитного - электрическим. Обстоятельное рассмотрение данного вопроса в [10] привело к появлению идеи о том, что такое совершенствование электродинамики должно предполагать существование зависимости электромагнитного поля от скорости движения наблюдателя, обусловленной не геометрией пространства-времени, а физической природой поля.

В теории относительности электромагнитное поле тоже зависит от скорости движения наблюдателя, но только опосредованно через зависимость от нее интервалов времени и пространственных расстояний (преобразования Лоренца), следствием чего оказывается релятивистская инвариантность электрического заряда. Более фундаментальная же (непосредственная) зависимость поля от скорости сопряжена с наличием такой зависимости даже абсолютной величины электрического заряда. До последнего времени такая неинвариантность заряда подтверждалась лишь косвенными эмпирическими данными - появление электрического потенциала на сверхпроводящих обмотках и торах при введении в них постоянного тока, а также в наблюдении электрического импульса ядерных взрывов [50].

Так, 9 июля 1962 года при взрыве в космосе над Тихим океаном водородной бомбы с тротиловым эквивалентом 1,4 Мт по программе США «Starfish» напряженность электрических полей превзошла предсказанные нобелевским лауреатом Бете Х.А. в 1000 раз. При взрыве в СССР осенью 1962 г. термоядерного заряда в космосе по программе «Программа К», радиосвязь и радарные установки были блокированы на расстоянии до 1000 км примерно аналогично случаю вышеуказанного американского взрыва. Было обнаружено, что регистрация последствий космического ядерного взрыва возможна на больших (до 10 тысяч километров) расстояниях от места взрыва. Электрические поля импульса привели к большим наводкам на 
силовой кабель в свинцовой оболочке, закопанный на глубину 1 м, соединяющий электростанцию в Акмоле с Алма-Атой. Наводки были настолько велики, что автоматика отключила кабель от электростанции.

Однако 2015 год ознаменовался получением уже прямого экспериментального подтверждения этого феномена в результате обнаружения и исследования импульса электрического поля, возникающего при разогреве плазмы в результате разряда через разрядники конденсаторов большой ёмкости [50]. Оказалось, что в процессе разогрева плазмы при равном количестве в ней электронов и положительных ионов в ней образуется унитарный отрицательный заряд свободных электронов, не скомпенсированный более медленными положительными ионами.

Этот факт противоречит не только классическим, но и релятивистским преобразованиям электромагнитного поля при переходе от одной инерциальной системы отсчета к другой, свидетельствуя о несовершенстве не только классических, но и релятивистских представлений о пространстве и времени. Идея о том, что перспективная электродинамика должна предполагать зависимость электромагнитного поля от скорости движения наблюдателя, обусловленную не геометрией пространства-времени, а физической природой поля, не предполагающей инвариантность электрического заряда, развивалась в ряде работ Менде Ф.Ф., начиная с [10]. В этих работах, в частности $[10,50]$, приведено обоснование введения в электродинамику вместо классических и релятивистских новых преобразований электромагнитного поля, получивших название преобразований Менде.

Однако последовательное развитие такой радикальной идеи, как неинвариантность заряда, требует глубокого пересмотра математического аппарата электродинамики, призванного к созданию математических средств более адекватного описания перехода от одной инерциальной системы отсчета к другой. Подход к именно такому развитию математического аппарата электродинамики был предложен Дубровиным А.С. в [51]. Этот подход лежит в рамках очередного пересмотра представлений о пространстве и времени с отказом от релятивистских и переходом к новым представлениям, которые мы называем гиперконтинуальными.

Понятие пространственно-временного гиперконтинуума введено в [52] в результате совместного изучения алгебраической и геометрической структур коммутативных алгебр с единицей, элементами которых являются функции синусоидальных волн. Гипотеза гиперконтинуума (об иерархической гиперконтинуальной структуре мирового физического пространствавремени) - отправная точка научных исследований в направлении обобщения представлений о структуре пространства и времени в русле перехода от современной квантовой научной парадигмы к новой системной, одновременно конструктивно соединяющей в своих рамках непрерывность и дискретность, динамичность и статичность, глобальность и локальность $[47,48,53]$. Иерархичность гиперконтинуума ограничивает применимость общепринятого принципа геометризации в физике и связанных с ним идей симметрии в геометрии за счет введения в теоретическую физику идей иерархичности $[49,54]$, эффективность которых апробирована нами при 
создании эталонной модели защищенной автоматизированной системы (ЭМЗАС) и математического аппарата ЭМЗАС-сетей [55].

В [51] предложен новый подход к развитию математического аппарата электродинамики в направлении более адекватного описания перехода от одной инерциальной системы отсчета к другой на основе гиперконтинуальных представлений о пространстве и времени за счет совершенствования дифференциального исчисления полевых функций в предположении их зависимости от скорости движения наблюдателя. Опишем этот новый подход более подробно в соответствии с [51].

Рассмотрим две инерциальные системы отсчета с единым для них временем $t \in \mathbb{R}$. Одну из них (с системой прямоугольных декартовых пространственных координат $O X Y Z$ ) назовем лабораторной (не штрихованной) и будем интерпретировать ее, как относительно неподвижную. Вторую (с системой прямоугольных декартовых пространственных координат $O^{\prime} X^{\prime} Y^{\prime} Z^{\prime}$ ) назовем субстанциональной (штрихованной) и будем интерпретировать ее, как связанную с некой движущейся реальной или воображаемой средой. Примем, что при $t=0$ системы пространственных координат обеих систем отсчета совпадают. Введем индексы $\alpha=\overline{1,3}, \beta=\overline{1,3}$. Координаты по осям $O X, O Y, O Z$ и $O^{\prime} X^{\prime}, O^{\prime} Y^{\prime}, O^{\prime} Z^{\prime}$ будем задавать переменными $x^{\alpha}$ и $x^{\prime \alpha}$ соответственно. Орты по осям $O X$ и $O^{\prime} X^{\prime}$, осям $O Y$ и $O^{\prime} Y^{\prime}$, осям $O Z$ и $O^{\prime} Z^{\prime}$ обозначим через $\mathbf{e}_{\beta}=\left(e_{\beta}^{\alpha}\right)$, причем $e_{\beta}^{\alpha}=\delta_{\alpha \beta}$, где $\delta_{\alpha \beta}$ - символ Кронекера. Через $\mathbf{v}=\left(v^{\alpha}\right)$ и $v$ обозначим вектор скорости движения субстанциональной системы отсчета относительно лабораторной и модуль этого вектора. Направляя орт $\mathbf{e}_{1}$ вдоль $\mathbf{v}$, имеем: $\mathbf{v}=v \mathbf{e}_{1}=\left(v^{\alpha}\right)$, $v^{\alpha}=v \delta_{\alpha 1}$. Событие в данных двух системах отсчета имеет вид $\mathbf{x}=(\mathbf{r}, t)=\left(x^{\alpha}, t\right) ; \quad \mathbf{x}^{\prime}=\left(\mathbf{r}^{\prime}, t\right)=\left(x^{\prime \alpha}, t\right), \quad$ где $\quad \mathbf{r}=\left(x^{\alpha}\right), \quad \mathbf{r}^{\prime}=\left(x^{\prime \alpha}\right)-$ радиусвекторы. Будем считать, что физическая эквивалентность событий $\mathbf{x}$ и $\mathbf{x}^{\prime}$ означает справедливость преобразования Галилея

$$
\mathbf{r}=\mathbf{r}^{\prime}+t \mathbf{v}
$$

или, иначе, заменяя векторное представление на компонентное,

$$
x^{\alpha}=x^{\prime \alpha}+t v \delta_{\alpha 1} .
$$

Классическое физическое поле описывается в лабораторной и субстанциональной системах отсчета своими полевыми функциями $\Phi(\mathbf{r}, t)$ и $\Phi^{\prime}\left(\mathbf{v}, \mathbf{r}^{\prime}, t\right)$, причем $\Phi^{\prime}\left(\mathbf{0}, \mathbf{r}^{\prime}, t\right)=\Phi\left(\mathbf{r}^{\prime}, t\right)$, а равенство $\mathbf{v}=0$ означает $v^{\alpha}=0$. Их значения называют полевыми переменными. Для полей разной физической природы могут подходить разные математические представления полевых функций, так что полевые переменные могут быть, например, скалярными или векторными с вещественными или комплексными значениями самих переменных или их векторных компонент. Если в роли такого поля выступает электрическое поле, то в данной роли могут выступать 
функции его напряженности $\mathbf{E}=\Phi(\mathbf{r}, t), \mathbf{E}^{\prime}=\Phi^{\prime}\left(\mathbf{v}, \mathbf{r}^{\prime}, t\right)$, а в случае магнитного поля имеем функции магнитной индукции $\mathbf{B}=\Phi(\mathbf{r}, t), \mathbf{B}^{\prime}=\Phi^{\prime}\left(\mathbf{v}, \mathbf{r}^{\prime}, t\right)$.

В классической нерелятивистской теории поля постулируется равенство

$$
\Phi\left(\mathbf{r}^{\prime}+t \mathbf{v}, t\right)=\Phi^{\prime}\left(\mathbf{v}, \mathbf{r}^{\prime}, t\right),
$$

математически выражающее физическую концепцию инвариантности поля относительно скорости движения наблюдателя.

В теории относительности (17.3) уже не выполняется, а вместо преобразований Галилея используются преобразования Лоренца. Но эта не инвариантность поля не имеет фундаментальной, не связанной с геометрией пространства-времени физической природы, а оказывается просто следствием эффектов сокращения длин и замедления времени в движущихся системах отсчета. Предлагаемые нами гиперконтинуальные представления о пространстве и времени [51] предусматривают широкие возможности инвариантности тех или иных физических процессов относительно тех или иных групп преобразований координат при том, что особую роль в пространственно-временном гиперконтинууме играют преобразования Галилея (17.1), так как они при этом трактуются, как уровневые преобразования Лоренца бесконечно высокого уровня и, тем самым, позволяют единым образом синхронизировать все события во всех отдельных континуумах, иерархически структурирующихся в единый гиперконтинуум. Естественно считать, что в гиперконтинууме поле также не инвариантно относительно скорости движения наблюдателя, но объяснять это уже фундаментальными свойствами поля, не связанными с геометрией отдельных континуумов.

Возникает вопрос о возможных вариантах полного дифференцирования по времени полевой функции в лабораторной системе отсчета $\Phi(\mathbf{r}, t)$, производимого в зависимости от субстанциональной системы отсчета. В гидроаэромеханике и классической механике широко используется производная Лагранжа (субстанциональная производная), имеющая те же аргументы, что и исходная полевая функция:

$$
\frac{d \Phi(\mathbf{r}, t)}{d t}=\frac{d \Phi\left(\mathbf{r}^{\prime}+t \mathbf{v}, t\right)}{d t}=\lim _{\Delta t \rightarrow 0} \frac{\Phi\left(\mathbf{r}^{\prime}+(t+\Delta t) \mathbf{v}, t+\Delta t\right)-\Phi\left(\mathbf{r}^{\prime}+t \mathbf{v}, t\right)}{\Delta t} .
$$

Но можно рассматривать также производную (назовем ее производной Галилея), аргументы которой будут совпадать с аргументами полевой функции уже не в лабораторной, а в субстанциональной системе отсчета:

$$
\frac{\partial^{\prime} \Phi}{\partial t}\left(\mathbf{v}, \mathbf{r}^{\prime}, t\right)=\frac{d \Phi\left(\mathbf{r}^{\prime}+t \mathbf{v}, t\right)}{d t}=\lim _{\Delta t \rightarrow 0} \frac{\Phi\left(\mathbf{r}^{\prime}+(t+\Delta t) \mathbf{v}, t+\Delta t\right)-\Phi\left(\mathbf{r}^{\prime}+t \mathbf{v}, t\right)}{\Delta t} .
$$

Если аргументы производных Лагранжа и Галилея связаны равенством (17.1), то их соответствующие значения равны и разлагаются в одну и ту же сумму частной по времени и конвективной производных полевой функции в лабораторной системе отсчета:

$$
\frac{\partial^{\prime} \Phi}{\partial t}\left(\mathbf{v}, \mathbf{r}^{\prime}, t\right)=\frac{d \Phi(\mathbf{r}, t)}{d t}=\frac{\partial \Phi\left(\mathbf{r}^{\prime}+t \mathbf{v}, t\right)}{\partial t}+(\mathbf{v} \cdot \nabla) \Phi\left(\mathbf{r}^{\prime}+t \mathbf{v}, t\right)
$$


Поясним различие физического смысла лагранжевой и галилеевой производных полевой функции. Производная Лагранжа (17.4) есть полная производная по времени функции поля в лабораторной системе отсчета, измеряемого в точке пространства, которая в лабораторной системе отсчета в момент времени $t$ имеет радиус-вектор $\mathbf{r}$, определяемый равенством (17.1). А производная Галилея (17.5) есть полная производная по времени функции поля в лабораторной системе отсчета, измеряемого в точке пространства, которая в субстанциональной системе отсчета имеет радиусвектор $\mathbf{r}^{\prime}$. Понятия лагранжевой и галилеевой производных (17.4-17.6) естественным образом обобщаются на случай высших порядков $(n=\overline{1, \infty})$ :

$$
\begin{gathered}
\frac{d^{1} \Phi(\mathbf{r}, t)}{d t^{1}}=\frac{d \Phi(\mathbf{r}, t)}{d t} ; \frac{d^{n+1} \Phi(\mathbf{r}, t)}{d t^{n+1}}=\frac{d}{d t} \frac{d^{n} \Phi(\mathbf{r}, t)}{d t^{n}} ; \\
\frac{\partial^{\prime 1} \Phi}{\partial t^{1}}\left(\mathbf{v}, \mathbf{r}^{\prime}, t\right)=\frac{\partial^{\prime} \Phi}{\partial t}\left(\mathbf{v}, \mathbf{r}^{\prime}, t\right) ; \frac{\partial^{\prime n} \Phi}{\partial t^{n}}\left(\mathbf{v}, \mathbf{r}^{\prime}, t\right)=\frac{d^{n} \Phi(\mathbf{r}, t)}{d t^{n}}
\end{gathered}
$$

В рамках концепции инвариантности поля относительно скорости движения наблюдателя, то есть при условии выполнения (17.3), имеем:

$$
\frac{\partial^{\prime} \Phi}{\partial t}\left(\mathbf{v}, \mathbf{r}^{\prime}, t\right)=\frac{d \Phi\left(\mathbf{r}^{\prime}+t \mathbf{v}, t\right)}{d t}=\frac{d \Phi^{\prime}\left(\mathbf{v}, \mathbf{r}^{\prime}, t\right)}{d t}=\frac{\partial \Phi^{\prime}\left(\mathbf{v}, \mathbf{r}^{\prime}, t\right)}{\partial t},
$$

то есть галилеева производная функции поля в лабораторной системе отсчета неотличима от частной производной по времени функции поля в субстанциональной системе отсчета. Поэтому введение в рамках этой концепции производной Галилея, как какого-то нового математического объекта со своим самостоятельным физическим смыслом, излишне. В рамках релятивистских же представлений рассмотрение производной Галилея бессодержательно по причине бессодержательности самих преобразований Галилея (в отличие от преобразований Лоренца). Но гиперконтинуальные представления о пространстве и времени делают использование галилеевой производной весьма востребованным, а равенство (17.7) - ложным.

Данный взгляд на пространство, время и электромагнитное поле в совокупности с применением производной Галилея приводит к новой, транскоординатной формулировке электродинамики [51]. Она обобщает общепринятую формулировку Герца-Хевисайда, что будет рассмотрено ниже.

Электромагнитное поле в изотропной однородной среде без дисперсии описывается в лабораторной и субстанциональной системах отсчета своими переменными (напряженность электрического поля $\mathbf{E}=\left(E^{\alpha}\right)$, $\mathbf{E}^{\prime}=\left(E^{\prime \alpha}\right)$ и магнитная индукция $\left.\mathbf{B}=\left(B^{\alpha}\right), \quad \mathbf{B}^{\prime}=\left(B^{\prime \alpha}\right)\right)$, постоянными (электрическая $\varepsilon_{0}$ и магнитная $\mu_{0}$, а также выражающаяся через них скорость света в вакууме $c=1 / \sqrt{\varepsilon_{0} \mu_{0}}$ ), параметрами (диэлектрическая и магнитная проницаемость $\varepsilon$ и $\mu$, а также плотность стороннего электрического заряда $\rho, \rho^{\prime}$, плотность электрического тока проводимости $\mathbf{j}=\left(j^{\alpha}\right)$, $\mathbf{j}^{\prime}=\left(j^{\prime \alpha}\right)$, электрический заряд $Q, Q^{\prime}$, электрический ток $\left.I, I^{\prime}\right)$, полевыми 


$$
\begin{array}{ccc}
\text { функциями } & \mathbf{E}=\mathbf{E}(\mathbf{r}, t)=\left(E^{\alpha}(\mathbf{r}, t)\right), \quad \mathbf{B}=\mathbf{B}(\mathbf{r}, t)=\left(B^{\alpha}(\mathbf{r}, t)\right), \\
\mathbf{E}^{\prime}=\mathbf{E}^{\prime}\left(v, \mathbf{r}^{\prime}, t\right)=\left(E^{\prime \alpha}\left(v, \mathbf{r}^{\prime}, t\right)\right), \mathbf{B}^{\prime}=\mathbf{B}^{\prime}\left(v, \mathbf{r}^{\prime}, t\right)=\left(B^{\prime \alpha}\left(v, \mathbf{r}^{\prime}, t\right)\right), \text { причем } \\
\mathbf{E}^{\prime}\left(0, \mathbf{r}^{\prime}, t\right)=\mathbf{E}\left(\mathbf{r}^{\prime}, t\right) ; \mathbf{B}^{\prime}\left(0, \mathbf{r}^{\prime}, t\right)=\mathbf{B}\left(v, \mathbf{r}^{\prime}, t\right) .
\end{array}
$$

В классической нерелятивистской электродинамике полагается:

$$
\mathbf{E}\left(\mathbf{r}^{\prime}+t v \mathbf{e}_{1}, t\right)=\mathbf{E}^{\prime}\left(v, \mathbf{r}^{\prime}, t\right) ; \mathbf{B}\left(\mathbf{r}^{\prime}+t v \mathbf{e}_{1}, t\right)=\mathbf{B}^{\prime}\left(v, \mathbf{r}^{\prime}, t\right),
$$

что есть применение общей формулы (17.3) инвариантности поля относительно скорости движения наблюдателя для случая электромагнитного поля. Предлагаемые нами гиперконтинуальные представления о пространстве и времени [52] выходят за рамки этой концепции, но объясняют природу этой неинвариантности не геометрией единого пространства-времени подобно теории относительности, а фундаментальными свойствами поля.

Интегральная форма уравнений Максвелла в представлении ГерцаХевисайда при указанных выше условиях (изотропия, однородность среды, отсутствие в ней дисперсии) являет собой следующую систему четырех интегральных уравнений электродинамики:

$$
\begin{array}{cc}
\int_{s} \mathbf{E} \cdot d s=\mathrm{Q} /\left(\varepsilon \varepsilon_{0}\right) ; & \int_{s} \mathbf{B} \cdot d s=0 ; \\
\int_{l} \mathbf{E} \cdot d l=-\frac{d}{d t} \int_{s} \mathbf{B} \cdot d s ; & \frac{c^{2}}{\varepsilon \mu} \int_{l} \mathbf{B} \cdot d l=\frac{\mathrm{I}}{\varepsilon \varepsilon_{0}}+\frac{d}{d t} \int_{s} \mathbf{E} \cdot d s,
\end{array}
$$

где $s, l$ - произвольная двумерная замкнутая (для первых двух уравнений) или открытая (для вторых двух) поверхность и ограничивающий ее замкнутый контур, не обязательно совпадающий с электрическим контуром.

Если на среду наложить еще дополнительное условие отсутствия свободных зарядов и токов, то последние два уравнения (17.10) примут вид:

$$
\int_{l} E \cdot d l=-\frac{d}{d t} \int_{s} B \cdot d s, \underset{l}{f} B \cdot d l=\frac{\varepsilon \mu}{c^{2}} \frac{d}{d t} \int_{s} E \cdot d s .
$$

Они представляют собой интегральную форму закона индукции Фарадея и теоремы о циркуляции магнитного поля в лабораторной системе отсчета для данного частного случая среды.

Эти два закона имеют взаимно симметричный вид с точностью до скалярного множителя, в силу чего их анализ идентичен. Рассмотрим более подробно, например, первый закон. В опытах Фарадея экспериментально установлено, что в контуре возникают одинаковые токи независимо от того, движется ли этот контур относительно токонесущего контура или покоится, а движется токонесущий контур, лишь бы их относительное движение в обоих случаях было одинаковым (галилеева инвариантность закона Фарадея). Поэтому поток через контур может изменяться вследствие изменения магнитного поля во времени, а также из-за того, что при перемещении контура изменяется положение его границы [56]. Соответствующее обобщение законов (17.11) на случай контура, движущегося в лабораторной и покоящегося в субстанциональной системе отсчета, имеет вид: 


$$
\int_{l} E^{\prime} \cdot d l=-\frac{d}{d t} \int_{s} B \cdot d s, \int_{l} B^{\prime} \cdot d l=\frac{\varepsilon \mu}{c^{2}} \frac{d}{d t} \int_{s} E \cdot d s,
$$

где $E^{\prime}$ и $B^{\prime}$ описывают поля в элементе $d l$ в субстанциональной системе отсчета, то есть в такой инерциальной системе отсчета, в которой $d l$ покоится; именно такое электрическое поле вызывает появление тока в случае наличия реального электрического контура в этом месте. Уравнения (17.12) весьма интересны и необычны с математической точки зрения, так как они связывают между собой полевые переменные в разных инерциальных системах отсчета (назовем такие уравнения транскоординатными). Именно использование транскоординатных уравнений позволяет адекватно описывать физические поля в гиперконтинууме. При этом в данном случае речь идет не просто о транскоординатности уравнений (17.12), а об их глобальной транскоординатности, обеспечиваемой использованием галилеевой производной (связываемые ими инерциальные системы отсчета могут двигаться друг относительно друга с произвольной скоростью, а не обязательно бесконечно малой).

Возвращаясь к системе уравнений (17.10), можно констатировать, что область ее применимости ограничена требованием состояния покоя контуpa $l$ в лабораторной системе отсчета. Если снять это ограничение, потребовав лишь состояния покоя контура $l$ в субстанциональной системе отсчета, то получится известное представление уравнений Максвелла (мы называем его транскоординатным [51]), интегральная форма которых будет являть собой в нем систему обобщающих (17.10) четырех интегральных уравнений электродинамики движущихся сред:

$$
\begin{array}{cc}
\int_{s} \mathbf{E} \cdot d s=\mathrm{Q} /\left(\varepsilon \varepsilon_{0}\right) ; & \int_{s} \mathbf{B} \cdot d s=0 ; \\
\int_{l} \mathbf{E}^{\prime} \cdot d l=-\frac{d}{d t} \int_{s} \mathbf{B} \cdot d s ; & \frac{c^{2}}{\varepsilon \mu} \int_{l} \mathbf{B}^{\prime} \cdot d l=\frac{\mathrm{I}^{\prime}}{\varepsilon \varepsilon_{0}}+\frac{d}{d t} \int_{s} \mathbf{E} \cdot d s .
\end{array}
$$

Если транскоординатное представление уравнений Максвелла (как в рассмотренной интегральной, так и в рассматриваемой ниже дифференциальной формах) интерпретировать в контексте описания электромагнитного поля в пространственно-временном гиперконтинууме, то нужно считать, что равенства (17.8) всегда выполняются, а (17.9) - в общем случае нет.

Уравнения (17.12) и (17.13) известны в классической электродинамике $[56,57]$. Возникает вопрос, как перейти от уравнений в интегральной форме (17.12) и (17.13) к соответствующим уравнениям в дифференциальной форме наиболее адекватным физической реальности образом.

Дифференциальная форма уравнений Максвелла в представлении ГерцаХевисайда являет собой следующую систему четырех соответствующих интегральным уравнениям (17.10) дифференциальных уравнений электродинамики, относящихся к лабораторной системе отсчета:

$$
\begin{array}{cc}
\nabla \cdot \mathbf{E}=\rho /\left(\varepsilon \varepsilon_{0}\right) ; & \nabla \cdot \mathbf{B}=0 ; \\
\nabla \times \mathbf{E}=-\partial \mathbf{B} / \partial t ; & \nabla \times \mathbf{B}=\mu \mu_{0} \mathbf{j}+\left(\varepsilon \mu / c^{2}\right)(\partial \mathbf{E} / \partial t) .
\end{array}
$$


Уравнения (17.14) традиционно успешно используются в электродинамике, но, как будет показано ниже, при этом имеют существенный недостаток - область их применимости ограничена случаем совпадения лабораторной и субстанциональной систем отсчета $(v=0)$, то есть эти уравнения лишены математических средств адекватного описания перехода от одной инерциальной системы отсчета к другой, всецело привязывая процесс к одной (лабораторной) системе отсчета.

В [56] на примере закона Фарадея сформулирован следующий подход к переходу от интегральной к дифференциальной форме уравнений электродинамики: «Закон Фарадея можно записать также и в дифференциальной форме, если воспользоваться теоремой Стокса и считать контур покоящимся в выбранной системе отсчета (для того, чтобы $E$ и $B$ были определены в одной и той же системе отсчета)». Этот подход отвечает концепции инвариантности физического поля относительно скорости движения наблюдателя, предполагая простой отказ от транскоординатности уравнений посредством применения (17.9). Но если мы отвергаем данную концепцию, то должны отвергнуть и данный подход. Тем самым, дифференциальная форма соответствующих уравнений должна быть такой же транскоординатной, как и интегральная (17.12) и (17.13).

В соответствии с данным традиционным подходом, в [57] вводится операция дифференцирования по времени в движущейся (субстанциональной) системе отсчета, обозначаемая там через $\partial^{\prime} / \partial t$. При этом негласно полагается, что в точке пространства, которая в субстанциональной системе отсчета имеет радиус-вектор $\mathbf{r}^{\prime}$, измерение полевой переменной в лабораторной системе отсчета равносильно ее измерению в той же самой субстанциональной системе отсчета. Но вне концепции инвариантности физического поля относительно скорости движения наблюдателя эти измерения не равносильны. Поэтому измерение нужно ограничивать лабораторной системой отсчета, не перенося его результаты на субстанциональную. Так мы приходим к производной Галилея (17.5), оставляющей уравнения электродинамики в дифференциальной форме глобально транскоординатными.

Искомые глобально транскоординатные дифференциальные уравнения электродинамики, соответствующие интегральным уравнениям (17.12) и использующие галилееву производную, имеют вид:

$$
\nabla \times E^{\prime}=-\frac{\partial^{\prime} B}{\partial t} ; \nabla \times B^{\prime}=\frac{\varepsilon \mu}{c^{2}} \frac{\partial^{\prime} E}{\partial t} .
$$

Они являются обобщением на случай несовпадения лабораторной и субстанциональной систем отсчета $(\mathbf{v} \neq 0)$ известных дифференциальных уравнений Максвелла:

$$
\nabla \times E=-\frac{\partial B}{\partial t} ; \nabla \times B=\frac{\varepsilon \mu}{c^{2}} \frac{\partial E}{\partial t} .
$$

Дифференциальная форма уравнений Максвелла в транскоординатном представлении для случая изотропной, однородной среды без дисперсии 
являет собой следующую систему четырех новых глобально транскоординатных дифференциальных уравнений электродинамики:

$$
\begin{gathered}
\nabla \cdot \mathbf{E}(\mathbf{r}, t)=\frac{\rho(\mathbf{r}, t)}{\varepsilon \varepsilon_{0}} ; \nabla \cdot \mathbf{B}(\mathbf{r}, t)=0 ; \\
\nabla \times \mathbf{E}^{\prime}\left(v, \mathbf{r}^{\prime}, t\right)=-\frac{\partial^{\prime} \mathbf{B}}{\partial t}\left(v, \mathbf{r}^{\prime}, t\right) ; \\
\nabla \times \mathbf{B}^{\prime}\left(v, \mathbf{r}^{\prime}, t\right)=\mu \mu_{0} \mathbf{j}^{\prime}\left(v, \mathbf{r}^{\prime}, t\right)+\frac{\varepsilon \mu}{c^{2}} \frac{\partial^{\prime} \mathbf{E}}{\partial t}\left(v, \mathbf{r}^{\prime}, t\right),
\end{gathered}
$$

где $\partial^{\prime} \mathbf{E} / \partial t, \partial^{\prime} \mathbf{B} / \partial t$ - производные Галилея полевых функций, выражаемые через частные производные по времени и конвективные производные тех же полевых функций в лабораторной системе отсчета равенствами:

$$
\begin{aligned}
& \frac{\partial^{\prime} \mathbf{E}}{\partial t}\left(v, \mathbf{r}^{\prime}, t\right)=\frac{\partial \mathbf{E}\left(\mathbf{r}^{\prime}+t v \mathbf{e}_{1}, t\right)}{\partial t}+\left(v \mathbf{e}_{1} \cdot \nabla\right) \mathbf{E}\left(\mathbf{r}^{\prime}+t v \mathbf{e}_{1}, t\right) \\
& \frac{\partial^{\prime} \mathbf{B}}{\partial t}\left(v, \mathbf{r}^{\prime}, t\right)=\frac{\partial \mathbf{B}\left(\mathbf{r}^{\prime}+t v \mathbf{e}_{1}, t\right)}{\partial t}+\left(v \mathbf{e}_{1} \cdot \nabla\right) \mathbf{B}\left(\mathbf{r}^{\prime}+t v \mathbf{e}_{1}, t\right) .
\end{aligned}
$$

При $v=0$ (17.17), (17.18) переходит в (17.14).

В частном случае отсутствия свободных зарядов и токов уравнения (17.17), (17.18) примут вид:

$$
\begin{gathered}
\nabla \cdot \mathbf{E}(\mathbf{r}, t)=0 ; \nabla \cdot \mathbf{B}(\mathbf{r}, t)=0 ; \\
\nabla \times \mathbf{E}^{\prime}\left(v, \mathbf{r}^{\prime}, t\right)=-\frac{\partial^{\prime} \mathbf{B}}{\partial t}\left(v, \mathbf{r}^{\prime}, t\right) ; \nabla \times \mathbf{B}^{\prime}\left(v, \mathbf{r}^{\prime}, t\right)=\frac{\varepsilon \mu}{c^{2}} \frac{\partial^{\prime} \mathbf{E}}{\partial t}\left(v, \mathbf{r}^{\prime}, t\right) .
\end{gathered}
$$

При $v=0$ (17.21), (17.22) переходит в следующую общеизвестную систему уравнений Максвелла:

$$
\begin{array}{cc}
\nabla \cdot \mathbf{E}(\mathbf{r}, t)=0 ; & \nabla \cdot \mathbf{B}(\mathbf{r}, t)=0 \\
\nabla \times \mathbf{E}(\mathbf{r}, t)=-\frac{\partial \mathbf{B}(\mathbf{r}, t)}{\partial t} ; & \nabla \times \mathbf{B}(\mathbf{r}, t)=\frac{\varepsilon \mu}{c^{2}} \frac{\partial \mathbf{E}(\mathbf{r}, t)}{\partial t} .
\end{array}
$$

Векторным произведением оператора набла на обе части уравнений (17.16) с их взаимной подстановкой друг в друга получают известные волновые дифференциальные уравнения

$$
c^{2} \nabla^{2} E=\varepsilon \mu \frac{\partial^{2} E}{\partial t^{2}} ; c^{2} \nabla^{2} B=\varepsilon \mu \frac{\partial^{2} B}{\partial t^{2}} .
$$

Их недостаток - отсутствие транскоординатности, они справедливы только в случае совпадения лабораторной и субстанциональной систем отсчета $(\mathbf{v}=0)$. Аналогично, векторным произведением набла на обе части уравнений (17.15) с их взаимной подстановкой друг в друга, получим новые уравнения электродинамики - глобально транскоординатные волновые дифференциальные уравнения, использующие галилееву производную полевых функций и обобщающие уравнения (17.24) на случай $\mathbf{v} \neq 0$ :

$$
c^{2} \nabla^{2} E^{\prime}=\varepsilon \mu \frac{\partial^{\prime 2} E}{\partial t^{2}} ; c^{2} \nabla^{2} B^{\prime}=\varepsilon \mu \frac{\partial^{\prime 2} B}{\partial t^{2}} .
$$

Исследуем подробнее уравнение вида (17.25) применительно к произвольным полевым функциям $\Phi(x, t)$ и $\Phi^{\prime}\left(v, x^{\prime}, t\right)$ для случая плоской волны 
с волновым вектором, коллинеарным $\mathbf{v}=(v, 0,0)$ и осям $O X, O^{\prime} X^{\prime}$, координаты по которым задаются переменными $x, x^{\prime}$. В этом случае уравнение оказывается одномерным, а полевые функции - скалярными:

$$
c^{2} \frac{\partial^{2}}{\partial x^{\prime 2}} \Phi^{\prime}\left(v, x^{\prime}, t\right)=\varepsilon \mu \frac{\partial^{\prime 2} \Phi}{\partial t^{2}}\left(v, x^{\prime}, t\right)=\varepsilon \mu \frac{d^{2}}{d t^{2}} \Phi\left(x^{\prime}+v t, t\right) .
$$

Если взять производную в правой части (17.26), уравнение примет вид:

$$
\begin{aligned}
& \frac{c^{2}}{\varepsilon \mu} \frac{\partial^{2}}{\partial x^{\prime 2}} \Phi^{\prime}\left(v, x^{\prime}, t\right)=\left(\frac{\partial^{2}}{\partial t^{2}}+2 v \frac{\partial^{2}}{\partial t \partial x}+v^{2} \frac{\partial^{2}}{\partial x^{2}}\right) \Phi\left(x^{\prime}+v t, t\right)= \\
& =\left(\frac{\partial}{\partial t}+v \frac{\partial}{\partial x}\right)^{2} \Phi\left(x^{\prime}+v t, t\right) .
\end{aligned}
$$

При $v=0$ (17.26) и (17.27) вырождается в одномерный вариант волнового уравнения вида (17.24):

$$
c^{2} \frac{\partial^{2}}{\partial x^{2}} \Phi(x, t)=\varepsilon \mu \frac{\partial^{2}}{\partial t^{2}} \Phi(x, t) .
$$

Любое решение (17.28) определяется надлежащей суперпозицией монохроматических волн

$$
\Phi(x, t)=A \cos \left(\omega t-k_{x} x+\varphi\right)
$$

с подходящими значениями параметров $A \geq 0, \omega>0, k_{x} \neq 0, \varphi \in \mathbb{R}$ - амплитуда, круговая частота, проекция волнового вектора на ось $O X$ и начальная фаза волны. При этом все волны (17.29) должны иметь одну и ту же фазовую скорость $\omega / k=c / \sqrt{\varepsilon \mu}$, где $k=\left|k_{x}\right|-$ волновое число. Будем искать функцию $\Phi^{\prime}\left(v, x^{\prime}, t\right)$, удовлетворяющую (17.26)-(17.29), тоже в виде монохроматической волны, но с зависящими от $v$ параметрами $A^{\prime}(v)$, $\omega^{\prime}(v), k_{x}^{\prime}(v), \varphi^{\prime}(v)$ :

$$
\Phi^{\prime}\left(v, x^{\prime}, t\right)=A^{\prime}(v) \cos \left(\omega^{\prime}(v) t-k_{x}^{\prime}(v) x^{\prime}+\varphi^{\prime}(v)\right),
$$

$\Phi^{\prime}\left(0, x^{\prime}, t\right)=\Phi\left(x^{\prime}, t\right), A^{\prime}(0)=A, \omega^{\prime}(0)=\omega, k_{x}^{\prime}(0)=k_{x}, \varphi^{\prime}(0)=\varphi$.

Подставим (17.29)-(17.30) в (17.27):

$$
\begin{aligned}
& c^{2} k_{x}^{\prime 2}(v) A^{\prime}(v) \cos \left(\omega^{\prime}(v) t-k_{x}^{\prime}(v) x^{\prime}+\varphi^{\prime}(v)\right)= \\
& =\varepsilon \mu\left(\omega-k_{x} v\right)^{2} A \cos \left(\omega t-k_{x}\left(x^{\prime}+v t\right)+\varphi\right) .
\end{aligned}
$$

Приравнивая одноименные параметры волны в левой части (17.31) и волны в правой, имеем:

$$
\begin{gathered}
A^{\prime}(v)=\left(\operatorname{sgn} k_{x}-\frac{\sqrt{\varepsilon \mu}}{c} v\right)^{2} A ; \omega^{\prime}(v)=\left|\omega-k_{x} v\right|=\left|1-\frac{\sqrt{\varepsilon \mu}}{c} v \operatorname{sgn} k_{x}\right| \omega ; \\
k_{x}^{\prime}(v)=k_{x} \operatorname{sgn}\left(\omega-k_{x} v\right) ; \quad k^{\prime}(v)=\left|k_{x}^{\prime}(v)\right|=k ; \\
\varphi^{\prime}(v)=\varphi \operatorname{sgn}\left(\omega-k_{x} v\right) ; \quad\left|\varphi^{\prime}(v)\right|=|\varphi| .
\end{gathered}
$$

Таким образом, при переходе от лабораторной к субстанциональной системе отсчета изменяются амплитуда и частота (17.32) монохроматической 
волны, а ее волновое число и модуль начальной фазы (17.33) остаются неизменными. При этом частота изменяется таким образом, что фазовая скорость волны в субстанциональной системе отсчета получается по классическому правилу сложения скоростей из ее фазовой скорости в лабораторной системе отсчета и скорости субстанциональной системы отсчета относительно лабораторной:

$$
\begin{gathered}
\omega^{\prime}(v) / k_{x}^{\prime}(v)=\omega^{\prime}(v) / k_{x}=\omega / k_{x}-v ; \\
\omega^{\prime}(v) / k^{\prime}(v)=\left|\omega / k-v \operatorname{sgn} k_{x}\right|=\left|c / \sqrt{\varepsilon \mu}-v \operatorname{sgn} k_{x}\right| .
\end{gathered}
$$

Из (17.32-17.34) видно, что если вектор фазовой скорости волны в лабораторной системе отсчета совпадает с вектором скорости субстанциональной системы отсчета в ней $\left(k_{x}>0, v=\omega / k\right)$, то в субстанциональной системе отсчета волна вообще исчезает $\left(A^{\prime}(v)=0\right)$. Таким образом, в отличие от теории относительности, в теории гиперконтинуума такую волну всегда можно уничтожить подходящим выбором системы отсчета. Если же относительно лабораторной системы отсчета субстанциальная система отсчета перегоняет волну, то при переходе от лабораторной системы отсчета к субстанциональной направление распространения волны меняется на противоположное. Если в лабораторной системе отсчета волна распространяется в положительном направлении, то при переходе в субстанциональную она будет удовлетворять волновому уравнению (17.35), а если в отрицательном, то уравнению (17.36):

$$
\begin{aligned}
& (c / \sqrt{\varepsilon \mu}-v)^{2} \partial^{2} \Phi^{\prime}\left(v, x^{\prime}, t\right) / \partial x^{\prime 2}=\partial^{2} \Phi^{\prime}\left(v, x^{\prime}, t\right) / \partial t^{2} ; \\
& (c / \sqrt{\varepsilon \mu}+v)^{2} \partial^{2} \Phi^{\prime}\left(v, x^{\prime}, t\right) / \partial x^{\prime 2}=\partial^{2} \Phi^{\prime}\left(v, x^{\prime}, t\right) / \partial t^{2} .
\end{aligned}
$$

Выбор инерциальной системы отсчета на роль лабораторной, вообще говоря, условен. Так, субстанциальную систему отсчета можно в свою очередь принять за лабораторную, а в роли субстанциальной рассматривать некую третью (дважды штрихованную) инерциальную систему отсчета с направленной в ту же сторону, что и $O X, O^{\prime} X^{\prime}$, пространственной осью координат $O^{\prime \prime} X^{\prime \prime}$, координата по которой задается переменной $x^{\prime \prime}$. Пусть, например, точка $O^{\prime \prime}$ движется в положительном направлении оси $O^{\prime} X^{\prime}$ со скоростью $\Delta v$. Волна в новых лабораторной и субстанциональной системах отсчета будет иметь одинаковое волновое число и модуль начальной фазы и будет описываться полевыми функциями $\Phi^{\prime}\left(v, x^{\prime}, t\right)$ и $\Phi^{\prime}\left(v+\Delta v, x^{\prime \prime}, t\right)$ соответственно. Роль уравнения (17.28) будет играть (17.35) или (17.36), роль функции волны (17.29) - функция (17.30), а роль уравнений (17.35), (17.36) - следующие волновые уравнения:

$$
\begin{aligned}
& (c / \sqrt{\varepsilon \mu}-(v+\Delta v))^{2} \partial^{2} \Phi^{\prime}\left(v+\Delta v, x^{\prime \prime}, t\right) / \partial x^{\prime \prime 2}=\partial^{2} \Phi^{\prime}\left(v+\Delta v, x^{\prime \prime}, t\right) / \partial t^{2} ; \\
& (c / \sqrt{\varepsilon \mu}+(v+\Delta v))^{2} \partial^{2} \Phi^{\prime}\left(v+\Delta v, x^{\prime \prime}, t\right) / \partial x^{\prime \prime 2}=\partial^{2} \Phi^{\prime}\left(v+\Delta v, x^{\prime \prime}, t\right) / \partial t^{2} .
\end{aligned}
$$

Для (17.37) роль равенств (17.32), (17.33) будут играть следующие преобразования параметров волны: 


$$
\begin{gathered}
A^{\prime \prime}(v+\Delta v)=\left(\operatorname{sgn} k_{x}^{\prime}(v)-\frac{\sqrt{\varepsilon \mu} \cdot \Delta v}{c-\sqrt{\varepsilon \mu} \cdot v}\right)^{2} A^{\prime}(v) ; \\
\omega^{\prime \prime}(v+\Delta v)=\left|\omega^{\prime}(v)-k_{x}^{\prime}(v)\right| \Delta v ; \\
k_{x}^{\prime \prime}(v+\Delta v)=k_{x}^{\prime}(v) \operatorname{sgn}\left(\omega^{\prime}(v)-k_{x}^{\prime}(v) \Delta v\right) ; \\
\varphi^{\prime \prime}(v+\Delta v)=\varphi^{\prime}(v) \operatorname{sgn}\left(\omega^{\prime}(v)-k_{x}^{\prime}(v) \Delta v\right) .
\end{gathered}
$$

Для (17.38) соответствующие (17.39), (17.40) преобразования параметров определяются аналогично.

Последовательный переход от не штрихованной к штрихованной и далее к дважды штрихованной системе отсчета равносилен непосредственному переходу от не штрихованной к дважды штрихованной. Например, при $\operatorname{sgn} k_{x}^{\prime}(v)=\operatorname{sgn} k_{x}=1$ из (17.32), (17.39) можно получить

$$
A^{\prime \prime}(v+\Delta v)=(1-\sqrt{\varepsilon \mu}(v+\Delta v) / c)^{2} A,
$$

что получается и при непосредственном переходе к дважды штрихованной системе отсчета, так как (17.41) получается из (17.32) заменой $v$ на $v+\Delta v$. В данном случае роль уравнения (17.27) будет играть уравнение

$$
\begin{aligned}
& \left(\frac{c}{\sqrt{\varepsilon \mu}}-v\right)^{2} \frac{\partial^{2} \Phi^{\prime}\left(v+\Delta v, x^{\prime \prime}, t\right)}{\partial x^{\prime \prime}}=\frac{\partial^{2} \Phi^{\prime}\left(v, x^{\prime \prime}+\Delta v t, t\right)}{\partial t^{2}}+ \\
& \left(2 \Delta v \frac{\partial^{2}}{\partial t \partial x^{\prime}}+\Delta v^{2} \frac{\partial^{2}}{\partial x^{\prime 2}}\right) \Phi^{\prime}\left(v, x^{\prime \prime}+\Delta v t, t\right) .
\end{aligned}
$$

Для производных произвольного п-го порядка $\partial^{n} \Phi^{\prime}\left(v+\Delta v, x^{\prime \prime}, t\right) / \partial x^{\prime \prime n}$ и $\partial^{n} \Phi^{\prime}\left(v, x^{\prime}, t\right) / \partial x^{\prime n} \quad$ можно использовать единое обозначение $\partial^{n} \Phi^{\prime}(v+\Delta v, x, t) / \partial x^{n}$ и $\partial^{n} \Phi^{\prime}(v, x, t) / \partial x^{n}$ соответственно $(n=\overline{1, \infty})$, означающее просто производную по второму аргументу. В соответствии с этим, после подстановки (17.35) в (17.42) получим:

$$
\begin{aligned}
& \left(\frac{c}{\sqrt{\varepsilon \mu}}-v\right)^{2} \frac{\partial^{2}}{\partial x^{2}}\left(\frac{\Phi^{\prime}(v+\Delta v, x, t)-\Phi^{\prime}(v, x+\Delta v t, t)}{\Delta v}\right)= \\
& =\left(2 \frac{\partial^{2}}{\partial t \partial x}+\Delta v \frac{\partial^{2}}{\partial x^{2}}\right) \Phi^{\prime}(v, x+\Delta v t, t) .
\end{aligned}
$$

Пусть $\Delta v \rightarrow 0$. Введем еще одну новую производную - транскоординатную. В одномерной системе пространственных координат она имеет вид:

$$
\frac{\partial^{\prime} \Phi^{\prime}(v, x, t)}{\partial^{\prime} v}=\lim _{\Delta v \rightarrow 0} \frac{\Phi^{\prime}(v+\Delta v, x, t)-\Phi^{\prime}(v, x+\Delta v t, t)}{\Delta v} .
$$

В (17.44) величины $\Phi^{\prime}(v, x+\Delta v t, t)$ и $\Phi^{\prime}(v+\Delta v, x, t)$ описывают физическое поле в одной и той же точке пространства, но в разных системах отсчета (штрихованной и движущейся относительно нее со скоростью $\Delta v$ дважды штрихованной соответственно). В рамках концепции инвариантности поля относительно скорости движения наблюдателя они равны: 


$$
\Phi^{\prime}(v, x+\Delta v t, t)=\Phi^{\prime}(v+\Delta v, x, t) .
$$

Физический смысл (17.3) и (17.45) одинаков применительно к разным парам систем отсчета. Однако вне рамок указанной концепции при переходе от штрихованной к дважды штрихованной системе отсчета полевая функция в данной точке пространства испытывает приращение, предел отношения которого к $\Delta v$ при $\Delta v \rightarrow 0$ дает транскоординатную производную (17.44). Ее можно обобщить на случай высших порядков $(n=\overline{1, \infty})$ :

$$
\begin{gathered}
\frac{\partial^{\prime 1} \Phi^{\prime}(v, x, t)}{\partial^{\prime} v^{1}}=\frac{\partial^{\prime} \Phi^{\prime}(v, x, t)}{\partial^{\prime} v} ; \\
\frac{\partial^{\prime n+1} \Phi^{\prime}(v, x, t)}{\partial^{\prime} v^{n+1}}=\lim _{\Delta v \rightarrow 0} \frac{\frac{\partial^{\prime n} \Phi^{\prime}(v+\Delta v, x, t)}{\partial^{\prime} v^{n}}-\frac{\partial^{\prime n} \Phi^{\prime}(v, x+\Delta v t, t)}{\partial^{\prime} v^{n}}}{\Delta v} .
\end{gathered}
$$

Используя транскоординатные производные первых двух порядков (17.46), можно представить приращение полевой функции в виде соответствующей частичной суммы ряда Тейлора:

$$
\Phi^{\prime}(v+\Delta v, x, t)-\Phi^{\prime}(v, x+\Delta v t, t) \approx \frac{\partial^{\prime} \Phi^{\prime}(v, x, t)}{\partial^{\prime} v} \Delta v+\frac{1}{2} \frac{\partial^{\prime 2} \Phi^{\prime}(v, x, t)}{\partial^{\prime} v^{2}} \Delta v^{2} .
$$

Подставляя (17.47) в (17.43), приравнивая между собой члены с одинаковыми степенями $\Delta v$ в левой и правой частях получившегося равенства, устремляя $\Delta v \rightarrow 0$, учитывая то, что при этом $\Phi^{\prime}(v, x+\Delta v t, t) \rightarrow \Phi^{\prime}(v, x, t)$ и добавляя равенство (17.35) в новой форме записи (с использованием переменной $x$ вместо $x^{\prime}$, получим следующую систему трех уравнений:

$$
\left\{\begin{array}{l}
\left(\frac{c}{\sqrt{\varepsilon \mu}}-v\right)^{2} \frac{\partial^{2} \Phi^{\prime}(v, x, t)}{\partial x^{2}}=\frac{\partial^{2} \Phi^{\prime}\left(v, x^{\prime}, t\right)}{\partial t^{2}}, \\
\left(\frac{c}{\sqrt{\varepsilon \mu}}-v\right)^{2} \frac{\partial \partial^{\prime} \Phi^{\prime}(v, x, t)}{\partial x \partial^{\prime} v}=2 \frac{\partial \Phi^{\prime}\left(v, x^{\prime}, t\right)}{\partial t}, \\
\left(\frac{c}{\sqrt{\varepsilon \mu}}-v\right)^{2} \frac{\partial^{\prime 2} \Phi^{\prime}(v, x, t)}{\partial^{\prime} v^{2}}=2 \Phi^{\prime}\left(v, x^{\prime}, t\right)
\end{array}\right.
$$

Систему (17.48) можно записать в проиндексированной по $\alpha$ форме

$$
\left(\left(\frac{c}{\sqrt{\varepsilon \mu}}-v\right)^{2} \frac{\partial^{2-\alpha} \partial^{\prime \alpha}}{\partial x^{2-\alpha} \partial^{\prime} v^{\alpha}}-2^{\operatorname{sgn} \alpha} \frac{\partial^{2-\alpha}}{\partial t^{2-\alpha}}\right) \Phi^{\prime}\left(v, x^{\prime}, t\right)=0, \alpha=\overline{0,2}
$$

или в операторной форме

$$
\nLeftarrow \Phi^{\prime}\left(v, x^{\prime}, t\right)=0 \text {, }
$$

где $\nLeftarrow=(\nLeftarrow \alpha) ; \propto \alpha \alpha^{\alpha}=\left(\left(\frac{c}{\sqrt{\varepsilon \mu}}-v\right)^{2} \frac{\partial^{2-\alpha} \partial^{\prime \alpha}}{\partial x^{2-\alpha} \partial^{\prime} v^{\alpha}}-2^{\operatorname{sgn} \alpha} \frac{\partial^{2-\alpha}}{\partial t^{2-\alpha}}\right)-$ подходящий вариант одномерного (случай одной оси пространственных координат) дифференциального оператора Дубровина, обобщающего оператор Даламбера 
口, который оказывается одной из трех (нулевой) его компонент для лабораторной системы отсчета, то есть $\alpha=0, v=0$. Дифференциальное уравнение (17.49) или (17.50) есть гиперконтинуальное одномерное однородное волновое уравнение, обобщающее, подобно дифференциальному уравнению (17.26) или (17.27), известное одномерное однородное волновое уравнение (17.28). Принципиальное отличие между ними состоит в том, что (17.26), (17.27) является глобально транскоординатной формой гиперконтинуального волнового уравнения, а (17.49), (17.50) - его локально транскоординатной формой. Локальная транскоординатность означает, что уравнение связывает инерциальные системы отсчета, движущиеся друг относительно друга с бесконечно малой скоростью.

Транскоординатность гиперконтинуальных волновых уравнений обеспечивается использованием в них подходящих производных полевых функций. Использование производной Галилея сообщает уравнению глобальную транскоординатность, транскоординатной производной - локальную.

Таким образом, предлагается новый подход к развитию математического аппарата электродинамики в направлении более адекватного описания перехода от одной инерциальной системы отсчета к другой на основе гиперконтинуальных представлений о пространстве и времени за счет введения в рассмотрение глобально и локально транскоординатных уравнений, использующих новые галилееву и транскоординатную производные полевых функций, а также новый дифференциальный оператор Дубровина, обобщающий оператор Даламбера. Этот подход приводит к переформулированию электродинамики с переходом от традиционной формулировки ГерцаХевисайда к новой транскоординатной. При этом сразу возникает вопрос о том, какой вид имеют преобразования электромагнитного поля при переходе от одной инерциальной системы отсчета к другой, и будут ли эти преобразования преобразованиями Менде.

Ответу на данный вопрос посвящена статья [58]. Далее по материалам работы [58] в рамках транскоординатной формулировки электродинамики и гиперконтинуальных представлений о пространстве и времени выведем преобразования электромагнитного поля при переходе от одной инерциальной системы отсчета к другой.

Конвективные производные полевых функций в (17.19), (17.20) можно записать в виде:

$$
\begin{aligned}
& \left(v \mathbf{e}_{1} \cdot \nabla\right) \mathbf{E}\left(\mathbf{r}^{\prime}+t v \mathbf{e}_{1}, t\right)=v\left(\nabla \cdot \mathbf{E}\left(\mathbf{r}^{\prime}+t v \mathbf{e}_{1}, t\right)\right) \mathbf{e}_{1}-\nabla \times\left(v \mathbf{e}_{1} \times \mathbf{E}\left(\mathbf{r}^{\prime}+t v \mathbf{e}_{1}, t\right)\right) ; \\
& \left(v \mathbf{e}_{1} \cdot \nabla\right) \mathbf{B}\left(\mathbf{r}^{\prime}+t v \mathbf{e}_{1}, t\right)=v\left(\nabla \cdot \mathbf{B}\left(\mathbf{r}^{\prime}+t v \mathbf{e}_{1}, t\right)\right) \mathbf{e}_{1}-\nabla \times\left(v \mathbf{e}_{1} \times \mathbf{B}\left(\mathbf{r}^{\prime}+t v \mathbf{e}_{1}, t\right)\right) .
\end{aligned}
$$

В силу первых двух уравнений (17.22) с учетом (17.1)-(17.2) имеем:

$$
\nabla \cdot \mathbf{E}\left(\mathbf{r}^{\prime}+t v \mathbf{e}_{1}, t\right)=0 ; \nabla \cdot \mathbf{B}\left(\mathbf{r}^{\prime}+t v \mathbf{e}_{1}, t\right)=0
$$

Подставив (17.53) в (17.51), (17.52), получим равенства для конвективных производных:

$$
\begin{aligned}
& \left(v \mathbf{e}_{1} \cdot \nabla\right) \mathbf{E}\left(\mathbf{r}^{\prime}+t v \mathbf{e}_{1}, t\right)=-\nabla \times\left(v \mathbf{e}_{1} \times \mathbf{E}\left(\mathbf{r}^{\prime}+t v \mathbf{e}_{1}, t\right)\right) ; \\
& \left(v \mathbf{e}_{1} \cdot \nabla\right) \mathbf{B}\left(\mathbf{r}^{\prime}+t v \mathbf{e}_{1}, t\right)=-\nabla \times\left(v \mathbf{e}_{1} \times \mathbf{B}\left(\mathbf{r}^{\prime}+t v \mathbf{e}_{1}, t\right)\right) .
\end{aligned}
$$


После подстановки (17.54), (17.55) в (17.19), (17.20) имеем другой вид галилеевых производных:

$$
\begin{aligned}
& \frac{\partial^{\prime} \mathbf{E}}{\partial t}\left(v, \mathbf{r}^{\prime}, t\right)=\frac{\partial \mathbf{E}\left(\mathbf{r}^{\prime}+t v \mathbf{e}_{1}, t\right)}{\partial t}-\nabla \times\left(v \mathbf{e}_{1} \times \mathbf{E}\left(\mathbf{r}^{\prime}+t v \mathbf{e}_{1}, t\right)\right) ; \\
& \frac{\partial^{\prime} \mathbf{B}}{\partial t}\left(v, \mathbf{r}^{\prime}, t\right)=\frac{\partial \mathbf{B}\left(\mathbf{r}^{\prime}+t v \mathbf{e}_{1}, t\right)}{\partial t}-\nabla \times\left(v \mathbf{e}_{1} \times \mathbf{B}\left(\mathbf{r}^{\prime}+t v \mathbf{e}_{1}, t\right)\right) .
\end{aligned}
$$

Подстановка галилеевых производных (17.56), (17.57) в последние два равенства (17.22) дает:

$$
\begin{gathered}
\nabla \times \mathbf{E}^{\prime}\left(v, \mathbf{r}^{\prime}, t\right)=-\partial \mathbf{B}\left(\mathbf{r}^{\prime}+t v \mathbf{e}_{1}, t\right) / \partial t+\nabla \times\left(v \mathbf{e}_{1} \times \mathbf{B}\left(\mathbf{r}^{\prime}+t v \mathbf{e}_{1}, t\right)\right) ; \\
\nabla \times \mathbf{B}^{\prime}\left(v, \mathbf{r}^{\prime}, t\right)=\left(\varepsilon \mu / c^{2}\right)\left(\partial \mathbf{E}\left(\mathbf{r}^{\prime}+t v \mathbf{e}_{1}, t\right) / \partial t-\nabla \times\left(v \mathbf{e}_{1} \times \mathbf{E}\left(\mathbf{r}^{\prime}+t v \mathbf{e}_{1}, t\right)\right)\right) .
\end{gathered}
$$

Подставив последние два уравнения (17.23) в (17.58)-(17.59), получим:

$$
\begin{gathered}
\nabla \times \mathbf{E}^{\prime}\left(v, \mathbf{r}^{\prime}, t\right)=\nabla \times \mathbf{E}\left(\mathbf{r}^{\prime}+t v \mathbf{e}_{1}, t\right)+\nabla \times\left(v \mathbf{e}_{1} \times \mathbf{B}\left(\mathbf{r}^{\prime}+t v \mathbf{e}_{1}, t\right)\right) ; \\
\nabla \times \mathbf{B}^{\prime}\left(v, \mathbf{r}^{\prime}, t\right)=\nabla \times \mathbf{B}\left(\mathbf{r}^{\prime}+t v \mathbf{e}_{1}, t\right)-\left(\varepsilon \mu / c^{2}\right) \nabla \times\left(v \mathbf{e}_{1} \times \mathbf{E}\left(\mathbf{r}^{\prime}+t v \mathbf{e}_{1}, t\right)\right) .
\end{gathered}
$$

Опустим операцию ротора в обеих частях равенств (17.60), (17.61):

$$
\begin{gathered}
\mathbf{E}^{\prime}\left(v, \mathbf{r}^{\prime}, t\right)=\mathbf{E}\left(\mathbf{r}^{\prime}+t v \mathbf{e}_{1}, t\right)+v \mathbf{e}_{1} \times \mathbf{B}\left(\mathbf{r}^{\prime}+t v \mathbf{e}_{1}, t\right) ; \\
\mathbf{B}^{\prime}\left(v, \mathbf{r}^{\prime}, t\right)=\mathbf{B}\left(\mathbf{r}^{\prime}+t v \mathbf{e}_{1}, t\right)-\left(\varepsilon \mu / c^{2}\right)\left(v \mathbf{e}_{1} \times \mathbf{E}\left(\mathbf{r}^{\prime}+t v \mathbf{e}_{1}, t\right)\right) .
\end{gathered}
$$

Кроме штрихованной системы отсчета, движущейся относительно лабораторной со скоростью $v$, введем еще относительно подвижную систему отсчета - дважды штрихованную, движущуюся в том же направлении с другой скоростью $v+\Delta v$ относительно лабораторной. Тем самым, дважды штрихованная система отсчета движется относительно штрихованной со скоростью $\Delta v$, так что штрихованную систему отсчета можно принять за новую лабораторную (относительно неподвижную), а дважды штрихованную - за новую субстанциональную.

Равенства (17.62), (17.63) для них запишем с учетом замены радиусвектора $\mathbf{r}^{\prime}$ на $\mathbf{r}^{\prime \prime}$ :

$$
\begin{gathered}
\mathbf{E}^{\prime}\left(v+\Delta v, \mathbf{r}^{\prime \prime}, t\right)=\mathbf{E}^{\prime}\left(v, \mathbf{r}^{\prime \prime}+t \Delta v \mathbf{e}_{1}, t\right)+\Delta v \mathbf{e}_{1} \times \mathbf{B}^{\prime}\left(v, \mathbf{r}^{\prime \prime}+t \Delta v \mathbf{e}_{1}, t\right) \\
\mathbf{B}^{\prime}\left(v+\Delta v, \mathbf{r}^{\prime \prime}, t\right)=\mathbf{B}^{\prime}\left(v, \mathbf{r}^{\prime \prime}+t \Delta v \mathbf{e}_{1}, t\right)-\left(\varepsilon \mu / c^{2}\right) \Delta v \mathbf{e}_{1} \times \mathbf{E}^{\prime}\left(v, \mathbf{r}^{\prime \prime}+t \Delta v \mathbf{e}_{1}, t\right) .
\end{gathered}
$$

Запишем равенства (17.64), (17.65) в следующем виде:

$$
\begin{gathered}
\frac{\mathbf{E}^{\prime}\left(v+\Delta v, \mathbf{r}^{\prime \prime}, t\right)-\mathbf{E}^{\prime}\left(v, \mathbf{r}^{\prime \prime}+t \Delta v \mathbf{e}_{1}, t\right)}{\Delta v}=\mathbf{e}_{1} \times \mathbf{B}^{\prime}\left(v, \mathbf{r}^{\prime \prime}+t \Delta v \mathbf{e}_{1}, t\right) ; \\
\frac{\mathbf{B}^{\prime}\left(v+\Delta v, \mathbf{r}^{\prime \prime}, t\right)-\mathbf{B}^{\prime}\left(v, \mathbf{r}^{\prime \prime}+t \Delta v \mathbf{e}_{1}, t\right)}{\Delta v}=-\frac{\varepsilon \mu}{c^{2}} \mathbf{e}_{1} \times \mathbf{E}^{\prime}\left(v, \mathbf{r}^{\prime \prime}+t \Delta v \mathbf{e}_{1}, t\right) .
\end{gathered}
$$

В (17.66), (17.67) величины $\quad \mathbf{E}^{\prime}\left(v, \mathbf{r}^{\prime \prime}+t \Delta v \mathbf{e}_{1}, t\right), \quad \mathbf{B}^{\prime}\left(v, \mathbf{r}^{\prime \prime}+t \Delta v \mathbf{e}_{1}, t\right) \quad$ и $\mathbf{E}^{\prime}\left(v+\Delta v, \mathbf{r}^{\prime \prime}, t\right), \mathbf{B}^{\prime}\left(v+\Delta v, \mathbf{r}^{\prime \prime}, t\right)$ описывают электромагнитное поле в одной и той же точке пространства (среды), но в разных системах отсчета (штрихованной и дважды штрихованной). В рамках концепции инвариантности поля относительно скорости движения наблюдателя они равны: 


$$
\mathbf{E}^{\prime}\left(v, \mathbf{r}^{\prime \prime}+t \Delta v \mathbf{e}_{1}, t\right)=\mathbf{E}^{\prime}\left(v+\Delta v, \mathbf{r}^{\prime \prime}, t\right) ; \mathbf{B}^{\prime}\left(v, \mathbf{r}^{\prime \prime}+t \Delta v \mathbf{e}_{1}, t\right)=\mathbf{B}^{\prime}\left(v+\Delta v, \mathbf{r}^{\prime \prime}, t\right),
$$

причем равенства (17.9) и (17.68) имеют одинаковый физический смысл, но применительно к разным парам систем отсчета. Однако вне рамок указанной концепции при переходе от штрихованной к дважды штрихованной системе отсчета полевая функция в данной точке пространства испытывает приращение, предел отношения которого к $\Delta v$ при $\Delta v \rightarrow 0$ дает впервые введенную в [7] транскоординатную производную полевой функции:

$$
\begin{gathered}
\frac{\partial^{\prime} \mathbf{E}^{\prime}\left(v, \mathbf{r}^{\prime \prime}, t\right)}{\partial^{\prime} v}=\lim _{\Delta v \rightarrow 0} \frac{\mathbf{E}^{\prime}\left(v+\Delta v, \mathbf{r}^{\prime \prime}, t\right)-\mathbf{E}^{\prime}\left(v, \mathbf{r}^{\prime \prime}+t \Delta v \mathbf{e}_{1}, t\right)}{\Delta v} ; \\
\frac{\partial^{\prime} \mathbf{B}^{\prime}\left(v, \mathbf{r}^{\prime \prime}, t\right)}{\partial^{\prime} v}=\lim _{\Delta v \rightarrow 0} \frac{\mathbf{B}^{\prime}\left(v+\Delta v, \mathbf{r}^{\prime \prime}, t\right)-\mathbf{B}^{\prime}\left(v, \mathbf{r}^{\prime \prime}+t \Delta v \mathbf{e}_{1}, t\right)}{\Delta v} .
\end{gathered}
$$

Равенства $(17.66),(17.67)$ при $\Delta v \rightarrow 0$ с учетом $(17.69,17.70)$ после замены $\mathbf{r}^{\prime \prime}$ на $\mathbf{r}$ принимают вид:

$$
\frac{\partial^{\prime} \mathbf{E}^{\prime}\left(v, \mathbf{r}^{\prime}, t\right)}{\partial^{\prime} v}=\mathbf{e}_{1} \times \mathbf{B}^{\prime}\left(v, \mathbf{r}^{\prime}, t\right) ; \frac{\partial^{\prime} \mathbf{B}^{\prime}\left(v, \mathbf{r}^{\prime}, t\right)}{\partial^{\prime} v}=-\frac{\varepsilon \mu}{c^{2}} \mathbf{e}_{1} \times \mathbf{E}^{\prime}\left(v, \mathbf{r}^{\prime}, t\right) .
$$

Если уравнения (17.22) являются глобально транскоординатными дифференциальными уравнениями электродинамики для случая изотропной однородной среды без дисперсии в отсутствии свободных зарядов и токов, то уравнения (17.71) представляют собой локально транскоординатные дифференциальные уравнения электродинамики для того же самого случая. Локальность транскоординатности, обеспечиваемая использованием транскоординатной производной, означает, что связываемые дифференциальными уравнениями инерциальные системы отсчета (условно говоря, штрихованная и дважды штрихованная) движутся друг относительно друга с бесконечно малой скоростью $\Delta v$. Уравнения (17.71) образуют систему, решая которую, можно получить преобразования электромагнитного поля при переходе из одной инерциальной системы отсчета в другую.

Применим систему уравнений (17.71) для получения преобразований электромагнитного поля при переходе от лабораторной системы отсчета к субстанциональной.

Опуская аргументы, запишем векторные произведения в (17.71) в виде:

$$
\begin{aligned}
\mathbf{e}_{1} \times \mathbf{B}^{\prime} & =\mathbf{e}_{1} \times\left(B^{\prime 1} \mathbf{e}_{1}+B^{\prime 2} \mathbf{e}_{2}+B^{\prime 3} \mathbf{e}_{3}\right)=B^{\prime 2} \mathbf{e}_{3}-B^{\prime 3} \mathbf{e}_{2} ; \\
\mathbf{e}_{1} \times \mathbf{E}^{\prime}=\mathbf{e}_{1} \times\left(E^{\prime 1} \mathbf{e}_{1}+E^{\prime 2} \mathbf{e}_{2}+E^{\prime 3} \mathbf{e}_{3}\right) & =E^{\prime 2} \mathbf{e}_{3}-E^{\prime 3} \mathbf{e}_{2} .
\end{aligned}
$$

С учетом $(17.72,17.73)$ система уравнений (17.71) разбивается на две независимые системы из двух уравнений каждая и два независимых уравнения:

$$
\left\{\begin{array}{l}
\frac{\partial^{\prime} E^{\prime 2}}{\partial^{\prime} v}=-B^{\prime 3}, \\
\frac{\partial^{\prime} B^{\prime 3}}{\partial^{\prime} v}=-\frac{\varepsilon \mu}{c^{2}} E^{\prime 2} ; \quad\left\{\begin{array}{l}
\frac{\partial^{\prime} E^{\prime 3}}{\partial^{\prime} v}=B^{\prime 2}, \\
\frac{\partial^{\prime} B^{\prime 2}}{\partial^{\prime} v}=\frac{\varepsilon \mu}{c^{2}} E^{\prime 3} ;
\end{array} \quad \frac{\partial^{\prime} E^{\prime 1}}{\partial^{\prime} v}=0 ; \frac{\partial^{\prime} B^{\prime 1}}{\partial^{\prime} v}=0 .\right.
\end{array}\right.
$$

Продифференцируем первые уравнения систем (17.74) и подставим их во вторые уравнения: 


$$
\frac{\partial^{\prime 2} E^{\prime 2}}{\partial^{\prime} v^{2}}=\frac{\varepsilon \mu}{c^{2}} E^{\prime 2} ; \frac{\partial^{\prime 2} E^{\prime 3}}{\partial^{\prime} v^{2}}=\frac{\varepsilon \mu}{c^{2}} E^{\prime 3} ; \frac{\partial^{\prime 2} B^{\prime 2}}{\partial^{\prime} v^{2}}=\frac{\varepsilon \mu}{c^{2}} B^{\prime 2} ; \frac{\partial^{\prime 2} B^{\prime 3}}{\partial^{\prime} v^{2}}=\frac{\varepsilon \mu}{c^{2}} B^{\prime 3} .
$$

Общее решение уравнений (17.75) выражается через произвольные постоянные $C_{1}, \ldots, C_{10}$ :

$$
\begin{gathered}
E^{\prime 1}=C_{1} ; \quad E^{\prime 2}=C_{2} \cosh \frac{\sqrt{\varepsilon \mu \nu}}{c}+C_{3} \sinh \frac{\sqrt{\varepsilon \mu \nu}}{c} ; \\
E^{\prime 3}=C_{4} \cosh \frac{\sqrt{\varepsilon \mu \nu} v}{c}+C_{5} \sinh \frac{\sqrt{\varepsilon \mu \nu}}{c} ; \\
B^{\prime 1}=C_{6} ; \quad B^{\prime 2}=C_{7} \cosh \frac{\sqrt{\varepsilon \mu \nu}}{c}+C_{8} \sinh \frac{\sqrt{\varepsilon \mu \nu}}{c} \\
B^{\prime 3}=C_{9} \cosh \frac{\sqrt{\varepsilon \mu} v}{c}+C_{10} \sinh \frac{\sqrt{\varepsilon \mu} v}{c}
\end{gathered}
$$

Так как мы ищем преобразования электромагнитного поля при переходе от лабораторной системы отсчета, то искомые частные решения уравнений (17.75) должны при $v=0$ описывать электромагнитное поле в лабораторной системе отсчета, то есть удовлетворять равенствам (17.8) и (17.74), а, значит, следующей совокупности равенств:

$$
\begin{gathered}
E^{\prime 1}\left(0, \mathbf{r}^{\prime}, t\right)=E^{1}\left(\mathbf{r}^{\prime}, t\right) ; E^{\prime 2}\left(0, \mathbf{r}^{\prime}, t\right)=E^{2}\left(\mathbf{r}^{\prime}, t\right) ; E^{\prime 3}\left(0, \mathbf{r}^{\prime}, t\right)=E^{3}\left(\mathbf{r}^{\prime}, t\right) ; \\
B^{\prime 1}\left(0, \mathbf{r}^{\prime}, t\right)=B^{1}\left(\mathbf{r}^{\prime}, t\right) ; B^{\prime 2}\left(0, \mathbf{r}^{\prime}, t\right)=B^{2}\left(\mathbf{r}^{\prime}, t\right) ; B^{\prime 3}\left(0, \mathbf{r}^{\prime}, t\right)=B^{3}\left(\mathbf{r}^{\prime}, t\right) ; \\
\frac{\partial^{\prime} E^{\prime 2}\left(0, \mathbf{r}^{\prime}, t\right)}{\partial^{\prime} v}=-B^{3}\left(\mathbf{r}^{\prime}, t\right) ; \frac{\partial^{\prime} E^{\prime 3}\left(0, \mathbf{r}^{\prime}, t\right)}{\partial^{\prime} v}=B^{2}\left(\mathbf{r}^{\prime}, t\right) ; \\
\frac{\partial^{\prime} B^{\prime 2}\left(0, \mathbf{r}^{\prime}, t\right)}{\partial^{\prime} v}=\frac{\varepsilon \mu}{c^{2}} E^{3}\left(\mathbf{r}^{\prime}, t\right) ; \frac{\partial^{\prime} B^{\prime 3}\left(0, \mathbf{r}^{\prime}, t\right)}{\partial^{\prime} v}=-\frac{\varepsilon \mu}{c^{2}} E^{2}\left(\mathbf{r}^{\prime}, t\right)
\end{gathered}
$$

Подстановкой (17.76), (17.77) в (17.78)-(17.81) найдем значения постоянных $C_{1}, \ldots, C_{10}$, в результате чего после подстановки этих постоянных в (17.76), (17.77) получим окончательное выражение в компонентной форме для искомых преобразований электромагнитного поля при переходе от лабораторной системы отсчета к субстанциональной:

$$
\begin{gathered}
E^{\prime}\left(v, \mathbf{r}^{\prime}, t\right)=E^{1}\left(\mathbf{r}^{\prime}, t\right) ; B^{\prime 1}\left(v, \mathbf{r}^{\prime}, t\right)=B^{1}\left(\mathbf{r}^{\prime}, t\right) \\
E^{\prime 2}\left(v, \mathbf{r}^{\prime}, t\right)=E^{2}\left(\mathbf{r}^{\prime}, t\right) \cosh \frac{\sqrt{\varepsilon \mu} v}{c}-\frac{c}{\sqrt{\varepsilon \mu}} B^{3}\left(\mathbf{r}^{\prime}, t\right) \sinh \frac{\sqrt{\varepsilon \mu} v}{c} \\
E^{\prime 3}\left(v, \mathbf{r}^{\prime}, t\right)=E^{3}\left(\mathbf{r}^{\prime}, t\right) \cosh \frac{\sqrt{\varepsilon \mu} v}{c}+\frac{c}{\sqrt{\varepsilon \mu}} B^{2}\left(\mathbf{r}^{\prime}, t\right) \sinh \frac{\sqrt{\varepsilon \mu \nu}}{c} \\
B^{\prime 2}\left(v, \mathbf{r}^{\prime}, t\right)=B^{2}\left(\mathbf{r}^{\prime}, t\right) \cosh \frac{\sqrt{\varepsilon \mu} v}{c}+\frac{\sqrt{\varepsilon \mu}}{c} E^{3}\left(\mathbf{r}^{\prime}, t\right) \sinh \frac{\sqrt{\varepsilon \mu} v}{c} \\
B^{\prime 3}\left(v, \mathbf{r}^{\prime}, t\right)=B^{3}\left(\mathbf{r}^{\prime}, t\right) \cosh \frac{\sqrt{\varepsilon \mu \nu}}{c}-\frac{\sqrt{\varepsilon \mu}}{c} E^{2}\left(\mathbf{r}^{\prime}, t\right) \sinh \frac{\sqrt{\varepsilon \mu \nu}}{c}
\end{gathered}
$$

В векторной форме те же самые преобразования имеют следующий вид: 


$$
\begin{aligned}
& \mathbf{E}^{\prime}\left(v, \mathbf{r}^{\prime}, t\right)=\mathbf{E}\left(\mathbf{r}^{\prime}, t\right) \cosh \frac{\sqrt{\varepsilon \mu} v}{c}+\frac{c}{\sqrt{\varepsilon \mu}} \mathbf{e}_{1} \times \mathbf{B}\left(\mathbf{r}^{\prime}, t\right) \sinh \frac{\sqrt{\varepsilon \mu \nu}}{c} \\
& \mathbf{E}^{\prime}\left(v, \mathbf{r}^{\prime}, t\right)=\mathbf{E}\left(\mathbf{r}^{\prime}, t\right) \cosh \frac{\sqrt{\varepsilon \mu} v}{c}+\frac{c}{\sqrt{\varepsilon \mu}} \mathbf{e}_{1} \times \mathbf{B}\left(\mathbf{r}^{\prime}, t\right) \sinh \frac{\sqrt{\varepsilon \mu \nu}}{c} .
\end{aligned}
$$

Легко видеть, что (17.82-17.88) есть известные преобразования Менде [58]. Они получают достаточное теоретическое обоснование в рамках транскоординатной формулировки электродинамики, связанной с гиперконтинуальными представлениями о пространстве и времени, а также с концепцией неинвариантности электрического заряда относительно скорости движения наблюдателя. Наряду с прямым экспериментальным подтверждением в [50] концепции неинвариантности электрического заряда, это убедительно свидетельствует об их большей адекватности физической реальности по сравнению не только с классическими, но и с релятивистскими преобразованиями электромагнитного поля, а также об оправданности перевода электродинамики с традиционной формулировки ГерцаХевисайда на транскоординатную. Последовательное развитие транскоординатной электродинамики способно не только вывести на новый качественный уровень представления о пространстве и времени, но и открыть принципиально новые горизонты развития техники и технологий за счет открытия и освоения новых физических явлений и эффектов.

\section{§ 18. Электрические поля в концепции скалярно-векторного потенциала}

В концепции скалярно-векторного потенциала можно выделить, по крайней мере, три случая зависимости напряженности электрического поля от скорости заряда, характеризующие разные варианты этой концепции.

\section{1) Непотенциальное электрическое поле.}

Если речь идет об электрическом поле одиночного заряда $e$, то его электрическое поле будет определяться соотношением:

$$
\mathbf{E}=\frac{e}{4 \pi \varepsilon_{0} \mathbf{r}^{2}}\left(\mathbf{e}_{v}+\operatorname{ch} \frac{\sqrt{\mathbf{v}^{2}}}{c} \mathbf{e}_{\perp}\right)
$$

где $\mathbf{e}_{v}=\frac{\mathbf{r} \cdot \mathbf{v}}{\mathbf{v}^{2} \sqrt{\mathbf{r}^{2}}} \mathbf{v}-$ проекция на направление вектора $\mathbf{v}$ единичного вектора $\mathbf{e}_{r}=\mathbf{r} / \sqrt{\mathbf{r}^{2}}$, коллинеарного вектору $\mathbf{r} ; \mathbf{e}_{\perp}=\mathbf{e}_{r}-\mathbf{e}_{v}=\frac{\mathbf{r}}{\sqrt{\mathbf{r}^{2}}}-\frac{\mathbf{r} \cdot \mathbf{v}}{\mathbf{v}^{2} \sqrt{\mathbf{r}^{2}}} \mathbf{v}-$ проекция единичного вектора $\mathbf{e}_{r}=\mathbf{r} / \sqrt{\mathbf{r}^{2}}$, коллинеарного вектору $\mathbf{r}$, на направление нормали к вектору $\mathbf{v}$, лежащей в одной плоскости с $\mathbf{r}$.

Зафиксируем некоторый момент времени. Выберем систему прямоугольных декартовых пространственных координат $O X Y Z$ так, чтобы в этот мо- 
мент времени начало координат совпадало с движущимся точечным зарядом $e$, ось $O X$ была направлена вдоль вектора скорости заряда $\mathbf{v}$, а вектор $\mathbf{r}$ лежал в плоскости $X O Y$. Тогда вектор $\mathbf{r}$ можно задавать двумя координатами $x$ и $y$ по осям $O X$ и $O Y$ соответственно. В этом случае продольная (по оси $O X$ ) и поперечная (по оси $O Y$ ) компоненты вектора напряженности электрического поля в точке $\mathbf{r}$ будут равны соответственно

$$
E_{x}=\frac{e}{4 \pi \varepsilon_{0}} \cdot \frac{x}{\sqrt{\left(x^{2}+y^{2}\right)^{3}}} ; E_{y}=\frac{e}{4 \pi \varepsilon_{0}} \operatorname{ch} \frac{\sqrt{\mathbf{v}^{2}}}{c} \cdot \frac{y}{\sqrt{\left(x^{2}+y^{2}\right)^{3}}} .
$$

Интегрирование этих компонент по соответствующим координатам дает:

$$
\int E_{x} d x=-\frac{e}{4 \pi \varepsilon_{0}} \cdot \frac{1}{\sqrt{x^{2}+y^{2}}}+C_{1}(y) ; \int E_{y} d y=-\frac{e}{4 \pi \varepsilon_{0}} \operatorname{ch} \frac{\sqrt{\mathbf{v}^{2}}}{c} \cdot \frac{1}{\sqrt{x^{2}+y^{2}}}+C_{2}(x),
$$

где $C_{1}(y)$ и $C_{2}(x)$ - постоянные интегрирования.

При $\sqrt{\mathbf{v}^{2}}=0$ электрическое поле оказывается потенциальным с известным потенциалом

$$
\varphi(x, y)=\frac{e}{4 \pi \varepsilon_{0}} \cdot \frac{1}{\sqrt{x^{2}+y^{2}}}
$$

но при $\sqrt{\mathbf{v}^{2}} \neq 0$ потенциальность поля нарушается, то есть не существует такой функции потенциала, через которую можно было бы выразить напряженность поля по формуле

$$
\mathbf{E}=-\nabla \varphi
$$

Поэтому такое электрическое поле движущегося заряда нужно рассматривать, как суперпозицию

$$
\mathbf{E}=\mathbf{E}_{v}+\mathbf{E}_{\perp}
$$

продольного электрического поля $\mathbf{E}_{v}$ с потенциалом (назовем его продольным потенциалом)

$$
\varphi_{v}(x, y)=\frac{e}{4 \pi \varepsilon_{0}} \cdot \frac{1}{\sqrt{x^{2}+y^{2}}}
$$

и поперечного электрического поля $\mathbf{E}_{\perp}$ с потенциалом (назовем его поперечным потенциалом)

$$
\varphi_{\perp}(x, y)=\frac{e}{4 \pi \varepsilon_{0}} \operatorname{ch} \frac{\sqrt{\mathbf{v}^{2}}}{c} \cdot \frac{1}{\sqrt{x^{2}+y^{2}}},
$$

причем поля $\mathbf{E}_{v}$ и $\mathbf{E}_{\perp}$ имеют ненулевые компоненты только по $O X$ и $O Y$ :

$$
\mathbf{E}_{v}=\frac{\partial \varphi_{v}}{\partial x} ; \mathbf{E}_{\perp}=\frac{\partial \varphi_{\perp}}{\partial y}
$$

Поэтому в данном случае более правильно говорить не о концепции скалярного потенциала заряда, зависящего от скорости, а о концепции инвариантного продольного скалярного потенциала и зависящего от скорости поперечного скалярного потенциала. 


\section{2) Потенциальное электрическое поле Менде.}

Зафиксируем некоторый момент времени. Выберем систему прямоугольных декартовых пространственных координат $O X Y Z$ так, чтобы в этот момент времени начало координат совпадало с движущимся точечным зарядом $e$, ось $O Y$ была направлена вдоль вектора скорости заряда $\mathbf{v}$, а вектор $\mathbf{r}$, направленный от точки сосредоточения заряда к точке, в которой определяется поле, лежал в плоскости XOY. Тогда вектор $\mathbf{r}$ можно задавать двумя координатами $x$ и $y$ по осям $O X$ и $O Y$ соответственно.

Предположим, что электрическое поле является потенциальным полем, задаваемым скалярным потенциалом

$$
\varphi(\mathbf{r})=e \operatorname{ch}\left(v_{\perp}(\mathbf{r}) / c\right) /\left(4 \pi \varepsilon_{0} r\right)
$$

где $\mathbf{r}=(x, y)$ - радиус-вектор точки, в которой определяется поле; $r=\sqrt{\mathbf{r}^{2}}=\sqrt{x^{2}+y^{2}}-$ расстояние между зарядом и точкой, в которой определяется поле; $v_{\perp}(\mathbf{r})$ - составляющая скорости заряда $e$, нормальная к $\mathbf{r}$.

Пусть $x>0, y>0$, тогда имеем:

$$
v_{\perp}(x, y)=\frac{v x}{\sqrt{x^{2}+y^{2}}}=\frac{v x}{r} ; \varphi(x, y)=\frac{e}{4 \pi \varepsilon_{0} \sqrt{x^{2}+y^{2}}} \operatorname{ch} \frac{v x}{c \sqrt{x^{2}+y^{2}}}=\frac{e}{4 \pi \varepsilon_{0} r} \operatorname{ch} \frac{v x}{c r} .
$$

Напряженность такого поля имеет поперечную компоненту $E_{x}(x, y)$ и продольную компоненту $E_{y}(x, y)$, которые определяются по формулам:

$$
\begin{gathered}
E_{x}(x, y)=-\frac{\partial \varphi(x, y)}{\partial x}=\frac{e}{4 \pi \varepsilon_{0} r^{3}}\left(x \operatorname{ch} \frac{v x}{c r}-\frac{v y^{2}}{c r} \operatorname{sh} \frac{v x}{c r}\right) \\
E_{y}(x, y)=-\frac{\partial \varphi(x, y)}{\partial y}=\frac{e y}{4 \pi \varepsilon_{0} r^{3}}\left(\operatorname{ch} \frac{v x}{c r}-\frac{v x}{c r} \operatorname{sh} \frac{v x}{c r}\right) .
\end{gathered}
$$

\section{3) Потенциальное электрическое поле с поперечно деформирован- ным скалярным потенциалом.}

Выберем систему прямоугольных декартовых пространственных координат так, как в случае 2. Электростатический потенциал заряда имеет вид:

$$
\varphi(x, y)=e /\left(4 \pi \varepsilon_{0} r\right)=e /\left(4 \pi \varepsilon_{0} \sqrt{x^{2}+y^{2}}\right)
$$

где $\mathbf{r}=(x, y)$ - радиус-вектор точки, в которой определяется поле; $r=\sqrt{\mathbf{r}^{2}}=\sqrt{x^{2}+y^{2}}-$ расстояние между зарядом и этой точкой.

Положим, что динамический потенциал заряда есть поперечно деформированный электростатический потенциал. Тогда получаем потенциальное электрическое поле, описываемое скалярным потенциалом

$$
\varphi_{\perp}(v, x, y)=\varphi(x / \operatorname{ch}(v / c), y)
$$

где $v=\sqrt{\mathbf{v}^{2}}$ - модуль скорости заряда, причем 


$$
\varphi_{\perp}(0, x, y)=\varphi(x, y) .
$$

Учитывая выражение для электростатического потенциала, получаем формулу для динамического потенциала:

$$
\varphi_{\perp}(v, x, y)=\frac{e \operatorname{ch}(v / c)}{4 \pi \varepsilon_{0} \sqrt{x^{2}+y^{2} \operatorname{ch}^{2}(v / c)}} .
$$

Поперечная $E_{\perp x}(v, x, y)$ и продольная $E_{\perp y}(v, x, y)$ компоненты напряженности поля запишутся в виде

$$
\begin{aligned}
& E_{\perp x}(v, x, y)=-\frac{\partial \varphi_{\perp}(v, x, y)}{\partial x}=\frac{\operatorname{exch}(v / c)}{4 \pi \varepsilon_{0} \sqrt{\left(x^{2}+y^{2} \operatorname{ch}^{2}(v / c)\right)^{3}}} \\
& E_{\perp y}(v, x, y)=-\frac{\partial \varphi_{\perp}(v, x, y)}{\partial y}=\frac{e y \operatorname{ch}^{3}(v / c)}{4 \pi \varepsilon_{0} \sqrt{\left(x^{2}+y^{2} \operatorname{ch}^{2}(v / c)\right)^{3}}} .
\end{aligned}
$$

При $x \neq 0, y=0$ имеем:

$$
E_{\perp x}(v, x, y)=\frac{e \operatorname{ch}(v / c)}{4 \pi \varepsilon_{0} x^{2}} ; E_{\perp y}(v, x, y)=0 .
$$

При $x=0, y \neq 0$ имеем:

$$
E_{\perp x}(v, x, y)=0 ; E_{\perp y}(v, x, y)=\frac{e}{4 \pi \varepsilon_{0} y^{2}} .
$$

\section{Выводы из трех рассмотренных случаев следующие.}

Таким образом, предложено три варианта определения электрических полей в концепции скалярно-векторного потенциала:

1) непотенциальное поле, у которого продольная компонента напряженности не зависит от скорости, а поперечная - зависит;

2) потенциальное поле в варианте Менде;

3) потенциальное поле с поперечно деформированным скалярным потенциалом.

Последние два варианта характеризуют потенциальное поле, у которого в общем случае обе компоненты зависят от скорости; однако для этого поля можно выделить два крайних частных случая для точек, в которых это поле определяется: если в какой-то точке (в предложенной системе координат эти точки лежат на оси $O Y$ ) поперечная компонента поля отсутствует, то продольная не зависит от скорости; если в какой-то точке (в предложенной системе координат эти точки лежат на оси $O X$ ) продольная компонента поля отсутствует, то поперечная зависит от скорости простой зависимостью, получающейся умножением соответствующей напряженности электростатического поля на коэффициент $\operatorname{ch}(v / c)$.

Потенциальность электрического поля означает, что при движении в этом поле пробного заряда по замкнутой траектории работа не совершается. Если же скалярный потенциал изменяется во времени, то во времени 
меняется и электрическое поле, в результате чего данное свойство отсутствия совершенной работы, строго говоря, не выполняется. Однако и в этом случае для каждого заданного момента времени потенциальное электрическое поле (последние два варианта) может быть определено как взятый с обратным знаком градиент скалярного потенциала.

Важное отличие первого и третьего вариантов состоит в том, что в первом варианте имеет место поперечная деформация напряженности поля (она приводит к непотенциальности поля), а в третьем - поперечная деформация скалярного потенциала поля (она приводит к зависимости от скорости в общем случае обоих компонент напряженности поля).

В настоящей монографии за основу взят второй вариант концепции, хотя третий вариант с физической точки зрения представляется нам не менее, a, возможно, более перспективным, и первый вариант тоже достаточно перспективен. В связи с этим, одна из важнейших задач дальнейших исследований - определение варианта, наиболее адекватного реальности.

\section{Закон электро-электрической индукции.}

Известный задолго до появления уравнений Максвелла закон Ампера в векторной форме определяет магнитное поле в данной точке:

$$
\mathbf{H}=\frac{1}{4 \pi} \int \frac{I d \mathbf{l} \times \mathbf{r}}{r^{3}},
$$

где $I$ - ток в элементе $d \mathbf{l} ; \mathbf{r}$ - вектор, направленный из $d \mathbf{l}$ в данную точку.

Можно показать, что

$$
\frac{d \mathbf{l} \times \mathbf{r}}{r^{3}}=\operatorname{grad}\left(\frac{1}{r}\right) \times d \mathbf{l}=\operatorname{rot}\left(\frac{d \mathbf{l}}{r}\right)-\frac{1}{r} \operatorname{rot} d \mathbf{l}=\operatorname{rot}\left(\frac{d \mathbf{l}}{r}\right),
$$

поэтому окончательно получим:

$$
\mathbf{H}=\operatorname{rot} \int I\left(\frac{d \mathbf{l}}{4 \pi r}\right)=\operatorname{rot} \mathbf{A}_{\mathbf{H}},
$$

где

$$
\mathbf{A}_{\mathbf{H}}=\int I\left(\frac{d \mathbf{l}}{4 \pi r}\right)
$$

Замечательным свойством этого выражения является то, что векторный потенциал зависит от расстояния до точки наблюдения как $1 / r$. Именно это свойство и позволяет получить законы излучения.

Т.к. $I=g v$, где $g$ - заряд единицы длины проводника, из (18.1) имеем:

$$
\mathbf{A}_{\mathbf{H}}=\int \frac{g v d \mathbf{l}}{4 \pi r} .
$$

Для одиночного заряда $е$ это соотношение принимает вид:

$$
\mathbf{A}_{\mathbf{H}}=\frac{e \mathbf{v}}{4 \pi r} .
$$

Поскольку 


$$
\mathbf{E}=-\mu \frac{\partial \mathbf{A}_{\mathbf{H}}}{\partial t},
$$

то в общем случае имеем (здесь $a$ - ускорение заряда):

$$
\mathbf{E}=-\mu \int \frac{g \frac{\partial v}{\partial t} d \mathbf{l}}{4 \pi r}=-\mu \int \frac{g a d \mathbf{l}}{4 \pi r},
$$

а для частного случая одиночного заряда получим:

$$
\mathbf{E}=-\frac{\mu e \mathbf{a}}{4 \pi r} \text {. }
$$

Если в (18.2), (18.3) учесть запаздывание $(t-r / c)$ распространения потенциалов и то, что для вакуума $\mu=1 /\left(\varepsilon_{0} c^{2}\right)$, эти равенства примут вид:

$$
\begin{gathered}
\mathbf{E}=-\mu \int \frac{g a(t-r / c) d \mathbf{l}}{4 \pi r}=-\int \frac{g a(t-r / c) d \mathbf{l}}{4 \pi \varepsilon_{0} c^{2} r}, \\
\mathbf{E}=-\frac{e \mathbf{a}(t-r / c)}{4 \pi \varepsilon_{0} c^{2} r} .
\end{gathered}
$$

Волновые уравнения (18.4), (18.5) здесь получены с использованием понятий магнитного поля и его векторного потенциала, но сами по себе они лишь дают зависимость электрического поля от индуцирующих его движущихся зарядов. Знание такой зависимости позволяет при описании процессов излучения вообще не использовать понятий магнитного поля и его векторного потенциала.

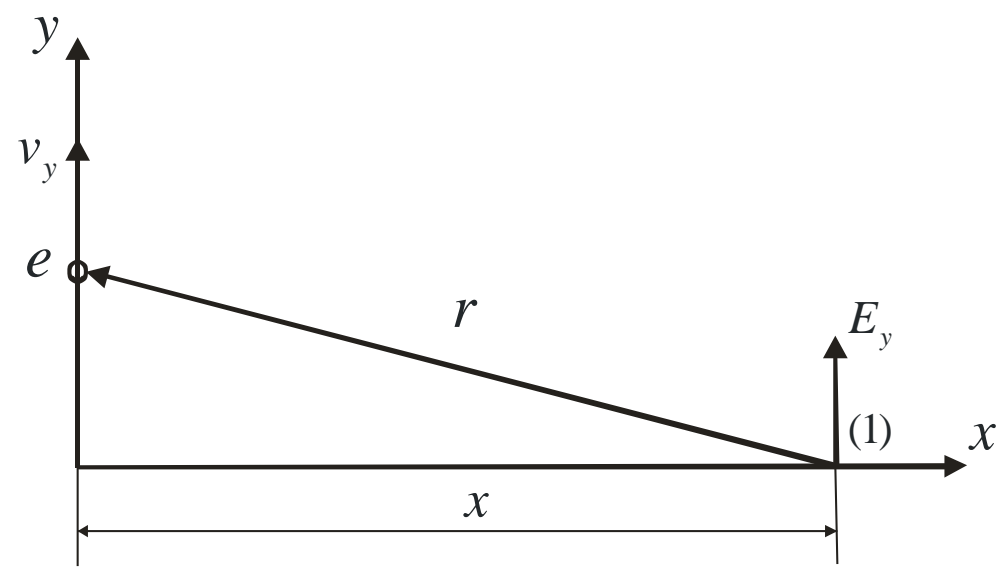

Рис. 13. Схема формирования индуцированного электрического поля.

Пусть заряд $e$ колеблется вдоль оси $y$ в окрестности точки 0 с амплитудой, значительно меньшей расстояния $r$ от наблюдаемой точки (1) с $y=0$ до заряда (рис. 13). Амплитудные и фазовые характеристики излучаемой зарядом волны электрического поля определяются волновым уравнением

$$
E_{y}(x, t)=-\frac{e}{4 \pi \varepsilon_{0} c^{2} x} \frac{\partial v_{y}(t-x / c)}{\partial t}=-\frac{e a_{y}(t-x / c)}{4 \pi \varepsilon_{0} c^{2} x},
$$


где $v_{y}(t-x / c), a_{y}(t-x / c)$ - запаздывающие скорость и ускорение заряда.

Выбрав направлением излучения вектор в плоскости $x y$, составляющий с осью $y$ угол $\alpha$, из (18.6) получим соотношение, определяющее известную диаграмму направленности дипольного излучателя (полная диаграмма симметрична относительно оси $y$ ):

$$
E_{y}(x, t, \alpha)=-\frac{e a_{y}(t-x / c) \sin \alpha}{4 \pi \varepsilon_{0} c^{2} x}=-\frac{1}{\varepsilon_{0} c^{2}} \frac{\partial A_{H}(t-x / c)}{\partial t}=-\mu_{0} \frac{\partial A_{H}(t-x / c)}{\partial t} .
$$

Известный в электродинамике запаздывающий векторный потенциал

$$
\frac{e v_{y}(t-x / c)}{4 \pi x}=A_{H}(t-x / c)
$$

введен на основе закона электро-электрической индукции. Электрические поля волны градиентные, а не вихревые, как в уравнениях Максвелла.

С помощью (18.7) можно описать дипольное излучение зарядов, колеблющихся в электрическом поле. Производная по времени дипольного момента (вектор d направлен от отрицательного заряда к положительному) $\mathbf{p}=e \mathbf{d}$ связана с током:

$$
e \mathbf{v}=e \frac{\partial \mathbf{d}}{\partial t}=\frac{\partial \mathbf{p}}{\partial t}, \mathbf{v}=\frac{1}{e} \frac{\partial \mathbf{p}}{\partial t}, \mathbf{a}=\frac{\partial \mathbf{v}}{\partial t}=\frac{1}{e} \frac{\partial^{2} \mathbf{p}}{\partial t^{2}}
$$

Подстановка последнего равенства для а в (18.7) дает известный [1] закон излучения колеблющегося диполя

$$
\mathbf{E}=-\frac{1}{4 \pi r \varepsilon_{0} c^{2}} \frac{\partial^{2} \mathbf{p}(t-r / c)}{\partial t^{2}}
$$

Таким образом, поле колеблющегося электрического диполя определяется суперпозицией электрических индукционных полей излучения (18.6)(18.8), связанных с ускорением заряда, и электрических полей статического диполя, изменяющихся согласно зависимости от времени расстояния между зарядами. Процесс излучения связан с превращением энергии полей статического диполя в энергию полей излучения.

Отсутствие в (18.6)-(18.8) магнитных полей и векторных потенциалов подтверждает фундаментальность электрических полей.

Соотношение (18.7) позволяет получить законы отражения и рассеивания электрических полей совокупностью зарядов, приводимых в вынужденное движение внешним (сторонним) электрическим полем. Суперпозиция излучения электрических полей всеми зарядами есть электрическая волна.

Ускорение заряда под действием стороннего электрического поля $E_{y}^{\prime}=E_{y 0}^{\prime} \sin \omega t$ равно

$$
a=-\frac{e}{m} E_{y 0}^{\prime} \sin \omega t
$$

С учётом этого, соотношение (18.7) принимает вид:

$$
E_{y}(x, t, \alpha)=\frac{e^{2} \sin \alpha}{4 \pi \varepsilon_{0} c^{2} m x} E_{y 0}^{\prime} \sin \omega\left(t-\frac{x}{c}\right)=\frac{K}{x} E_{y 0}^{\prime} \sin \omega\left(t-\frac{x}{c}\right),
$$


где определяющую способность заряда переизлучать внешнее поле величину

$$
K=\frac{e^{2} \sin \alpha}{4 \pi \varepsilon_{0} c^{2} m}
$$

назовем коэффициентом рассеивания (переизлучения) заряда в заданном направлении.

Волне электрического поля (18.7) сопутствует волна тока смещения

$$
j_{y}(x, t)=\varepsilon_{0} \frac{\partial E_{y}}{\partial t}=-\frac{e \sin \alpha}{4 \pi c^{2} x} \frac{\partial^{2} v_{y}(t-x / c)}{\partial t^{2}} .
$$

Если заряд осуществляет своё движение под воздействием стороннего электрического поля $E^{\prime}=E_{0}^{\prime} \sin \omega t$, то ток смещения в дальней зоне равен

$$
j_{y}(x, t)=-\frac{e^{2} \omega}{4 \pi c^{2} m x} E_{y 0}^{\prime} \cos \omega(t-x / c) .
$$

Волна электрического поля (18.9) и отстающая от неё на $\pi / 2$ волна токов смещения (18.10) образуют волну, впервые описанную в $[11,13]$ и названную там электротоковой.

Параллельно с электрическими волнами введем магнитные, положив

$$
\begin{aligned}
\mathbf{j}=\varepsilon_{0} \frac{\partial \mathbf{E}}{\partial t} & =\operatorname{rot} \mathbf{H}, \\
\operatorname{div} \mathbf{H} & =0 .
\end{aligned}
$$

Такое магнитное поле - вихревое. Сравнивая (18.10) и (18.11), получаем:

$$
\frac{\partial H_{z}(x, t)}{\partial x}=\frac{e^{2} \omega \sin \alpha}{4 \pi c^{2} m x} E_{y 0}^{\prime} \cos \omega\left(t-\frac{x}{c}\right) .
$$

Интегрируя это соотношение по координате, находим магнитное поле

$$
H_{z}(x, t)=\frac{e^{2} \sin \alpha}{4 \pi c m x} E_{y 0}^{\prime} \sin \omega\left(t-\frac{x}{c}\right) .
$$

Соотношения (18.9), (18.10) и (18.12) могут быть названы законами электро-электрической индукции, т.к. дают непосредственную связь между прикладываемыми к заряду сторонними электрическими полями и токами, индуцируемыми этим зарядом в его окрестности. Заряд выступает в роли трансформатора, обеспечивающего переизлучение. Магнитное поле (18.12) направлено нормально и к электрическому полю, и к направлению распространения. При этом выполнено:

$$
\frac{E_{y}(x, t)}{H_{z}(x, t)}=\frac{1}{\varepsilon_{0} c}=\sqrt{\frac{\mu_{0}}{\varepsilon_{0}}}=Z,
$$

где $Z$ - волновое сопротивление свободного пространства.

Волновое сопротивление определяет активную мощность потерь на единичной площадке, нормальной к направлению распространения волны:

$$
P=Z E_{y 0}^{2} / 2 \text {. }
$$

Данное соотношение определяет поток мощности электротоковой волны через такую площадку согласно теореме Пойнтинга. 
Таким образом, любой волновой процесс в полях электромагнитной природы сводится к электротоковым волнам в пространстве, характеризуемом своим волновым сопротивлением. Такое рассмотрение не требует привлечения понятий магнитного поля и его векторного потенциала. Тем не менее, эти понятия можно дополнительно вводить, чётко разделяя поля на градиентные (электрические) и вихревые (магнитные) согласно теореме Гельмгольца, гласящей, что всякое однозначное и непрерывное векторное поле $\mathbf{F}$, обращающееся в ноль на бесконечности, может быть представлено, и притом единственным образом, в виде суммы градиента некоторой скалярной функции $\varphi$ и ротора некоторой векторной функции $\mathbf{C}$, дивергенция которой равна нулю:

$$
\mathbf{F}=\operatorname{grad} \varphi+\operatorname{rot} \mathbf{C}, \operatorname{div} \mathbf{C}=0 .
$$

Металлические опилки, выстраивающиеся вокруг полюсов магнита, указывают на наличие каких-то силовых полей потенциального характера, но в своё время из этого не сделали должных выводов, и значительная «подводная часть айсберга» оставалась невидимой почти двести лет.

Таким образом, построение электродинамики нужно было начинать с признания зависимости электрического поля заряда от параметров его движения, как это предлагали многие ведущие учёные того времени. Но природа очень глубоко прячет свои секреты, и, чтобы вернуться к такому простому выводу, пришлось пройти путь длиной почти в два столетия.

Показательна цитата из [3]: «в чем же заключается основная исходная причина противоречивости построенной Максвеллом электродинамики? Для однозначного ответа на этот вопрос... следует отметить, что еще в свое время Ампер, Гроссман, Гаусс, Ленц, Нейман, Вебер, Риман и др. стояли на точке зрения, что, не обращаясь к понятию «магнитного поля», любые магнитные взаимодействия можно свести к обычным взаимодействиям токовых элементов или движущихся зарядов... в электродинамике возобладала тогда точка зрения Фарадея и Максвелла, что электрические и «магнитные» поля являются самостоятельными физическими сущностями, хотя и связанными между собой. В сложившейся тогда исторической обстановке данные, ошибочные с физической точки зрения, допущения предопределили собой весь дальнейший ход развития электродинамики с заведомо заложенными в нее неразрешимыми противоречиями и парадоксами». И далее там же: «для непротиворечивого отражения физической сущности законов электромагнетизма необходимо полностью отказаться от любых понятий «магнитного поля» как некой самостоятельной физической сущности... для определения сил взаимодействия движущихся в физическом вакууме реального пространства электрических зарядов вполне достаточно учесть деформацию электрических полей этих зарядов, обусловленную тривиальными эффектами запаздывающих потенциалов... Остается только удивляться прозорливости Ампера, который предупреждал, что если в электродинамике не отказаться от понятия «магнит», то в дальнейшем это грозит неимоверной путаницей в теории». 
Вот как мало продвинулась электродинамика за всю свою историю. Явление электромагнитной индукции Фарадей открыл в 1831 г., и уже почти 200 лет его учение остаётся практически неизменным, а физические причины многих электродинамических явлений - не понятыми. Фарадей был гением своего времени, но что делали физики после него? Даже гениальные Максвелл и Герц не установили фундаментальную роль зависимости электрического поля заряда от параметров его движения. Впоследствии такие ученые, как Николаев Г.В. и Маринов С. проводили в этом направлении свои теоретические и экспериментальные исследования, но должного признания научным сообществом эти работы так и не получили.

Теперь можно снять «строительные леса» в виде магнитного поля и векторного потенциала, которые не позволяют вот уже почти двести лет увидеть здание электродинамики во всём его величии и красоте.

Следует полагать, что в основе электродинамики нужно положить три закона: закон Кулона, закон зависимости электрического поля заряда от скорости его движения и закон зависимости электрического поля заряда от его ускорения. Закон Кулона определяет электрическое поле неподвижного заряда. Наличие у заряда скорости приводит к приращению его электрического поля в поперечном относительно скорости направлении. А ускорение заряда приводит к индуцированию дополнительного электрического поля в продольном относительно ускорения направлении. Закон Кулона и закон зависимости электрического поля заряда от его ускорения уже содержатся в электродинамике Максвелла. Принципиально новым же является закон зависимости электрического поля заряда от скорости его движения, реализующий концепцию скалярно-векторного потенциала. Объединение всех этих трёх законов в единый фундамент электродинамики коренным образом меняет её идеологию. Оно позволяет рассматривать с единых позиций и статическое взаимодействие зарядов, и законы силового взаимодействия в случае их взаимного движения, и законы излучения и рассеивания. Такой подход позволил преодолеть описанные в данной монографии теоретические проблемы электродинамики, объяснить без привлечения СТО фазовую аберрацию и поперечный эффект Доплера, что не удавалось сделать в рамках традиционной идеологии электродинамики.

\section{§ 19. Силовое взаимодействие токонесущих систем, униполярная индукция и пондеромоторные силы}

Уже было сказано, что уравнения Максвелла не содержат в себе информации о силовом взаимодействии токонесущих систем. В классической электродинамике для расчета такого взаимодействия нужно рассчитать магнитное поле в заданной области пространства, а затем, используя силу Лоренца, вводимую как отдельный экспериментальный постулат, находить силы, действующие на заряды, движущиеся в заданном поле. При таком 
подходе неясным остается вопрос о том, к чему приложены силы реакции по отношению к тем силам, которые действуют на движущиеся заряды.

Концепция магнитного поля возникла, в значительной степени, благодаря наблюдениям за силовым взаимодействием токонесущих и намагниченных систем. Особенно показателен опыт с железными опилками, которые выстраиваются около полюсов магнита или вокруг кольцевого витка с током в чёткие геометрические фигуры. Эти фигуры послужили поводом для введения понятия силовых линий. Согласно третьему закону Ньютона, при любом силовом взаимодействии силы действия равны силам противодействия, причём всегда имеются те элементы системы, к которым эти силы приложены. Большим недостатком концепции магнитного поля является то, что она не определяет конкретные места приложения сил противодействия, т.к. магнитное поле выступает как самостоятельная субстанция, с которой взаимодействуют движущиеся заряды.

Экспериментально известно, что силы взаимодействия в токонесущих системах приложены к проводникам, движущиеся заряды которых создают магнитное поле. Однако в концепции магнитного поля и силы Лоренца положительно заряженная решетка, являющаяся остовом проводника, не участвует в формировании сил взаимодействия. О непосредственном участии в силовых процессах положительно заряженных ионов говорит хотя бы то, что в процессе сжатия плазмы при протекании через нее постоянного тока происходит сжатие и ионов (так называемый пинч-эффект).

Рассмотрим силовое взаимодействие между двумя параллельными проводниками (рис. 14), по которым текут токи, в рамках концепции скалярновекторного потенциала, определяя этот потенциал одиночного заряда через (16.4) и считая, что его электрические поля действуют на все окружающие заряды, в том числе заряды положительно заряженной решетки.

Пусть $g_{1}^{+}, g_{2}{ }^{+}$и $g_{1}^{-}, g_{2}^{-}$- погонные неподвижные (положительно заряженная решётка в нижнем и верхнем проводниках) и движущиеся заряды, причём оба проводника до начала движения зарядов электронейтральны.

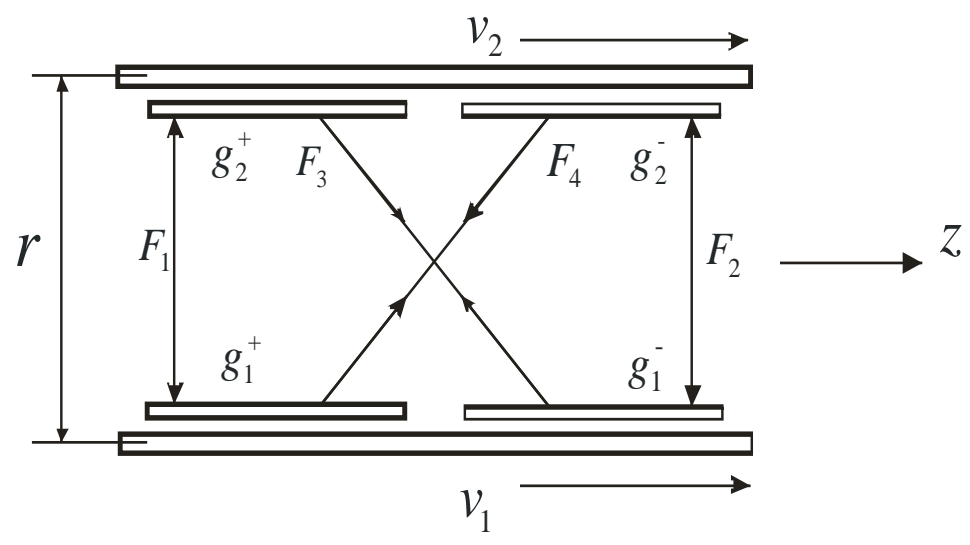

Рис. 14. Схема силового взаимодействия токонесущих проводов

На рис. 14 системы взаимно вложенных разноимённых зарядов для удоб- 
ства рассмотрения раздвинуты по оси $z$. Подсистемы с отрицательным зарядом (электроны) могут двигаться со скоростями $v_{1}$ и $v_{2}$. Сила взаимодействия между проводниками равна сумме сил отталкивания $F_{1}$ и $F_{2}$ (их берём со знаком минус) и сил притяжения $F_{3}$ и $F_{4}$ (со знаком плюс).

Для единичного участка двухпроводной линии силы, действующие между отдельными подсистемами, запишутся в виде:

$$
F_{1}=-\frac{g_{1}^{+} g_{2}^{+}}{2 \pi \varepsilon r}, \quad F_{2}=-\frac{g_{1}^{-} g_{2}^{-}}{2 \pi \varepsilon r} \operatorname{ch} \frac{v_{1}-v_{2}}{c}, \quad F_{3}=\frac{g_{1}^{-} g_{2}^{+}}{2 \pi \varepsilon r} \operatorname{ch} \frac{v_{1}}{c}, \quad F_{4}=\frac{g_{1}^{+} g_{2}^{-}}{2 \pi \varepsilon r} \operatorname{ch} \frac{v_{2}}{c}(19.1)
$$

Суммарная сила, приходящаяся на единицу длины проводника, равна

$$
F_{\Sigma}=\frac{g_{1} g_{2}}{2 \pi \varepsilon r}\left(\operatorname{ch} \frac{v_{1}}{c}+\operatorname{ch} \frac{v_{2}}{c}-\operatorname{ch} \frac{v_{1}-v_{2}}{c}-1\right),
$$

где $g_{1}$ и $g_{2}$ - абсолютные величины зарядов, а знаки сил учтены в выражении в скобках. При $v<<c$ возьмем только два первых члена разложения в ряд $\operatorname{ch}(v / c)$, т.е. будем считать, что $\operatorname{ch}(v / c) \cong 1+v^{2} /\left(2 c^{2}\right)$. Из (19.2) имеем $\left(g_{1}, g_{2}\right.$ - абсолютные величины удельных зарядов, а $v_{1}, v_{2}$ имеют знаки):

$$
F_{\Sigma 1}=g_{1} v_{1} g_{2} v_{2} /\left(2 \pi \varepsilon c^{2} r\right)=I_{1} I_{2} /\left(2 \pi \varepsilon c^{2} r\right)
$$

Поскольку магнитное поле прямого провода с током $I$ равно

$$
H=I /(2 \pi r) \text {, }
$$

то из соотношения (19.3) получаем:

$$
F_{\Sigma 1}=I_{1} I_{2} /\left(2 \pi \varepsilon c^{2} r\right)=H_{1} I_{2} /\left(\varepsilon c^{2}\right)=I_{2} \mu H_{1},
$$

где $H_{1}$ - магнитное поле, создаваемое нижним проводником, по которому течёт ток $I_{1}$, в месте расположения верхнего проводника.

Аналогично,

$$
F_{\Sigma 1}=I_{1} \mu H_{2},
$$

где $H_{2}$ - магнитное поле верхнего проводника в районе нижнего.

Известное правило силового взаимодействия токонесущих систем (19.3) получено не введением феноменологического магнитного поля, а физически прозрачной процедурой в предположении зависимости скалярного потенциала заряда от скорости. В формировании сил взаимодействия в данном случае непосредственно участвует решетка, чего нет в модели магнитного поля. Полученные соотношения совпадают с результатами концепции магнитного поля с постулируемой силой Лоренца, но при этом определены места приложения сил. В данном случае взят только первый член разложения в ряд $\operatorname{ch}(v / c)$. При $v \sim c$ следует брать все члены. Тогда связь между силами взаимодействия и скоростями зарядов нелинейна. Этим предлагаемый метод отличается от метода на основе концепции магнитного поля. Следствие этого - асимметричность закона силового взаимодействия токонесущих систем. При одинаковых значениях, но разных направлениях токов силы отталкивания больше сил притяжения на величину 


$$
\Delta F=\frac{v^{2}}{2 c^{2}} \frac{I_{1} I_{2}}{2 \pi \varepsilon c^{2} \varepsilon},
$$

которая заметна при субсветовых скоростях носителей зарядов.

Если убрать решетку верхнего проводника, оставив поток свободных электронов со скоростью $v_{2}$, то исчезнут силы $F_{1}$ и $F_{3}$. Тогда имеем:

$$
F_{\Sigma}=\frac{g_{1} g_{2}}{2 \pi \varepsilon r}\left(\operatorname{ch} \frac{v_{2}}{c}-\operatorname{ch} \frac{v_{1}-v_{2}}{c}\right) .
$$

Сила Лоренца предполагает линейную зависимость от скорости силы, действующей на заряд, движущийся в магнитном поле. Но в (19.4) зависимость нелинейна, причём с ростом $v_{2}$ отклонение от линейного закона увеличивается, и при $v_{2} \gg v_{1}$ сила стремится к нулю. Это очень важный результат. Этот феномен наблюдали в своих известных экспериментах Томпсон и Кауфман, когда заметили, что с ростом скорости электронного пучка он хуже отклоняется магнитным полем. Результаты своих наблюдений они связали с ростом массы электрона. Как видим, причина здесь другая.

Из (19.3) с точностью до квадратичных членов, сила взаимодействия электронного потока с прямолинейным проводником с током равна:

$$
F_{\Sigma}=\frac{g_{1} g_{2}}{2 \pi \varepsilon r}\left(\frac{v_{1} v_{2}}{c^{2}}-\frac{1}{2} \frac{v_{1}^{2}}{c^{2}}\right) .
$$

Из (19.5) следует, что при однонаправленном движении электронов в проводнике и в электронном потоке взаимодействие при $v_{1}=v_{2} / 2$ отсутствует.

Поскольку скорость электронного потока обычно гораздо выше скорости носителей тока в проводнике, вторым членом в скобках в (19.5) можно пренебречь. Тогда для магнитного поля, создаваемого нижним проводником в месте движения электронного потока, получим:

$$
H_{1}=g_{1} v_{1} /\left(2 \pi \varepsilon c^{2} r\right), F_{\Sigma}=g_{1} g_{2} v_{1} v_{2} /\left(2 \pi \varepsilon r c^{2}\right)=g_{2} \mu v_{2} H
$$

Полученное значение силы совпадает с силой Лоренца. Учитывая, что

$$
F_{\Sigma}=g_{2} E=g_{2} \mu v_{2} H,
$$

можно считать, что на заряд, движущийся в магнитном поле, действует электрическое поле $E$ нормально движению заряда. Данный результат с точностью до квадратичных членов $v^{2} / c^{2}$ определяет силу Лоренца, действующую со стороны магнитного поля на поток движущихся электронов.

Одно из важных противоречий концепции магнитного поля - притяжение двух параллельных пучков одноименных зарядов, двигающихся с одинаковой скоростью в одном направлении. В данной модели этого противоречия уже нет. Если скорости зарядов в верхнем и нижнем проводе равны, а решетка отсутствует (имеются только электронные потоки), то останется только сила отталкивания $F_{2}$ независимо от факта движения зарядов.

Таким образом, движущийся электронный поток взаимодействует одновременно и с движущимися электронами в нижней проволоке, и с её решеткой, а сумма этих сил взаимодействия называется силой Лоренца. Эта сила действует на движущийся поток электронов. Силовое взаимодействие 
между токонесущими системами требует наличия положительно заряженной решетки, вне которой сам движущийся электронный поток не может создавать того же эффекта. Но в двух параллельно движущихся потоках электронов возникает дополнительная сила взаимодействия, зависящая от относительной скорости этих потоков.

Продемонстрируем ещё один подход к проблеме силового взаимодействия токонесущих систем. Наличие сил между токонесущими системами указывает на то, что существует какое-то поле скалярного потенциала, градиент которого обеспечивает указанную силу. Но что это за поле? Соотношение (19.3) даёт только значение силы, но не говорит о том, градиент какого скалярного потенциала эти силы обеспечивает. Этот потенциал есть работа, затраченная при сближении или удалении проводников с неизменными постоянными токами $I_{1}$ и $I_{2}$. Интеграл от (19.3) по $r$ равен энергии:

$$
W=I_{1} I_{2} \ln r /\left(2 \pi \varepsilon c^{2}\right) \text {. }
$$

При удалении проводников она положительна (генератор отдаёт энергию, поддерживая ток в проводниках постоянным), что лежит в основе работы всех электродвигателей. При сближении проводников работу совершают внешние силы над источником, поддерживающим в них постоянство токов, что лежит в основе работы механических генераторов ЭДС.

Соотношение для энергии можно переписать и так:

$$
W=I_{1} I_{2} \ln r /\left(2 \pi \varepsilon c^{2}\right)=I_{2} A_{z 1}=I_{1} A_{z 2},
$$

где $A_{z 1}=I_{1} \ln r /\left(2 \pi \varepsilon c^{2}\right)-z$ - компонента векторного потенциала, создаваемая нижним проводником в месте расположения верхнего проводника, $A_{z 2}=I_{2} \ln r /\left(2 \pi \varepsilon c^{2}\right)-z$ - компонента векторного потенциала, создаваемая верхним проводником в месте расположения нижнего проводника.

Рассмотренный подход демонстрирует большую роль векторного потенциала в силовом взаимодействии токонесущих систем и преобразовании электрической энергии в механическую. Сила Лоренца есть следствие взаимодействия токонесущих систем с полем векторного потенциала, создаваемого другими токонесущими системами. Важно то, что формирование векторного потенциала обязано зависимости скалярного потенциала от скорости, что физически ясно. Движущиеся заряды, в связи с наличием такой зависимости, создают скалярное поле, градиент которого даёт силу. Но создание любого силового поля требует затрат энергии, что совершает генератор, создавая токи в проводниках. В окружающем пространстве создаётся особое поле, взаимодействующее с другими движущимися зарядами по особым векторным правилам, при которых только скалярное произведение вектора скорости заряда и векторного потенциала даёт потенциал, градиент которого есть сила Лоренца, действующая на данный заряд.

Несмотря на простоту и очевидность такого подхода, этот простой механизм до настоящего времени не был окончательно осознан, и сила Лоренца вводилась в классической электродинамике аксиоматическим путём. 
Рассмотрим ещё одно интересное следствие приведенного рассмотрения. Если в качестве плоскостей длинной линии использовать сверхпроводник, то магнитное поле на его поверхности равно удельному току:

$$
H=n e v \lambda,
$$

где $\lambda=\sqrt{m /\left(n e^{2} \mu\right)}$ - глубина проникновения в него магнитного поля.

Подстановка $\lambda$ в (19.6) даёт неожиданный результат - магнитное поле зависит от массы носителей тока, но не величины их зарядов:

$$
H=v \sqrt{n m / \mu} \text {. }
$$

Таким образом, удельная энергия магнитных полей

$$
W_{H}=\mu H^{2} / 2=n m v^{2} / 2
$$

равна удельной кинетической энергии движения зарядов. Но эти поля, связанные с движением носителей тока в поверхностном слое сверхпроводника, занимают несоизмеримо больший объём, чем скинслой. Если обозначить длину линии на рис. 2 через $l$, то объём скинслоя в сверхпроводящих плоскостях линии равен $2 l b \lambda$, энергия магнитных полей в этом объёме -

$$
W_{H, \lambda}=n m v^{2} l b \lambda
$$

энергия же магнитных полей, накопленная между плоскостями линии, -

$$
W_{H, a}=n m v^{2} l b a / 2=l b a \mu_{0} H / 2 .
$$

Так как глубина проникновения магнитного поля в сверхпроводниках составляет несколько сотен ангстрем, то при макроскопических размерах линии можно полную энергию магнитных полей в ней определять (19.8). Поэтому образование магнитных полей $H$ между плоскостями линии в связи с движением зарядов в скин-слое требует таких же затрат энергии, как если бы весь объём линии был заполнен частицами, движущимися со скоростью $v$, плотность и масса которых составляют соответственно $n$ и $m$.

Эффективная масса электрона по сравнению с массой свободного электрона возрастает при этом в $a /(2 \lambda)$ раз. Это следствие того, что механическое движение электронов приводит не только к накоплению их кинетической энергии в скинслое, но и по причине зависимости их скалярного потенциала от скорости в линии происходит также накопление и потенциальной энергии, градиент которой даёт силу, действующую на проводящие плоскости линии. Становится понятной природа индуктивности и эффективной массы электрона, зависящие в данном случае, в основном, не от массы свободных электронов, а от конфигурации проводников.

Униполярная индукция открыта Фарадеем почти 200 лет назад, но нет объяснения того, как и почему работает униполярный генератор. Весьма непонятен случай вращающегося намагниченного проводящего цилиндра, при движении которого между неподвижными контактами, подключёнными к его оси и образующей, возникает ЭДС. Ещё непонятнее, когда вместе с цилиндрическим магнитом вращается проводящий диск, а неподвижные контакты подключены к оси диска и его образующей. В некоторых источниках говорится без какой-либо конкретики, что объяснение даёт СТО. Концепция скалярно-векторного потенциала даёт конкретное объяснение. 


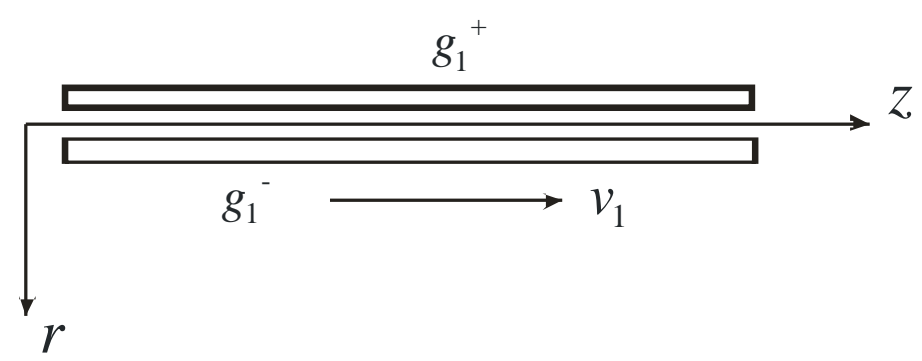

Рис. 15. Проводник, по которому течёт ток

Рассмотрим одиночный длинный проводник с током, по-прежнему считая, что в отсутствие тока взаимно вложенные заряды положительной решетки $g^{+}$и свободных электронов $g^{-}$нейтрализуют друг друга (рис. 15).

Неподвижная решетка создаёт в зависимости от расстояния $r$ от центра расположенного по оси $z$ проводника электрическое поле в направлении $r$

$$
E^{+}=g^{+} /(2 \pi \varepsilon r) .
$$

При скорости $v_{1}$ электронного потока его электрическое поле равно:

$$
E^{-}=-\frac{g^{-}}{2 \pi \varepsilon r} \operatorname{ch} \frac{v_{1}}{c} \cong-\frac{g^{-}}{2 \pi \varepsilon r}\left(1+\frac{1}{2} \frac{v_{1}^{2}}{c^{2}}\right) .
$$

Сумма (19.9) и (19.10) даёт электрическое поле отрицательного заряда

$$
E^{-}=-g^{-} v_{1}^{2} /\left(4 \pi \varepsilon c^{2} r\right)
$$

вокруг проводника с током. Оно очень незначительно, поскольку в реальных проводниках $v \square c$, и его можно обнаружить только при достижимых в сверхпроводниках плотностях токов.

Рассмотрим случай, когда проводник, по которому со скоростью $v_{1}$ текут электроны, движется в обратном направлении со скоростью $v$ (рис. 16).

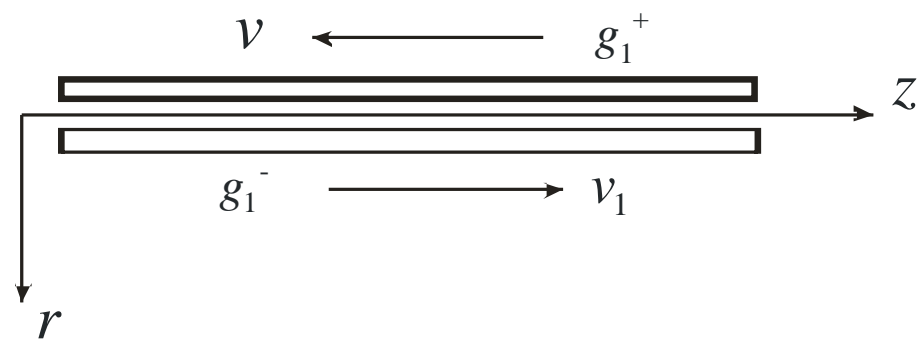

Рис. 16. Движущийся проводник с током

В этом случае соотношения (19.9) и (19.10) примут вид:

$$
\begin{gathered}
E^{+}=\frac{g^{+}}{2 \pi \varepsilon r}\left(1+v^{2} /\left(2 c^{2}\right)\right) ; \\
E^{-}=-\frac{g^{-}}{2 \pi \varepsilon r}\left(1+\left(v_{1}-v\right)^{2} /\left(2 c^{2}\right)\right) .
\end{gathered}
$$

Складывая (19.11) и (19.12), получаем:

$$
E^{+}=\frac{g}{2 \pi \varepsilon r}\left(v_{1} v / c^{2}-v_{1}^{2} /\left(2 c^{2}\right)\right) .
$$


В качестве удельного заряда взята его абсолютная величина. Т.к. скорость механического движения проводника значительно больше дрейфовой скорости электронов, вторым членом в скобках в (19.13) можно пренебречь:

$$
E^{+}=g v_{1} v /\left(2 \pi \varepsilon c^{2} r\right) \text {. }
$$

Вокруг движущегося проводника с током образуется электрическое поле (19.14), что равнозначно появлению удельного положительного заряда

$$
g^{+}=g v_{1} v / c^{2} .
$$

Если проводник свернуть в кольцо и вращать с линейной скоростью $v$, то вокруг такого кольца появится электрическое поле, соответствующее указанному удельному заряду. Так виток (вращающийся магнит) обретает удельный электрический заряд на своей проволоке. При движении линейного проводника с током электрическое поле будет наблюдаться по отношению к неподвижному наблюдателю, но если наблюдатель будет двигаться вместе с проводником, то такие поля будут отсутствовать.

Униполярную индукцию на неподвижных контактах покажем на рис. 17.

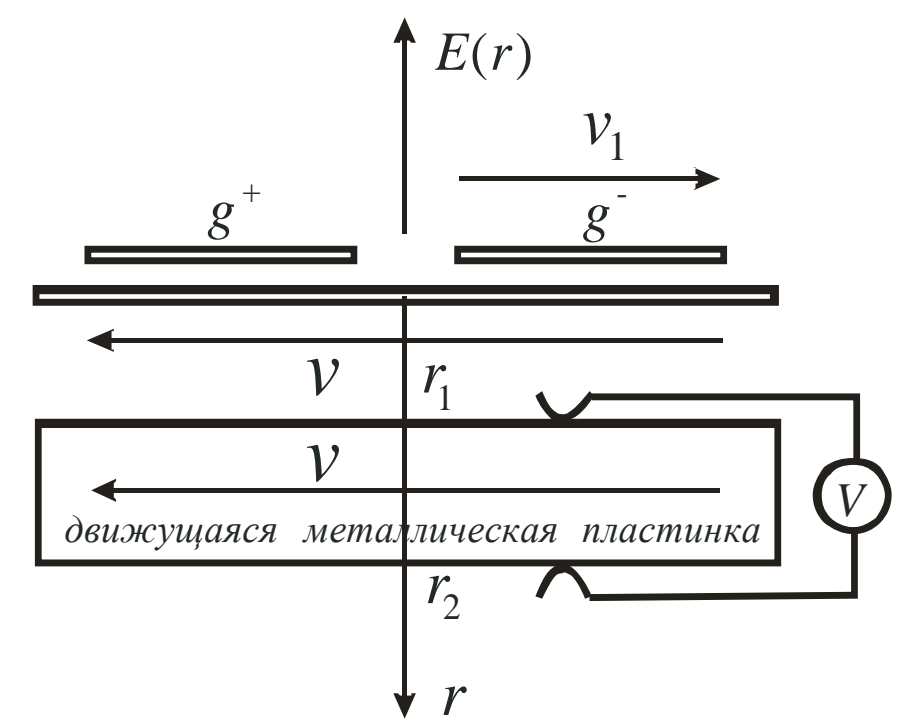

Рис. 17. Схема формирования ЭДС униполярной индукции

Пусть $r_{1}$ и $r_{2}$ - координаты точек касания подвижных контактов, которые скользят по металлической пластине, движущейся с такой же скоростью, что и проводник с током. Контакты подключены к неподвижному вольтметру. Разность потенциалов между ними есть интеграл от (19.14):

$$
U=\frac{g v_{1} v}{2 \pi \varepsilon c^{2}} \int_{r_{1}}^{r_{2}} \frac{d r}{r}=\frac{g v_{1} v}{2 \pi \varepsilon c^{2}} \ln \frac{r_{2}}{r_{1}} .
$$

Чтобы к нагрузке (вольтметру) приложить эту разность потенциалов, нужно скользящие контакты замкнуть перемычкой, на которой нет указанной разности потенциалов. Т.к. пластина движется совместно с проводником, на ней нет разности потенциалов. Она служит той перемычкой и превращает такой составной контур в источник ЭДС по отношению к вольтметру.

Можно получить вращающийся магнит, свернув проволоку в кольцо из одного или нескольких витков (рис. 18) и запитав его от источника тока. 
Причём контакты 1 следует вывести на кольцевые коллекторы, находящиеся на оси вращения, и к ним присоединить трущиеся неподвижные щётки. В этот магнит следует поместить проводящий диск с отверстием, вращающийся совместно с витками магнита, и при помощи неподвижных контактов, скользящим по образующим диска, подать напряжение на вольтметр. В качестве предельного случая можно взять сплошной металлический диск и подключить скользящие контакты к образующей диска и его оси. Вместо вращающегося витка с током можно взять диск, намагниченный в осевом направлении, который эквивалентен витку с током, эффект тот же.

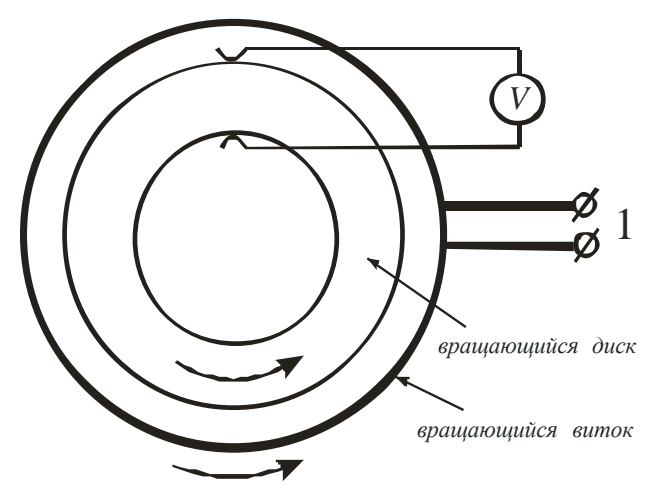

Рис. 18. Схема униполярного генератора с вращающимся магнитом

Среди разных сочетаний вращающихся магнитов и дисков есть случай с неподвижным магнитом и вращающимся проводящим диском (рис. 19).

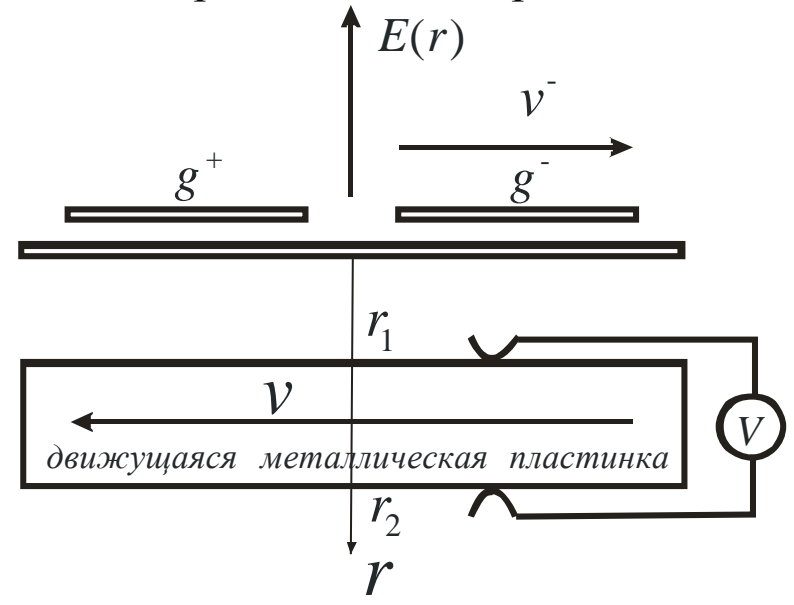

Рис. 19. Случай неподвижного магнита и вращающегося диска

Проводящую пластину нужно свернуть в кольцо. Электрическое поле, генерируемое во вращающемся диске движущимися электронами, равно

$$
E^{-}=-\frac{g^{-}}{2 \pi \varepsilon r} \operatorname{ch} \frac{v_{1}-v}{c}=-\frac{g^{-}}{2 \pi \varepsilon r}\left(1+\left(v_{1}-v\right)^{2} /\left(2 c^{2}\right)\right),
$$

а неподвижными ионами - соотношением

$$
E^{+}=\frac{g^{+}}{2 \pi \varepsilon r} \operatorname{ch} \frac{v}{c}=\frac{g^{-}}{2 \pi \varepsilon r}\left(1+v^{2} /\left(2 c^{2}\right)\right) .
$$

Суммарная напряженность электрического поля при этом составит 


$$
E_{\Sigma}=\frac{g}{2 \pi \varepsilon r}\left(\frac{v v_{1}}{c^{2}}\right),
$$

а разность потенциалов между точками $r_{1}$ и $r_{2}$ в системе координат, движущейся вместе с пластиной, будет равна

$$
U=\frac{g\left(r_{2}-r_{1}\right)}{2 \pi \varepsilon r}\left(\frac{v v_{1}}{c^{2}}\right) \text {. }
$$

Поскольку в цепи вольтметра, неподвижной относительно магнита, нет индуцированной разности потенциалов, указанная разность потенциалов равна ЭДС генератора. Как и ранее, движущуюся проводящую пластинку можно свернуть в диск с отверстием, а проволоку с током - в кольцо (эквивалент магнита, намагниченного в торцевом направлении.

Таким образом, концепция скалярно-векторного потенциала даёт исчерпывающие объяснения, причём без использования СТО.

С точки зрения классической электродинамики предполагается, что давление ЭМ волны связано с наличием у неё механического импульса, и при её падении на поверхность действует закон его сохранения. Но пондеромоторное (механическое) действие на поверхность оказывают не только ЭМ волны, а и постоянные поля, например, магнитное поле, которое оказывает давление на поверхность сверхпроводников. В этом случае объяснить это явление наличием импульса у магнитного поля не представляется возможным. Более того, пондеромоторные силы действуют не только на саму поверхность, но и в толще проводников, где протекают токи.

Само понятие о том, что ЭМ волна оказывает давление на поверхность, не имеет определённого смысла. С точки зрения электродинамики следует рассматривать те последствия, которые имеют место при падении ЭМ волны на границу раздела двух сред, или её проникновение в толщу образца. Такой же подход следует использовать при рассмотрении действия постоянных или переменных полей на границы между средами, где параметры среды меняются плавно или скачкообразно. При падении ЭМ волны на границу раздела в этой области возникают токи, в том числе токи смещения, которые приводят то ли к полному, то ли к частичному отражению волны или изменению её направления. Наличие таких токов, в свою очередь, приводит к возникновению градиентов потенциальной энергии на границе в некоторой области, что и приводит к силовым воздействиям.

С этих позиций можно единообразно рассматривать пондеромоторное действие электрических и магнитных полей на любую границу раздела. Градиент скалярного произведения тока в области границы на векторный потенциал даёт силу, действующую на поверхность. Зависимость градиента потенциальной энергии от координаты даёт информацию о внутренних напряжениях в области границы.

Легче всего это видно на примере сверхпроводников при падении на них ЭМ волн, или наложения постоянных магнитных полей. В сверхпроводниках плотность тока однозначно связана с векторным потенциалом, а произведение тока на векторный потенциал есть потенциальная энергия. Но поскольку токи в сверхпроводнике убывают экспоненциальным образом, то и 
потенциальная энергия этих токов убывает таким же образом, а градиент потенциальной энергии в поверхностном слое и на границе сверхпроводника является причиной появления пондеромоторных сил. Вот каким опосредованным образом магнитное поле и падающая электромагнитная волна оказывает давление на поверхность сверхпроводника.

Плотность тока в сверхпроводнике изменяется по закону

$$
\mathbf{j}(z)=\mathbf{j}(0) e^{-z / \lambda}
$$

По такому же закону изменяется и векторный потенциал А. Поэтому потенциальная энергия токов, текущих в сверхпроводнике, равна

$$
W=\frac{1}{2} \mu \int \mathbf{j} \mathbf{A} d V=\frac{1}{2} \mu \int \mathbf{j}(0) \mathbf{A}(0) e^{-2 z / \lambda} d z .
$$

Следовательно, пондеромоторная сила имеет величину

$$
\mathbf{F}=-\operatorname{grad} W=\frac{1}{2} \mu \mathbf{j}(0) \mathbf{A}(0) e^{-2 z / \lambda} .
$$

Плотность тока в сверхпроводнике определяется соотношением

$$
\mathbf{j}=-\frac{\mu}{L_{k}} \mathbf{A}
$$

где $L_{k}=m /\left(n e^{2}\right)$ - кинетическая индуктивность зарядов, $\lambda=\sqrt{L_{k} / \mu}$ - лондоновская глубина проникновения.

Поэтому имеем:

$$
\mathrm{F}=\frac{1}{2} \mathrm{j}^{2}(0) L_{k} e^{-2 z / \lambda}
$$

На поверхности эта сила равна

$$
\mathrm{F}=\frac{1}{2} \mathrm{j}^{2}(0) L_{k} .
$$

Магнитное поле на поверхности сверхпроводника определяется удельным током, текущим под поверхностью:

$$
H=I=\int j(0) e^{-z / \lambda} d z=\lambda j(0) .
$$

Следовательно, к поверхности сверхпроводника приложена сила

$$
\mathrm{F}=\frac{1}{2} \mathrm{j}^{2}(0) L_{k}=\frac{1}{2} H^{2} \frac{L_{k}}{\lambda^{2}}=\frac{1}{2} \mu H^{2} .
$$

Падающая на поверхность сверхпроводника электромагнитная волна

$$
H=H_{0} \sin \omega t
$$

генерирует на его поверхности удельный ток

$$
I=H=H_{0} \sin \omega t .
$$

Пондеромоторная сила при этом равна

$$
\mathrm{F}=\frac{1}{2} \mu H_{0}^{2} \sin ^{2} \omega t .
$$

Постоянная составляющая такой силы определится соотношением

$$
\mathrm{F}=\frac{1}{4} \mu H_{0}^{2}
$$


Поскольку сверхпроводник не поглощает энергию электромагнитной волны, то она будет полностью отражена. Это равноценно упругому отражению материального объекта от поверхности тела, масса которого значительно больше, чем масса падающего тела.

\section{§ 20. Экспериментальное подтверждение зависимости скалярного потенциала заряда от его относительной скорости}

Положим в (19.1) $g_{2}^{+}=0$ и $v_{2}=0$ (взаимодействие нижнего проводника с током и неподвижного заряда верхнего проводника $g_{2}^{-}$без решетки):

$$
F_{\Sigma 2}=-g_{1} g_{2} v_{1}^{2} /\left(4 \pi \varepsilon c^{2} r\right) .
$$

Значит, когда по проводнику течёт ток, он теряет электронейтральность и обретает вокруг себя радиальное статическое электрическое поле

$$
E_{\perp}=-g_{1} v_{1}^{2} /\left(4 \pi \varepsilon c^{2} r\right),
$$

что эквивалентно появлению на нижнем проводнике дополнительных отрицательного потенциала и удельного статического заряда

$$
g=-2 g_{1} v_{1}^{2} / c^{2} .
$$

Этот факт свидетельствует о том, что принятие концепции скалярновекторного потенциала означает признание зависимости заряда от скорости. Однако до настоящего времени никто не получал экспериментального подтверждения справедливости соотношений (20.1) и (20.2).

В то время, когда Фарадеем и Максвеллом были сформулированы основные законы электродинамики, экспериментально подтвердить соотношение (20.1) было невозможно, т.к. плотности токов, достижимые в обычных проводниках, слишком малы для экспериментального обнаружения рассматриваемого эффекта. Таким образом, положение о независимости скалярного потенциала и заряда от скорости и последующее введение магнитного поля были сделаны волевым путём на феноменологической основе.

Плотности токов, которые могут быть достигнуты в сверхпроводниках, позволяют экспериментально обнаружить электрические поля, определяемые (20.1) [32]. Если такие поля будут обнаружены, то это означает, что скалярный потенциал заряда зависит от его относительной скорости.

Рассмотрим постановку эксперимента, который должен дать ответ на поставленные вопросы. Схема эксперимента изображена на рис. 20.

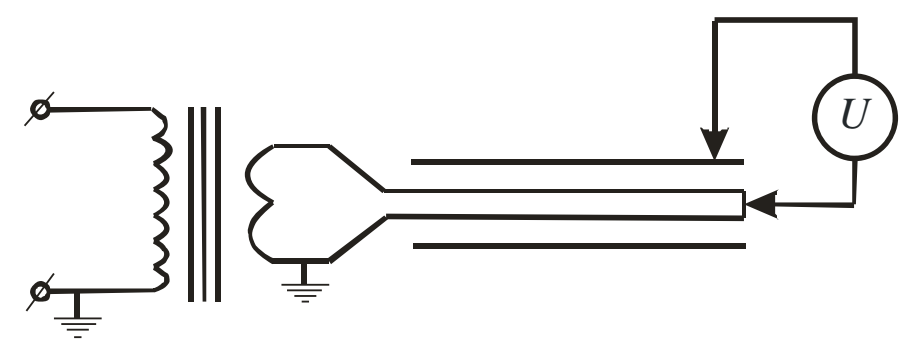

Рис. 20. Схема эксперимента по обнаружению зависимости скалярного потенциала заряда от скорости 
Если сложенную пополам сверхпроводящую проволоку (будем называть ее бифилярной) окружить проводящим цилиндром и ввести в нее ток индукционным способом, то в случае зависимости заряда от скорости электрометр с высоким внутренним сопротивлением, подключенный между цилиндром и проволокой, должен показать наличие разности потенциалов. Бесконтактный индукционный ввод тока применяется с той целью, чтобы исключить наличие контактных разностей потенциалов при контактном вводе тока. Трудность проведения такого эксперимента заключается в том, что входная емкость электрометра (обычно несколько десятков пикофарад) будет значительно больше, чем емкость между бифилярной петлей и цилиндром. Поскольку мы измеряем не ЭДС, а разность потенциалов, обусловленную статическими зарядами, то при подключении к такому устройству входной емкости электрометра заряд, наведенный на цилиндре, перераспределится между обеими емкостями. Если считать, что начальная разность потенциалов между петлей и цилиндром была $U_{1}$, а емкость между ними составляла $C_{1}$, то при подключении между петлей и цилиндром дополнительной емкости электрометра $C_{2}$ разность потенциалов $U_{2}$, которая появится на параллельно включенных емкостях, равна

$$
U_{2}=C_{1} U_{1} /\left(C_{1}+C_{2}\right)=k_{1} U_{1}
$$

Если $C_{1} \square C_{2}$, то $U_{2} \square U_{1}$. Получается, что для получения максимальной разности потенциалов на самом электрометре следует увеличивать емкость между петлей и цилиндром, увеличивая длину всей конструкции.

Расчёт параметров измерительной системы для обнаружения эффекта начнём с определения ожидаемого эффекта. По обе стороны от плоского слоя зарядов с плотностью $n$ и толщиной $\lambda$ создается электрическое поле

$$
E_{\perp}=n e \lambda /\left(2 \varepsilon_{0}\right)
$$

Пока этот слой зарядов не двигается, его электрическое поле полностью скомпенсировано положительными зарядами решетки. Но когда слой начинает двигаться, создается дополнительное электрическое поле, равное

$$
\Delta E \cong E_{\perp} v^{2} /\left(2 c^{2}\right) .
$$

Магнитное поле на поверхности сверхпроводника равно

$$
H=n e v \lambda \text {. }
$$

Подставим в (20.4) найденную из этого соотношения скорость $v$ :

$$
\Delta E_{\perp}=H^{2} /\left(2 \varepsilon_{0} n e \lambda c^{2}\right)=\mu_{0} H^{2} /(2 n e \lambda) .
$$


Для вычисления максимально ожидаемой величины эффекта в качестве $H$ берётся значение критического поля для данного вида сверхпроводника.

Вычислим максимальную величину такого эффекта для сверхпроводящего ниобия, положив: $H_{c}=1,5 \cdot 10^{5} \mathrm{~A} / \mathcal{M}, \lambda \cong 10^{-7} \mathrm{M}, n \cong 3 \cdot 10^{28} \mathcal{M}^{-3}$. Получаем: $\Delta E_{\perp} \cong 3 B / \mu$. Считаем, что диаметр $d$ бифилярной петли равен удвоенному значению диаметра используемой сверхпроводящей проволоки с диаметром 0,25 мм. Если диаметр цилиндра $D=10$ мм, то разность потенциалов между бифилярной петлей и цилиндром $U=\Delta E_{\perp}(d / 2) \ln (D / d) \cong 3 m B$. При этом погонная емкость коаксиала составит $C_{0} \cong 15 n \Phi / \mathrm{M}$.

В экспериментах использовался вибрационный электрометр с входной емкостью $\sim 60$ пФ и чувствительностью $\sim 1 \mathrm{мB}$. Чтобы обеспечить хотя бы такую же емкость коаксиала (при этом разность потенциалов на емкости электрометра после его подключения к коаксиалу составит 1.5 мВ) нужно взять длину коаксиала 4 метра. По техническим причинам такой коаксиал трудно охладить до гелиевых температур, к тому же сам эффект недостаточен для надежного измерения. Поэтому величину эффекта нужно увеличить хотя бы в 100 раз. Это можно сделать, увеличив количество центральных проводов коаксиала до двухсот, для чего понадобится 400 метров проволоки. Конечно, при этом придется увеличить и диаметр цилиндрической его части. Можно опять произвести расчет, но использование экспериментального образца с коаксиалом такого размера все равно неприемлемо ввиду его громоздкости, хотя большим преимуществом такого решения является возможность точного расчета ожидаемого эффекта.

Не столько важно точное совпадение расчетных и экспериментальных данных, сколько надежно обнаружить сам эффект. Поэтому экспериментальный образец создан по другой схеме. Для введения тока в сверхпроводящую низкоиндуктивную обмотку использован охлаждаемый до гелиевых температур трансформатор с железным сердечником. Используя в качестве вторичной обмотки трансформатора сверхпроводящую обмотку, соединенную с низкоиндуктивным соленоидом, можно без наличия гальванических контактов вводить ток в соленоид. В трансформаторе использовался кольцеобразный сердечник из трансформаторной стали с поперечным сечением $9 \mathrm{~cm}^{2}$. Первичная и вторичная обмотки трансформатора были намотаны ниобий-титановым проводом с медным покрытием и содержали 150 и 10 витков соответственно. Таким образом, трансформатор имеет коэффициент трансформации 15. Диаметр проволоки - 0.25 мм. Вторичная обмотка трансформатора соединена последовательно с низкоиндуктивным соленоидом, который намотан бифилярно и содержит 2448 витков такого же провода. Общая длина намотки - 910 м. Концы соленоида и вторичной обмотки трансформатора сварены лазерной сваркой. Соленоид намотан на каркасе из фторопласта. Внутренний и внешний диаметр обмотки соленоида - 35 и 90 мм соответственно, ширина намотки - 30 мм. К средней точке соленоида подключен внутренний провод коаксиала, вы- 
ходящего наружу криостата, такой же коаксиал подключен и к экрану соленоида. Конструкция низкоиндуктивного соленоида показана на рис. 21.

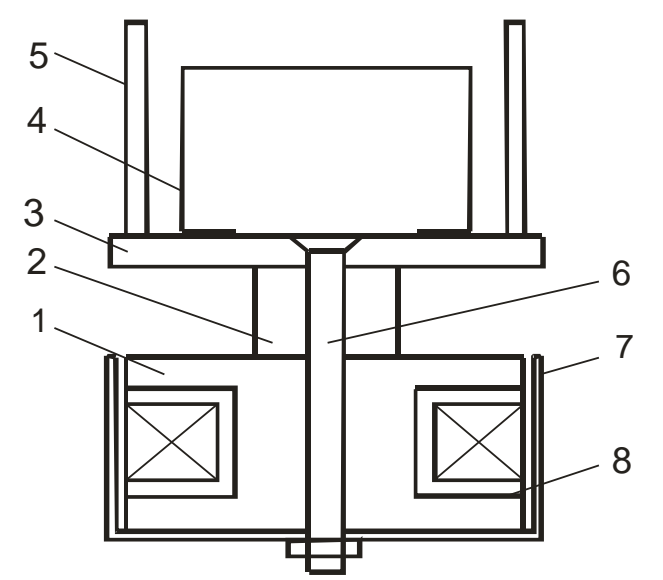

Рис. 21. Конструкция низкоиндуктивного сверхпроводящего соленоида

Цифрами на рис. обозначены: 1 - алюминиевый каркас, 2 - фторопластовая втулка, 3 - фторопластовый диск, 4 - скоба, 5 - стойки, 6 - болт, 7 - медный экран, 8 - фторопластовый каркас. Соленоид намотан на фторопластовом каркасе 8 , который заключён в алюминиевый каркас 1 . Снаружи соленоид окружён медным экраном 7, который совместно с каркасом 1 является экраном соленоида. К каркасу 1 посредством болта 6 и фторопластовой втулки 2 крепится фторопластовый диск 3 , на котором смонтирована скоба 4. Витки вторичной обмотки трансформатора охватывают скобу 4, через которую, не касаясь её, проходит магнитопровод трансформатора. Вся конструкция крепится к трансформатору посредством стоек 5. Трансформатор совместно с соленоидом размещается в баке гелиевого криостата.

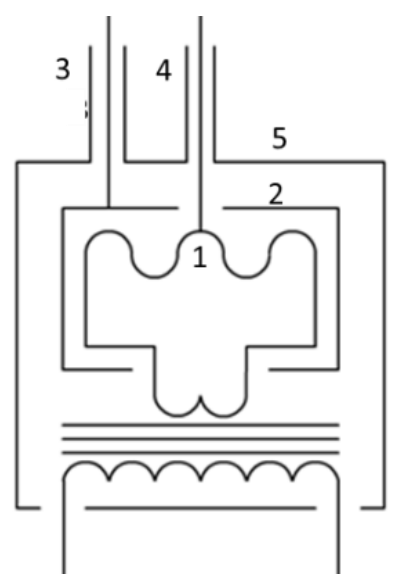

Рис. 22. Схема соединения низкоиндуктивного соленоида

Схема подключения коаксиалов к малоиндуктивному соленоиду показана на рис. 22.

На рис. обозначены: 1 - малоиндуктивный соленоид, 2 - экран соленоида, 3,4 - коаксиалы, 5 - общий экран, которым является гелиевый бак. Сопротивление между заземленными элементами, экраном соленоида и самим соленоидом составляет не менее $10^{14}$ Ом. Ёмкости относительно земли элементов конструкции: коаксиал $3-44$ пФ, коаксиал $4-27$ пФ, емкость 
экран-земля составляет 34 пФ, емкость экран-соленоид составляет 45 пФ. В качестве электрометра использовался емкостной вибрационный электрометр с входной емкостью 60 пФ и входным сопротивлением $10^{14}$ Ом.

Такая конструкция сверхпроводящего соленоида и окружающего экрана позволяет установить наличие самого эффекта без точного электродинамического расчета электростатических полей вокруг соленоида.

При измерениях электрометр подключался непосредственно к экрану посредством коаксиала 4, а средняя точка сверхпроводящего соленоида посредством коаксиала 3 заземлялась. Ток в первичную обмотку трансформатора вводился от источника постоянного тока, показания электрометра при этом не зависели от направления тока. При величинах вводимого тока 9 А происходил самопроизвольный сброс показаний электрометра. Это означает, что ток в обмотке соленоида достигал критического значения, и обмотка переходила в нормальное состояние. Железный сердечник при этом захватывал магнитный поток и при уменышении вводимого в соленоид тока кривая зависимости измеряемого потенциала от тока повторялась, а потенциал достигал максимального значения при нулевом значении тока.

Зависимость измеряемой разности потенциалов приведена на рис. 23.

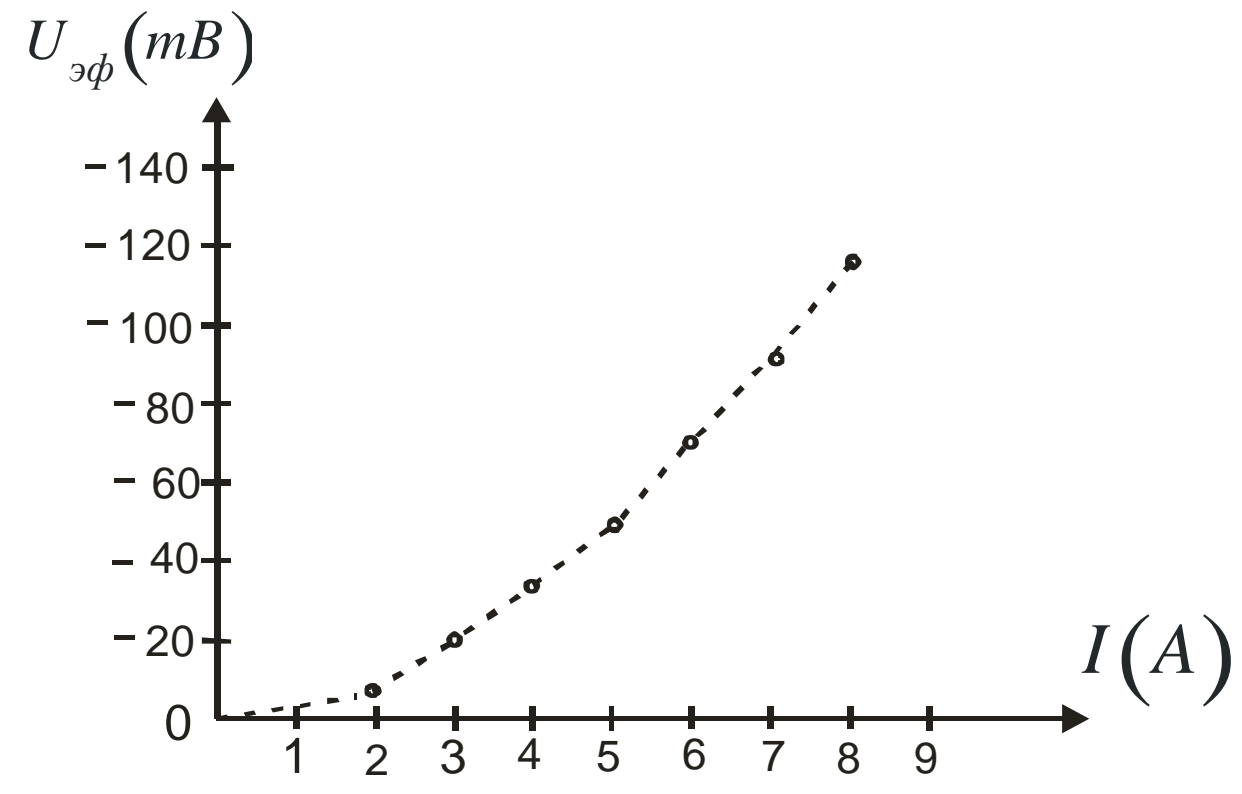

Рис. 23. Зависимость приведенной разности потенциалов между экраном и низкоиндуктивным соленоидом от тока в его обмотке

Экспериментальные данные приведены в следующей таблице.

\begin{tabular}{|l|l|l|l|l|l|l|l|l|}
\hline$I(A)$ & 1 & 2 & 3 & 4 & 5 & 6 & 7 & 8 \\
\hline$I_{1}(A)$ & 15 & 30 & 45 & 60 & 75 & 90 & 105 & 120 \\
\hline$H(A / M) \cdot 10^{4}$ & 1.91 & 3.82 & 5.73 & 7.64 & 9.55 & 11.5 & 14.6 & 15.3 \\
\hline$-U_{2}(M B)$ & - & 2 & 6 & 10 & 15 & 21 & 27 & 35 \\
\hline$-U_{1}(M B)$ & - & 7 & 20 & 34 & 50 & 71 & 90 & 117 \\
\hline$U_{э \phi} / I^{2}(M B / A)$ & - & 1.75 & 2.22 & 2.13 & 2.00 & 1.94 & 1.84 & 1.83 \\
\hline
\end{tabular}


В первой строке таблицы приведены значения вводимого тока $I$. Во второй - значения тока $I_{1}$ в обмотке соленоида с учётом значения 15 коэффициента трансформации. Предполагается, что во всем диапазоне вводимых токов намагниченность сердечника остается пропорциональной току. В третьей - значения магнитных полей на поверхности сверхпроводящих проводов соленоида. В четвертой - показания электрометра. В пятой - эффективные значения разности потенциалов, которые были бы между соленоидом и экраном до подключения к последнему суммарной емкости коаксиала и электрометра. В шестой - коэффициент $k=U_{\text {эф }} / I^{2}$, указывающий на отклонение полученной зависимости от квадратичного закона. Коэффициент $k_{1}$ составил величину 3.35 , он вычислялся по формуле (20.3), исходя из того, что емкость между экраном и соленоидом $C_{1}=45$ пФ, а суммарная емкость $C_{2}$, подключаемая к емкости $C_{1}$ и состоящая из емкостей коаксиала и электрометра, равна 111 пФ. Среднеквадратичное относительное отклонение коэффициента $k$ от своего среднего значения 1.93 составляет 0.13 , что дает относительную среднеквадратичную погрешность $7 \%$. Таким образом, полученная зависимость между током и измеряемым значением потенциала очень близка к квадратичной. Из таблицы видно, что при значениях тока в проводниках соленоида порядка 120 А напряженность поля на их поверхности достигает своего критического значения, которое для используемого сверхпроводника составляет $1.5 \cdot 10^{5} \mathrm{~A} / \mathrm{M}$, с чем и связан сброс показаний электрометра при достижении этих токов. Таким образом, экспериментальные результаты указывают на то, что величина скалярного потенциала, а, следовательно, и заряда зависит от скорости.

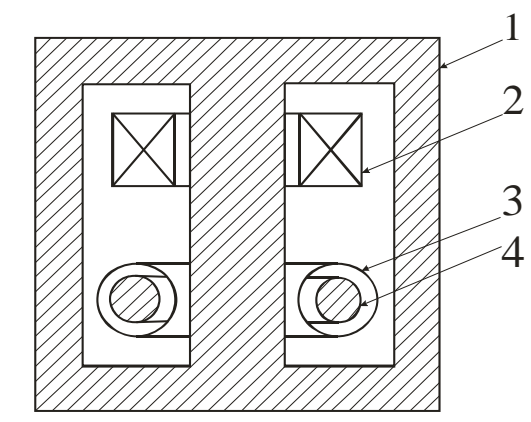

Рис. 24. Схема эксперимента со сверхпроводящим тором

Однако в данной схеме эксперимента имеет место непосредственное гальваническое подключение электрометра к сверхпроводящему соленоиду. Не возникает ли разность потенциалов между соленоидом и экраном по причине каких-то контактных явлений в месте контакта провода, соединяющего электрометр с соленоидом? Для ответа на этот вопрос проведены эксперименты со сверхпроводящим ниобиевым тором (рис. 24).

Если внутри проводящего экрана разместить второй проводящий экран и между ними подключить электрометр, то при появлении заряда внутри внутреннего экрана появится разность потенциалов между экранами. В ка- 
честве наружного экрана 1 использовалось ярмо трансформатора из трансформаторной стали. На центральном стержне этого ярма располагалась первичная обмотка 2, намотанная ниобий-титановым проводом, содержащая 1860 витков. На этом же стержне располагался торообразный металлический экран 3, выполненный из меди. Внутри этого экрана был расположен тор 4, выполненный из ниобия. Наружный диаметр ниобиевого тора составлял 76 мм, а внутренний 49 мм. Трансформатор размещался в баке гелиевого криостата и охлаждался до гелиевой температуры, при этом ярмо трансформатора и гелиевый бак заземлялись.

При введении постоянного тока в первичную обмотку в сверхпроводящем торе индуцировался ток, и электрометр фиксировал разность потенциалов $U$ между экраном 3 и ярмом трансформатора, т.е. ниобиевый тор внутри экрана 3 при введении в него постоянного тока теряет электронейтральность. Величина постоянного тока в сверхпроводящем торе в 1860 раз превышала ток, вводимый в первичную обмотку. Зависимость $U$ от тока $I$, вводимого в первичную обмотку, показана на рис. 25.

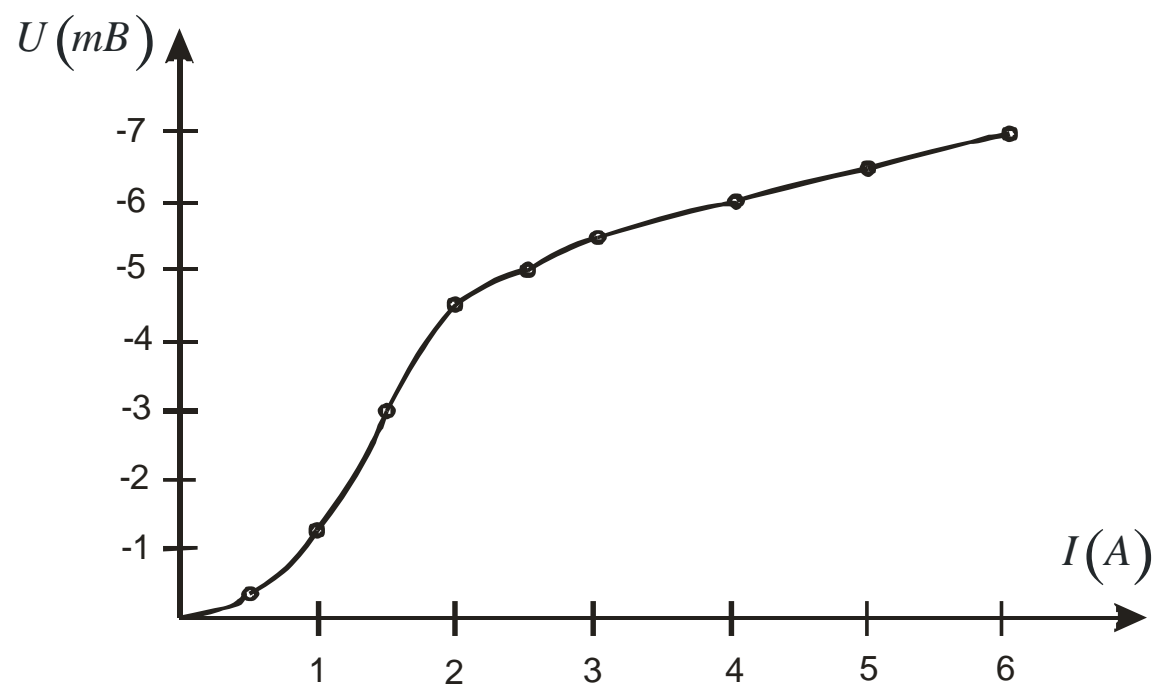

Рис. 25. Зависимость разности потенциалов между экраном 3 и ярмом трансформатора 1 от вводимого в его первичную обмотку тока

Данные значения разности потенциалов значительно меньше, чем для сверхпроводящей проволочной обмотки, т.к. поверхность тора значительно меньше, чем проволочной обмотки. Вид зависимости разности потенциалов от вводимого тока также сильно отличается. Квадратичный участок наблюдается только на очень небольшом начальном участке вплоть до значений токов около 2 ампер, вводимых в первичную обмотку. Далее эта зависимость прямолинейна с малым наклоном к оси абсцисс. К тому же срыва показаний электрометра в данном случае не наблюдалось.

С чем связаны такие отличия? В случае проволочного соленоида сверхпроводящий ток равномерно распределён по поверхности проволоки и достигает критического значения на всех участках одновременно, с чем и связан одновременный переход всей обмотки соленоида в нормальное состояние при достижении на каком-то участке критического значения тока. 
В случае тора процесс установления сверхпроводящего тока на его поверхности происходит по-другому. Вводимый в сверхпроводящий тор постоянный ток очень неравномерно распределён по его поверхности. Плотность тока максимальна на внутренней поверхности тора и значительно меньше на периферии. С этим связано то, что внутренние поверхности тора начинают переходить в нормальное состояние раньше наружных. Процесс перехода тора в нормальное состояние происходит так, что при увеличении тока в торе в нормальное состояние переходят сначала внутренние области, и нормальная фаза начинает перемещаться от внутренних областей к наружным. Процесс длится до тех пор, пока весь тор не перейдёт в нормальное состояние. Но почему при этом к моменту перехода тора в нормальное состояние не происходит сброс тока, как в случае проволочного соленоида? Это связано с тем, что ниобий является сверхпроводником второго рода. Он не сразу скачком переходит в нормальное состояние, а имеет достаточно значительную область плотностей токов, при которых он находится в смешанном состоянии, когда внутрь массивного проводника проникают вихри Абрикосова. Следовательно, отсутствие сброса показаний электрометра указывает на то, что сверхпроводящий тор находится в смешанном состоянии, а вихревые структуры, которые также представляют сверхпроводящие токи, приводят к потере тором электронейтральности. Можно сделать вывод, что вихри несут на себе не только кванты магнитного потока, но ещё и квантованные электрические заряды.

При другом направлении тока в первичной обмотке повторяется зависимость, подобная рис. 25, но с сильным гистерезисом. К нему приводит то, что вихри, проникшие вглубь сверхпроводника, закрепляются на дефектах кристаллической структуры, попадая в потенциальные ямы.

Таким образом, результаты экспериментов однозначно указывают на зависимость скалярного потенциала и величины зарядов от их скорости, что было предсказано ещё в работе [10] и экспериментально подтверждено в работах $[59,60]$. Указанные эксперименты проведены в начале $90-\mathrm{x}$ годов в МГП НИИ криогенного приборостроения ФТИНТ НАН Украины.

Зависимость скалярного потенциала заряда и его величины от скорости исследовались и при разогреве плазмы [50]. В этих экспериментах использовались микровзрывы при разряде электролитических конденсаторов большой ёмкости через разрядник (рис. 26, 27). В разряднике использовалась медная проволока, при подключении к которой заряженных конденсаторов она плавилась и испарялась, превращаясь в плазму.

В клетке Фарадея, которой служил сплошной металлический экран (на рисунках изображена пунктиром) размещались электролитические конденсаторы большой ёмкости, разрядник и ключ, позволяющий подключать к разряднику заряженные конденсаторы. Цепи контура, включающие конденсатор, ключ и разрядник, не имели гальванического контакта с экраном клетки Фарадея. Клетку Фарадея окружает один (рис. 26) или два (рис. 27) 


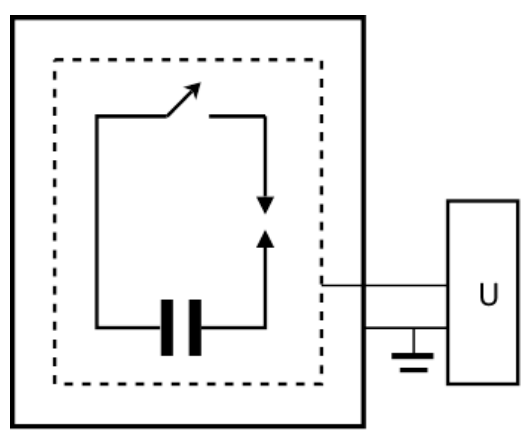

Рис. 26. Схема эксперимента с одним внешним экраном

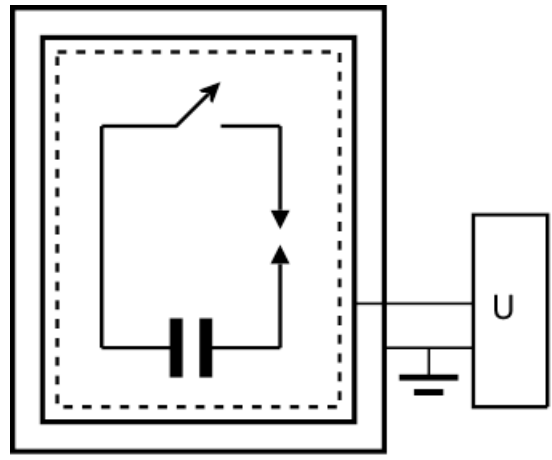

Рис. 27. Схема эксперимента с промежуточным экраном

металлических экрана. Измерение характеристик электрического импульса, возникающего при микровзрыве, осуществлялось при помощи цифрового запоминающего осциллографа SIGLENT SDS 1072CNL.

В первом случае (рис. 26) осциллограф подключался между экраном клетки Фарадея и внешним экраном. Во втором (рис. 27) - между внешним экраном и промежуточным, расположенным между экраном клетки Фарадея и внешним. Функциональная схема установки показана на рис. 28. Составной шток состоит из двух частей. К верхней текстолитовой крепится нижняя латунная крепёжными шпильками. Пружина между нижней частью штока и латунной пластиной обеспечивает электрический контакт между латунной частью штока и латунной пластиной. К перегородке внутри экрана клетки Фарадея крепится изолирующая пластина с контактной шайбой на ней. Блок конденсаторов подключён между латунной пластиной и контактной шайбой. К нижней части штока крепится медная проволока диаметром 0.2 мм, выступая из штока на 10 мм. При опускании штока проволока касается контактной шайбы, к ней подключаются заряженные конденсаторы, проволока плавится и испаряется, превращаясь в плазму. Набор электролитических конденсаторов общей ёмкостью 6000 мкФ заряжался до напряжения 300 В. Крепёжные болты и шпильки показаны жирными отрезками линий. Не показаны разъёмы для подключения осциллографа между экраном клетки Фарадея и внешним экраном, между внешним и промежуточным экраном и разъёмы для зарядки конденсаторов. При измерениях кабель для зарядки конденсаторов от клетки Фарадея отключался. 


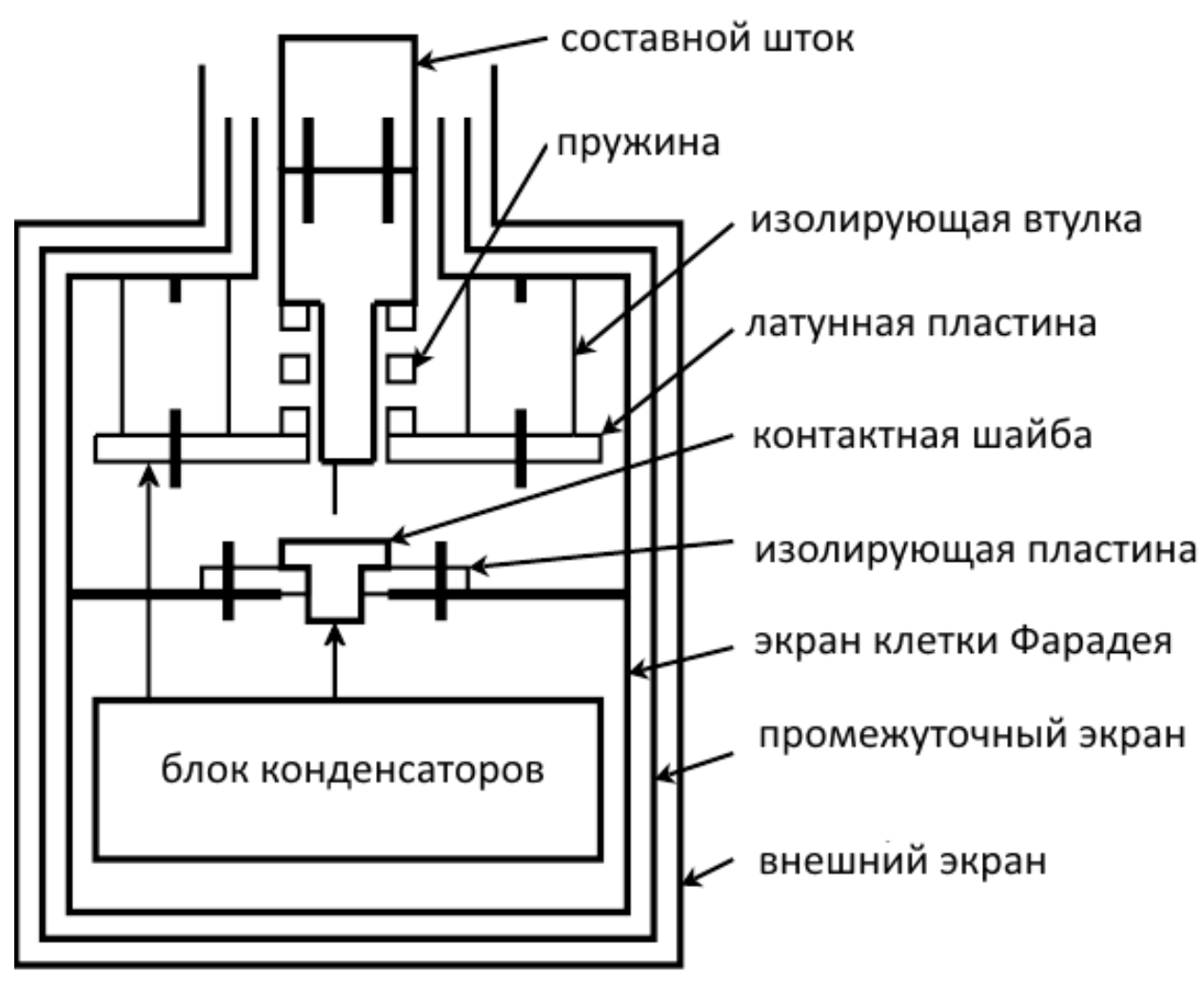

Рис. 28. Функциональная схема экспериментальной установки
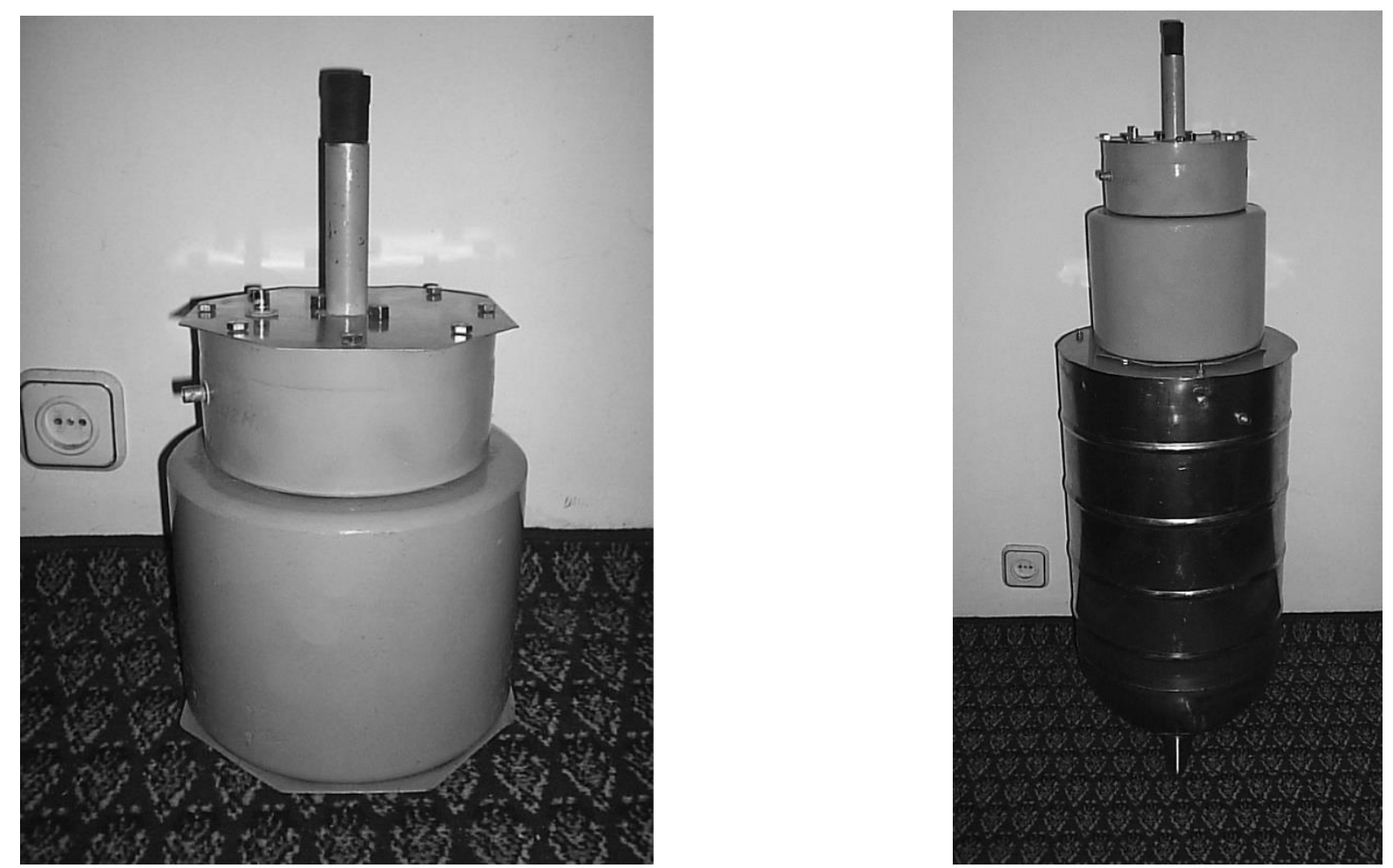

Рис. 29. Фотография клетки Фарадея в сборе
Рис. 30. Фотография экспериментальной установка в разобранном виде 
Фотография клетки Фарадея в сборе показана на рис. 29. Диаметр верхней и нижней части экрана клетки Фарадея 180 мм и 220 мм. Высота верхней части 80 мм, нижней 220 мм. Верхняя часть закрыта крышкой, к которой крепится трубка длиной 100 мм, в которую вставляется составной шток. Экран клетки Фарадея покрыт тремя слоями изолятора - акриловой автоэмали. Поверх может наклеиваться медная фольга - промежуточный экран. На рис. 30 изображены отдельные детали экспериментальной установки. Нижняя часть фотографии представляет внешний экран. Его диаметр 300 мм, а высота 600 мм. Сверху на внешнем экране, закрытом крышкой, стоит клетка Фарадея. В собранном виде установки клетка Фарадея располагается внутри промежуточного или внешнего экрана.

Экспериментально установлено, что при разряде конденсаторов через разрядник между экраном клетки Фарадея и внешним или между промежуточным и внешним экранами возникает импульсное напряжение. Чтобы удостовериться в том, что при разогреве плазмы в клетке Фарадея действительно возникает унитарный отрицательный заряд, проведен следующий эксперимент. После натирания шерстью образца из янтаря (при этом на нём образуется отрицательный заряд), он через трубку в верхней крышке быстро вводился в клетку Фарадея. На осциллографе, подключённом между экраном клетки Фарадея и внешним, зарегистрирован импульс (рис. 31). Форма импульса при быстром изъятии образца заряженного янтаря из клетки Фарадея показана на рис. 32. Если заряженный образец из янтаря быстро ввести в клетку и сразу столь же быстро изъять оттуда, то наблюдается импульс, показанный на рис. 33. Между отрицательной и положительной частями импульса есть область уменьшения производной амплитуды импульса по времени, т.к. при введении и изъятии образца янтаря из клетки Фарадея нельзя мгновенно поменять скорость штока, на которой закреплён образец, на обратную.

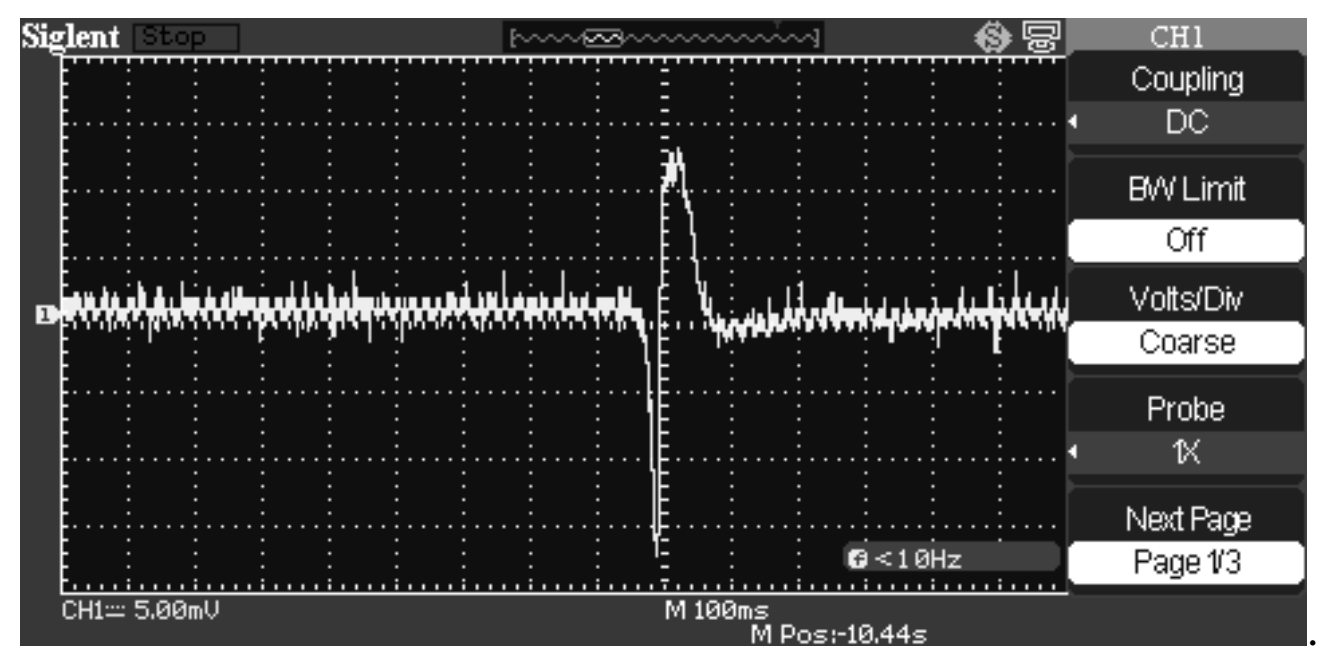

Рис. 31. Форма импульса при быстром введении образца янтаря 


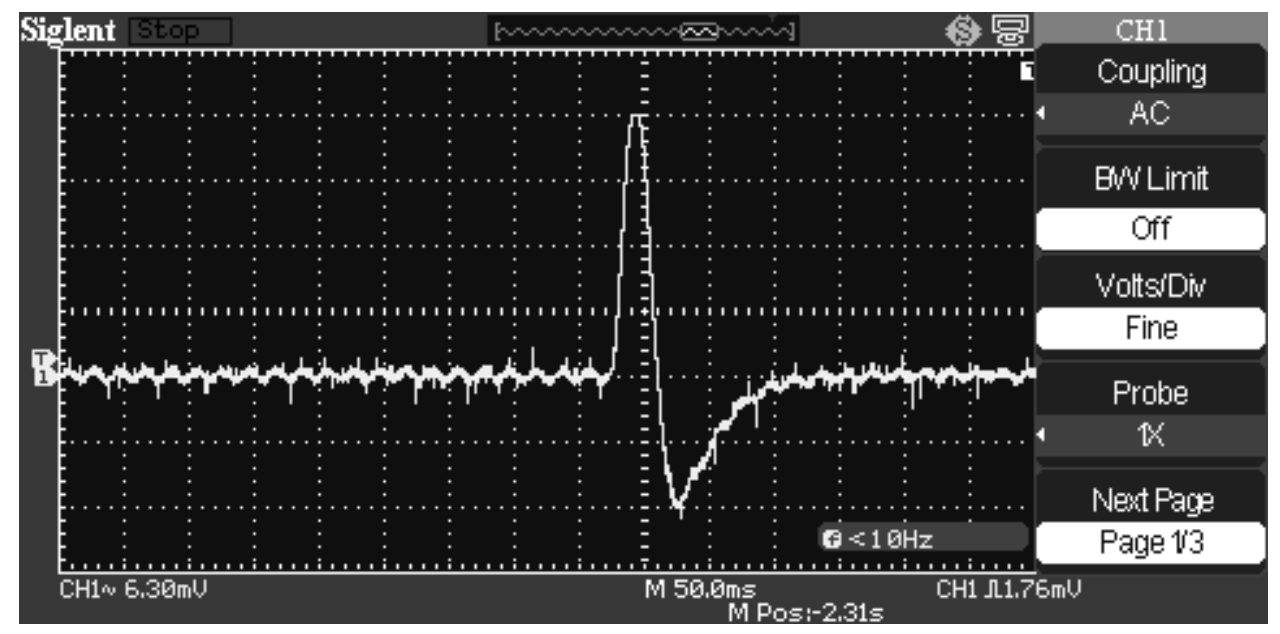

Рис. 32. Форма импульса при быстром изъятии образца янтаря

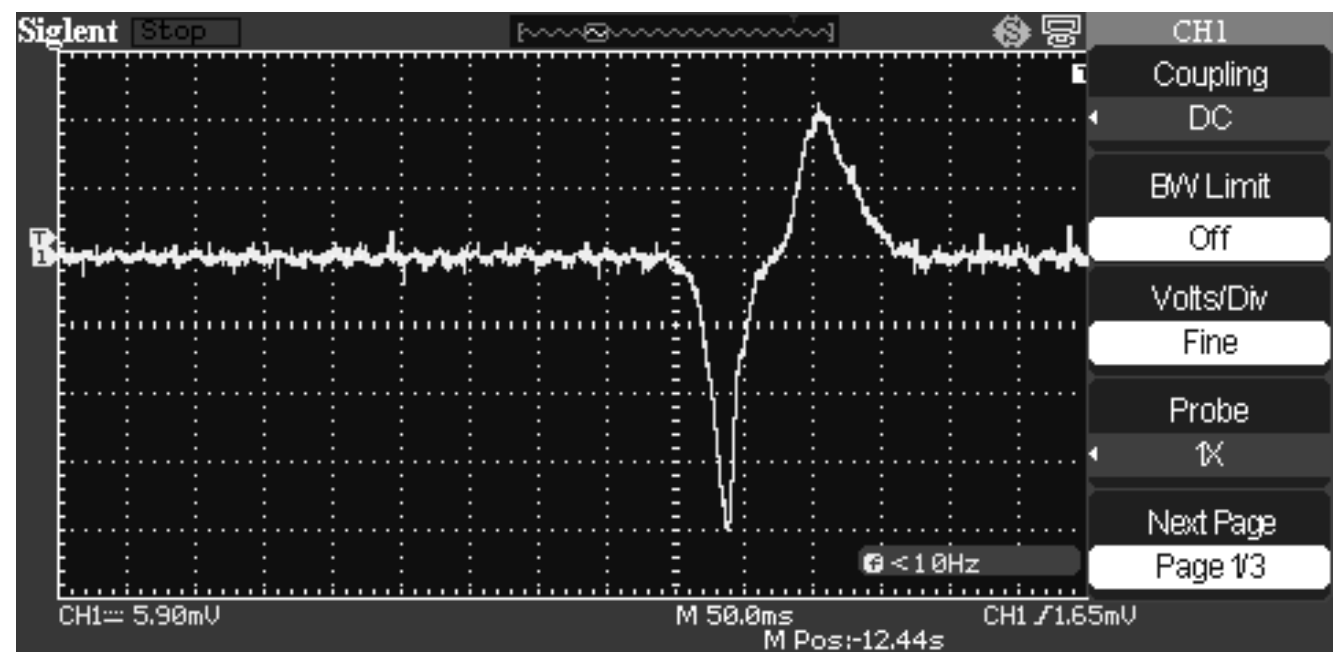

Рис. 33. Форма импульса, полученная при быстром введении и последующем быстром изъятии из клетки Фарадея заряженного образца янтаря

На рис. 34 представлена осциллограмма переходного процесса при разряде конденсаторов через разрядник. За время разряда около 600 мкс напряжение на конденсаторах падает с 300 В до 50 В, а энергия конденсаторов - на 162 Дж, поэтому средняя мощность микровзрыва 270 кВт. Форма импульса напряжения между внешним экраном и экраном клетки Фарадея при разряде показаны на рис. 35 (цена деления по оси X 2.5 мс) и рис. 36 (цена деления 1 мс). Формирование отрицательной части импульса (рис. 36) примерно совпадает с временем разряда конденсаторов (рис. 34). Это время наибольшего разогрева плазмы, т.к. при большом токе разогрев связан не только с её активным сопротивлением, но и с пинч-эффектом. Формы импульсов на рис. 31 и рис. 35 очень похожи. Разница лишь в том, что при механическом введении янтаря в клетку нельзя обеспечить такое время импульса и крутизну его фронтов, как при электрическом разряде. На рис. 35 хорошо видны этапы разогрева и остывания плазмы, видно также, что её нагрев происходит гораздо скорее, чем остывание. 


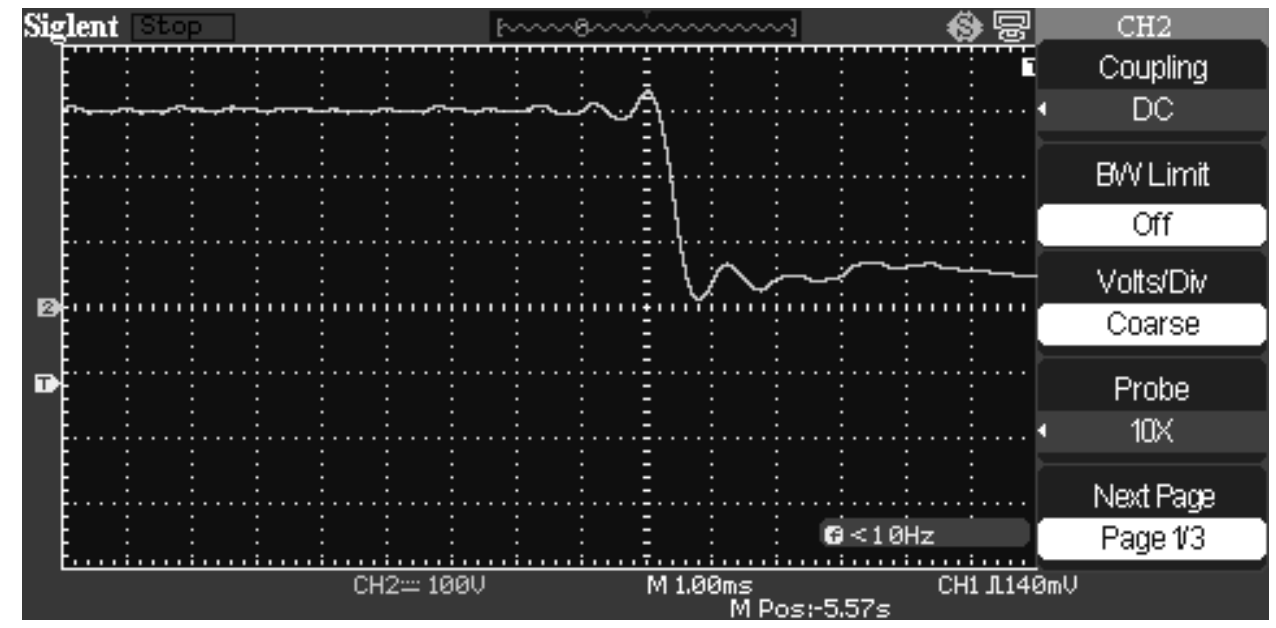

Рис. 34. Осциллограмма переходного процесса

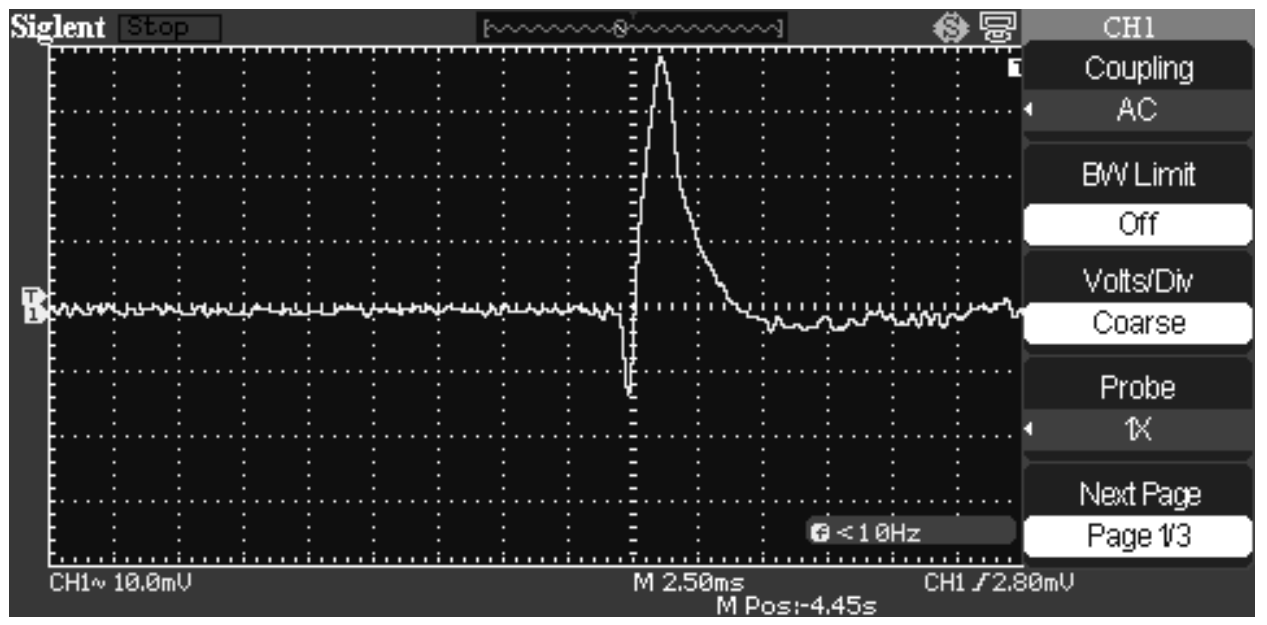

Рис. 35. Форма импульса напряжения

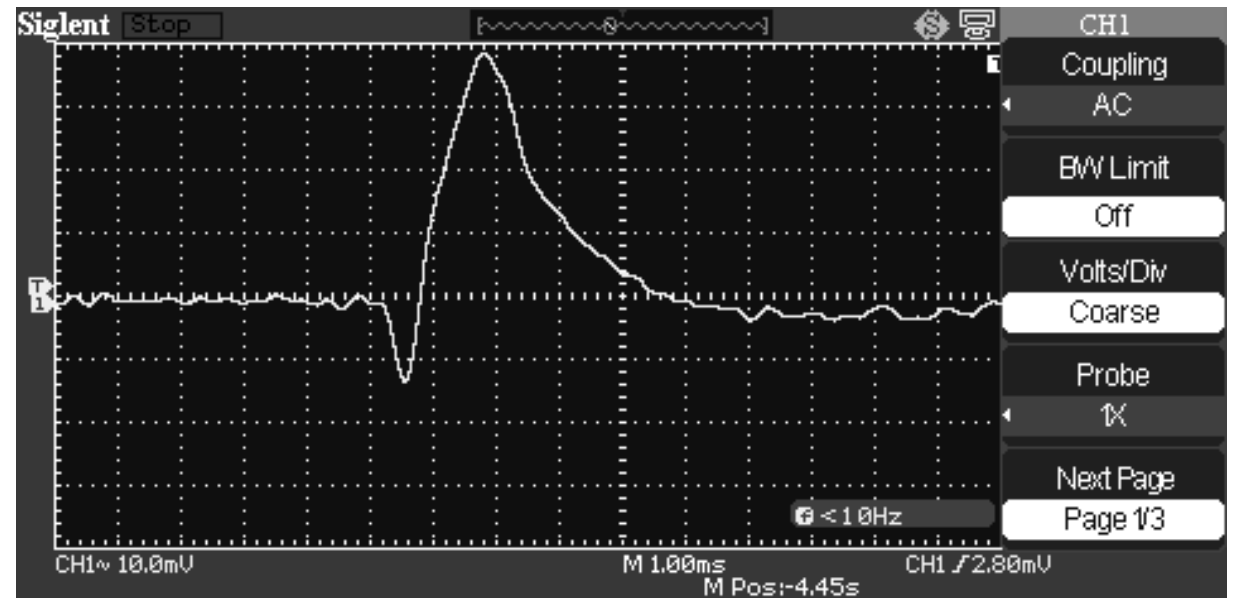

Рис. 36. Форма импульса напряжения

Суммарная ёмкость входной цепи осциллографа и ёмкости между экраном клетки Фарадея и внешним экраном составляет 204 пФ, а сопротивле- 
ние входной цепи осциллографа равно 1Мом, следовательно, входная цепь осциллографа является дифференцирующей.

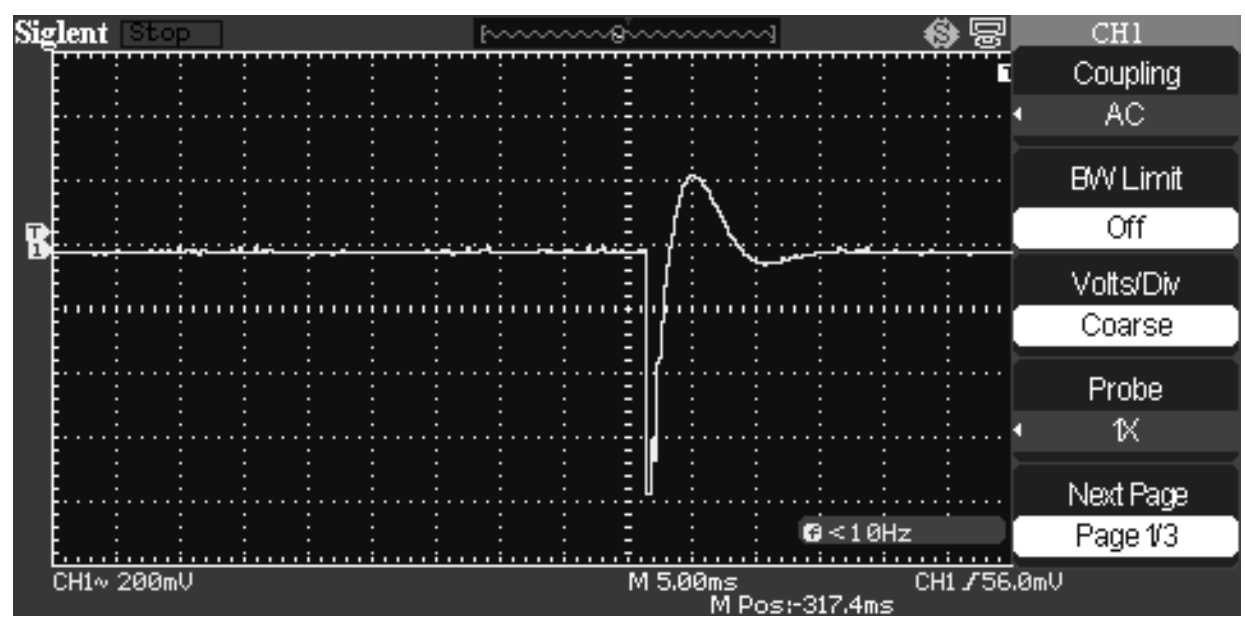

Рис. 37. Производная тока, текущего через плазму

Поэтому осциллограммы на рис. 35 и рис. 36 представляют производную импульса напряжения между экраном клетки Фарадея и внешним. Естественно предположить, что температура плазмы, поскольку она имеет активное сопротивление, пропорциональна току через неё. Производная тока через плазму снималась при помощи цепи, индуктивно связанной с проводниками контура, по которым течёт ток разряда (рис. 37). Импульсы на рис. 35 и рис. 37 идентичны. Это означает рождение и исчезновение в клетке Фарадея унитарного заряда, связанного с движением электронов. В образовавшейся плазме число электронов и положительных ионов одинаково, но электроны быстрее ионов.

Приведенные экспериментальные данные доказывают, что в процессе разогрева плазмы, при равном количестве в ней электронов и положительных ионов, в ней образуется унитарный отрицательный заряд, не скомпенсированный положительными ионами. Эксперимент прямо подтверждает то, что инвариантом скорости является лишь полярность движущегося электрического заряда, а его абсолютная величина зависит от скорости.

\section{§ 21. Электрический импульс ядерного взрыва}

По программе «Starfish» 9 июля 1962 США взорвали в космосе над Тихим океаном водородную бомбу мощностью 1.4 Мт [20, 21]. В 1957 г. будущий нобелевский лауреат доктор Ханс Альбрехт Бете (Hans A. Bethe) на основе теории дипольного излучения предсказал напряженность поля на поверхности земли от электромагнитного импульса (ЭМИ) при подобном взрыве не более 100 В/м. Поэтому вся измерительная аппаратура была настроена на регистрацию таких напряженностей. Но оказалось, что на протяжении более 1000 км от эпицентра взрыва напряженность электрических полей достигла нескольких десятков тысяч вольт на метр. Имеется запись формы ЭМИ на расстоянии 1300 км от места взрыва (рис. 38). 


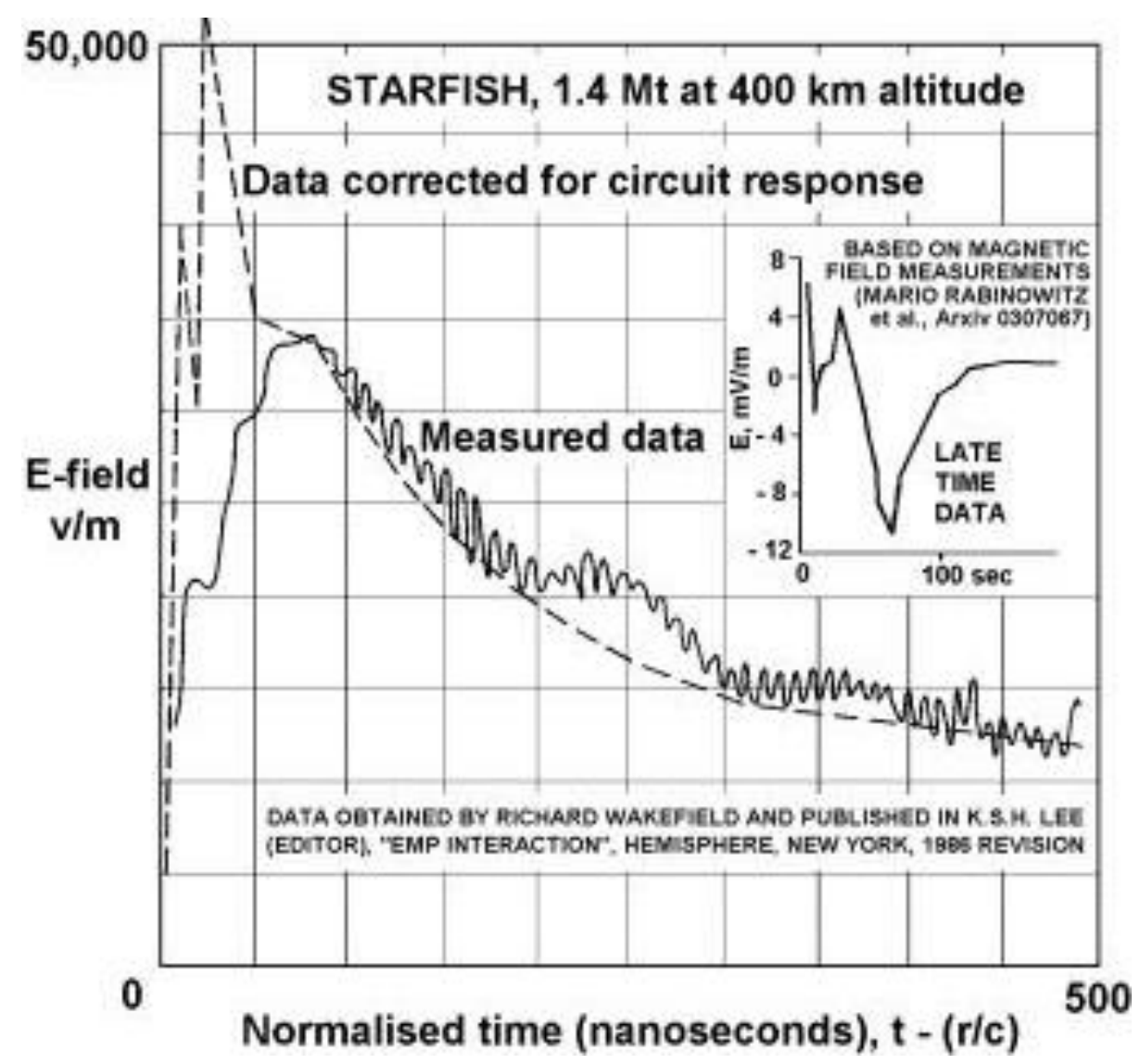

Рис. 38. Экспериментальная зависимость амплитуды ЭМИ от времени

Импульс имеет не только очень большую амплитуду, но и очень малую длительность порядка 150 нс. Поскольку прогноз не оправдался, было выдвинуто ряд теорий, призванных объяснить экспериментальные данные. Наибольшую известность получила теория, в которой предполагается, что формирование импульса обязано релятивистским комптоновским электронам, которые выбивает из молекул воздуха жесткое рентгеновское излучение и которые синфазно с гамма-излучением двигаются с релятивистскими скоростями в направлении распространения электромагнитной волны.

Из этой модели следует, что импульс распространяется из ионосферы в нижние слои атмосферы, и, следовательно, его поля выше ионосферы, непосредственно в самом космосе, отсутствуют. При этом как-то можно объяснить наличие электрических полей в зоне видимости взрыва, но сильное возмущение ионосферы на больших расстояниях от взрыва объяснить трудно. В течение нескольких десятков минут отсутствовала радиосвязь с Японией и Австралией. Даже на расстоянии 3200 км от эпицентра возмущения ионосферы в несколько раз превысили обусловленные самыми мощными вспышками на Солнце. Импульс сразу вывел из строя три космических спутника. В результате захвата магнитосферой Земли заряженных частиц от взрыва их концентрация в искусственном радиационном поясе Земли увеличилась на 2-3 порядка. Воздействие радиационного пояса привело к очень быстрой деградации солнечных батарей и электроники еще у семи спутников, в том числе у первого коммерческого телекоммуни- 
кационного спутника Телестар. Всего вышла из строя треть космических аппаратов, находившихся в момент взрыва на низких орбитах.

При ядерном взрыве в СССР по программе «Программа К» радиосвязь и радарные установки были также блокированы на расстоянии до 1000 км. Установлено, что в широком диапазоне частот амплитуда излучения при высотных ядерных взрывах значительно выше, чем при приземных взрывах той же мощности. Регистрация последствий высотного ядерного взрыва возможна на больших (до 10 тысяч км) расстояниях от места взрыва.

С позиций современной классической электродинамики комптоновские модели вызывают серьезные вопросы. Например, почему все комптоновские электроны должны двигаться с релятивистской скоростью синфазно с фронтом гамма-излучения? У комптоновских электронов вектор скорости имеет пространственное распределение, в связи с этим нельзя получить такой короткий фронт нарастания импульса. В электродинамике теоретически нет механизмов получения в данном месте однополярного импульса электрического поля без пространственного разделения зарядов. Но за десятки наносекунд нарастания импульса невозможно получить пространственное разделение зарядов, обеспечивающее эмпирическую напряженность поля. Сама же комптоновская ионизация оставляет всю систему в целом электронейтральной. Кроме этого, ионосфера не имеет резкой границы, поэтому её ионизация рентгеновским излучением будет проходить постепенно по мере продвижения волны излучения, что приведёт к увеличению длительности конечного импульса до нескольких миллисекунд.

Академик Зельдович с учениками тоже пытались решить эту проблему [61], но в литературе нет информации о том, что ими эта проблема была решена. Следовательно, все говорит о том, что в рамках существующей классической электродинамики результаты, полученные при испытаниях по программе «Starfish» и «Программа К», объяснить пока нельзя.

Опасность прогнозов на основе модели комптоновских электронов, исключающей возможность наличия полей импульса в самом космосе, состоит в перспективе новых космических ядерных взрывов. Но если модель неверна, то опора на неё, как в прошлом на предсказания доктора Бете, чревата взрывом, который выведет из строя большое количество спутников. Тогда оправдываться уже будет поздно.

Используем концепцию скалярно-векторного потенциала для искомого объяснения и покажем, что при космическом ядерном взрыве образуются не поля электромагнитного импульса (ЭМИ), а импульсные электрические поля (ИЭП) без магнитного поля. Причем величины полей ИЭП в космосе гораздо больше, чем в атмосфере и на земной поверхности.

В начальный момент термоядерного взрыва температура сгустка плазмы может достигать нескольких сот миллионов градусов. При таких температурах электронный газ уже не является вырожденным и подчиняется распределению Больцмана. Предположим, что температура образовавшегося при взрыве плазменного сгустка в начальный момент составляет $\sim 10^{8} \mathrm{~K}$, а общий вес бомбы и головной части ракеты, выполненных из металла со средней плотностью электронов $\sim 5 \times 10^{22} \mathrm{~cm}^{-3}$, составляет 1000 кг. При 
удельном весе металла $\sim 8$ г/ $\mathrm{cm}^{3}$ общее количество свободных электронов в образовавшейся плазме, в предположении, что все атомы будут однократно ионизированы, составит $\sim 5 \times 10^{27}$. Наиболее вероятную скорость электронов при указанной температуре определим из соотношения:

$$
v=\sqrt{2 k_{B} T / m}
$$

где $k_{b}$ - постоянная Больцмана, а $m$ - масса электрона.

Пользуясь соотношением (16.4) для вычисления приращения скалярновекторного потенциала с учетом лишь членов $\sim v^{2} / c^{2}$, получаем:

$$
\Delta \varphi \cong \operatorname{Nek}_{\bar{b}} T /\left(4 \pi \varepsilon_{0} r m c^{2}\right),
$$

где $e$ - заряд электрона, $r$ - расстояние от центра взрыва до точки наблюдения.

Соответствующая напряженность радиального электрического поля есть

$$
E=N e k_{5} T /\left(4 \pi \varepsilon_{0} r^{2} m c^{2}\right)=\Delta q /\left(4 \pi \varepsilon_{0} r^{2}\right)
$$

где

$$
\Delta q=\mathrm{Nek}_{\bar{b}} T /\left(m c^{2}\right)
$$

есть эквивалентный заряд взрыва (превышение заряда электронного газа по сравнению с его равновесным значением в металле).

Следует отметить, что при разогреве плазмы ионы тоже приобретают дополнительную скорость, однако, поскольку их масса значительно больше, чем масса электронов, приращением их зарядов можно пренебречь.

В соответствии с (21.2) напряженность радиального электрического поля в эпицентре взрыва при заданных выше параметрах составит $\sim 7 \times 10^{5} \mathrm{~B} / \mathrm{M}$. Учитывая экранирующее действие ионосферы, точную начальную температуру плазменного сгустка, массу бомбы и ракетоносителя, в котором ее подрывают, материалы, из которых изготовлены эти элементы, можно получить величины полей, приближающиеся к экспериментальным.

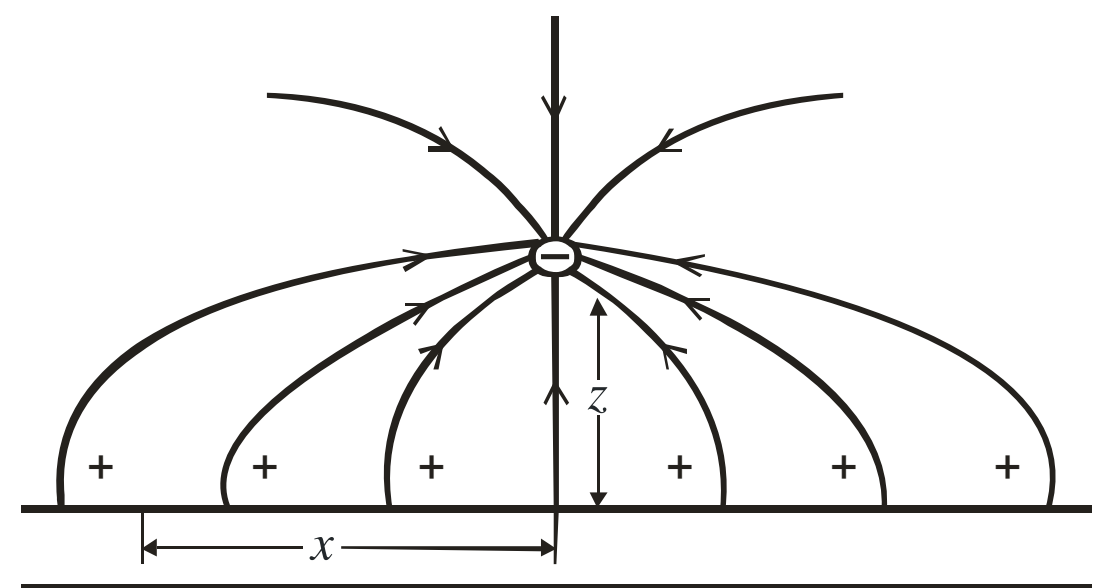

Рис. 39. Отрицательный заряд над безграничной проводящей плоскостью 
Сначала рассмотрим случай отсутствия ионосферы (рис. 39). Для простоты будем считать, что поверхность Земли представляет идеально проводящую безграничную плоскость. Решение задачи распределения полей для заряда, находящегося над такой плоскостью, хорошо известно [4].

Горизонтальная составляющая электрического поля на поверхности такой плоскости равна нулю, а нормальная составляющая равна

$$
E_{\perp}=\frac{1}{2 \pi \varepsilon_{0}} \frac{z q}{\left(z^{2}+x^{2}\right)^{3 / 2}},
$$

где $q$ - величина заряда, $z$ - кратчайшее расстояние от заряда до плоскости, $x$ - расстояние от точки наблюдения до точки пересечения вертикали, опущенной с точки, где находится заряд, на саму плоскость.

Ниже проводящей плоскости электрических полей нет, т.к. заряд индуцирует в ней поверхностную плотность зарядов, полностью компенсирующую горизонтальную и вертикальную составляющие поля заряда в ней. Зависимость плотности поверхностного заряда от величины $x$ имеет вид:

$$
\sigma(x)=\varepsilon_{0} E_{\perp}=\frac{1}{2 \pi} \frac{z q}{\left(z^{2}+x^{2}\right)^{3 / 2}} .
$$

Интеграл $\sigma(x)$ по $x$ есть величина заряда над проводящей плоскостью. Чтобы не пропустить электрические поля заряда $q$, плоскость должна содержать свободные заряды с суммарным зарядом не менее $q$. Рассмотрим с этих позиций экранирующее действие ионосферы (рис. 40). Возникший заряд собирает под собой свободные положительные заряды ионосферы для компенсации создаваемых им в ней полей. Если таких свободных зарядов в ионосфере недостаточно для полной компенсации эквивалентного заряда взрыва, то его поля проникнут сквозь ионосферу. Проникшие поля, в силу экранирующего действия ионосферы, могут быть меньше полей над ней. Всю эту картину можно описать только качественно, потому что точно не известна ни толщина ионосферы, ни степень ее ионизации по высоте.

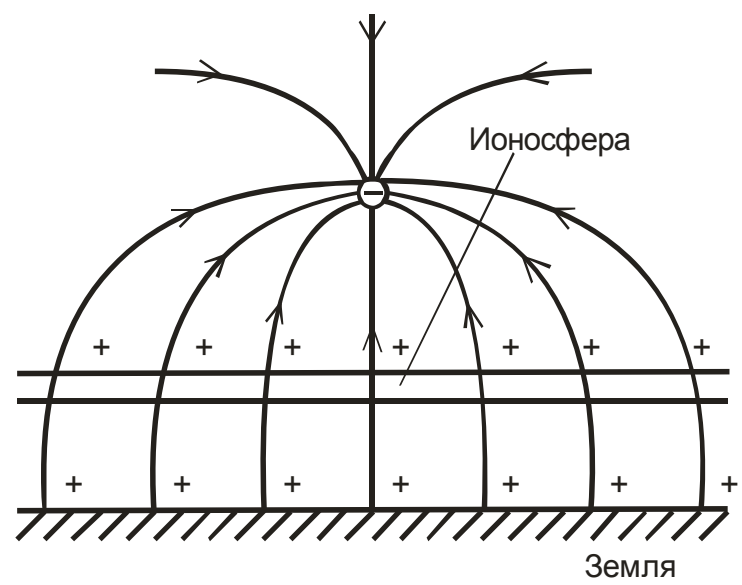

Рис. 40. Отрицательный заряд над поверхностью земли при наличии ионосферы 
Сферичность ионосферы тоже накладывает свои особенности на процесс индукции компенсирующих поверхностных зарядов (рис. 41).

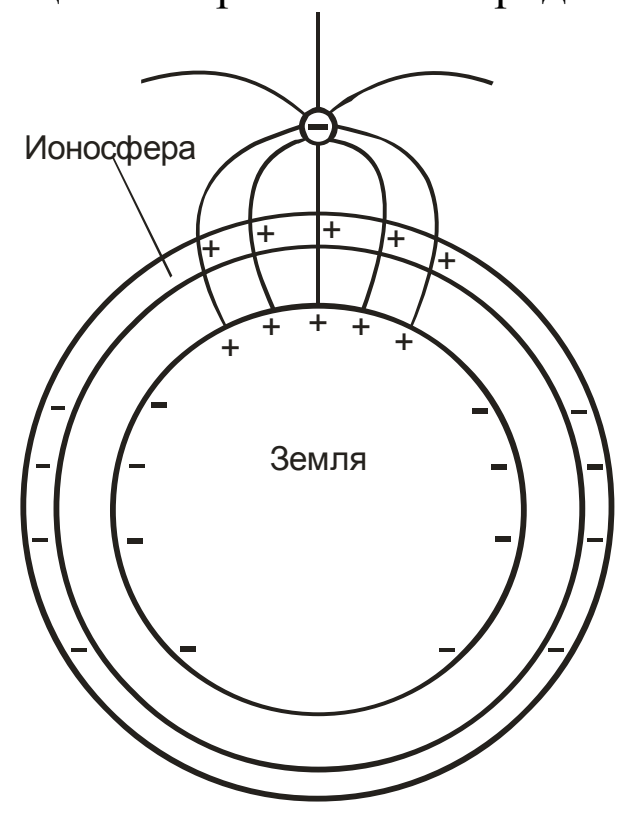

Рис. 41. Отрицательный заряд над поверхностью сферической ионосферы

Стремление возникающего заряда собрать под собой компенсирующие заряды приведет к продольной поляризации значительной части ионосферы. Компенсирующие положительные заряды будут находиться в ионосфере непосредственно в прямой видимости под зарядом, и здесь их будет в избытке, в то время как за пределами прямой видимости в избытке будут отрицательные заряды. И вся система заряд-ионосфера-земля получит дополнительный дипольный момент. Распределение наведенного заряда в ионосфере будет зависеть от высоты, на которой находится заряд, а также от положения Солнца по отношению к заряду, т.к. от его положения зависит степень ионизации ионосферы.

При ядерном взрыве синхронно с электрическими радиальными полями, двигающимися от плазменного сгустка со скоростью света, двигается и фронт рентгеновского излучения. Это излучение также будет ионизировать атмосферу, увеличивая ее проводимость, а это, в свою очередь, увеличит защитные функции атмосферы от проникновения в неё импульсов последующих взрывов, если такие имеются. Кроме того, поскольку отрицательный потенциал плазменного сгустка в начальный момент взрыва очень большой, из сгустка будет временно выброшено какое-то количество электронов, которые также через некоторое время попадут в ионосферу. Частичная нейтрализация электронов, попавших в ионосферу, произойдет, когда положительные ионы плазменного сгустка тоже ее достигнут. Но это касается только ионов, радиальная составляющая скорости которых была направлена в сторону ионосферы. Те же электроны и ионы, радиальная составляющая которых была направлена в сторону от нее, покинут пределы земного тяготения и будут представлять подобие того солнечного ветра, который является следствием испарения солнечной короны или вспышек 
на солнечной поверхности. Сейчас только схематически обрисованы те сложные процессы, которые сопутствуют ядерному взрыву, и предстоит еще большая работа по воссозданию этих процессов для реальных условий. Очевидно, что сделать это можно только численными методами.

Рассмотренная модель говорит о том, что ядерный взрыв приведет не только к возникновению ИЭП в зоне прямой видимости, но и к глобальному возмущению ионосферы. Известно, что взрывы по программе «Starfish» и по программе «Программа К» привели к наличию больших помех радиотехническим и радиолокационным системам. Конечно, электрические поля в космосе, генерируемые таким взрывом, имеют очень большие величины и представляют большую опасность для космических аппаратов.

Теперь вернемся к горизонтальной составляющей электрических полей на земной поверхности, генерируемых при взрыве. Понятно, что эти поля представляют тангенциальную составляющую радиальных полей, идущих от точки взрыва. Именно эти поля и вызывают компенсирующие токи, которые создают компенсирующие поверхностные заряды. Можно вычислить порядок суммарных токов, которые будут иметь радиальную направленность по отношению к эпицентру взрыва. Для этого вычислим суммарный компенсирующий поверхностный заряд на поверхности земли, который должен образоваться при взрыве атомного заряда. Этот заряд равен заряду плазменного сгустка с обратным знаком

$$
q=4 \pi \varepsilon_{0} r^{2} E .
$$

Исходя из реально измеренных вертикальных напряженностей электрических полей в эпицентре взрыва $\left(5.2 \times 10^{4} \mathrm{~B} / \mathrm{M}\right)$, при расстоянии до взрыва 400 км из этой формулы получаем заряд $\sim 10^{6}$ Кл. Значение же по (21.3) составит $\sim 1.2 \times 10^{7}$ Кл. Расхождение, как уже сказано, может быть связано с экранирующим действием ионосферы. Если нарастания импульса электрического поля составляет $\sim 150 \mathrm{Hc}$, то суммарный ток, направленный к эпицентру взрыва, должен составлять $\sim 3 \times 10^{11}$ А. Это значение несколько завышено, потому что компенсирующие заряды притягиваются не к одной точке эпицентра взрыва, а к достаточно обширной области в его окрестности. Но даже если эту величину уменьшить на несколько порядков, то попрежнему величина компенсирующих токов будет очень большой. Теперь понятно, почему на острове Оаху, находящемся на расстоянии 1300 км от места взрыва, сгорели 300 уличных фонарей, а близ Джесказгана в воздушной телефонной линии протяженностью 570 км возникли токи 2.5 кА и сгорели все плавкие предохранители. Даже на силовой кабель протяженностью более 1000 км, соединяющий Алма-Ату и Акмолу, и имеющий бронированный экран из свинца, оплетку из стальной ленты, и находящийся на глубине 0.8 м, возникли такие наводки, что сработали автоматы, отключив электростанцию от кабеля. Конечно, импульс тангенциальных токов, хотя и менее значительный, чем на земной поверхности, будет и в ионосфере, что приведет к ее возмущению в глобальных масштабах.

Опишем формирование ИЭП при взрыве. За время детонации ядерного заряда (несколько наносекунд) образуется плотный сгусток плазмы с тем- 
пературой в несколько десятков и даже сотен миллионов градусов. Этот сгусток генерирует мощное гамма излучение, распространяющееся во все стороны от сгустка со скоростью света. Одновременно генерируется радиальное электрическое поле, тоже распространяющееся в радиальном направлении от сгустка со скоростью света. Радиальные электрические поля ИЭП и гамма-излучение достигают ионосферы одновременно. При дальнейшем движении к поверхности земли, если мощности взрыва для этого достаточно, рентгеновское излучение ионизирует и слои атмосферы, находящиеся ниже ионосферы. Одновременно будет происходить ионизация верхних слоев атмосферы и проникновение в них радиального электрического поля. В ионизированных слоях за счет наличия радиального электрического поля возникнут радиальные токи, которые приведут к расслоению зарядов и к вертикальной поляризации проводящих слоев. Поляризация атмосферы будет иметь место, пока существует радиальное поле и проводимость ионизированного воздуха. Поскольку ионосфера не сможет обеспечить заряд, необходимый для полной компенсации радиального поля плазменного сгустка, эти поля, хотя и в ослабленном виде, будут продолжать распространяться к земной поверхности. Достигнув её, электрические поля создадут мощные радиальные токи. Процесс распространения рентгеновского излучения и радиальных полей через ионосферу приведет к ее дополнительной ионизации и поляризации, а также к появлению импульса тангенциальных токов. Импульс тангенциальных токов в ионосфере будет распространяться на расстояния значительно большие, чем зона видимости взрыва, что приведет к глобальным возмущениям ионосферы.

К моменту прекращения потока жесткого гамма-излучения и ионизации атмосферы часть атмосферы, ионизованной ниже существующей границы ионосферы, перестанет быть проводником, а, следовательно, пространственно-разделенные заряды окажутся запертыми в ней. Запертые электроны по-прежнему будут создавать какую-то статическую разность потенциалов, медленно релаксирующую в меру наличия какой-то остаточной проводимости атмосферы. Следует отметить, что полярность этого поля противоположна полярности первоначального ИЭП, что и наблюдается в действительности. Это значит, что радиальное электрическое поле, наблюдаемое на поверхности земли, сначала будет направлено от земли к месту взрыва, но в какой-то момент времени оно изменит свою полярность.

Теперь понятно, почему после космического ядерного взрыва ещё долго наблюдается остаточное свечение атмосферы под местом взрыва. Это свечение обязано тем электронам, которые на первом этапе развития ИЭП были перемещены из ионосферы в более плотные слои атмосферы, а затем, после прекращения ионизируещего действия гамма излучения, остались запертыми в мало проводящей атмосфере, продолжая её ионизировать.

Таким образом, возникновение ИЭП при ядерном взрыве относится к самому взрыву, а не вторично, и может быть объяснено в рамках концепции скалярно-векторного потенциала. Изучая топологию ИЭП на земной поверхности, можно судить также о последующих процессах поляризации и деполяризации ионосферы, атмосферы и земной поверхности. При взрыве 
в атмосфере сам процесс образования ИЭП и его развитие связаны с наличием атмосферы, а также наличием проводимости у земной поверхности, и это будет также накладывать свои особенности на формирование полей ИЭП. При взрывах в непосредственной близости от земной поверхности эквивалентный заряд облака взрыва будет видеть своё зеркальное отражение под поверхностью земли, образуя электрический диполь. По этой причине область распространения полей ИЭП будет сильно уменьшена, поскольку поля диполя убывают по кубическому закону.

Следует сделать одно замечание по поводу самого термина электромагнитный импульс (ЭМИ), используемого в литературных источниках. Из этого названия следует исключить слово магнитный, т.к. данный процесс представляет распространение только радиальных электрических полей, и в этом случае магнитные поля отсутствуют. Другое дело, что электрические поля могут наводить в окружающих проводящих средах токи, и эти токи будут генерировать магнитные поля, но это уже вторичное явление.

Пока, однако, не рассмотрен существенный вопрос баланса энергий при взрыве. Если считать, что одна тонна тротила эквивалентна $4.6 \times 10^{9}$ Дж, то при взрыве бомбы с тротиловым эквивалентом 1,4 Мт выделяется 6.44 $\times 10^{15}$ Дж. Если время детонации $150 \mathrm{Hc}$, то мощность взрыва составляет $\sim 4 \times 10^{22}$ Вт. Для примера скажем, что мощность излучения Солнца $\sim 3.9 \times 10^{26}$ Вт. Рассмотрим вопрос, куда и каким образом за столь короткое время может быть израсходована энергия, выделенная при таком взрыве.

Согласно уравнению Стефана-Больцмана мощность, излучаемая нагретой поверхностью, пропорциональна четвертой степени ее температуры:

$$
P=\sigma s T^{4}
$$

где $\sigma=5.67 \cdot 10^{-8} \mathrm{Bm} /\left(\mathrm{M}^{2} K^{4}\right)$ - постоянная Стефана- Больцмана, а $s$ - площадь излучающей поверхности.

Если взять начальную температуру плазменного сгустка $\sim 10^{8} \mathrm{~K}$, то при начальном его диаметре 1 м (при этом площадь поверхности сгустка составляет $\sim 3 \mathrm{~m}^{2}$ ) вся энергия взрыва будет излучена за время $\sim 0.4$ нс. Если же взять начальную температуру $\sim 10^{7}$, то это время будет составлять уже 400 нс. Таким образом, следует полагать, что начальная температура сгустка находится где-то между указанными значениями. Длина волны излучения максимального количества энергии определяется законом Вина

$$
\lambda_{\text {макс }}=\frac{0,28975}{T} \frac{\mathrm{cM}}{K} .
$$

Если подставить сюда значение температуры $5 \times 10^{7}$ К, то получим длину волны порядка $6 \AA$, что соответствует жесткому рентгеновскому излучению. По мере охлаждения сгустка его температура начнет падать и $\lambda_{\text {макс }}$ начнет сдвигаться в видимую часть спектра.

Но рассмотренный механизм потерь не является единственным. Поскольку с температурой сгустка однозначно связаны и его электрические поля, то сразу после детонации они будут максимальны, а затем с падением температуры сгустка начнут уменьшаться пропорционально температу- 
ре. Однако энергия, необходимая для их создания, будет падать не так быстро, как энергия необходимая для создания рентгеновского излучения.

Ещё один немаловажный вопрос - сколько электронов покинет плазменный сгусток. Рассмотрим условие электронейтральности плазмы. При превращении металла в плазму меняется не только агрегатное состояние вещества, но и статистика описания электронного газа со статистики ФермиДирака на статистику Больцмана. Когда электронный газ находился в твердом проводнике, то в состоянии электронейтральности на каждый ион приходилось по одному свободному электрону. Определим с точки зрения концепции скалярно-векторного потенциала, какое соотношение должно соблюдаться между электронами и ионами в плазме, чтобы она тоже оставалась электронейтральной. В твердом веществе плотность электронов и ионов одинакова и абсолютные величины их зарядов равны $e N_{e}=e N_{n p}$.

После превращения вещества в плазму общий эквивалентный заряд электронов увеличился на величину (21.3), а у ионов почти не изменился. Теперь уже для соблюдения электронейтральности должно соблюдаться:

$$
N_{e(n \pi)}\left(1+k_{\bar{L}} T /\left(m_{e} c^{2}\right)\right)=N_{n p}
$$

где $N_{e(n л)}$ - равновесное количество электронов в плазме.

Разница между первоначальным и равновесным количеством составляет

$$
\Delta N=N_{n p}\left(1-\left(1+k_{b} T /\left(m_{e} c^{2}\right)\right)^{-1}\right)
$$

Например, при температуре $\sim 10^{8}$ величина в скобках составит примерно 0.13 , т.е. для сохранения электронейтральности плазмы $13 \%$ от общего первоначального количества электронов должны ее покинуть. Назовем это эффектом временно лишних электронов (пока плазма горячая). Теперь проясняются причины появления на поверхности Солнца мощных магнитных полей, особенно при пятнах. Эти поля индуцируются токами, перетекающими между областями плазмы с различной температурой.

Мы рассмотрели поведение статического заряда над проводящей плоскостью. Но в действительности заряд не статический, а живущий всего несколько сот наносекунд. Если в начале координат расположен заряд $Q(t)$, зависящий от времени, то электрические поля, создаваемые им в окружающем пространстве, могут быть найдены из соотношения:

$$
\varphi(r, t)=Q(t)(t-r / c) /(4 \pi \varepsilon r)
$$

которому соответствуют запаздывающие продольное электрические поля:

$$
E(r, t)=Q(t)(t-r / c) /\left(4 \pi \varepsilon r^{2}\right) .
$$

В соответствии с (21.6), (21.7) короткоживущий заряд порождает столь же кратковременный импульс продольных электрических полей, которые распространяются со скоростью света и образуют сферический слой, толщина которого равна времени существования заряда, умноженному на скорость света. Если для нашего случая принять, что время жизни заряда 
составляет полуширину импульса ИЭП (где-то около 100 нс), то толщина этого слоя составит около 30 м. Сферический слой, достигнув сначала ионосферы, а затем земли, наведет там такие же радиальные токи, как если бы статический заряд появился и, просуществовав 100 нс, исчез.

По топологии импульса ИЭП можно судить о температуре плазмы и процессах в ней, причём важен не вид энергии разогрева, а лишь количество свободных электронов, т.е. степень ионизации, зависящая от конечной температуры. Для термоядерного синтеза считается перспективным быстрый разогрев образца мощным лазерным импульсом до высокотемпературной плазмы подобно ядерному взрыву. Образец нужно окружить двумя сферическими проводящими экранами, подключив между ними высокоскоростной осциллограф с высоким входным сопротивлением. Внешний экран следует заземлить. В момент разогрева плазмы возникнет ИЭП. Разность потенциалов между экранами возникнет гораздо раньше, чем материальные частицы плазмы достигнут стенок первого экрана. Изучая топологию записанного импульса, можно судить о временных энергетических процессах разогрева плазмы. Рассчитаем ожидаемую разность потенциалов между экранами в зависимости от температуры и количества свободных носителей зарядов в плазме из (21.5) и (21.7) при $k_{b} T \square m c^{2}$ :

$$
U=N e k_{b} T\left(r_{1}^{-2}-r_{2}^{-2}\right) /\left(4 \pi \varepsilon_{0} m c^{2}\right),
$$

где $r_{1}$ и $r_{2}$ - радиусы наружного и внутреннего экранов соответственно, а $N$ - количество свободных электронов в разогретой плазме.

Факт наличия лишних электронов следует учитывать и при осуществлении управляемого термоядерного синтеза, поскольку это явление должно влиять и на устойчивость плазмы при её разогреве.

\section{§ 22. Теоретические основы гиперконтинуальной физики и гиперконтинуальной электродинамики}

По закону Кулона, модуль $F$ силы $\mathbf{F}$ взаимодействия двух неподвижных (закрепленных) точечных зарядов $q_{0}, q$ в вакууме, направленной вдоль соединяющей их прямой, пропорционален их абсолютным величинам $\left|q_{0}\right|$, $|q|$ и обратно пропорционален квадрату расстояния $r$ между ними:

$$
F=\left|q_{0} q\right| /\left(4 \pi \varepsilon_{0} r^{2}\right)=4 \pi \varepsilon_{0} r^{2} E_{0} E,
$$

где $E_{0}, E$ - модули напряженности электрического поля заряда $q_{0}$ в точке нахождения заряда $q$ и напряженности электрического поля заряда $q$ в точке нахождения заряда $q_{0}$ соответственно.

Если убрать закрепление зарядов $q_{0}, q$, то они придут в движение с соответствующими начальными ускорениями $\mathbf{a}_{0}=\mathbf{F} / m_{0}, \mathbf{a}=\mathbf{F} / m$, направленными вдоль прямой, их соединяющей. Модули этих ускорений равны

$$
a_{0}=F / m_{0}=4 \pi \varepsilon_{0} r^{2} E_{0} E / m_{0} ; a=F / m=4 \pi \varepsilon_{0} r^{2} E_{0} E / m
$$


где $m_{0}, m$ - массы зарядов $q_{0}$ и $q$.

Если перейти в ИСО, движущуюся перпендикулярно прямой, соединяющей заряды, со скоростью $\mathbf{v}$ с модулем $v$, то в этой ИСО поперечные составляющие напряженности увеличатся, в силу чего модули напряженности электрического поля заряда $q_{0}$ в точке нахождения заряда $q$ и напряженности электрического поля заряда $q$ в точке нахождения заряда $q_{0}$ будут в этой ИСО равны уже не $E_{0}, E$, а соответственно

$$
E_{\perp 0}=E_{0} \operatorname{ch}(v / c) ; E_{\perp}=E \operatorname{ch}(v / c) .
$$

В результате, модуль силы взаимодействия зарядов в данной ИСО также возрастет и будет равен уже не $F$, а величине

$$
F_{\perp}=4 \pi \varepsilon_{0} r^{2} E_{\perp 0} E_{\perp}=F \operatorname{ch}^{2}(v / c) .
$$

Так как, согласно принципу относительности, начальные ускорения зарядов при переходе в другую ИСО не меняются, имеем:

$$
a_{0}=F / m_{0}=F_{\perp} /\left(m_{0} \operatorname{ch}^{2}(v / c)\right) ; a=F / m=F_{\perp} /\left(m \operatorname{ch}^{2}(v / c)\right) .
$$

Величины в знаменателях

$$
m_{\perp 0}=m_{0} \operatorname{ch}^{2}(v / c), m_{\perp}=m \operatorname{ch}^{2}(v / c)
$$

назовем поперечными массами зарядов $q_{0}, q$, а обычные массы $m_{0}, m$ можно еще иначе назвать их продольными массами.

Таким образом, в отличие от независящей от скорости продольной массы, поперечная зависит от скорости. Аналогичная ситуация имеет место и в отношении напряженности электрического поля заряда, а именно, в отличие от независящей от скорости продольной составляющей напряженности, поперечная зависит от скорости. При этом выполнено равенство

$$
m_{\perp} / m=\left(E_{\perp} / E\right)^{2}=\operatorname{ch}^{2}(v / c) .
$$

В состоянии продольного покоя $(v=0)$ поперечная масса равна продольной, что позволяет интерпретировать продольную массу, как поперечную массу продольного покоя или просто массу покоя.

Зависимость поперечной массы от скорости естественно ассоциировать с аналогичной зависимостью от скорости нового вида энергии покоя, которую назовем поперечной энергией поперечного покоя:

$$
W_{\perp}=m_{\perp} c^{2} .
$$

В отличие от независящей от скорости энергии покоя

$$
W=m c^{2},
$$

которую можно еще иначе назвать продольной энергией покоя, поперечная энергия поперечного покоя от скорости зависит, причем зависимостью того же вида, что и поперечная масса:

$$
W_{\perp}=W \operatorname{ch}^{2}(v / c)
$$

Подобно ситуации с продольной и поперечной массами, в состоянии продольного покоя $(v=0)$ поперечная энергия поперечного покоя равна продольной энергии покоя, что позволяет интерпретировать продольную 
энергию покоя, как поперечную энергию поперечного и продольного покоя.

Наличие массы $m$ и скорости $\mathbf{v}$ обусловливает существование двух видов энергии: кинетической энергии

$$
T=m v^{2} / 2
$$

(иначе ее можно назвать продольной кинетической энергией) и энергии

$$
T_{\perp}=W_{\perp}-W=\left(m_{\perp}-m\right) c^{2},
$$

которую можно еще иначе назвать поперечной кинетической энергией. Последняя, в свою очередь, обусловливает существование дополнительной по отношению к продольной массе $m$ составляющей поперечной массы $m_{\perp}$, которую назовем кинетической массой:

$$
\tilde{m}=m_{\perp}-m .
$$

Поперечная кинетическая энергия выражается через кинетическую массу:

$$
T_{\perp}=\tilde{m} c^{2} .
$$

Используя зависимость поперечной энергии поперечного покоя от скорости, запишем равенство для поперечной кинетической энергии в виде:

$$
T_{\perp}=W_{\perp}-W=W\left(\operatorname{ch}^{2}(v / c)-1\right) .
$$

Хорошо известно, что для ускорения материальных тел нужно затратить энергию, для чего к ним нужно приложить силу. Выполненная работа переходит в кинетическую энергию движения. При торможении тело отдаёт эту энергию окружающим телам, для чего требуются силы, обратные тем, которые тело ускоряли. Это и есть феномен инерции.

Ясно, что процесс ускорения накапливает в самом теле какой-то вид энергии, который и возвращается потом во внешнюю среду при его торможении. Но ни одна из существующих в настоящее время теорий не даёт ответ на вопрос, что это за энергия, и каким образом она накапливается и

отдаётся. У заряженных тел и у самих зарядов имеются электрические поля, обладающие энергией. Можно было ожидать, что зависимость этих полей от скорости могла бы пролить свет на этот вопрос. В специальной теории относительности (СТО) электрические поля зарядов зависят от скорости, и, казалось бы, эта теория должна была дать ответ на этот интересный вопрос. Но в СТО заряд является инвариантом скорости. Хотя его поле изменяется в процессе ускорения, но это изменение происходит таким образом, что увеличение составляющей поля, нормальной к направлению движения, компенсируется уменьшением продольной составляющей. В результате, поток электрического поля через поверхность, окружающую заряд, не зависит от движения заряда и остаётся постоянным.

Однако в рамках концепции зависимости скалярного потенциала заряда от скорости нормальная составляющая электрического поля увеличивается, в то время как продольная остается неизменной. Такой подход даёт возможность объяснить феномены инерции и кинетической энергии. Все материальные тела состоят из заряженных элементарных частиц, поэтому 
феномены инерции и кинетической энергии тел сводятся к тем же феноменам для частиц. Ввиду идентичности рассмотрения для любых заряженных частиц, без ограничения общности будем частицей считать электрон.

Удельная энергия электрического поля электрона $w=\varepsilon E^{2} / 2$ выражается через напряженность $E=e /\left(4 \pi \varepsilon_{0} r^{2}\right)$. Используя элемент объёма $4 \pi r^{2} d r$, имеем энергию поля покоящегося электрона с зарядом $e$ и радиусом $a$ :

$$
W=\int_{a}^{\infty}\left(e^{2} / 8 \pi \varepsilon_{0} r^{2}\right) d r=e^{2} /\left(8 \pi \varepsilon_{0} a\right) .
$$

При скорости $\mathbf{v}$ нормальная составляющая поля увеличивается:

$$
E_{\perp}=\operatorname{Ech}(v / c) \approx E\left(1+v^{2} /\left(2 c^{2}\right)\right) .
$$

Запишем электрические поля, нормальные к направлению движения в системе координат, представленной на рис. 42 :

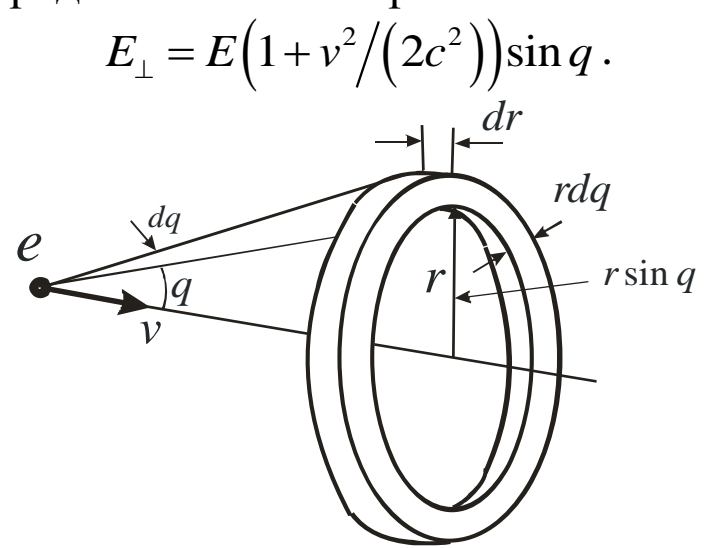

Рис. 42. Элемент объёма $2 \pi r^{2} \sin q d q d r$ для вычисления энергии полей

Тогда энергия полей движущегося электрона запишется в виде:

$$
W_{v}=\left(1+v^{2} /\left(2 c^{2}\right)\right)^{2} \int e^{2} \sin ^{3} q /\left(8 \pi \varepsilon_{0} r^{2}\right) d q d r .
$$

Интегрирование по углу даёт

$$
\int_{0}^{\pi} \sin ^{3} q d q=-\int_{0}^{\pi}\left(1-\cos ^{2} q\right) d(\cos q)=-\cos q+\left(\cos ^{3} q\right) / 3=4 / 3
$$

поэтому имеем:

$$
W_{v}=\frac{4}{3}\left(1+\frac{1}{2} \frac{v^{2}}{c^{2}}\right)^{2} \int_{a}^{\infty} \frac{e^{2} d r}{8 \pi \varepsilon_{0} r^{2}}=\frac{4}{3}\left(1+\frac{v^{2}}{c^{2}}+\frac{1}{4} \frac{v^{4}}{c^{4}}\right) \frac{e^{2}}{8 \pi \varepsilon_{0} a} .
$$

Пренебрегая членом $v^{4} /\left(4 c^{4}\right)$ для нерелятивистских скоростей, имеем:

$$
W_{v}=\left(1+v^{2} / c^{2}\right) e^{2} /\left(6 \pi \varepsilon_{0} a\right) \text {. }
$$

Энергия полей и масса покоя электрона связаны равенством [4]

$$
W=e^{2} /\left(6 \pi \varepsilon_{0} a\right)=m c^{2},
$$

следовательно, дополнительная энергия электрона, связанная с тем, что его поля зависят от скорости, определится соотношением

$$
W_{v}-W=W\left(\operatorname{ch}^{2}(v / c)-1\right) \approx m v^{2} .
$$


Такое же соотношение выполнено для поперечной кинетической энергии:

$$
T_{\perp}=W_{v}-W \approx m v^{2} .
$$

Таким образом, вся дополнительная энергия электрического поля идёт на формирование поперечной кинетической энергии, поэтому их можно отождествлять, считая разными проявлениями одной и той же энергии.

Получился чрезвычайно интересный результат - поперечная кинетическая энергия примерно равна удвоенной продольной кинетической:

$$
T_{\perp} \approx 2 T .
$$

Но не должно ли данное равенство быть точным? Если это на самом деле так, то можно получить более простое равенство, относя продольную и поперечную кинетические энергии к отдельным координатным осям.

Введем индексы $\alpha=\overline{1,3}, \beta=\overline{1,3}$, символ Кронекера $\delta_{\alpha \beta}$. Пусть радиусвектор электрона в относящейся к данной инерциальной системе отсчета системе прямоугольных декартовых пространственных координат с ортами $\mathbf{e}_{\alpha}=\left(\delta_{\alpha \beta}\right)$ имеет вид $\mathbf{x}=\left(x_{\alpha}\right)$. Выберем ориентацию системы пространственных координат так, чтобы орт $\mathbf{e}_{3}$ был коллинеарен вектору скорости $\mathbf{v}=\left(v_{\alpha}\right)$, т.е. $v_{\alpha}=\delta_{\alpha 3} v$. Введем в рассмотрение отнесенные к конкретной оси $\alpha=\overline{1,2}$ пространственных координат (покоординатные) кинетическую массу $\tilde{m}_{1}=\tilde{m}_{2}=\tilde{m}_{\uparrow}$ и поперечную кинетическую энергию $T_{\perp 1}=T_{\perp 2}=T_{\uparrow}$ :

$$
T_{\uparrow}=\tilde{m}_{\uparrow} c^{2}=T_{\perp} / 2 ; \tilde{m}_{\uparrow}=m_{\perp} / 2 .
$$

Таким образом, имеет место простое равенство:

$$
T_{\uparrow}=T .
$$

Если это равенство действительно точное, то тем самым получен новый фундаментальный физический закон, который можно сформулировать следующим образом: покоординатные кинетические энергии для разных пространственных координат равны между собой. Такую ситуацию можно называть кинетическим балансом, а сам закон - законом сохранения кинетического баланса.

Новый закон (если он справедлив) является одним из динамических законов сохранения, наряду с известными еще в классической, нерелятивистской физике законами сохранения энергии, импульса и момента импульса. Эти три известных закона выводятся из требования инвариантности лагранжиана относительно трех групп преобразований координат: закон сохранения энергии выводится из инвариантности лагранжиана относительно группы временных сдвигов (свойство однородности времени), сохранения импульса - пространственных трансляций (свойство однородности пространства), момента импульса - пространственных вращений (свойство изотропии пространства). При этом в классической нерелятивистской физике при рассмотрении пространства и времени преобразования Галилея всегда стояли особняком, о чем упомянул еще Минковский в своем знаменитом докладе «Пространство и время». В силу этого, из требования инвариантности лагранжиана относительно группы преобразова- 
ний Галилея или какой-то подгруппы этой группы никакой динамический закон сохранения не выводится. В СТО преобразования Галилея были заменены преобразованиями Лоренца, и уже из требования инвариантности лагранжиана относительно преобразований Лоренца получается свой динамический закон сохранения, связанный с соответствующими компонентами тензора энергии-импульса. Теперь же пришло время восполнить этот недостающий пробел, введя закон сохранения кинетического баланса.

В лагранжевом формализме классической нерелятивистской физики преобразования Галилея учитываются весьма своеобразно по сравнению с другими группами преобразований координат. А именно, можно выделить два аспекта такого учета. Во-первых, считается, что преобразования Галилея являются одним из проявлений изотропии пространства, вследствие чего выдвигается одно из требований к лагранжиану - он должен зависеть только от абсолютной величины скорости, но не от ее направления. Вовторых, считается, что преобразования Галилея отражают физический принцип относительности Галилея, вследствие чего выдвигается другое требование к лагранжиану - он должен приводить к уравнениям движения, инвариантным относительно данных преобразований. Недостатком такого подхода является то, что пространство и время в классической нерелятивистской физике считаются самостоятельными формами существования материи, не связанными в единое пространство-время. В теории относительности пространство и время считаются соединенными друг с другом в единый пространственно-временной континуум с единой метрикой (псевдоевклидовой в СТО и псевдоримановой с определенной кривизной в ОТО), благодаря чему эту метрику можно ввести непосредственно в лагранжиан, обеспечивая его инвариантность относительно соответствующих преобразований координат типа преобразований Лоренца.

В пространственно-временном гиперконтинууме уже нет единой метрики. Он представляет собой иерархически структурированную совокупность бесконечного множества частных пространственно-временных континуумов со своей собственной метрикой и уровневыми преобразованиями Лоренца. Это не позволяет ввести какую-то метрику единого пространствавремени непосредственно в лагранжиан. Тем не менее, в гиперконтинууме есть преобразования координат, играющие в нем фундаментальную роль, преобразования Галилея, так как они трактуются, как уровневые преобразования Лоренца бесконечно высокого уровня. Тем самым, пространство и время не являются самостоятельными формами существования материи, а соединены в единый гиперконтинуум преобразованиями Галилея, которые, по этой причине, должны играть более фундаментальную роль в лагранжевом формализме.

В группе всех возможных преобразований Галилея можно особо выделить подгруппу тех преобразований Галилея, которые оставляют неизменным модуль скорости, меняя лишь ее направление (изовелоцитарные преобразования Галилея). В классической нерелятивистской физике лагранжиан должен быть инвариантен относительно именно таких преобразований Галилея. Однако при характерном для такой физики независимом рас- 
смотрении пространства и времени подгруппа изовелоцитарных преобразований Галилея фактически отождествляется с группой пространственных вращений. Это и является основанием того, что преобразования Галилея считаются одним из проявлений изотропии пространства. Но применительно к гиперконтинууму такое отождествление вряд ли можно считать оправданным, изотропию гиперконтинуума следует отличать от изотропии пространства, а отражающие изотропию гиперконтинуума изовелоцитарные преобразования Галилея - от отражающих изотропию пространства пространственных вращений.

Таким образом, в гиперконтиннуме кроме требований однородности времени, однородности пространства и изотропии пространства на лагранжиан следует накладывать еще и требование изотропии гиперконтинуума. Тем самым, к инвариантности лагранжиана относительно временных сдвигов, пространственных трансляций и пространственных вращений должна добавиться инвариантность относительно изовелоцитарных преобразований Галилея. Как будет показано ниже, именно инвариантность лагранжиана относительно изовелоцитарных преобразований Галилея приводит к закону сохранения кинетического баланса.

В классической нерелятивистской физике лагранжиан частицы в инерциальной системе отсчета имеет вид:

$$
L=T-U,
$$

где $T=m v^{2} / 2-$ кинетическая энергия, $U-$ потенциальная энергия.

Лагранжиан определяется с точностью до полной производной по времени от произвольной функции координат и времени. Добавление такой функции в лагранжиан не влияет на вид уравнений движения, хотя приводит к другому выражению для энергии частицы. В частности, из лагранжиана можно вычесть некоторую константу, имеющую смысл энергии покоя частицы. Если в качестве такой константы взять энергию полей $W=m c^{2}$, то лагранжиан примет вид:

$$
L=T-W-U .
$$

Такому лагранжиану соответствует полная энергия частицы

$$
W_{T}=(\partial L / \partial v) v-L=m v^{2} / 2+m c^{2}+U=T+W+U .
$$

Следует особо подчеркнуть, что при таком рассмотрении и масса, и лагранжиан, и полная энергия, и кинетическая энергия являются скалярами. При рассмотрении подобного лагранжиана в гиперконтинууме нужно такие скалярные величины заменить на соответствующие векторные:

$$
L_{\alpha}=T_{\alpha}-W-U .
$$

Если разные компоненты вектора лагранжиана не равны между собой, то поворот вектора скорости будет приводить к повороту вектора лагранжиана. Поэтому инвариантность вектора лагранжиана относительно изовелоцитарных преобразований Галилея означает равенство между собой всех трех компонент вектора Лагранжиана, а, следовательно, и всех трех компонент вектора кинетической энергии. Прямолинейное движение с постоянным вектором скорости $\mathbf{v}=\left(\delta_{\alpha 3} v\right)$ имеет место только при $U=0$ и 
$T_{3}=T$. В силу внутренней симметрии движения, выражающейся в его инвариантности относительно перестановки первых двух пространственных координат, имеем: $T_{1}=T_{2}=T_{\uparrow}$. Равенство между собой компонент вектора кинетической энергии теперь означает $T_{\uparrow}=T$. Если продольная кинетическая энергия формируется посредством скорости, то поперечная - посредством приращения поперечной массы. В результате, компоненты входящего в вектор лагранжиана вектора кинетической энергии примут вид:

$$
T_{1}=\tilde{m}_{1} c^{2} ; T_{2}=\tilde{m}_{2} c^{2} ; T_{3}=T=m v^{2} / 2 .
$$

Равенство этих величин есть закон сохранения кинетического баланса.

Таким образом, говоря о величине кинетической энергии, можно не уточнять, о какой именно кинетической энергии идет речь: продольной или покоординатной поперечной; все равно они равны между собой. Так как поперечных взаимно ортогональных направлений всего два, то суммарная поперечная кинетическая энергия равна удвоенной покоординатной поперечной кинетической энергии, она же равна удвоенной продольной кинетической энергии, и она же равна дополнительной энергии, связанной с зависимостью поля от скорости:

$$
W_{v}-W \approx 2 T \text {. }
$$

Тем самым, установлена физическая причина наличия у движущихся материальных тел кинетической энергии, а, следовательно, и их инерционных свойств (как продольных, так и поперечных). Эти свойства связаны с зависимостью скалярного потенциала зарядов, из которых состоят все материальные тела, от относительной скорости зарядов. Рост поперечной кинетической энергии обусловлен ростом поперечной составляющей поля. Энергия приращения поперечной составляющей поля за счет ненулевой скорости движения как раз равна поперечной кинетической энергии.

Установление физической причины инерции и кинетической энергии позволяет установить также и физическую причину зависимости скалярного потенциала электрического заряда от скорости. Оказалось, что данная зависимость является прямым следствием нового закона сохранения кинетического баланса, обусловленного инвариантностью лагранжиана относительно изовелоцитарных преобразований Галилея.

Но установление физической причины зависимости скалярного потенциала электрического заряда от скорости позволяет уточнить конкретный вид такой зависимости, а, значит, и соответствующих зависимостей от скорости напряженности электрического поля и поперечной массы:

$$
\varphi_{\perp}=\varphi \sqrt{1+v^{2} / c^{2}} ; E_{\perp}=E \sqrt{1+v^{2} / c^{2}} ; m_{\perp}=m\left(1+v^{2} / c^{2}\right) .
$$

Уточненные зависимости скалярного потенциала и напряженности электрического заряда от скорости не противоречат транскоординатной формулировке электродинамики, так как формулы (17.71) устанавливают вид транскоординатных производных полевых функций только первого порядка, оставляя открытым вопрос о производных более высокого порядка. В данном же случае формулы (17.71) остаются теми же. 
Введение в физику закона сохранения кинетического баланса ведет к радикальной перестройке не только электродинамики, но и вообще физики в целом, а в частности, механики. Так как настоящая монография посвящена электродинамике, вопросы такого глобального переосмысления физических представлений о мире выходят за пределы ее тематики. Тем не менее, привести один показательный пример все же представляется уместным.

В качестве такого примера рассмотрим динамику равномерного вращательного механического движения вокруг своей оси однородного цилиндра радиуса $R$ с модулем угловой скорости $\omega$. Если в состоянии покоя такой цилиндр имеет массу $m$, то в состоянии вращения его масса в направлении оси увеличится до некоторой величины $m_{\perp}$. Это означает, что сила $\mathbf{F}$, действующая в направлении оси вращающегося цилиндра (она может иметь различную физическую природу, включая гравитационную), способна придать ему в этом направлении ускорение $\mathbf{F} / m_{\perp}$, которое будет по модулю меньше в $m_{\perp} / m$ раз того ускорения $\mathbf{F} / m$, которое сообщает та же самая сила цилиндру в отсутствие его вращения.

Такой коэффициент пропорциональности определяется формулой

$$
m_{\perp} / m=\left(2 / R^{2}\right) \int_{0}^{R} r\left(1+\omega^{2} r^{2} / c^{2}\right) d r=1+\omega^{2} R^{2} /\left(2 c^{2}\right) .
$$

Рассмотренный физический эффект обеспечивает увеличение инертности вращающихся тел, однако, до настоящего времени этот физический феномен не имел удовлетворительного теоретического объяснения. Теперь же не только прояснилась физическая природа этого явления, но и получены соответствующие расчетные формулы. Хотя эффект и очень мал, но при соответствующем совершенствовании техники эксперимента он может быть использован для экспериментального подтверждения закона сохранения кинетического баланса. Из этого закона вытекает электродинамика, в основе динамических законов которой лежит зависимость скалярного потенциала электрического заряда от скорости. И из этого же закона вытекает механика больших (релятивистских) скоростей, в основе которой лежит другая (гиперконтинуальная) формулировка второго закона Ньютона.

Пусть сила $\mathbf{F}$ передает материальной точке массой $m$, движущейся со скоростью $\mathbf{v}$, ускорение $\mathbf{a}$. Тогда второй закон Ньютона в классической нерелятивистской физике не содержит скорости и записывается в виде

$$
\mathbf{F} / m=\mathbf{a} \text {. }
$$

Однако в пространственно-временном гиперконтинууме эта формула выполняется лишь приближенно в случае малых (нерелятивистских) скоростей, то есть при $v<<c$, так как в гиперконтинууме различаются продольная масса $m$ и поперечная масса

$$
m_{\perp}=m\left(1+v^{2} / c^{2}\right) .
$$

Продольное и поперечные направления здесь и далее определяются по отношению к направлению вектора скорости $\mathbf{v}$. 
Для удобного представления второго закона Ньютона в пространственно-временном гиперконтинууме целесообразно использовать исчисление кватернионов с набором мнимых единиц $\mathbf{i}, \mathbf{j}, \mathbf{k}$. Положим орты $\mathbf{e}_{1}, \mathbf{e}_{2}, \mathbf{e}_{3}$ системы прямоугольных декартовых пространственных координат данной инерциальной системы отсчета равными:

$$
\mathbf{e}_{1}=\mathbf{i}, \mathbf{e}_{2}=\mathbf{j}, \mathbf{e}_{3}=\mathbf{k} .
$$

При этом радиус-вектор $\mathbf{x}$ оказывается чисто векторным кватернионом, то есть кватернионом с нулевой действительной частью)

$$
\mathbf{x}=x_{1} \mathbf{i}+x_{2} \mathbf{j}+x_{3} \mathbf{k} .
$$

Сила $\mathbf{F}$ и ускорение а раскладываются на соответствующие взаимно ортогональные продольные $\mathbf{F}_{\uparrow}, \mathbf{a}_{\uparrow}$ и поперечные $\mathbf{F}_{\perp}, \mathbf{a}_{\perp}$ составляющие:

$$
\mathbf{F}=\mathbf{F}_{\uparrow}+\mathbf{F}_{\perp} ; \mathbf{a}=\mathbf{a}_{\uparrow}+\mathbf{a}_{\perp},
$$

причем $\mathbf{F}, \mathbf{a}, \mathbf{F}_{\uparrow}, \mathbf{a}_{\uparrow}, \mathbf{F}_{\perp}, \mathbf{a}_{\perp}, \mathbf{v}$ подобно радиусу-вектору $\mathbf{x}$ также являются чисто векторными кватернионами.

Кватернионы продольной и поперечной составляющих ускорения выражаются через кватернионы ускорения и скорости следующим образом:

$$
\mathbf{a}_{\uparrow}=(\operatorname{Re}(\mathbf{a} / \mathbf{v})) \mathbf{v} ; \mathbf{a}_{\perp}=\mathbf{a}-(\operatorname{Re}(\mathbf{a} / \mathbf{v})) \mathbf{v} .
$$

C учетом этих двух кватернионов запишем второй закон Ньютона в пространственно-временном гиперконтинууме на языке кватернионов:

$$
\mathbf{F}=m(\operatorname{Re}(\mathbf{a} / \mathbf{v})) \mathbf{v}+m_{\perp} \mathbf{a}-m_{\perp}(\operatorname{Re}(\mathbf{a} / \mathbf{v})) \mathbf{v} .
$$

Выражая поперечную массу через продольную, окончательно получим:

$$
\mathbf{F} / m=\left(1+v^{2} / c^{2}\right) \mathbf{a}-\left(v^{2} / c^{2}\right)(\operatorname{Re}(\mathbf{a} / \mathbf{v})) \mathbf{v}
$$

При $v<<c$ эта формула переходит в традиционную формулу для данного закона. Однако в общем случае можно констатировать даже несовпадение направлений векторов силы и ускорения.

\section{§ 23. Особенности математического аппарата классической электродинамики}

Основной математический аппарат электродинамики - векторный анализ. Сила, скорость, ускорение, электрическое поле и ток - векторные величины с наглядным физическим смыслом. Приведём несколько примеров векторных величин без него (назовём их векторами-фантомами).

Вращение диска с угловой скоростью $\omega$ изображают вектором, направленным по оси вращения диска и приложенным к его центру. Механический момент тоже направлен по оси вращения, приложен к центру плоскости вращения и равен произведению радиальной скорости на радиус. Магнитный дипольный момент для кругового тока равен произведению тока на площадь обтекаемого им круга. Этот вектор направлен по оси вращения 
круга, приложен к его плоскости. Такие вектора вводятся по договоренности без какого-либо физического смысла, как, например, вектор скорости.

Вектор, представляющий ротор, вводится следующим образом:

$$
\operatorname{rot} \mathbf{a}=\left(\begin{array}{ccc}
\mathbf{i} & \mathbf{j} & \mathbf{k} \\
\partial / \partial x & \partial / \partial y & \partial / \partial z \\
a_{x} & a_{y} & a_{z}
\end{array}\right) .
$$

Чтобы выяснить геометрический смысл ротора, рассмотрим твердое тело, которое вращается с угловой скоростью $\omega$ вокруг оси $z$. Тогда линейная скорость тела $v$ в точке $(x, y, z)$ будет численно равна

$$
v=\omega r=\omega \sqrt{x^{2}+y^{2}} \text {, }
$$

а слагающие её по осям для правовинтовой системы координат равны

$$
v_{x}=-v y / \sqrt{x^{2}+y^{2}}=-\omega y, v_{y}=-v x / \sqrt{x^{2}+y^{2}}=-\omega x, v_{z}=0 .
$$

Слагающие вектора rot $\mathbf{v}$ при этом определятся соотношениями:

$$
\operatorname{rot}_{x} v=\operatorname{rot}_{y} v=0, \operatorname{rot}_{z} v=\partial v_{y} / \partial x-\partial v_{x} / \partial y=2 \omega .
$$

И снова получен вектор, направленный параллельно оси вращения и нормальный к плоскости вращения. Этот вектор тоже вводится по договорённости и какого-либо физического смысла не имеет. Те же рассуждения можно распространить и на векторное произведение.

Итак, при использовании векторного исчисления для описания физических явлений вводятся два типа векторов. Первый - реальные физические вектора, характеризующие саму физическую величину с учётом её величины и направления (например, вектора силы, скорости, ускорения, напряженности электрического поля и тока). Второй - представимые операциями ротора или векторного произведения. Эти вектора не представляют физические величины и вводятся по договорённости, являясь векторамифантомами. Именно к вектору такого типа и относится магнитное поле.

Магнитное поле вводят или при помощи ротора электрического поля

$$
\partial \mathbf{H} / \partial t=-\mu^{-1} \operatorname{rot} \mathbf{E},
$$

или как ротор векторного потенциала

$$
\mathbf{H}=\operatorname{rot} \mathbf{A}_{H} .
$$

Это значит, что магнитное поле не является физическим полем, а есть некий не имеющий физического смысла и вводимый по договорённости векторный символ. При записи уравнений Максвелла ротор от магнитного поля приравнивают полному току

$$
\operatorname{rot} \mathbf{H}=\operatorname{rot} \operatorname{rot} \mathbf{A}_{H}=\mathbf{j}_{\Sigma}
$$

и получается так, что ротор от вектора $\mathbf{H}$, который вводят по договорённости, даёт реальный физический вектор плотности тока. Таким образом, вектор магнитного поля представляет типичный вектор-фантом.

Другой пример - сила Лоренца равна векторному произведению реального вектора скорости движущегося заряда и магнитного поля 


$$
\mathbf{F}=\mu[\mathbf{v} \times \mathbf{H}] .
$$

Опять получается так, что операция векторного произведения, которая сама по себе физического смысла не имеет, с участием реального вектора и вектора фантома даёт реальную физическую силу с учётом её величины и направления. В этом и состоит смысл введения в векторном исчислении таких операций, как ротор и векторное произведение. Итак, аппарат векторного исчисления электродинамики представляет смесь реальных физических векторов и векторов-фантомов, отношения между которыми регулируются при помощи, в том числе, и указанных операций.

Выше была показана возможность построения электродинамики без векторов-фантомов. При этом в её основу должно быть положено такое фундаментальное понятие, как электрическое поле, которое является градиентом скалярного потенциала, а также признание зависимости электрического поля заряда от параметров его движения. По сути дела, это революция в электродинамике. Она лишает магнитное поле прав физического поля и ставит во главу классической электродинамики зависимость электрического поля заряда от нулевой, первой и второй производных по времени радиус-вектора заряда, то есть от его координат, скорости и ускорения.

Понятие магнитного поля как реального вектора существует с тех пор, как учёные заметили организованное поведение железных опилок вблизи магнитов или кольцевых токов. Оно казалось почти очевидным следствием действия какого-то силового вектора, названного магнитным полем. Однако очевидное не всегда реально. Оказывается, что такое поведение железных опилок связано не с магнитным полем, как физическим вектором, а с наличием у микроскопических кольцевых токов в отдельных атомах потенциальной энергии по отношению друг к другу, которая для случая устойчивого равновесия всегда стремится к минимуму.

\section{§ 24. Механическая электризация}

Землетрясения сопровождаются возникновением электрических явлений, которые до настоящего времени не нашли своего объяснения. В 1847 г. перед землетрясением в Синсю (Япония) на фоне тёмного неба возникло вращающееся огненное облако, движущееся в сторону горы Идуна, и исчезло, достигнув её. В 1911 г. накануне землетрясения в Германии в безоблачном небе появился огненный шар, а 26 ноября 1930 г. перед землетрясением на полуострове Идзу (Япония) в небе появились полосы северного сияния. Пережившие ашхабадскую трагедию 1948 г. рассказывают, что накануне землетрясения они увидели летящую по небу дугу из электрических разрядов, затем, сразу же после порыва ветра, раздался первый подземный толчок. Во время ташкентского землетрясения 1966 г. из-под земли вырвался гигантский светящийся факел, стремительно поднялся вверх и растворился в воздухе. А при сверхмощном Тянь-Шанском землетрясении 1976 г. световые вспышки наблюдались за сотни километров от эпицентра. 
Землетрясение начинается с разрыва и перемещения горных пород в очаге (гипоцентре) [62-65] глубиной обычно не больше 100 км, но иногда до 700 км. В одних случаях пласты земли по разным сторонам разлома надвигаются друг на друга. В других - земля по одну сторону разлома опускается, образуя сбросы. Подводные землетрясения вызывают цунами - длинные волны, порождаемые мощным воздействием на всю толщу воды в океане, во время которых происходит резкое смещение (поднятие или опускание) участка морского дна. Цунами образуются при землетрясении любой силы, но большой силы достигают при сильных землетрясениях. Резкое перемещение больших масс земли в очаге землетрясения сопровождается механическим ударом с энергией, многократно превосходящей энергию атомных взрывов [66], а также колоссальными механическими напряжениями и разрывами пластов породы. От очага землетрясения распространяются сейсмические волны, которые также характеризуются периодическим механическим сжатием и растяжением пластов и горных пород. В отдельных случаях землетрясения сопровождаются возникновением молний.

Предположение о том, что электрические явления, сопровождающие землетрясения, могут быть связаны с механическими процессами в пластах породы, подтверждается полученными экспериментальными данными.

Так, возникновение ударных механических нагрузок также должно приводить к возникновению на изолированном металлическом образце импульсного потенциала. Схема установки приведена на рис. 43.

К внешнему экрану при помощи широкой горловины подвешена внутренняя ёмкость. Для устранения гальванического контакта между внешним экраном и внутренней ёмкостью горловина имеет разрез. Разрозненные части горловины соединены изолирующими прокладками, обозначенными на рисунке короткими чёрными отрезками линий. Внутренняя ёмкость изготовлена из алюминия в виде цилиндра, торцевые стенки которого выполнены в виде полусфер.

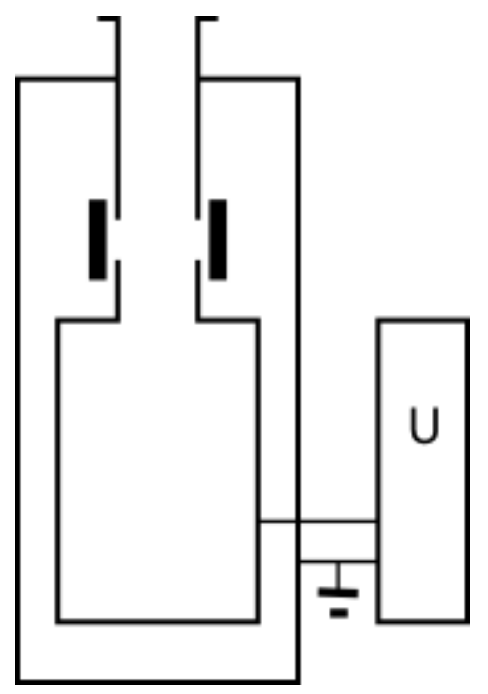

Рис. 43. Схема установки для исследования возникновения импульсов электрического поля при ударных нагрузках 
Такая конструкция торцевых стенок необходима для предотвращения их сильной деформации при взрывах во внутренней ёмкости. Общий вид и составные части установки для исследования динамических нагрузок на алюминиевую колбу показаны на рис. 44, 45. При вбрасывании в горловину стержня весом 200 г с высоты 1 м от дна колбы между внешним экраном и колбой наблюдается импульс напряжения (рис. 46). Для избежания дополнительных импульсов при боковом падении стержня после удара его конца о дно колбы, боковая поверхность стержня обмотана мягкой тканью. Данные этого эксперимента соответствуют полученным с медной колбой, когда её растяжение приводило к отрицательному потенциалу на колбе. При ударе конца стержня о дно колбы также локально деформируется её дно (растяжение в месте удара).

При взрыве внутри алюминиевой колбы небольшого заряда наблюдается импульс напряжения (рис. 47). Разнополярные повторяющиеся импульсы следствие растяжения и сжатия стенок колбы при многократном отражении ударной волны. Если в алюминиевой колбе периодически сжимается и растягивается механически и электрически изолированная от нее пружина, то изменение потенциала на колбе также периодическое. К хлопчатобумажному шнуру, выходящему наружу колбы, прикреплена пружина, на которой подвешен груз (рис. 48). Если к концу шнура приложить периодическую силу на частоте резонанса, можно добиться периодической деформации пружины на этой частоте при почти неизменном положении груза. Если внутри колбы разорвать тонкую медную проволоку, то между колбой и наружным экраном также наблюдается импульс напряжения (рис. 49).

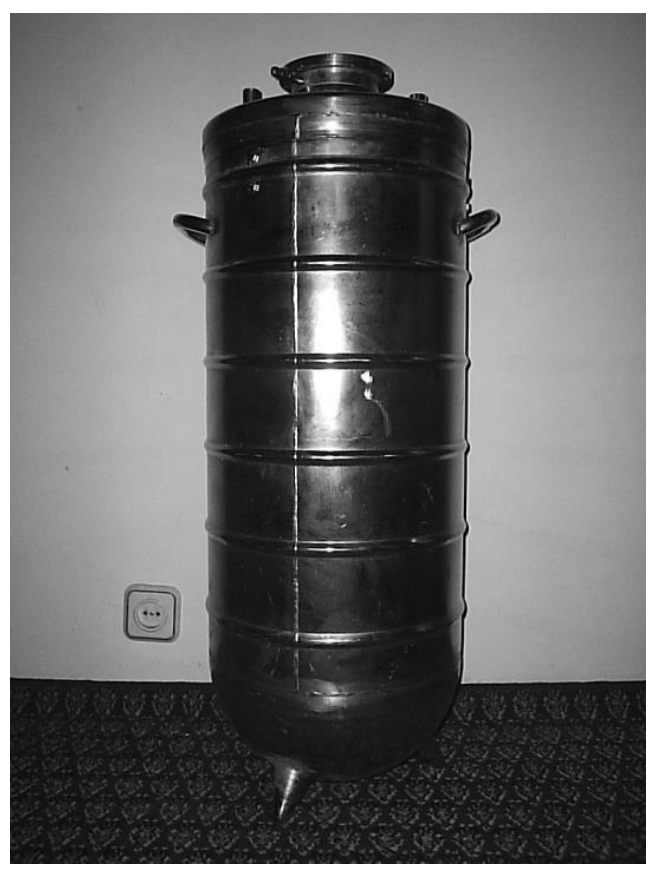

Рис. 44. Общий вид установки для исследования динамических нагрузок

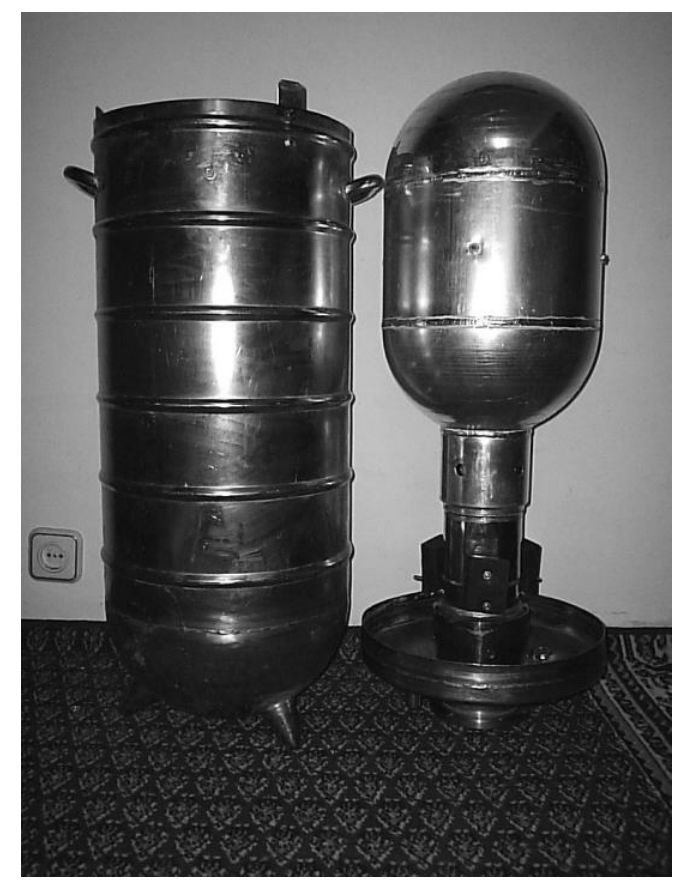

Рис. 45. Вид установки в разобранном виде 


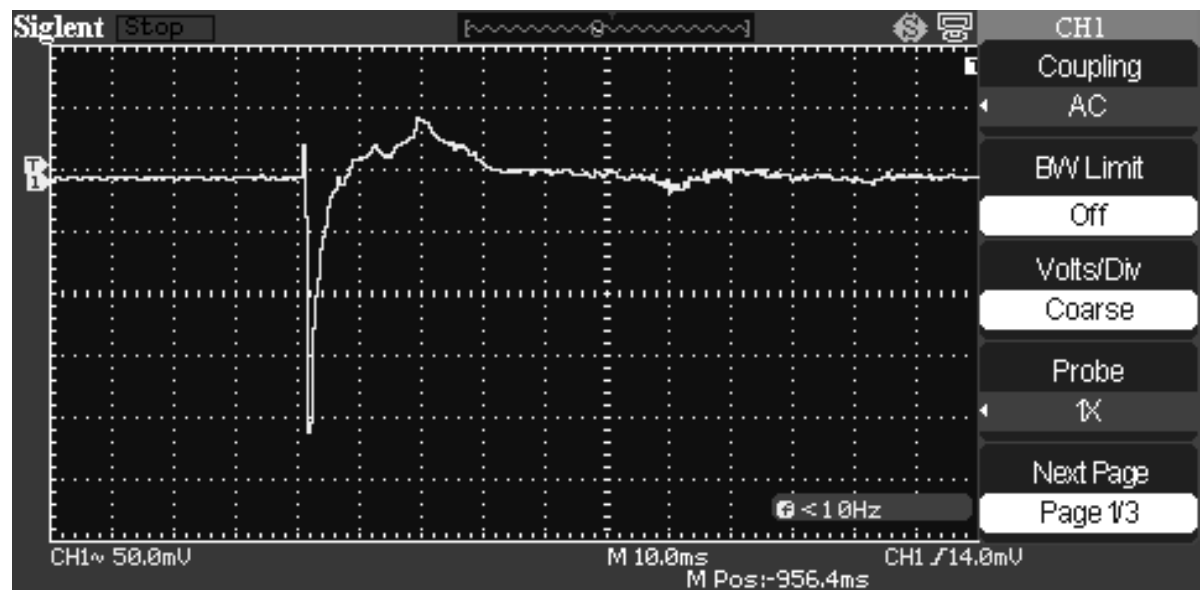

Рис. 46. Импульс после падения стержня на дно внутренней ёмкости

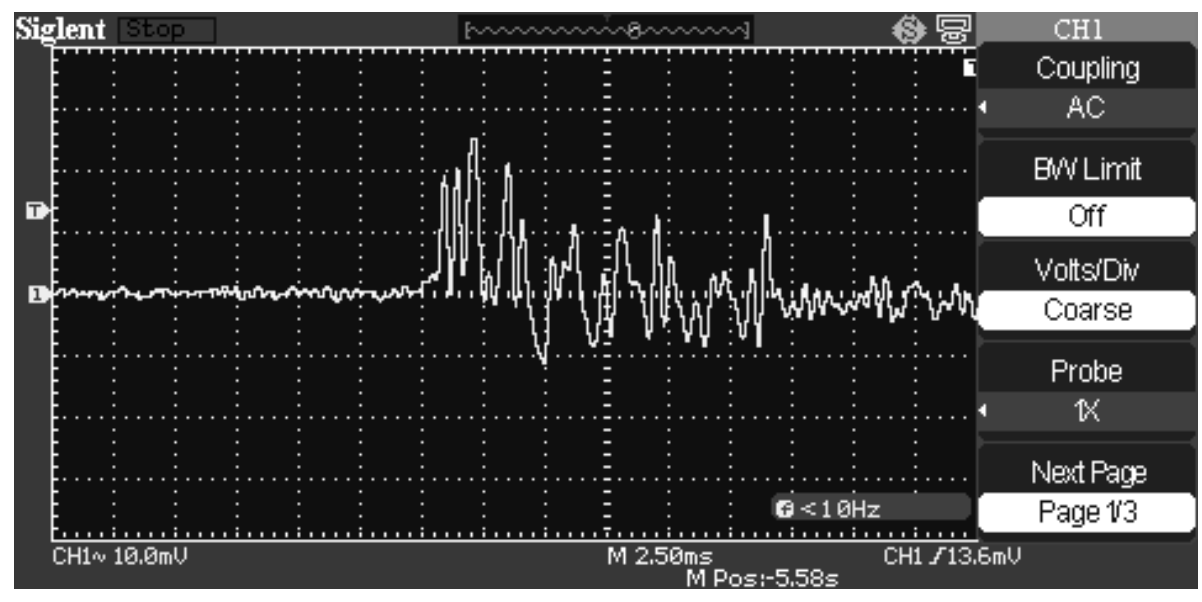

Рис. 47. Импульсы напряжения при взрыве в алюминиевой колбе

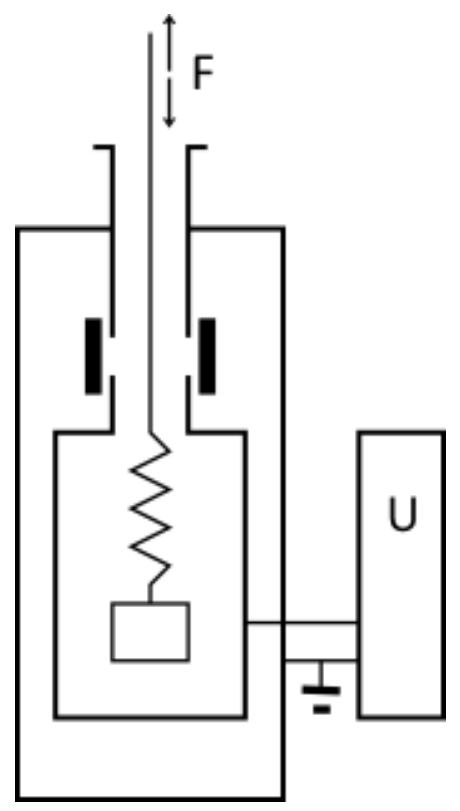

Рис. 48. Схема эксперимента с пружиной внутри колбы

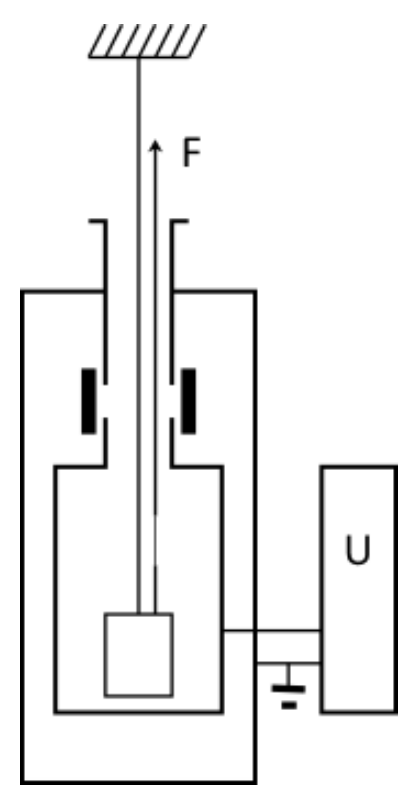

Рис. 49. Эксперимент по разрыву в колбе медной проволоки 
Внутри колбы на хлопчатобумажном шнуре подвешен груз. Параллельно этому шнуру есть другой шнур, в разрыве которого закреплён отрезок медной проволоки диаметром 0.3 мм. Зависимость электрического потенциала на колбе при эксперименте по схеме рис. 48 показана на рис. 50. Импульс при разрыве проволоки между колбой и внешним экраном при эксперименте по схеме рис. 49 показан на рис. 51. Отрицательная часть импульса соответствует растяжению проволоки, предшествующему разрыву. Положительная - релаксации деформационного напряжения в двух частях разорванной проволоки. Таким образом, как механическая деформация проволоки, так и её разрыв сопровождается возникновением унитарного заряда внутри колбы.

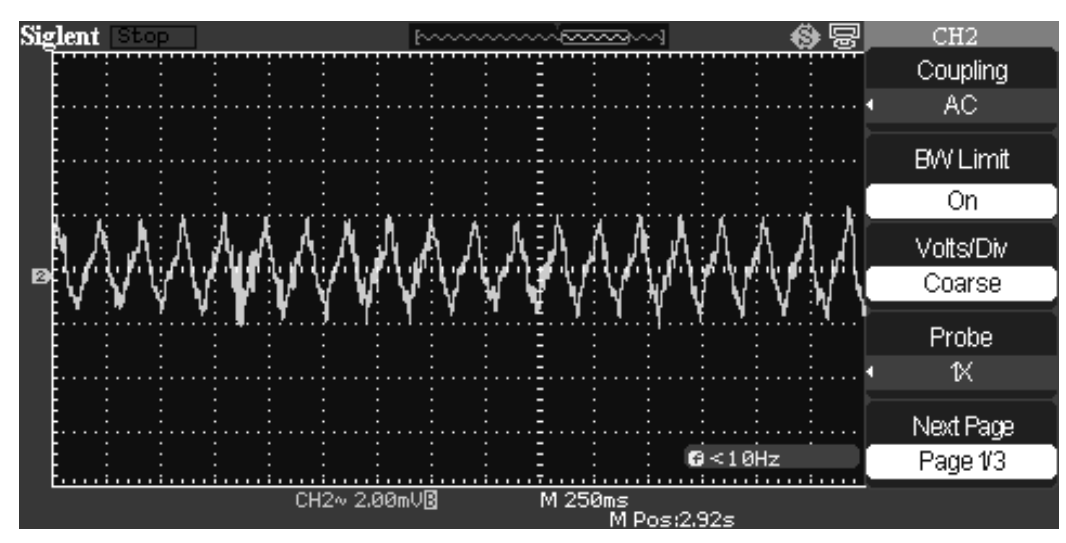

Рис. 50. Периодическое изменение потенциала на колбе при периодическом сжатии и растяжении пружины.

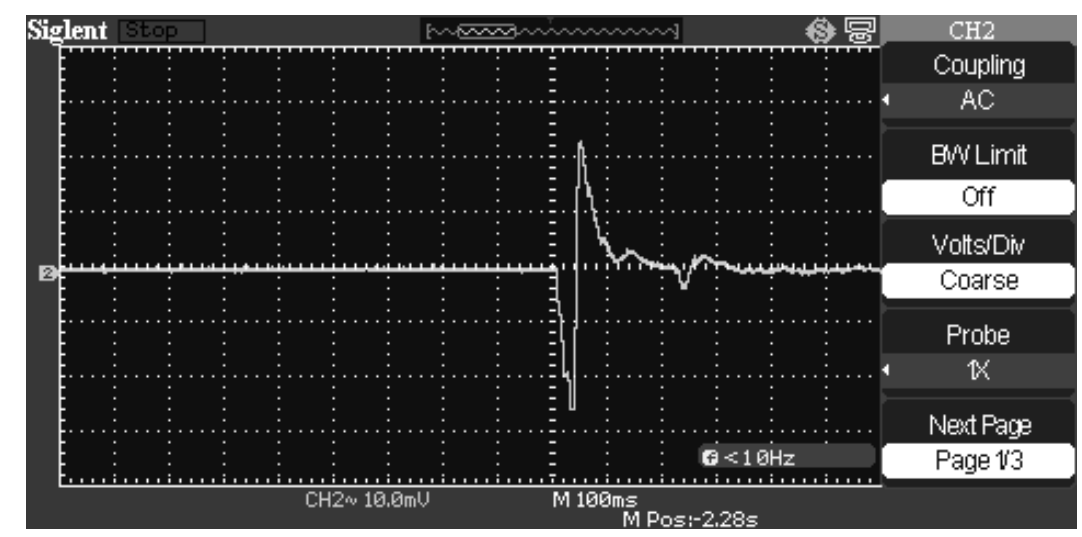

Рис. 51. Импульс, полученный при разрыве медной проволоки

Электризация возникает и при механических деформациях диэлектриков по методике рис. 43. Осциллограммы при разрыве нити из шёлка и из хлорвинила приведены на рис. 52, 53, а при периодических механических нагрузках шнурка, сплетённого из синтетических волокон, - на рис. 54. 


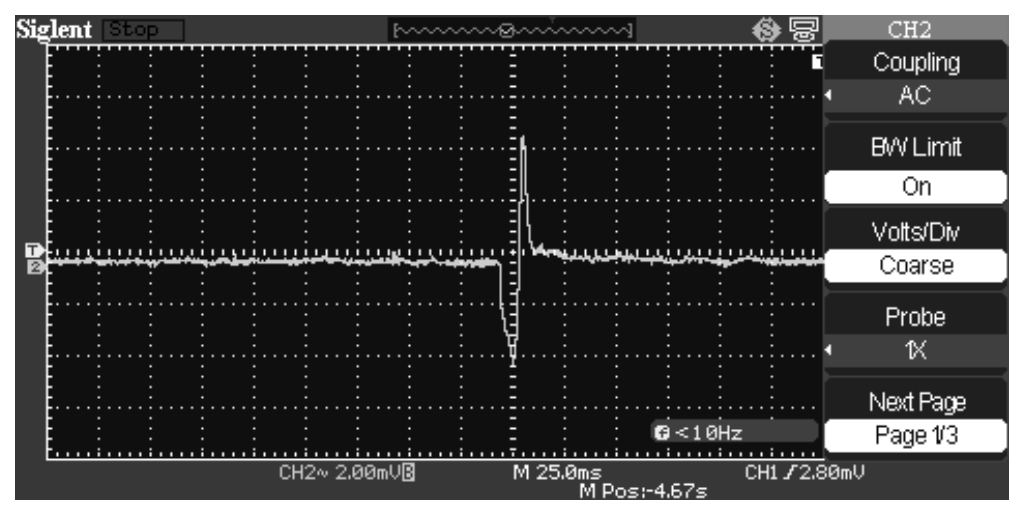

Рис. 52. Осциллограмма, наблюдаемая при разрыве шелковой нити

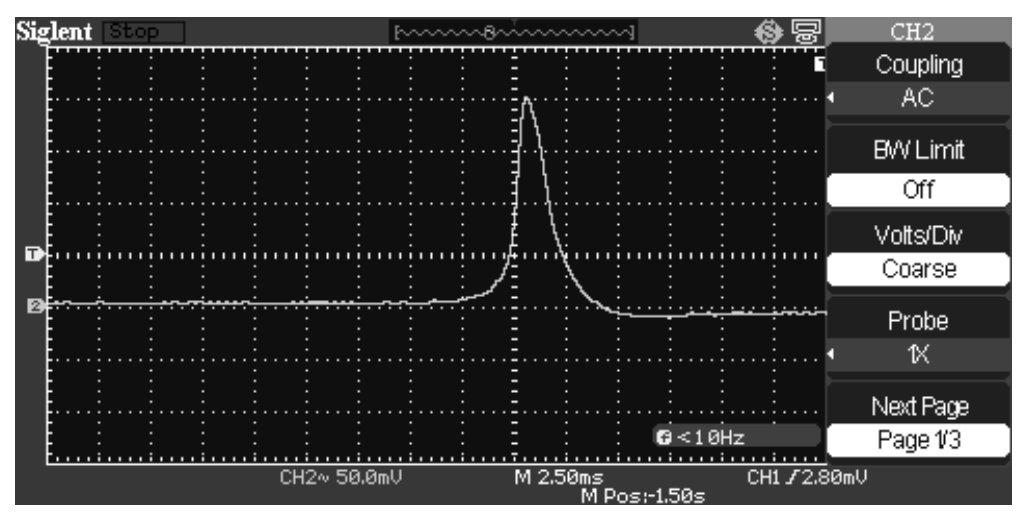

Рис. 53. Осциллограмма, наблюдаемая при разрыве нити из хлорвинила

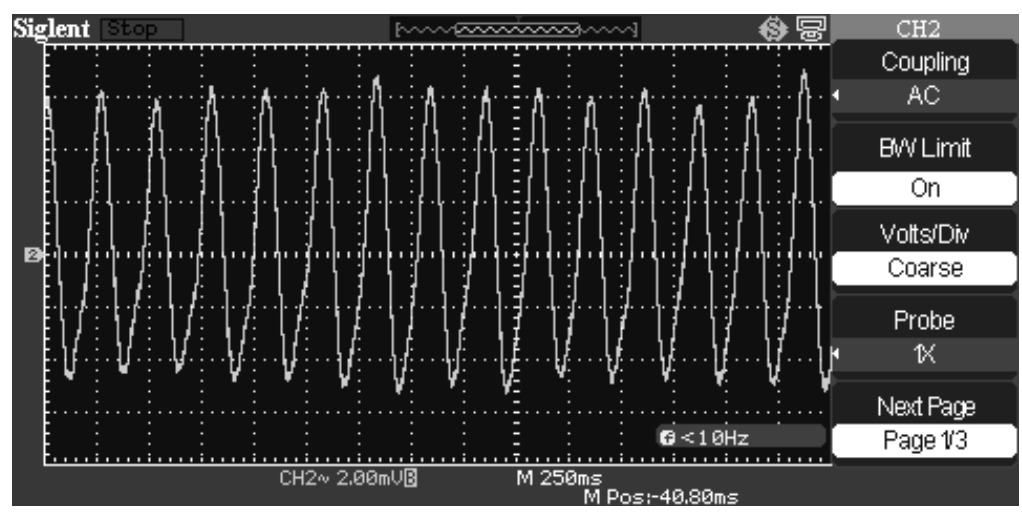

Рис. 54. Осциллограмма, наблюдаемая при приложении к шнурку периодических механических нагрузок

Полученные экспериментальные данные говорят о том, что сжатием, растяжением или разрушением проводников и диэлектриков в клетке Фарадея можно получить в ней унитарный заряд разных знаков, поля которого свободно проникают через ее металлический экран. Трение между отдельными нитями даёт такой же эффект, о чём свидетельствуют эксперименты со шнурком, выполненном из таких нитей. Электризация образцов при их механических деформациях наблюдалась и в работах $[67,68]$. Такие свойства проводников и диэлектриков ранее не были известны. 


\section{ЧАСТЬ II. ДРУГИЕ НОВЫЕ ГИПОТЕЗЫ, ЭКСПЕРИМЕНТАЛЬНЫЕ РЕЗУЛЬТАТЫ И ТЕХНИЧЕСКИЕ РЕШЕНИЯ}

\section{ГЛАВА 5. ДРУГИЕ ГИПОТЕЗЫ, ЭКСПЕРИМЕНТАЛЬНЫЕ РЕЗУЛЬТАТЫ И ТЕХНИЧЕСКИЕ РЕШЕНИЯ}

\section{§ 25. Являются ли лазер и квантовый генератор квантовыми генераторами?}

Лазеры считаются квантовыми генераторами. Известно, что лазерное излучение обладает высокой когерентностью и направленностью. Известный в радиотехнике принцип построения излучающих систем с такими характеристиками - использование большого количества элементарных фазированных излучателей, расположенных в определённом порядке. Называются такие системы фазированными решетками (ФАР). Чем больше элементарных излучателей используется и чем на большем пространстве они расположены, тем большую направленность и мощность можно получить. Для получения высокой направленности линейные размеры системы должны быть значительно больше длины излучаемой волны.

В рабочем веществе лазера тоже всегда содержится громадное количество элементарных излучателей - атомов или молекул рабочего вещества. В твердотельных лазерах, например, рубиновых, излучающие атомы (атомы хрома) тоже расположены в кристалле рабочего вещества в строго определённом порядке. Если такие атомы каким-либо образом синхронно возбудить так, чтобы их колебания были фазированы, как в ФАР, то с радиотехнической точки зрения такая система может давать очень узконаправленное излучение, т.к. количество излучателей очень велико, а длина излучаемой волны гораздо меньше линейных размеров поля излучения. Но как возбудить атомы? Один из таких способов - ударное возбуждение, когда рабочее вещество лазера облучают коротким импульсом от лампы вспышки, как это делается в рубиновом лазере. Но при помощи лампы вспышки нельзя возбудить атомы так, чтобы фаза их колебаний соответствовала условиям ФАР. Для такой селекции служит внешний макроскопический резонатор. Условие указанной селекции - совпадение одной из резонансных мод внешнего резонатора с частотой собственных колебаний атомов хрома. Но этого недостаточно. Решетку ФАР составят только атомы хрома, расположенные в местах, где их фаза колебаний совпадает с фазой колебаний макроскопического резонатора. Но в общем количестве возбуждённых атомов таких не много, всего несколько процентов от общего количества. Поэтому КПД рубинового лазера не высок.

Следовательно, рубиновый лазер работает по всем законам классической электродинамики и радиотехники, и ничего в нём квантового нет, хотя название у него очень красивое - двухуровневый квантовый генератор. 
Многоуровневые квантовые генераторы, излучающие опусканием вниз последовательно через несколько уровней квантов с более высоких уровней, работают по радиотехническим законам нелинейных параметрических систем. Происходит или параметрическое усиление, или параметрическая генерация согласно соотношениям Менли-Роу - энергетическим соотношениям, характеризующим взаимодействие колебаний или волн в нелинейных системах с сосредоточенными или распределёнными параметрами:

$$
\sum_{m=1}^{\infty} \sum_{n=-\infty}^{\infty} \frac{m P_{m n}}{m \omega_{1}+n \omega_{2}}=0 ; \quad \sum_{m=-\infty}^{\infty} \sum_{n=1}^{\infty} \frac{n P_{m n}}{m \omega_{1}+n \omega_{2}}=0,
$$

где $P_{m n}-$ изменение мощности на комбинационной частоте $m \omega_{H}+n \omega_{C} ; \omega_{1}$, $\omega_{2}$ - частоты исходных колебаний (волн). Отношение $\omega_{2} / \omega_{1}$ должно быть иррационально, поскольку в противном случае, возможно выразить все частоты как гармоники одной фундаментальной частоты.

Соотношения Мэнли-Роу справедливы для системы с произвольной реактивной нелинейной связью. В совокупности с законами сохранения энергии и импульса, они определяют характер нелинейного взаимодействия волн (колебаний) и позволяют рассчитать максимальную эффективность преобразователя частоты на реактивной нелинейности.

Пусть в единицу времени появляется или исчезает $A_{m n}$ квантов комбинационной частоты. Тогда мощность на этой частоте равна

$$
P_{m n}=A_{m n} \cdot \hbar \cdot\left(m \cdot \omega_{1}+n \cdot \omega_{2}\right)
$$

В силу сохранения энергии в системе общая мощность равна нулю:

$$
\sum_{m, n} P_{m n}=\sum_{m, n} \hbar A_{m n} \cdot\left(m \cdot \omega_{1}+n \cdot \omega_{2}\right)=0 .
$$

Поскольку $\omega_{2} / \omega_{1}$ иррационально, а $m, n, A_{m n}$ - целые числа, то это равенство выполняется, только если оба слагаемых равны нулю:

$$
\sum_{m, n} m \cdot A_{m n}=\sum_{m, n} n \cdot A_{m n}=0 .
$$

Выразив $A_{m n}$ из (25.1) и подставив в последнее выражение, получим:

$$
\sum_{m, n} \frac{m \cdot P_{m n}}{m \cdot \omega_{1}+n \cdot \omega_{2}}=\sum_{m, n} \frac{n \cdot P_{m n}}{m \cdot \omega_{1}+n \cdot \omega_{2}} .
$$

Рассмотрим соотношения Мэнли-Роу в частном случае трёхчастотного взаимодействия. Пусть, например, комбинационной является разностная частота $\omega_{0}=\omega_{1}-\omega_{2}$. Тогда система имеет три частоты:

$$
\omega_{0}(m=1, n=-1) ; \quad \omega_{1}(m=1, n=0) ; \quad \omega_{2}(m=0, n=1) .
$$

При этом соотношения Мэнли-Роу имеют вид: $P_{0,1} / \omega_{2}=P_{1,-1} / \omega_{0}=P_{1,0} / \omega_{1}$.

Они описывают нелинейную среду с тремя взаимосвязанными резонансами. Если возбудить один из них, то будут возбуждены и остальные. Энергия, запасенная в каждом из резонансов, пропорциональна резонансной частоте, что квантовая механика интерпретирует, как наличие в среде энергетических уровней, пропорциональных частоте. А перекачка энергии между резонансами, обеспечиваемая нелинейностью среды, интерпретиру- 
ется, как перепрыгивание с одного энергетического уровня на другой. Такая схоластическая схема скрывает истинный физический смысл процесса.

Первый, так называемый, квантовый генератор, в котором электромагнитные колебания СВЧ генерировались при помощи молекул $\mathrm{NH}_{3}$, был создан в 1954 Н.Г. Басовым и А.М. Прохоровым и независимо от них Ч. Таунсом (Ch. Townes), Дж. Гордоном (J. Gordon) и Х. Цайгером (H. Zeiger). Оба варианта генераторов работали на пучке молекул аммиака. Его работа так объяснялась с точки зрения квантовой механики: молекулы $\mathrm{NH}_{3}$, обладающие электрическим дипольным моментом, пролетая через неоднородное электрическое поле, по-разному отклоняются этим полем в зависимости от их внутренней энергии. В первом молекулярном генераторе сортирующая система представляла собой квадрупольный конденсатор, состоящий из 4 параллельных стержней, соединённых попарно через один с высоковольтным выпрямителем. Электрическое поле такого конденсатора неоднородно, оно вызывает искривление траекторий молекул, летящих вдоль его продольной оси. Молекулы, находящиеся в верхнем энергетическом состоянии, отклоняются к оси конденсатора и попадают внутрь объёмного резонатора, находящиеся в нижнем - отбрасываются в стороны.

Попадая внутрь резонатора, возбуждённые молекулы испускают фотоны под воздействием поля резонатора. Энергия фотонов усиливает поле в резонаторе, увеличивая вероятность вынужденного испускания для молекул, пролетающих позже (обратная связь). Если вероятность вынужденного испускания фотона больше вероятности поглощения в стенках резонатора и излучения за его пределы, то интенсивность поля резонатора на частоте перехода быстро возрастает за счёт внутренней энергии молекул. Возрастание прекращается, когда поле в резонаторе достигает величины, при которой вероятность вынужденного испускания становится столь большой, что за время пролёта резонатора успевает испустить фотон как раз половина молекул пучка. При этом для пучка в целом вероятность поглощения становится равной вероятности вынужденного испускания (насыщение). Мощность молекулярного генератора на пучке молекул $\mathrm{NH}_{3}$ равна $10^{-11}$ Вт.

Как же на самом деле работает молекулярный генератор на пучке молекул аммиака? У молекулы аммиака действительно есть дипольный момент, и в пространстве эта молекула может иметь два положения: одно устойчивое, а другое - нет. Если при тепловом возбуждении молекула попадает в неустойчивое состояние, то её электрический диполь опрокидывается и начинает колебаться с резонансной частотой. Поскольку размер диполя, определяемый размером молекулы, значительно меньше длины волны излучения, такой резонанс имеет очень большую добротность. Если при помощи квадрупольного конденсатора отфильтровать молекулы в неустойчивом состоянии, а затем вбросить их в макроскопический резонатор, peзонансная частота которого совпадает с частотой колебаний молекулярного диполя, то такие молекулы возбудят в резонаторе колебания, и макроскопический резонатор самостоятельно отберёт молекулы с фазой колебаний, совпадающей с фазой колебаний в нём. Единственное отличие от рубинового лазера - у последнего частота колебаний лежит в области свето- 
вых частот, а у аммиачного - в СВЧ диапазоне. Поэтому принцип работы молекулярного генератора, как и рубинового лазера, укладывается в радиотехнические понятия без привлечения квантовомеханических понятий.

Следует особо подчеркнуть, что в существующих конструкциях лазеров отбор атомов, которые образуют фазированную решетку, формирующую направленный лазерный луч, осуществляет макроскопический резонатор, в котором находится активное вещество. Макроскопический резонатор является сепаратором, отбирающим только соответствующие определённым условиям колебания расположенных в нём атомов. Возбуждённый ударным способом атом, представляющий дипольный излучатель, может находиться в резонаторе в любом месте, и его излучение может иметь любую поляризацию и направление. Но только атомы с частотой, совпадающей с частотой одной из мод макроскопического резонатора, имеют шансы дать вклад в лазерный луч. Для этого нужно, чтобы направление излучения этих атомов совпадало с осью резонатора, и чтобы расстояние от атомов до обеих линз было кратно половине длины волны излучения атома. Тогда излучения атома после многократных отражений от зеркал внешнего резонатора образуют экспонециально убывающую стоячую волну. Если таких атомов много, и возбуждаются они в разное время, они все вместе образуют незатухающую стоячую волну между зеркалами, которая, пройдя через полупрозрачное зеркало, даёт лазерный пучок света. Этот пучок, в силу принципа Гюйгенса, слабо расходится в случае, если размер полупрозрачного зеркала много больше длины волны, что и наблюдается на практике.

Атомы с колебаниями тех атомов, которые не удовлетворяют этим условиям, израсходуют свою колебательную энергию на создание рассеянного некогерентного излучения. Потому-то мы и видим яркое свечение прозрачной трубки газового лазера, через которую такое излучение выходит.

Две конструкции лазера, у которых активное вещество находится за пределами резонатора, представлены на рис. 55.
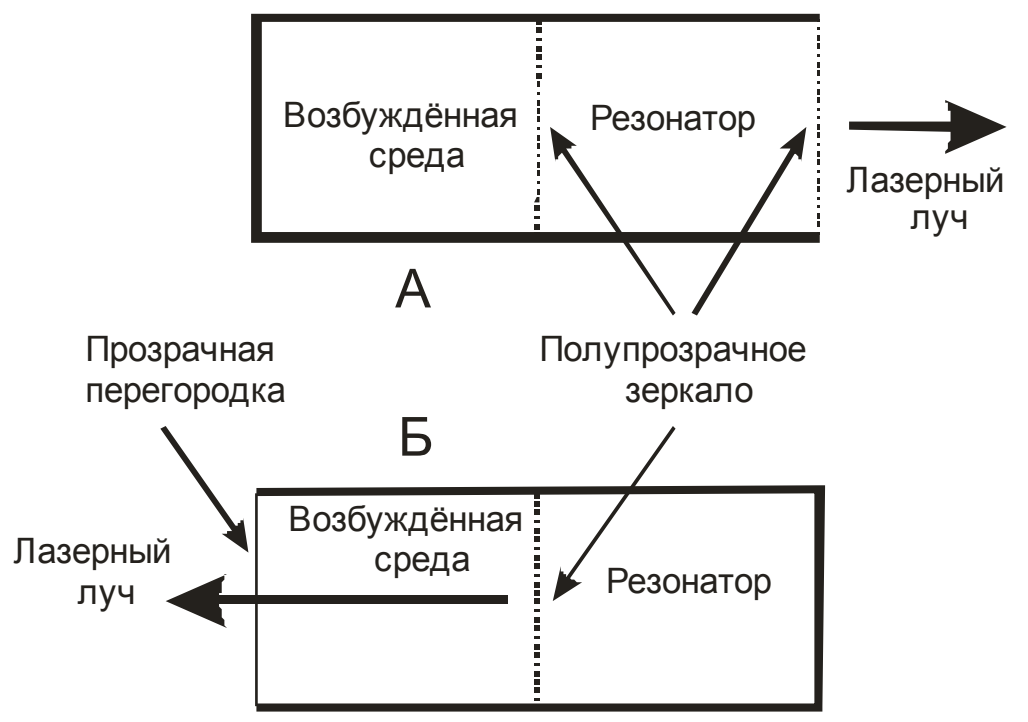

Рис. 55. Схемы лазеров с активным веществом за пределами резонатора 
В варианте А внешний резонатор имеет два полупрозрачных зеркала: через левое зеркало происходит его запитка, а через правое - выходит лазерный луч. Со стороны активного вещества на полупрозрачную стенку резонатора падают волны с самыми различными фазами и поляризацией, но только волны с поляризацией и частотой, совпадающими с одной из мод резонатора, и которые при падении на зеркало окажутся когерентными, возбудят в резонаторе колебания. Эти колебания, пройдя наружу через полупрозрачное зеркало, и образуют лазерный луч. В варианте Б у внешнего резонатора имеется только одно полупрозрачное зеркало, через которое производится и его запитка, и выход луча лазера. Указанные конструкции призваны окончательно разбить миф о том, что лазер является квантовым генератором.

Принцип квантования действия приводит к принципиальным, фактически, неразрешимым проблемам в современной теоретической физике. Из этого принципа получается, что энергия может изменяться только скачкообразно (порциями). Отсюда возникло представление о квантах энергии, которыми обмениваются между собой физические системы. Порочность такого подхода состоит в том, что скачкообразность изменения энергии неизбежно приводит к локальному (на уровне микромира) нарушению закона сохранения энергии даже для консервативных систем. А именно, допускается такое нарушение закона сохранения энергии консервативной системы, при котором величина действия не превышает постоянной Планка. Тем самым, допускается даже бесконечно большое приращение энергии, но только в течение бесконечно малого промежутка времени (чем больше приращение энергии, тем меньше соответствующий временной интервал).

Подобно тому, как в ОТО нарушается закон сохранения энергии для совокупности массивной материи (вещество, антивещество и негравитационные поля) и гравитационного поля, квантование действия тоже приводит к специфическому нарушению закона сохранения энергии. Но это слишком большая потеря для физики, принять которую без самого прямого экспериментального подтверждения представляется недопустимым.

\section{§ 26. Физическое обоснование принципа Гюйгенса и теорема взаимности направленных антенн}

Принцип Гюйгенса гласит, что каждый элемент волнового фронта можно рассматривать, как центр вторичного возмущения, порождающего вторичные сферические волны, а результирующее световое поле в каждой точке пространства определяется интерференцией этих волн. Являясь основным постулатом геометрической оптики, этот принцип не раскрывает физической природы явления. Из геометрической оптики известно, что любой пучок света является расходящимся, и что площадь его сечения увеличивается при распространении. Это явление подчиняется принципу Гюйгенса. Покажем, что физическое объяснение этого принципа существует [69]. 
Рассмотрим плоскую монохроматическую ТЕМ волну, проходящую через щель, ширина которой значительно больше длины волны (рис. 56).

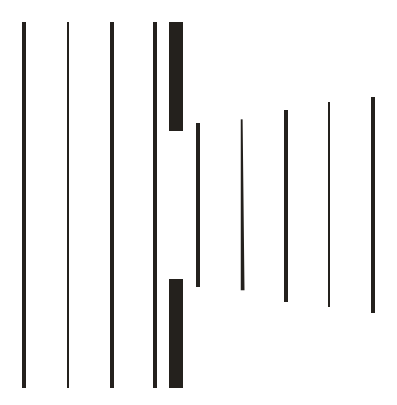

Рис. 56. Прохождение плоской волны через щель

После прохождения щели волна расширяется в поперечном направлении согласно принципу Гюйгенса, когда в расширяющейся волне концы линий постоянной фазы в процессе их движения по направлению распространения волны двигаются со скоростью света ещё и в поперечном направлении. Т.к. при таком расширении увеличивается сечение пучка, то уменьшается вектор Пойнтинга, что означает уменьшение электрического и магнитного поля на линиях постоянной фазы. Это саморасширение электрических векторов на линиях постоянной фазы подобно саморасширению электротоковой волны в длинной линии (см. § 13). Отличием является лишь то, что в линии распространяется волна поперечного электрического поля, и саморасширение происходит в направлении распространения, в данном же случае вектор электрического поля саморасширяется ещё и в поперечном направлении. В длинной линии поперечного расширения нет, т.к. волну в поперечном направлении ограничивают проводники линии. Поперечная трансформация волны сопровождается тем, что, начиная от центра линии постоянной фазы вдоль неё начинает течь ток смещения. Это очень похоже на расширение сжатой резинки, когда все её участки равномерно расширяются. При этом плотность энергии электромагнитной волны уменьшается, равномерно распределяясь в возрастающем объёме расширяющейся волны. Это простое рассмотрение указывает на физические причины постулата Гюйгенса и по сути дела является новым физическим законом.

С этим явлением связана остающаяся без физического обоснования теорема взаимности для антенных систем: коэффициент усиления направленных антенн одинаков как при излучении, так и при приёме сигнала. Странность теоремы в том, что направленная антенна может формировать узконаправленный луч, когда излучаемая энергия концентрируется в каком-то одном направлении. Это значит, что плотность энергии концентрируется в пространстве, ограниченном с боковых сторон. Это хорошо видно на примере лазерного луча. Приёмная же направленная антенна расположена в полях передатчика, которые равномерно распределены в пространстве, и чтобы увеличить свой коэффициент усиления, направленной приёмной антенне нужно собирать энергию с её бокового пространства. Это действительно так, но то, как это она делает, до сих пор остаётся загадкой. 
Известно, что принцип Гюйгенса и вообще законы геометрической оптики работают в случае, когда ширина луча значительно больше длины волны. При ограничении ширины пучка щелью его расходимость растёт, а для щели, меньшей длины волны, получим радиально расходящуюся волну.

При огибании преграды шириной, значительно меньшей длины волны (рис. 57), волны практически её не чувствуют.

$$
|a|
$$

Рис. 57. Огибание волной преграды

Совсем другую картинку мы видим, когда размеры преграды значительно больше длины волны (рис. 58). Если преграда полностью поглощает энергию падающих на неё волн, то волновой процесс выглядит так, как на рис. 58. С правой стороны от поглощающей преграды есть область тени, где волны отсутствуют. Миновав преграду, оборванные концы волн в соответствии с принципом Гюйгенса опять сближаются, принося дополнительную энергию в пространство тени из бокового пространства, находящегося за пределами возможного расположения следующих элементов направленной антенны (явление дифракции). Это характерно для любых волновых процессов, в том числе для электромагнитных волн. Поскольку поглощающая преграда поглотила часть волновой энергии, амплитуда волн справа от тени несколько меньше, чем слева от поглощающего препятствия. Это связано с тем, что отрезки волн справа от препятствия, испытывая дифракцию и расширяясь, переносят свою энергию на участок пространства, занятый тенью. Направление движения энергии в расширяющемся участке волны показано стрелками. Это значит, что для ликвидации тени и восстановления нормального волнового процесса энергия волн перекачивается из боковых участков, отдалённых от области тени.

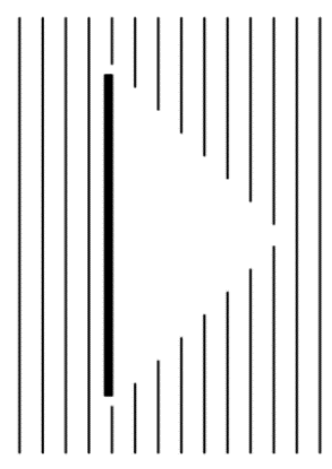

Рис. 58. Случай, когда на пути волн расположена преграда, размеры которой больше длины волны 
Вернёмся к приёмной направленной антенне типа волновой канал и расположим второй директор вне тени в том месте, куда пришли электрические поля, принеся дополнительную энергию из боковых областей. Ситуация в этом случае повторится, опять за таким директором образуется тень. Третий директор мы также можем расположить за пределами тени второго директора и т.д. И каждый раз каждый новый директор будет оказываться в свежих электрических полях, забирая у них положенную ему энергию.

Но ситуация с реальной направленной антенной типа волновой канал другая. Во-первых, длина директора равна половине длины волны, за ним практически нет тени и оборванные куски волны практически сразу смыкаются за директором, но при этом перекачка энергии из боковых участков в область расположения следующего директора всё равно происходит.

Рассмотрим работу такой антенны в поле плоской ТЕМ волны (рис. 59).

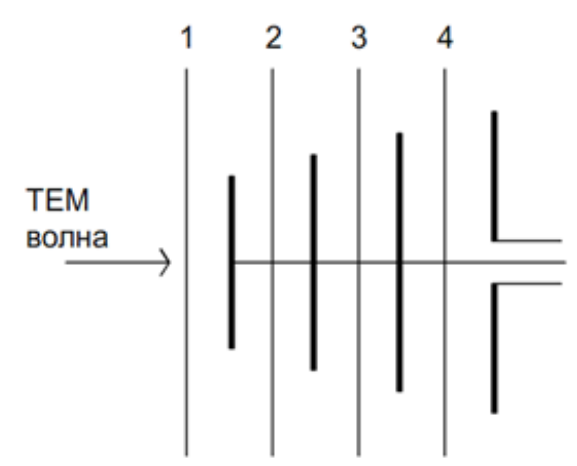

Рис. 59. Антенна типа волновой канал в поле плоской ТЕМ волны

Пусть пронумерованная волна последовательно занимает положения 1,2 , 3 и 4. Долетев до первого директора, она возбуждает в нём токи, делая его излучателем, но при этом теряет часть своей энергии. При этом первый директор переизлучает полученную от волны энергию в окружающее пространство в соответствии со своей диаграммой направленности. Поэтому амплитуда электрического поля волны после прохождения первого директора непосредственно в области за ним меньше, чем до его прохождения.

Для восполнения указанных потерь энергия с боковых участков за пределами антенного поля, начнёт перекачиваться в сторону оси антенны. После прохождения второго директора будет то же самое. Таким образом, дифракция приведёт к тому, что энергия волны с участков за пределами антенного поля начнёт перекачиваться в область нахождения последующих директоров. Токи же, наведённые в директорах, будут делать из них активные вибраторы, которые будут увеличивать токи в каждом последующем директоре и, дойдя до приёмного вибратора, эти поля будут значительно выше поля самой волны в положении 1. С этим и связано то, что направленная приёмная антенна обладает большей эффективностью, чем одиночный вибратор. Хотя, конечно, утверждать, что коэффициенты усиления одинаковы как в режиме передачи, так и в режиме приёма, нельзя. Поэтому теорема взаимности хоть и выполняется, но не в полном объёме. 


\section{$\S 27$. Новая система единиц}

В литературе понятия массы, пространства, времени точно не определены. Масса как физическое понятие обладает следующими фундаментальными свойствами: имеет линейные размеры (иначе она ненаблюдаема), обладает гравитационным и инерционным (для ускорения массы нужно приложить силу) свойствами, а также при определенных условиях может обладать потенциальной и кинетической энергией. Наличие кинетической энергии вытекает из инерционности. Массы притягиваются вследствие наличия вокруг массы потенциального поля, градиент которого даёт силу. Тем самым, система двух удаленных тел имеет потенциальную энергию.

Понятие пространства связано с понятием линейных размеров или длины, пространство трехмерно (для его описания вводятся координатные системы). Другая характеристика пространства, которую можно назвать принципом несовместимости, - в одной и той же точке в данный момент времени не могут находиться две различные массы. Собственно, этот принцип определяет одну из характеристик понятия «время»- разные тела одновременно могут находиться только в разных точках пространства. Что такое время, как и почему оно течет, ученые и философы спорят до сих пор. Время наравне с массой и длиной входит во все системы единиц, как первичная ни от чего не зависящая величина. Однако для измерения времени необходимы часы. Все многочисленные типы часов объединяет одна особенность - взаимодействие других первичных физических величин, таких, например, как массы, длины и силы. Ход маятниковых часов определяется массой Земли и длиной маятника. Это же относится и к спутникам, вращающимся вокруг звезд или планет. В часах с механическими пружинами ход определяется массой и размерами маятника, а также упругими свойствами пружины. В качестве часов могут быть использованы механические резонансные системы, но и здесь в обязательном порядке имеет место взаимодействие трех первичных параметров: силы, массы и длины. Электромагнитные резонансные системы также могут быть использованы в качестве часов, но и здесь их ход будет зависеть от размеров резонатора, а также от диэлектрических и магнитных свойств среды.

Пусть в данной ИСО по каким-то причинам изменилась гравитационная постоянная или инерционные свойства массы, или электродинамические свойства среды - все это повлечет за собой изменение темпа хода часов. Напрашивается вывод о том, что время не является первичной физической величиной, как, например, масса, длина и сила, а непосредственно зависит от этих величин и может быть через них выражена [70, 71].

Немаловажно то, в какую сторону и как быстро течет время. Практически все законы микромира инвариантны по отношению к изменению знака времени, т.е. для них не имеет значения, течет время вперед или назад.

Если система отсчета переходит из одной ИСО в другую, что неизбежно связано с замедлением или ускорением, то в такой системе время должно замедляться или ускоряться. Таким образом, можно считать, что время 
может течь неравномерно, то ускоряясь, то замедляясь. А если так, то может ли время остановиться или поменять направление? Почти очевидно то, что, если бы прекратилось всякое движение, и все тела, включая атомы, замерли на своих местах, то и понятие времени потеряло бы свой смысл. То же самое произошло бы в том случае, если бы Вселенная была абсолютно пустая. Таким образом, напрашивается вывод, что понятие времени является следствием существования материальных объектов и их свойств.

Известно, что обращение (изменение знака) времени, не меняет вида уравнений движения. Это означает, что для любого возможного движения системы может осуществляться обращенное во времени движение, когда система последовательно проходит в обратном порядке состояния, симметричные состояниям, проходимые в предыдущем движении. Естественно предположить, что при отсутствии изменений в системе время для неё вообще не течет. Когда же система после некоторой эволюции возвращается обратным путем в свое исходное состояние, тогда время течет сначала в одном, а затем в другом направлении. Так время обретает смысл собственного времени системы, у каждой - своё. Симметричные по времени состояния отличаются противоположными направлениями скоростей (импульсов) частиц и магнитного поля. Временная инвариантность приводит к определенным соотношениям между вероятностями прямых и обратных реакций, к запрету некоторых состояний поляризации частиц в реакциях, к равенству нулю электрического дипольного момента элементарных частиц и т. д. Из общих принципов квантовой теории поля следует, что все процессы в природе симметричны относительно произведения трех операций: обращения времени, пространственной инверсии и зарядового сопряжения.

Существующие системы единиц не предусматривают разных знаков времени, скорее всего, потому, что время как физическая величина введено не на основе глубоких физических принципов, а решением палаты мер и весов взяты природные периодические процессы, часто разной природы.

Итак, часы обязательно оперируют с другими физическими величинами, например, массой, длиной и силой. Выражается через эти параметры непосредственно квадрат времени, так что само время может принимать значения обоих знаков. Но, хотя масса, длина и сила есть первичные объективно существующие физические величины, трудность состоит в том, что в существующих системах единиц сила выражается через уже введенное время. Однако есть путь преодоления этой трудности. В качестве фундаментальных основ для введения времени, как физической величины, могут быть взяты закон всемирного тяготения, делающий массу носителем силы, и принцип эквивалентности тяжелой (гравитационной) и инертной массы, экспериментально подтверждаемый с очень высокой степенью точности.

Сила притяжения двух одинаковых масс $m$ на расстоянии $2 r$ равна:

$$
F_{g}=m^{2} /\left(4 r^{2}\right) \text {. }
$$

Ниже покажем, какие нужно использовать переводные коэффициенты, чтобы перейти к привычным нам единицам времени.

Если указанные массы вращаются вокруг общего центра масс, то имеем 


$$
T=4 \pi \sqrt{r^{3} / m},
$$

где $T$ - период обращения масс вокруг общего центра.

Соотношение (27.1) включает в себя сразу закон всемирного тяготения и принцип эквивалентности. Оно также определяет размерность времени. Конечно, такая размерность нам несколько непривычна, но привыкли же мы к другим размерностям в физике, в которые входит непонятно откуда взятая секунда. Преимуществом такого подхода является то, что время как физическая величина введено на основе фундаментальных законов физики и, как следствие этого, соответствует принципу обращения времени.

Если единицами длины и массы выбрать метр и килограмм, то в силу (27.1) единицей времени будет $4 \pi$. Для перевода этой величины в секунды разделим её на корень квадратный из гравитационной постоянной. Вновь введенная единица времени оказывается примерно на пять порядков больше секунды. Для избежания этого неудобства можно ввести безразмерный коэффициент, равный корню квадратному из абсолютной величины гравитационной постоянной. При этом соотношения между значениями всех физических величин сохранятся, хотя размерности будут другими. Все механические величины будут выражаться только через длину и массу.

Для перехода к новым электрическим системам единиц нужно в старые вставить новую размерность времени с выбранным безразмерным переходным коэффициентом. Если в Гауссовой системе электрических единиц выразить время в единицах массы и длины, то все электрические и магнитные единицы выразятся в единицах массы и длины.

Следует также отметить, что принятие такого нововведения может привести к серьезной перестройке наших физических взглядов.

\section{§ 28. Капельная модель электрона и атома}

Построим на принципах капельной модели ядра, предложенной Бором и Вайцзеккером [72,73], капельную модель электрона, существующего как в виде шарообразного образования, так и в виде жидкости $[74,75]$.

Явление электризации диэлектриков известно давно. При трении диэлектрики приобретают дополнительный заряд за счёт перехода электронов к диэлектрику с более высокой диэлектрической проницаемостью. Милликен установил, что при распылении в воздухе масла его капли приобретают дискретные заряды. Это позволило сделать вывод о том, что заряды могут иметь только дискретную величину и измеренная дискретная величина заряда капель была определена, как заряд электрона. Опыт не позволяет установить причину дискретности зарядов капель. Эти заряды могли быть получены при превращении масла в капли в процессе его распыления.

Дискретный заряд капли могли получить также при взаимодействии с соплом распыляющего устройства или с атмосферным воздухом. Поскольку диэлектрическая проницаемость воды велика, молекулы атмосферного пара могли забирать заряды у капель масла. В результате этих экспериментов электрон стали считать шарообразным образованием с определёнными 
размерами и дискретным зарядом. Как ответ на вопрос об особенностях его нахождения в составе атома, родилась идея боровских орбит вращения вокруг положительно заряженного ядра. Переход между орбитами сопровождается испусканием квантов электромагнитного излучения, несущих определённую порцию энергии. Эти предположения легли в основу квантовой механики. Но в такой модели до сих пор остаются противоречия. При своём движении электрон должен непрерывно излучать электромагнитные волны, но, двигаясь в составе атома, он не излучает. Кроме этого, простейший атом водорода, состоящий из протона и вращающегося вокруг него электрона, должен иметь магнитный момент, но он его не имеет.

Не ясна природа строения и протона, а также сложных ядер с ядерными силами. Капельную модель строения ядра предложил в 1936 Н. Бор для объяснения больших времён жизни возбужденных ядер тяжёлых элементов, образующихся при захвате медленных нейтронов [72]. Её развил Вайцзеккер, рассматривая ядро, как сферическую каплю несжимаемой заряженной ядерной жидкости [73]. Модель имела большой успех, и с её помощью удалось объяснить многие свойства ядер и, в первую очередь, получить полуэмпирическую формулу для энергии связи ядра.

Электрон может находиться в связанном состоянии в составе атомов, а также в свободном состоянии в виде электронных пучков или вблизи раскалённого катода в электронных приборах. В свободном состоянии электрон находится также в проводниках, свободно перемещаясь в их теле. Но если считать электрон шарообразным образованием определённых размеров, то возникают проблемы. В сверхпроводящем состоянии глубина проникновения магнитных полей и токов составляет величины порядка нескольких сот ангстрем, в то время как величина шероховатости поверхности измеряется микронами. Скорость электронов в сверхпроводящем ниобии при критическом магнитном поле составляет около 300 м/с. Если бы электрон был шариком, то двигаясь по столь извилистой траектории, он за счёт инерционных сил разрушал бы поверхность, но этого не происходит. Поэтому можно предположить, что, находясь в составе проводников, электроны представляют жидкость и двигаются по её законам. Когда же проводник нагревают до высокой температуры, эта жидкость подобно водяному пару испаряется с поверхности проводника. За пределами проводника пары этой жидкости конденсируются в капли, образуя электроны.

Шарообразная форма капли обусловлена поверхностным натяжением, создающим внутреннее давление в капле

$$
p_{\sigma}=2 \sigma / r
$$

где $\sigma$ - коэффициент поверхностного натяжения, $r$ - радиус капли.

Внешнее электрическое поле электрона пытается его разорвать силами, направленными обратно силам поверхностного натяжения. Их давление на поверхность электрона

$$
p_{E}=\varepsilon_{0} E_{s}^{2} / 2
$$

зависит от напряжённости электрических полей на поверхности электрона, 


$$
E_{s}=e^{2} /\left(32 \pi^{2} \varepsilon_{0} r_{e}^{3}\right),
$$

где $e, r_{e}$ - заряд и радиус электрона, $\varepsilon_{0}$ - электрическая постоянная.

Приравнивая (28.1) и (28.2) с учётом (28.3), получаем коэффициент поверхностного натяжения для электронной жидкости

$$
\sigma_{e}=e^{2} /\left(64 \pi^{2} \varepsilon_{0} r_{e}^{3}\right)
$$

Подставляя в (28.4) табличные данные, получаем: $\sigma_{e}=1.5 \times 10^{14}$ Дж $/ \mathcal{M}^{2}$.

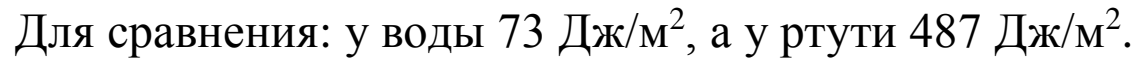

Классический радиус электрона составляет $2.8 \times 10^{-15}$ м. Эксперименты по измерению радиуса протона показали, что его диаметр равен 9х10-16 м. Если разместить протон внутри электрона, то поля протона нейтрализуют заряд электронной жидкости, превратив её в обычную плохо сжимаемую жидкость. Объёмная капля расширится, превращаясь в оболочку (рис. 60), до равновесия между электрическими силами сжатия сферы и силами упругости электронной жидкости. Этот процесс определит радиус атома водорода, который равен 5.3×10 ${ }^{-11}$ м. Поскольку заряд электронной жидкости равен заряду протона, электрические поля снаружи атома отсутствуют.

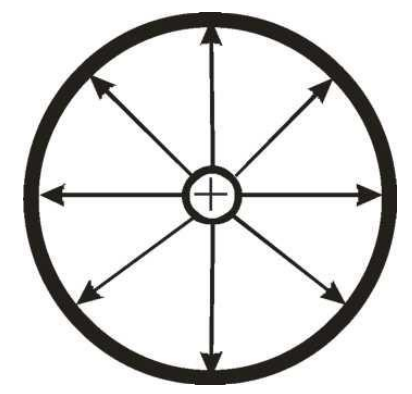

Рис. 60. Капельная модель атома

Распространённой теорией проводимости нормальных металлов является модель Друде. Электроны в металле образуют электронный газ, описываемый кинетической теорией газов. Считается, что электроны, как и атомы газа в кинетической теории, представляют собой одинаковые твердые сферы. Друде предположил, что обеспечивающий электронейтральность положительный заряд принадлежит ионам, которые он считал неподвижными. Несмотря на то, что плотность газа электронов проводимости примерно в 1000 раз больше плотности классического газа при нормальных температуре и давлении, в модели Друде применяются методы кинетической теории нейтральных разреженных газов. Основные предположения теории Друде заключаются в следующем. В интервале между столкновениями не учитывается взаимодействие электрона с другими электронами и ионами и считается, что каждый электрон движется с постоянной скоростью по прямой линии. В присутствии внешних полей электрон движется по законам Ньютона. В модели Друде, как и в кинетической теории, столкновения мгновенны и внезапно меняют скорость электрона, а время между двумя 
очередными столкновениями $\tau$ называется временем релаксации. Это время входит в соотношение, определяющее проводимость металла

$$
\sigma=n e^{2} \tau / m
$$

Плотность тока в металле пропорциональна напряжённости электрического поля: $\mathbf{j}=\sigma \mathbf{E}$. Предполагается, что электроны приходят в состояние теплового равновесия с решеткой исключительно благодаря столкновениям.

Теория Друде удовлетворительно описывает проводимость металлов и до настоящего времени с успехом используется в электродинамике.

Капельная теория, когда электронная компонента в металле рассматривается как электронная жидкость, меняет подход к определению проводимости металла. Задача превращается в гидродинамическую задачу по обтеканию препятствий движущейся жидкостью. Имеется два режима такого обтекания: ламинарный и турбулентный. Для каждого вида течения существует критическое число Рейнольдса $\mathrm{Re}_{c r}$, определяющее переход от ламинарного течения к турбулентному. При $\operatorname{Re} \leq \operatorname{Re}_{c r}$ течение ламинарно, $\mathrm{a}$ при $\operatorname{Re} \geq \operatorname{Re}_{c r}$ возникают турбулентности. При ламинарном течении жидкости энергетические потери отсутствуют, а, следовательно, отсутствует и сопротивление. В турбулентном режиме при огибании препятствий в жидкости возникают турбулентности, приводящие к энергетическим потерям. Именно этим можно объяснить остаточное сопротивление металлов даже при температурах, стремящихся к абсолютному нулю. Колебательные или другие движения обтекаемых препятствий вызывают дополнительные турбулентности, и, следовательно, увеличение сопротивления. И чем больше амплитуда колебаний обтекаемых препятствий, тем больше сопротивление. Это приводит к зависимости сопротивления металлов от температуры. Рассмотренный подход может быть использован для объяснения сверхпроводимости, которая может быть следствием перехода течения электронной жидкости от турбулентного к ламинарному.

Сверхпроводники имеют критическую температуру, ниже которой они переходят в сверхпроводящее состояние. Это означает, что при амплитудах колебаний ионов решетки сверхпроводника ниже некоторого критического значения возможно ламинарное течение электронной жидкости. У сверхпроводников второго рода существует фаза смешанного состояния, когда вихревые образования могут быть созданы наложением внешнего магнитного поля с образованием вихрей Абрикосова. При течении электронной жидкости эти вихри начинают двигаться, что приводит к возникновению сопротивления. Показателен случай, когда в связи с наличием дефектов кристаллической решетки вихри закрепляются на таких дефектах. В этом случае вихри двигаться не могут, и сопротивление отсутствует. В обычной гидродинамике такая ситуация реализована быть не может.

Предложенная модель атома делает задачу нахождения резонансов в нём механической. Число механических резонансов упругой оболочки бесконечно. Они могут носить осевой характер, когда стоячая волна имеет осевую симметрию. Возможны также резонансы, когда вдоль экватора сферы 
укладывается целое число полуволн. Есть и ещё один тип колебаний, порождающих поляризованное по кругу электромагнитное излучение. При столкновении с другими атомами электронная оболочка смещается к ядру с образованием колеблющегося и одновременно вращающегося электрического диполя. Его излучение модулировано по амплитуде, следовательно, спектр излучения атома содержит несущую частоту и боковые частоты.

Гипотеза капельной модели электрона и атома правомерна, как капельная модель ядра. Описана сама идея капельного подхода к описанию электрона, дальнейшее же развитие этих идей в дополнение к капельной модели ядра может привести к созданию обобщённой капельной модели атома.

\section{§ 29. Гравитационный дефект масс}

Пусть пробное тело с массой $m$ падает на очень массивное тело с массой $M \square m$, радиусом $R$ и плотностью $\rho$ (далее - тело $m$ и тело $M$ ), причём в начальный момент времени расстояние между телами очень велико. Скорость падения тела $m$ на поверхность тела $M$ равна:

$$
v=\sqrt{2 \gamma M / R}=2 R \sqrt{2 \pi \gamma \rho / R},
$$

где $\gamma$ - гравитационная постоянная.

Кинетическая энергия падающего тела, полученная от гравитационного поля тела $M$, при падении на поверхность массивного тела превращается в тепловую энергию и излучается в окружающее пространство в виде электромагнитных волн. В итоге, конечная суммарная масса двух тел не равна сумме масс тел до падения (налицо гравитационный дефект масс [76]):

$$
M_{\Sigma} \neq M+m \text {. }
$$

Учитывая то, что при падении тела в гравитационном поле ускорение этого тела не зависит от его массы и (29.1) верно даже для релятивистских скоростей, кинетическая энергия тела $m$ при падении на тело $M$ равна:

$$
E_{k}=m c^{2}\left(\left(1-v^{2} / c^{2}\right)^{-1 / 2}-1\right) .
$$

Теперь нетрудно вычислить гравитационный дефект масс

$$
\Delta m=E_{k} / c^{2}=m\left(\left(1-v^{2} / c^{2}\right)^{-1 / 2}-1\right) .
$$

При падении на земную поверхность этот эффект составляет $\sim m \times 10^{-9}$.

Если $\Delta m<m$, то при падении тела суммарная масса увеличивается. Если же $\Delta m=m$, то рост суммарной массы прекращается, и массивное тело становится идеальной наковальней, превращающей всю массу падающего тела в энергию электромагнитного излучения.

Скорость этого падения (назовём её критической) меньше $c$ :

$$
v_{\kappa p}=c \sqrt{3} / 2,
$$

Из (29.1), (29.2) получаем критический радиус тела $M$, при достижении которого дальнейший рост его массы становится невозможным, 


$$
R_{\kappa p}=3 c /(4 \sqrt{2 \pi \gamma \rho}) .
$$

Возможно ли это для космических объектов? Плотность нейтронных звезд (пульсаров) очень высока [77]. Так, радиус пульсара с массой Солнца $\left(\sim 2 \times 10^{30}\right.$ кг) около 10 км, а его плотность $\sim 5 \times 10^{17}$ кг $/ \mathrm{M}^{3}$. При такой плотности критический радиус около 15 км, а масса $~ 3.4$ масс Солнца. При достижении таких размеров и такой массы нейтронная звезда больше не может увеличивать ни своих размеров, ни свою массу, т.к. любые падающие на неё объекты будут полностью превращаться в энергию.

По предварительным подсчетам в нашей галактике насчитывается около 300 тысяч нейтронных звезд [77]. При столкновении двух нейтронных звёзд произойдет полная аннигиляция нейтронного вещества и превращение его в энергию. Для нейтронной звезды с критическим радиусом 15 км и массой $\sim 3.4$ масс Солнца получаем энергию $5 \times 10^{47}$ Дж, очень близкую к энергии взрыва в ядре галактики NGC 3034, сопровождающегося выбросом из ядра материи массой $6 \times 10^{7}$ масс Солнца. Это явление не находит пока своего объяснения, т.к. не известны источники энергии, способные привести к столь грандиозному взрыву. Рассмотренный процесс столкновения нейтронных звезд может являться именно таким источником.

По сути, такой взрыв - это взрыв ядерного заряда очень большой мощности с разогревом и превращением в плазму больших количеств окружающей материи. Электрические поля при таком взрыве подобны полям при взрыве ядерной бомбы, но гораздо значительнее. Они должны давать специфические поляризационные эффекты (поляризация в электрических полях атомов и молекул, возникновение электрических диполей), что ведёт к поляризации электромагнитных волн, распространяющихся в плазме.

\section{§ 30. Пространственная когерентизация}

При помощи фазированных антенных решеток (ФАР) можно создавать направленные пучки электромагнитных волн сложением (интерференцией) в пространстве пространственно-когерентных волн так, чтобы в определённых направлениях фазы волн от отдельных излучателей складывались, а в других - вычитались. Это единственный путь создания узконаправленных пучков излучения независимо от природы волновых процессов и длины волн. И наоборот, если есть узкий направленный луч, например, лазера, то он образован отдельными излучателями сигналов с большой длиной когерентности, фазы которых в пространстве складываются в тех местах, где есть луч. Но такой луч можно получить и более простым путём. Если солнечный свет пропустить через отверстие, то он образует почти прямолинейный луч. Но ведь само Солнце испускает не монохроматические и далеко не когерентные волны. В чём же тогда дело? Если сделать хорошую голограмму при помощи лазера, испускающего, например, красный луч, и осветить её солнечным светом, то можно увидеть голографическую картинку в красном свете. Этот эксперимент ещё раз подтверждает, что в со- 
ставе солнечного света имеется данная спектральная линия с большой длиной когерентности, поскольку только при помощи когерентного света можно увидеть голограмму. Поверхность Солнца испускает совсем не когерентное излучение, однако на больших расстояниях от Солнца оно становится когерентным. Но раз имеется узконаправленный луч, значит, он образован сложением когерентных волн. Если при помощи отверстия вырезать луч от далёкой звезды, то он будет обладать ещё меньшей расходимостью и большей когерентностью, чем солнечный. Тот же опыт можно проделать и с далёкой лампочкой, которая является практически точечным излучателем, и получить от неё направленный луч. Итак, чем меньше телесный угол, под которым виден источник, тем большую когерентность сигнала он даёт, причём во всём диапазоне излучаемого им спектра. Если из этого сигнала при помощи фильтра выделить определённую узкую полосу спектра, то такой луч ничем не будет отличаться от лазерного.

Но в существующей литературе нет объяснения такой странной особенности точечных источников света, хотя суть дела проста. Узконаправленный луч в данной точке пространства может быть образован только сложением когерентных составляющих от отдельных излучателей. На Солнце или звезде каждый атом имеет свою диаграмму направленности дипольного излучателя. Будучи возбуждённым тепловыми ударами, такой атом определённое время излучает в пространство свой спектр колебаний до очередного столкновения с другим атомом. Поскольку таких атомов очень много, хоть фазы их излучения и хаотичны, но всегда найдётся определённое количество атомов, фазы излучения которых в каком-то удалённом месте совпадут, где мы и видим результат этого сложения в виде прямолинейного луча. Поэтому любой точечный источник - это своего рода лазер, излучающий сферический полихроматический луч. И уже из этого луча можно при помощи отверстий получать узкие радиальные лучи, а при помощи фильтров делать их монохроматическими.

\section{§ 31. Ферроэлектрический трансформатор}

Симметричность законов магнитоэлектрической и электромагнитной индукции ведёт к симметрии технических решений. Например, для создания электродвигателей используют вращающееся поле, причём как магнитное, так и электрическое. В технике широко используются трансформаторы с ферромагнитными сердечниками, при помощи магнитного потока передающие энергию из одной обмотки в другую. Их большой недостаток - неработоспособность на высоких частотах в связи с большой инерционностью перемагничивания сердечника. Симметрия законов подсказывает существование трансформаторов с сердечником не из ферромагнетика, а из ферроэлектрика [78], работоспособных на очень высоких частотах в связи с очень малой инерцией электрической поляризации (рис. 61, 62).

В состав трансформатора на рис. 61 входит плоский конденсатор, между пластинами которого размещён цилиндр из ферроэлектрика с большой ди- 
электрической проницаемостью. На цилиндре размещена обмотка тора, концы которой подключены к клеммам 2. При подаче на конденсатор переменного напряжения в цилиндре будут течь поляризационные токи, и вокруг цилиндра возникнет переменная во времени циркуляция магнитного поля, возбуждающая в торообразной обмотке токи, и на клеммах 2 появится переменная разность потенциалов.

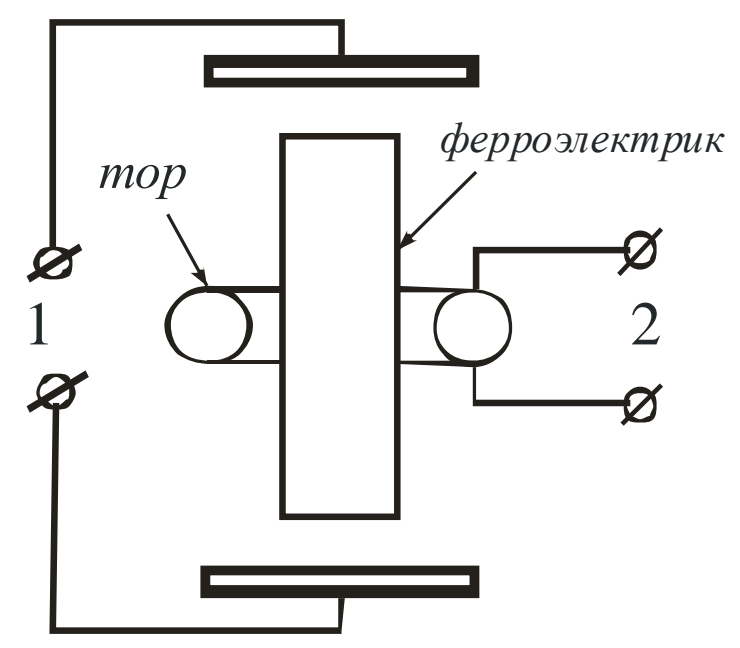

Рис. 61. Схема ферроэлектрического трансформатора

Трансформатор на рис. 62 образован торобразным сердечником из ферроэлектрика с двумя торобразными обмотками. Коэффициент трансформации зависит от соотношения числа витков в обмотках. При подключении к обмоткам конденсаторов он становится резонансным трансформатором.

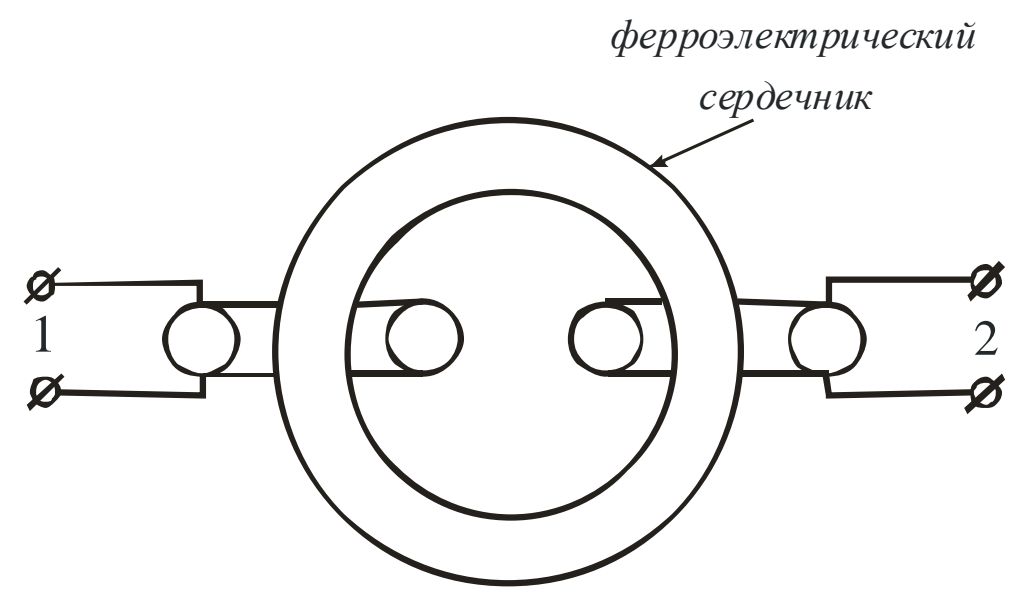

Рис. 62. Трансформатор с торобразным ферроэлектрическим сердечником

Несмотря на простоту идеи и конструкции, такие трансформаторы до работы [78] не были описаны. Они весьма перспективны. Магнитные усилители обладают высокой надёжностью, но широко не применяются, т.к. работают на низких частотах. С рассмотренным трансформатором можно создать надёжные широкополосные (до очень высоких частот) усилители. 


\section{ЗАКЛЮЧЕНИЕ}

В результате проведённых исследований установлена зависимость электрического поля заряда от параметров его движения. В основе электродинамики должны лежать три закона: закон Кулона, закон зависимости электрического поля заряда от скорости его движения и закон зависимости электрического поля заряда от его ускорения. Закон Кулона определяет электрическое поле неподвижного заряда. Наличие у заряда скорости приводит к приращению его электрического поля в поперечном относительно скорости направлении. А ускорение заряда приводит к индуцированию дополнительного электрического поля в продольном относительно ускорения направлении. Закон Кулона и закон зависимости электрического поля заряда от его ускорения уже содержатся в электродинамике Максвелла.

Принципиально новым же является закон зависимости электрического поля заряда от скорости его движения. Объединение всех этих трёх законов в единый фундамент электродинамики коренным образом меняет её идеологию. Оно позволяет рассматривать с единых позиций и статическое взаимодействие зарядов, и законы силового взаимодействия в случае их взаимного движения, и законы излучения и рассеивания. Такой подход позволил преодолеть описанные в данной монографии теоретические проблемы электродинамики, объяснить без привлечения СТО фазовую аберрацию и поперечный эффект Доплера, что не удавалось сделать в рамках традиционной идеологии электродинамики.

Важнейший результат работы - установление зависимости скалярного потенциала электрического заряда от скорости его относительного движения (концепция скалярно-векторного потенциала) и обоснование этой зависимости в рамках лагранжева формализма. Принятие данной концепции ответственно, но физически прозрачно, ведь фактор движения заряда при учете его влияния на окружающие заряды может отражаться на его энергетических характеристиках, так что рост кинетической энергии при ускорении заряда связан с изменением его электрических полей.

Экспериментальным подтверждениям концепции уделено в работе значительное место. Одно из них - возникновение импульса электрического поля при мощном взрыве, так что космические ядерные взрывы открыли в начале 60 -х годов новую страницу даже в фундаментальной физике.

Уравнения Максвелла, приводящие к волновым уравнениям для электромагнитных полей, и соотношения для силового взаимодействия токонесущих систем с постулируемой силой Лоренца лежат в основе двух фактически независимых разделов классической электродинамики. Предложенная концепция соединяет их на единой идейной основе.

Получены преобразования полей при переходе из одной ИСО в другую, с точностью до членов $\sim v^{2} / c^{2}$ совпадающие с релятивистскими. Все экспериментальные данные подтверждают СТО не точнее указанного порядка.

Показано, что наряду с параметрами $\varepsilon \varepsilon_{0}$ и $\mu \mu_{0}$, характеризующими накопленные или переносимые в среде электрическую и магнитную энер- 
гии, существуют еще два фундаментальных материальных параметра, аналогично характеризующие кинетическую и потенциальную энергии: кинетическая индуктивность $L_{k}$ и кинетическая ёмкость $C_{k}$. Но только кинетическая индуктивность использовалась при описании некоторых физических явлений, например, в сверхпроводниках. Использование всех четырех параметров проясняет физическую картину волновых и резонансных процессов в линейных средах при наличии в них электромагнитных полей и позволяет решать для них задачи электродинамики. Единственными переносчиками энергии в материальных средах считались электромагнитные волны, что не учитывает всех видов энергии, накапливаемой и переносимой в них полями и токами. Новый подход вводит в рассмотрение магнитоэлектрокинетические и электромагнитопотенциальные волны, в которых часть энергии накапливается и переносится чисто механическим путем. Резонансы в материальных средах тоже носят свою специфику. В отличие от электромагнитных резонансов в замкнутых плоскостях, когда обмен энергией происходит между магнитными и электрическими полями, в материальных средах есть два вида резонансов. Первый - электрокинетический, когда энергия электрического поля превращается в кинетическую энергию носителей зарядов и наоборот, а магнитных полей нет вообще. Второй - магнитнопотенциальный, когда потенциальная энергия, накопленная в прецессионном движении магнитных моментов, может отдаваться во внешнее пространство на частоте прецессии.

Физическая интерпретация величин в электродинамике требует осторожности, так как не все они являются физическими величинами. Остальные же представляют собой выражающиеся через какие-либо физические величины математические параметры, в качестве примеров которых можно привести диспергирующие (зависящие от частоты) диэлектрическую и магнитную проницаемости среды. Явление дисперсии электромагнитных волн при их прохождении через материальные среды означает зависимость фазовой скорости волны от частоты. В общепринятой интерпретации эта зависимость порождена дисперсией диэлектрической проницаемости среды. Предложена более адекватная новая интерпретация, раскрывающая механизм формирования данной зависимости сразу несколькими независимыми от частоты параметрами. Для плазмы ими являются диэлектрическая проницаемость вакуума и кинетическая индуктивность носителей зарядов, представляющих плазму. В диэлектриках в этом процессе, наряду с диэлектрической проницаемостью вакуума и кинетической индуктивностью связанных зарядов, принимают участие поляризационные или ориентационные свойства электрических диполей, имеющихся в диэлектрике. Следовательно, дисперсия электромагнитных волн, наблюдающаяся при их распространении в диэлектриках, связана с дисперсией проводимости диэлектриков. В формировании этой дисперсии проводимости принимают участие указанные параметры, причем они от частоты не зависят.

Использование хорошо подходящего для случая диэлектриков вектора поляризации в электродинамике проводящих сред на микроскопическом 
уровне ограничивает адекватность соответствующих теоретических моделей, поскольку заряды в проводниках являются свободными и не могут образовывать электрические диполи. Это не позволяло обнаружить новое физическое явление - поперечный плазменный резонанс, который может иметь место в незамагниченной ограниченной плазме наряду с продольным ленгмюровским резонансом, причем частоты у этих резонансов совпадают, т.е. они являются вырожденными. А это означает, что игнорировалось целое научное направление, имеющее важное прикладное значение, т.к. на его основе могут быть созданы лазеры на коллективных колебаниях плазмы, резонансные фильтры. Указанное явление также может быть использовано для разогрева плазмы.

В работах $[9,17,21,22,80,81]$ отмечается ряд несовершенств электродинамики: наличие исключений в законе Фарадея, постулирование силы Лоренца, отсутствие связи между уравнениями Максвелла и описанием взаимодействия токонесущих систем, введение постулатов для получения преобразований полей при переходе из одной ИСО в другую. В физическом плане все эти несовершенства устраняет признание фундаментальности понятия электрического поля и признание зависимости электрического поля заряда от параметров его движения, позволяя на этой основе построить единую непротиворечивую электродинамику. Математически это сопряжено с совершенствованием аппарата дифференциального исчисления полевых функций в направлении возможности оперировать транскоординатными дифференциальными и интегральными уравнениями.

Многие выдающиеся учёные (Ампер, Гроссман, Гаусс, Ленц, Нейман, Вебер, Риман, Николаев, Маринов и др.) подвергали сомнению концепцию электромагнитного поля Фарадея-Максвелла. Предлагаемая в монографии альтернативная идеология электродинамики полностью отвергает эту концепцию. В отличие от электрического поля, магнитное поле не существует, как физическое поле, а является математической абстракцией. Из этого следует, что для полного и непротиворечивого описания электромагнитных явлений следует отказаться и от постулата о силе Лоренца, и от уравнений Максвелла в любой их формулировке, включая оригинальную на языке кватернионов и представление Герца-Хевисайда.

Одной из неотъемлемых составляющих концепции Фарадея-Максвелла является представление об электромагнитных волнах, как особой материальной субстанции, обладающей импульсом подобно материальным частицам. Поэтому знаменитые опыты Лебедева по обнаружению давления света убедили тогда в справедливости концепции Фарадея-Максвелла до этого непримиримых её оппонентов. Но пондеромоторное действие оказывает не только волна, но и постоянное поле. В монографии показано, что пондеромоторное действие электромагнитной волны есть лишь частное проявление более фундаментального явления - силового взаимодействия токонесущих систем, подчиняющегося закону сохранения импульса. Понятие же о давлении электромагнитной волны не имеет определённого смысла. Итак, вслед за отказом от концепции электромагнитного поля следует отказаться и от представления о наличии импульса у ЭМ волн. 
Понятия импульса и кинетической энергии неприменимы к электрическому полю, как таковому, а применимы лишь к материальным частицам и образованным ими материальным телам. Тем самым, существует принципиальная разница между полями и частицами, так что вряд ли можно говорить о корпускулярно-волновом дуализме, как об одном из фундаментальных физических принципов. Лагранжево-гамильтонов формализм в теории поля основан на представлении поля в виде механической системы с бесконечным числом степеней свободы. Но если электрическое поле не несёт импульса, то такое его представление в виде механической системы лишено физического смысла, а ведь оно лежит в основе квантовой теории поля. Более того, предлагаемая в монографии альтернативная идеология электродинамики позволяет раскрывать неквантовые механизмы физических явлений и технических процессов, традиционно считающиеся квантовыми. Так, показано, что работа квантовых генераторов (лазеров) вполне укладывается в рамках радиотехнических моделей, если представлять их фазированными антенными решётками с микроскопическими излучателями.

Главный итог данной работы - обоснование давно назревавшей необходимости коренных преобразований не только в классической электродинамике с её математическим аппаратом, но и в физике в целом (включая механику и физику микромира) вместе с математической физикой.

Зависимость электрического поля заряда от скорости не является очередным физическим постулатом, а имеет более фундаментальную природу, далеко выходящую за пределы собственно электродинамики. Фундаментальные физические причины такой зависимости указывают на необходимость построения новой физики, основанной на новых представлениях о пространстве и времени. В соответствии с ними пространство и время не являются самостоятельными, независимыми формами, как в классической нерелятивистской физике, а соединены друг с другом в единое пространство-время. Но, оказывается, что пространство и время соединены не преобразованиями Лоренца в единый континуум, а «старыми добрыми» преобразованиями Галилея в единый гиперконтинуум. Такие представления о пространстве и времени можно считать неоклассическими в том смысле, что они возвращают преобразованиям Галилея их фундаментальную роль.

В основе гиперконтинуальной физики лежат четыре динамических закона сохранения: три заимствованных из классической нерелятивистской физики (закон сохранения энергии, закон сохранения импульса, закон сохранения момента импульса) и новый - закон сохранения кинетического баланса, заменяющий закон сохранения 4-импульса из теории относительности. Все четыре динамических закона сохранения вытекают из вариационного принципа наименьшего действия в предположении надлежащих свойств пространства и времени, соединенных в единый пространственновременной гиперконтинуум. Так же, как и в классической нерелятивистской физике, из однородности времени (инвариантность лагранжиана относительно временных сдвигов) следует закон сохранения энергии, из однородности пространства (инвариантность лагранжиана относительно пространственных трансляций) - закон сохранения импульса, из изотропности 
пространства (инвариантность лагранжиана относительно пространственных вращений) - закон сохранения момента импульса. Закон сохранения 4-импульса, который в теории относительности вытекал из Лоренцинвариантности метрики пространства-времени (инвариантность лагранжиана относительно преобразований Лоренца) в гиперконтинуальной физике утрачивает свою силу, уступая место новому закону сохранения кинетического баланса, который вытекает из изотропности гиперконтинуума (инвариантность лагранжиана относительно изовелоцитарных, то есть сохраняющих модуль вектора скорости, преобразований Галилея).

Одним из следствий закона сохранения кинетического баланса является как раз зависимость скалярного потенциала заряда от скорости. Из этого же закона вытекает и зависимость от скорости ряда других величин, в частности, массы. В СТО накопление движущимся телом кинетической энергии связывается с увеличением его массы, но не с изменением параметров создаваемых им физических полей, отвечающих за это накопление. В гиперконтинуальной же физике ускорение тела связано с относительными изменениями всех таких полей, включая гравитационное, динамика которого также отлична от статики.

Итак, оказывается возможным механическое движение со сверхсветовой скоростью, раскрывается существенная специфика вращательного движения, открываются перспективы преодоления ряда проблем физики микромира, в частности, локального нарушения динамических законов сохранения. Подобно тому, как релятивистская идеология электродинамики привела к построению релятивистской физики, предлагаемая в данной работе альтернативная идеология электродинамики претендует на роль инициатора процесса создания новой, гиперконтинуальной физики. Что же может помешать внедрению в теорию и практику столь продуктивных идей? Во введении уже обсуждался этот вопрос. Наша задача, задача учёных, довести это дело до конца.

В заключение отметим ещё одно практически важное обстоятельство. Существующая теория возникновения ЭМИ ядерного взрыва, опирающаяся на сложившуюся идеологию электродинамики, не выдерживает критики. Уже были не оправдавшиеся, хотя и полностью обоснованные в рамках этой теории предсказания Нобелевского лауреата Ханса Альбрехта Бете. Новый «обоснованный» ядерный взрыв в космосе погубит всю спутниковую информационную систему, тогда оправдываться будет поздно.

\section{БЛАГОДАРНОСТИ}

Авторы благодарят профессора Анри Амвросьевича Рухадзе и профессора Николая Николаевича Горобца за полезные советы, которые способствовали улучшению монографии, а также Николая Александровича Дробышева и Ивана Фёдоровича Горина за участие в решении отдельных задач, рассматриваемых в монографии. 


\section{СПИСОК ЛИТЕРАТУРЫ}

1. Джемс Клерк Максвелл. Избранные сочинения по теории электрического поля. М.: Государственное издательство технико-теоретической литературы, 1954.

2. П. К. Рашевский. Риманова геометрия и тензорный анализ. М.: Наука, 1967.

3. С. Маринов (Marinov S.) Физическая мысль России 1. 1995. С. 52 - 77.

4. Р. Фейнман, Р. Лейтон, М. Сэндс. Фейнмановские лекции по физике. М: Мир, 1977.

5. F. F. Mende, Problems of Lorentz Force and Its Solution. International Journal of Physics, 2014, Vol. 2, No. 6, p. 211-216.

URL: http://pubs.sciepub.com/ijp/2/6/5

6. М. Фарадей. Экспериментальные исследования по электричеству, в трёх томах. М.: Изд. АН СССР, 1959.

7. М. Фарадей. Избранные работы по электричеству. Москва - Ленинград: Государственное объединённое научно-техническое издательство редакция научно-технической литературы, 1939.

8. Ф. Ф. Менде, А. И. Спицын. Поверхностный импеданс сверхпроводников. Киев: Наукова думка, 1985.

9. Ф. Ф. Менде. Новая электродинамика. Революция в современной физике. Харьков: НТМТ, 2012.

10. Ф. Ф. Менде. К вопросу об уточнении уравнений элетромагнитной индукции. Харьков, депонирована в ВИНИТИ, №774-В88 Деп., 1988, 32с.

11. A. A. Rukhadze, A.F. Alexandrov, L.S. Bogdankevich, Principles of Plasma Electrodynamics, M. URSS, KRASAND 2013, 489 (Second Edition, first Edition, Spriger, Hidelberg, New-York, Tokio 1984).

12. Л. Д. Ландау, Е. М. Лифшиц. Электродинамика сплошных сред. М.: Физматгиз, 1973.

13. В. Л. Гинзбург. Распространение электромагнитных волн в плазме. М.: Наука. 1967.

14. Л. Д. Ландау, Е. М. Лифшиц. Теория поля. М.: Наука, 1988.

15. В. Г. Левич. Курс теоретической физики. М.: Физматгиз, 1962.

16. Л. А. Арцимович. Что каждый физик должен знать о плазме. М.: Атомиздат, 1976.

17. F. F. Mende. Kinetic Induktance Charges and its Role in Classical Electrodynamics. Global Journal of Researches in Engineering, J General Engineering, 2014, Vol. 3, No. 5, p. 51-54.

URL: http://www.engineeringresearch.org/index.php/GJRE/article/view/1260

18. F. F. Mende. On refinement of certain laws of classical electrodynamics. arXiv, physics/0402084.

19. Ф. Ф. Менде. Роль и место кинетической индуктивности зарядов в классической электродинамике. Инженерная физика, 2012, №11, с. 10-19. 20. Ф. Ф. Менде. Новые подходы в современной классической электродинамике. Часть I. Инженерная физика, 2013, №1, с. 35-49.20. Ф. Ф. Менде, 
Кинетическая индуктивность и её роль в электродинамике. LAP LAMBERT Academic Publishing, 2013.

21. F. F. Mende. What is Not Taken into Account and they Did Not Notice Ampere, Faraday, Maxwell, Heaviside and Hertz. AASCIT Journal of Physics, 2015, Vol.1, No. 1, p. 28-52.

URL: http://www.aascit.org/journal/archive2?journalId=977\&paperId=1653 22. Ф. Ф. Менде, Проблемы современной физики и пути их решения. PALMARIUM Academic Publishing, 2010.

23. F. F. Mende. Concept of the dispersion of electric and magnetic inductivities and its physical interpretation. Global Journal of Researches in Engineering: A Mechanical and Mechanics Engineering, 2014, Vol. 14, No. 8, p.11-18. URL: https://globaljournals.org/GJRE_Volume14/3-Concept-of-the-Dispersion. 24. F. F. Mende Conception of the scalar-vector potential in contemporary electrodynamics, arXiv.org/abs/physics/0506083.

25. F. London, Superfluids. Vol.1. Microscopic theory of superconductivity. Nev York: Dower publ., 1950.

26. F. F. Mende. Transversal plasma resonance in a nonmagnetized plasma and possibilities of practical employment of it. arXiv, physics/0506081.

27. F. F. Mende, Transverse Plasma Resonans Mode in an Nonmagnetized Plasma and Its Practical Applications. American Journal of Electrical and Electronic. Engineering, 2014, Vol. 2, No. 5, p. 152-158.

URL: http://pubs.sciepub.com/ajeee/2/5/3

28. А. Ярив. Квантовая электродинамика и нелинейная оптика. М.: Сов. радио, 1973.

29. Ф. Ф. Менде. Кинетическая электрическая ёмкость. Инженерная физика, 2013, №3, с. 49-51.

30. F. F. Mende. Symmetrization and the Modification of the Equations of Induction and Material Equations of Maxwell. AASCIT Journal of Physics, 2015, Vol.1, No. 3, p. 171-179.

URL: http://www.aascit.org/journal/archive2?journalId=977\&paperId=2196

31. В. В. Никольский, Т. И. Никольская. Электродинамика и распространение радиоволн. М.: Наука, 1989.

32. F. F. Mende. Updated Electrodynamics. AASCIT Journal of Physics, 2015, Vol.1, No. 3, p. 149-170.

URL: http://www.aascit.org/journal/archive2?journalId=977\& paperId=2187 33. Ф. Ф. Менде. Существуют ли ошибки в современной физике. Харьков: Константа, 2003.

34. F. F. Mende, New Properties of Reactive Elements and the Problem of Propagation of Electrical Signals in Long Lines. American Journal of Electrical and Electronic Engineering, 2014, Vol. 2, No. 5, p. 141-145. URL: http://pubs.sciepub.com/ajeee/2/5/1

35. F. F. Mende. Induction and Parametric Properties of Radio-Technical Elements and Lines and Property of Charges and Their Flows. AASCIT Journal of Physics, 2015, Vol.1, No. 3, p. 124-134.

URL: http://www.aascit.org/journal/archive2?journalId=977\&paperId=2144 
36. F. F. Mende. New Properties of Reactive Elements, Lines of Transmission of Energy and the Relaxation Properties of Electronic Fluxes and Conductors. AASCIT Journal of Physics, 2015, Vol.1, No. 3, p. 190-200.

URL: http://www.aascit.org/journal/archive2?journalId=977\&paperId=2262 37. 150 лет волн Герца, Академия наук СССР, 1938.

38. Ф. Ф. Менде. Как скорость удлинения длинной линии влияет на её входное сопротивление. Инженерная физика, 2015, №12, с. 3-5.

39. Ф. Ф. Менде. Великие заблуждения и ошибки физиков XIX-XX столетий. Революция в современной физике. Харьков: НТМТ, 2010.

40. Ф. Ф. Менде. Новые подходы в современной классической электродинамике, Часть II. Инженерная физика, 2013, №2, с. 3-17.

41. F. F. Mende, Concept of Scalar-Vector Potential in the Contemporary Electrodynamic, Problem of Homopolar Induction and Its Solution. International Journal of Physics, 2014, Vol. 2, No. 6, p. 202-210.

URL:http://pubs.sciepub.com/ijp/2/6/4

42. F. F. Mende, Consideration and the Refinement of Some Laws and Concepts of Classical Electrodynamics and New Ideas in Modern Electrodynamics. International Journal of Physics, 2014, Vol. 2, No. 8, p. 231-263.

URL: http://pubs.sciepub.com/ijp/2/6/8

43. F. F. Mende. Concept of Scalar-Vector Potential and Its Experimental Confirmation. AASCIT Journal of Physics, 2015, Vol.1, No. 3, p. 135-148.

URL: http://www.aascit.org/journal/archive2?journalId=977\&paperId=2176 44. F. F. Mende. Classical Relativistic Corrections to Coulomb Law. AASCIT Journal of Physics, 2015, Vol.1, No. 2, p. 69-75.

URL: http://www.aascit.org/journal/archive2?journalId=977\&paperId=1924 45. F. F. Mende. The Classical Conversions of Electromagnetic Fields on Their Consequences. AASCIT Journal of Physics, 2015, Vol.1, No. 1, p. 11-18.

URL: http://www.aascit.org/journal/archive2?journalId=977\&paperId=1647

46. В. Босс. Уравнения математической физики. М.: ЛИБРОКОМ, 2009.

47. А. С. Дубровин. От эталонной модели защищенной автоматизированной системы к общей теории пространства-времени. Вестник Воронежского института высоких технологий. 2010. № 7. с. 37-41.

48. А. С. Дубровин, А.В. Скрыпников, Т.В. Лютова, Е.В. Чернышова, Е.В. Глазкова. Создание эталонной модели защищенной автоматизированной системы в контексте смены естественнонаучных парадигм. Современные проблемы науки и образования. 2015. № 1.

URL: www.science-education.ru/121-18620.

49. А.С. Дубровин, С.Ю. Хабибулина. Пространство-время и информатика: от критики континуума до критики принципа геометризации. Фундаментальные исследования. 2014. № 6. Часть 4, с. 714-718.

50. F. F. Mende. Mechanical and Thermal Electrization Metal, Dielectrics and Plasma. International Journal of Modern Physics and Application. 2015. Vol. 2. № 6. p. 73-99.

51. А. С. Дубровин. Транскоординатная электродинамика в пространственно-временном гиперконтинууме. Международный журнал прикладных и фундаментальных исследований, 2015. № 12. с. 34-41. 
52. А. С. Дубровин. Алгебраические свойства функций одномерных синусоидальных волн и пространство-время. Вестник Воронежского государственного университета. Физика. Математика, 2013. № 1. с. 5-19.

53. А. С. Дубровин, А. В. Скрыпников, Т. В. Лютова, Е. В. Глазкова, Е. В. Чернышова. Общенаучные итоги создания эталонной модели защищенной автоматизированной системы. Фундаментальные исследования. 2015. № 2. Часть 15. с. 3247-3251.

54. A. S. Dubrovin Application of the principle of hierarchy in computer science to representations about space-time in the theoretical physics. International Journal of Applied And Fundamental Research. 2014. № 1.

URL: www.science-sd.com/456-24490.

55. А. С. Дубровин. Модели и методы комплексного обеспечения надежности информационных процессов в системах критического применения: дис. докт. техн. наук. Воронеж, 2011. с. 433.

56. Дж. Джексон. Классическая электродинамика. М.: Мир, 1965.

57. К. Шимони. Теоретическая электротехника. М.: Мир, 1964.

58. А. С. Дубровин. Преобразования Менде в транскоординатной электродинамике. Международный журнал прикладных и фундаментальных исследований, 2015. № 12. с. 1006-1012.

59. Ф. Ф. Менде. К вопросу о возникновении вторичных электрических полей при протекании через сверхпроводники постоянных токов. Харьков, 1992. - 28 с. № 3182-В92. Деп.

60. F. F. Mende. Experimental corroboration and theoretical interpretation of dependence of charge value on DC flow velocity through superconductors. Proceedings International Conference Physics in Ukraine, Kiev, 1993.

61. Знакомый и незнакомый Зельдович (в воспоминаниях друзей, коллег, учеников). М: Наука, 1993, (под ред. С. С. Герштейна и Р.А. Сюняева).

62. Б.В. Болт. В глубинах Земли: о чем рассказывают землетрясения. М., 1984.

63. Дж. Гир, Х. Шах. Зыбкая твердь. М., Мир, 1988.

64. Х. Гупта, Б. Растоги. Плотины и землетрясения. М., Мир, 1979.

65. Н. В. Короновский. Общая геология. Изд-во Московского ун-та, 2002.

66. F. F. Mende. Electrodynamics and thermodynamics of nuclear explosions and TNT. LAP LAMBERT Academic Publishing, 2014.

67. Ф. Ф. Менде. Электрополевая спектроскопия. Инженерная физика, № 9, 2012, c. $16-18$.

68. F. F. Mende. Electro Spectroscopy of Materials and Samples. Journal of Materials Sciences and Applications 2015, Vol.1, No. 2, p. 70-77.

69. F. F. Mende, Physical Substantiation of Huygens Principle and the Reciprocity Theorem. American Journal of Electrical and Electronic Engineering, vol. 2, no. 6 (2014): 165-170.

URL: http://pubs.sciepub.com/ajeee/2/6/2

70. F. F. Mende. Material Space Motion Time - New Ideas and the Practical Results, AASCIT Journal of Physics, Vol.1, No. 4, Publication Date: July 7, 2015, Page: 222-228.

URL: http://www.aascit.org/journal/archive2?journalId=977\&paperId=2306 
71. F. F. Mende. Material Space Motion Time Phenomenon of Kinetic Energy and Inertia of Material Bodies, AASCIT Journal of Physics, Vol.1, No. 4, Publication Date: July 13, 2015, Page: 292-296.

URL: http://www.aascit.org/journal/archive2?journalId=977\&paperId=2185

72. Н. Бор. Захват нейтрона и строение ядра. УФН, Том 4, № 4, 1936.

73. К. М. Мухин. Экспериментальная ядерная физика. Москва: Энергоатомиздат, 1993.

74.Ф. Ф. Менде. Капельная модель электрона и атома. Инженерная физика, №3, 2015, c. 15-16.

URL: http://infiz.tgizd.ru/ru/arhiv/13754

75. F. F. Mende. Liquid-Drop Model of Electron and Atom, AASCIT Journal of Physics, Vol.1 , No. 2, Publication Date: May 6, 2015, Page: 107-110

URL: http://www.aascit.org/journal/archive2?journalId=977\&paperId=1787

76. F. F. Mende, Gravitational mass defect. International Journal of Physics. 2015, Vol. 3, No. 1, 29-31.

URL: http://pubs.sciepub.com/ijp/3/1/5

77. Т. А. Агекян. Звёзды, галактики, Метагалактика. - М.: Наука, Главная редакция физико-математической литературы, 1981.

78. Ф. Ф. Менде Ферроэлектрический трансформатор. Инженерная физика, №4, 2012, c. 15-16.

URL: http://infiz.tgizd.ru/ru/arhiv/9799 


\section{отзыв}

на монографию Менде Ф. Ф. Дубровина А. С. «Альтернативная идеология электродинамики», 262 с., ил. 64, библ. 79.

Авторы излагают альтернативную электродинамику, в которой вместо общепринятых на сегодняшний день понятий тензора комплексной диэлектрической проницаемости (тензор магнитной проницаемости для анизотропных сред (в том числе ограниченных) неоднозначно определен и не нужен), учитывающего частотную (временную) и пространственную дисперсию, предлагается использовать кинематические индуктивность и емкость. При этом они как бы ограничиваются изотропными средами, говоря о магнитной и диэлектрической проницаемостях. Авторы для полноты изложения вводят понятие векторного потенциала электрического поля и уточняют роль векторного потенциала магнитного поля в уравнениях индукции. Показано, что кинетическая индуктивность зарядов играет в электродинамике не менее важную роль, чем диэлектрическая и магнитная проницаемость. Разработана математическая модель временной дисперсии электромагнитных волн в проводниках и диэлектриках с использованием физической величины диэлектрической проницаемости, которая не зависит от частоты. Показано, что в ограниченной плазме может существовать поперечный плазменный резонанс. Вводится понятие кинетической ёмкости. Вводится скалярно-векторный потенциал, в котором скалярный потенциал заряда и его поля зависят от скорости. Из симметричных законов индукции в рамках преобразований Галилея получены преобразования полей при переходе из одной инерционной системы отсчёта в другую. Эти преобразования позволили объяснить фазовую аберрацию и поперечный эффект Доплера, а также силовое взаимодействие токонесущих систем без использования постулата о силе Лоренца. Приведены результаты экспериментальных исследований, показавших, что заряд не является инвариантом скорости.

Изложенный авторами подход удобен для ограниченных в пространстве систем, с размерами, много больше размеров неоднородности поля (длины волны излечения). Они заимствованы из электротехники, и авторы сравнивают свои результаты с экспериментом из электромагнитостатики. Эффекты пространственной дисперсии в этой области несущественны, и авторы законно ими пренебрегают.

Книга предназначена для специалистов в области электротехники и электроники, радиофизики и радиотехники, технической защиты информации. Её можно рекомендовать также студентам и аспирантам, специализирующимся в указанных областях науки.

Я с чистой совестью рекомендую книгу для публикации, хотя с некоторыми критическими замечаниями не согласен. В связи с этим хотел бы авторам дать песколько дружеских замечаний, которые они, если захотят, могут учесть. 
1. По-моему, авторы приписывают СТО большее, чем то, на что она претендует. Именно преобразования Лореца в изложении Пуанкаре относятся к теории поля, т.е. к линейной электродинамике пространственно неограниченной пустоты (я даже не упоминаю слово «вакуумх). Сами уравнения Максвелла, дополненные уравнениями движения среды, могут являться Лоренц не инвариантными, хотя правильно описывають исследуемый процесс.

2. Наконец, тензор диэлектрической проницаемости, учитывающий пространственную дисперсию, строго говоря, можно вводить для неограниченных в пространстве систем (размеры которых много больше размера неоднородности полей). Это не замечание, а пожелание авторам, если они будут развивать дальше свои представления и будут учитывать пространственную дисперсию.

Лауреат государственных премий и премии

им. М.В. Ломоносова, профессор

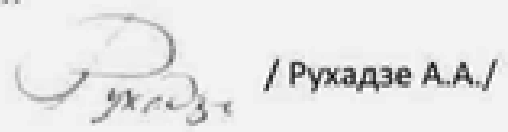

$$
12.0 \% 15
$$

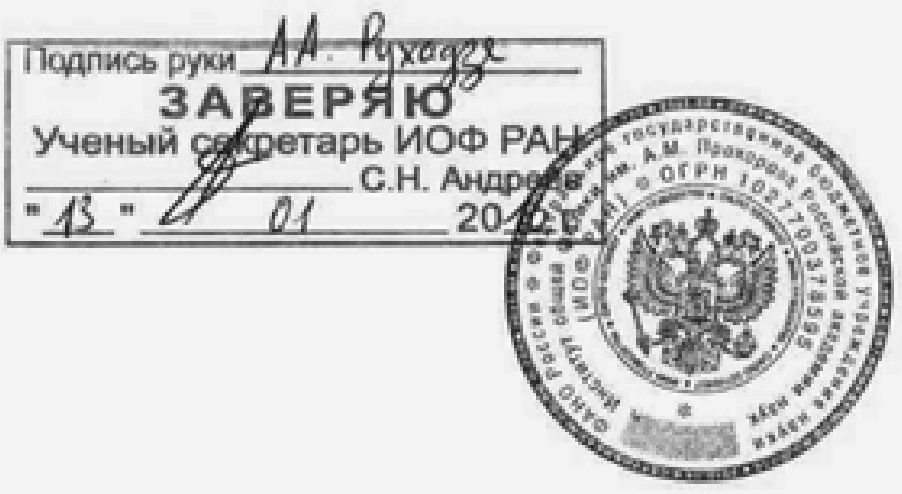




\section{Отзыв}

на монографию Менде Ф. Ф. Дубровина А. С. «Альтернативная идеология электродинамики» 262 с., ил. 64, библ. 79.

Современная классическая электродинамика состоит из двух не связанных между собой частей. С одной стороны это уравнения Максвелла, описывающие волновые процессы, с другой стороны - закон Ампера, описывающий силовое взаимодействие токонесущих систем, и до настоящего времени не было того звена, которое могло объединить эти разрозненные части.

Впервые законы индукции, из которых следуют уравнения Максвелла, были записаны в его знаменитом трактате, где было введено понятие тока смещения и векторный потенциал магнитного поля. Но не все физики знают, что те уравнения, которые принято называть именем Максвелла, это те уравнения, которые записаны в его знаменитом трактате. Свои уравнения Максвелл записывал в кв์Трнионной форме с использование субстанциональной производной. В дальнейшем Герц и Хевисайд исключили из субстанциональной производной её конвективную часть, записав уравнения индукции в частных производных. Недостатком такого подхода является невозможность получения преобразования полей при переходе из одной инерциальной системы в другую. Следует, однако, отметить, что Максвеллом был использован только один закон индукции, который принято называть законом индукции Фарадея, и который указывает на то, каким образом изменение магнитных полей приводит к появлению полей электрических. Однако симметричный закон индукции, который показывает, каким образом изменение электрических полей приводит к появлению полей магнитных, Максвелл так и не записал. Однако, если следовать Максвеллу, и записать эти оба уравнения индукции с использованием субстанциональной производной, то можно симметризировать эти законы. Из такой симметризации следует зависимость скалярного потенциала заряда от скорости. Такой подход даёт возможность ввести понятие скалярно-векторного потенциала, а также законы преобразования полей при переходе из одной инерциальной системы в другую в рамках преобразований Галилей. Эти результат следует считать наиболее важным в рецензируемой монографии. Дальнейшее развитие этих идей дало возможность получить указанные преобразования матричным способом, а также в рамках транскоординатной электродинамики, основные идеи которой также представлены в монографии. Более того, такой подход позволил связать воедино разрозненные части электродинамики и показал, что зависимость скалярного потенциала от скорости является основой всех законов электродинамики, связанных с движением зарядов. Симметризация законов индукции позволила также ввести такое новое понятие, как кинетическая ёмкость,

В монографии уточняется роль векторного потенциала магнитного поля. в уравнениях индукции и вводится понятие векторного потенциала электрического поля. Показано, что кинетическая индуктивность зарядов играет в электродинамике не менее важную роль, чем диэлектрическая и магнитная проницаемость. Разработана математическая модель дисперсии электромагнитных волн в проводниках и диэлектриках с использованием физической величины диэлектрической проницаемости, которая от частоты не зависит. Показано, что в ограниченной плазме может существовать поперечный плазменный резонанс. Приведен новый способ вывода волнового уравнения. Приведены результаты экспериментальных исследований, показавших, что заряд не является инвариантом скорости. Монография заслуживает высоко оценки и может быть рекомендована для публикации.

Заведующий кафедрой прикладной элячро инамики Вадиофизического факультета

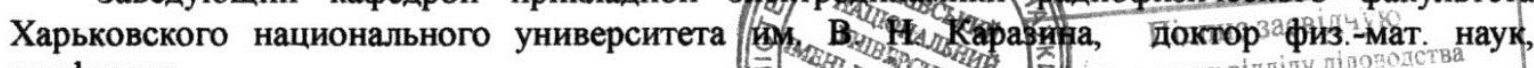
профессор

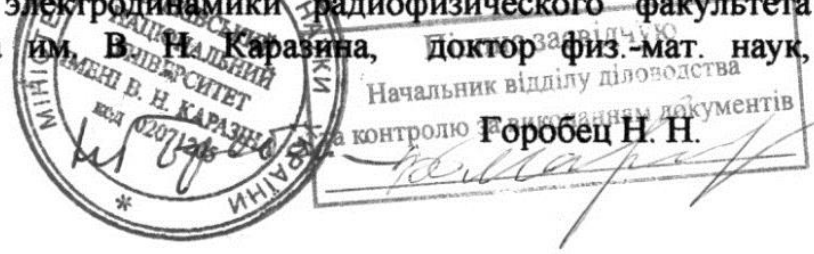




\section{ОГЛАВЛЕНИЕ}

ВВЕДЕНИЕ

ЧАСТЬ I

УТОЧНЕНИЕ ПОНЯТИЙ КЛАССИЧЕСКОЙ

ЭЛЕКТРОДИНАМИКИ

ГЛАВА 1

ЗАКОНЫ ИНДУКЦИИ И ИХ РОЛЬ В КЛАССИЧЕСКОЙ

ЭЛЕКТРОДИНАМИКЕ

$\S 1$. Уравнения Максвелла и сила Лоренца ……..................................... 10

$\S 2$. Закон магнитоэлектрической индукции .......................................... 13

$\S 3$. Закон электромагнитной индукции ............................................. 21

$\S 4$. Множественность форм записи законов электродинамики ........... 23

ГЛАВА 2

РОЛЬ И МЕСТО КИНЕТИЧЕКОЙ ИНДУКТИВНОСТИ

ЗАРЯДОВ В СОВРЕМЕННОЙ ЭЛЕКТРОДИНАМИКЕ

$\S 5$. Кто и как ввёл частотную дисперсию диэлектрической

проницаемости материальных сред ……………............................ 25

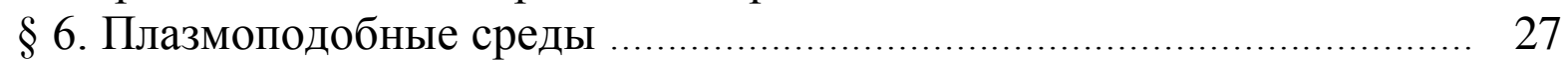

§ 7. Поперечный плазменный резонанс .............................................. 36

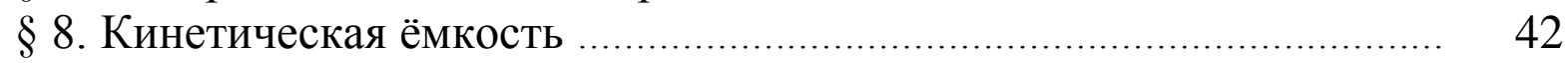

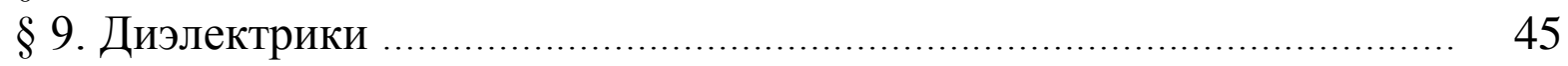

ГЛАВА 3

НОВЫЕ МЕТОДИКИ И ПОНЯТИЯ ……….................................. 53

$\S 10$. Поверхностная кинетическая индуктивность ............................... 53

$\S$ 11. Электроёмкостная самоиндукция ……....................................... 54

§ 12. Индуктивнотоковая самоиндукция ............................................. 57

$\S 13$. Новый способ получения волнового уравнения, потенциальные

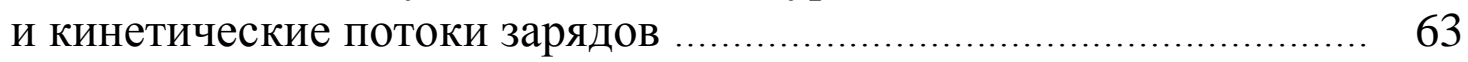

§ 14. Переходные процессы в отрезках длинных линий ....................... 70

ЧАСТЬ II

НОВЫЕ ИДЕИ В КЛАССИЧЕСКОЙ ЭЛЕКТРОДИНАМИКЕ ..... 76

ГЛАВА 4

НОВЫЕ ПОДХОДЫ И ОПРЕДЕЛЕНИЯ .................................... 76

$\S 15$. Динамические потенциалы и поля движущихся зарядов .............. 76 
$\S 16$. Уравнения Власова и лагранжиан движущегося заряда в концепции скалярно-векторного потенциала ............................. 84

$\S 17$. Транскоординатная электродинамика в пространственновременном гиперконтинууме и преобразования Менде

$\S 18$. Электрические поля в концепции скалярно-векторного потенциала

$\S 19$. Силовое взаимодействие токонесущих систем, униполярная индукция и пондеромоторные силы

$\S 20$. Экспериментальное подтверждение зависимости скалярного потенциала заряда от его относительной скорости

$\S 21$. Электрический импульс ядерного взрыва

$\S 22$. Теоретические основы гиперконтинуальной физики и гиперконтинуальной электродинамики

$\S 23$. Особенности математического аппарата классической электродинамики

ЧАСТЬ III

ДРУГИЕ НОВЫЕ ГИПОТЕЗЫ, ЭКСПЕРИМЕНТАЛЬНЫЕ

РЕЗУЛЬТАТЫ И ТЕХНИЧЕСКИЕ РЕШЕНИЯ

ГЛАВА 5

ДРУГИЕ ГИПОТЕЗЫ, ЭКСПЕРИМЕНТАЛЬНЫЕ

РЕЗУЛЬТАТЫ И ТЕХНИЧЕ СКИЕ РЕШЕНИЯ

$\S 25$. Являются ли лазер и квантовый генератор квантовыми генераторами ...

$\S 26$. Физическое обоснование принципа Гюйгенса и теорема взаимности направленных антенн .

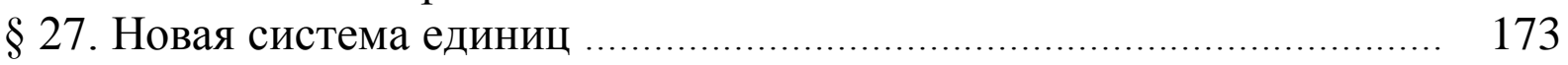

$\S 28$. Капельная модель электрона и атома ....................................... 175

§ 29. Гравитационный дефект масс .................................................... 179

$\S 30$. Пространственная когерентизация ………................................... 180

§ 31. Ферроэлектрический трансформатор ………............................... 181

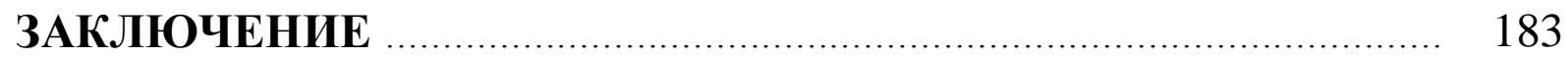

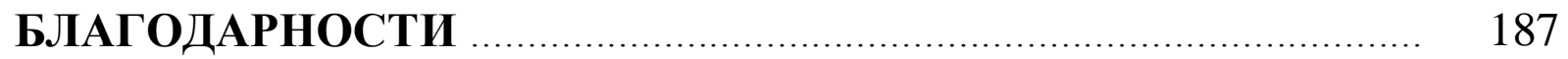

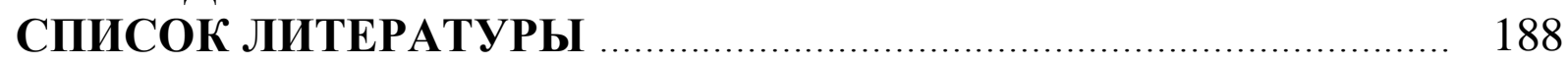

Отзыв профессора А. А. Рухадзе ................................................ 193

Отзыв профессора Н. Н. Горобца ……..................................... 195 
УДК 537.812+537.312.62+621.372.834

Менде Ф. Ф., Дубровин А. С.

Альтернативная идеология электродинамики. М.: Перо, 2016. - 198 с., ил. 62, библ. 78.

ББК 22.313

Менде Фёдор Фёдорович, Дубровин Анатолий Станиславович mende_fedor@mail.ru asd_kiziltash@mail.ru

АЛЬТЕРНАТИВНАЯ ИДЕОЛОГИЯ ЭЛЕКТРОДИНАМИКИ 